\title{
Effects of symmetry breaking in low dimensional materials
}

Agosto de 2021

Autor: Mário Jorge César dos Santos

Director: D. Francisco Javier Manjón Herrera 

"The fundamental laws necessary for the mathematical treatment of a large part of physics and the whole of chemistry are thus completely known, and the difficulty lies only in the fact that application of these laws leads to equations that are too complex to be solved."

Paul Dirac 



\section{Acknowledgments}

First of all I want to thank Professor Francisco Javier Manjón, my thesis supervisor, for giving me the opportunity to work with him and the great effort he has put into getting my thesis move along.

I am grateful to Prof.Yuriy Pogorelov, who from the initial stages, dedicated his time to teach me. Throughout this work, his constant encouragement and support have kept my enthusiasm alive. I also owe him all the suggestions that made it possible to improve this work, since the preliminary version, a very brief draft with several inaccuracies, until the final result, there was a long and rich learning path.

The work of this thesis was carried out in collaboration with several researchers at the Universities of Coimbra and Aveiro (Portugal) of whom I also want to thank their dedication. I recall the ideas of some discussions with these research groups, in which I had the pleasure of participating, and who helped me achieve this work.

Many other friends have also supported me enormously during the thesis and I have cheered at all times, in particular my very good friend Prof. Tao Yang (Shenzhen Technology University).

Finally I want to thank my family, who will always be a reference for me, and the support that they have always given me in everything I have done, not only throughout writing this thesis but also to my life. 



\section{Abstract}

The dimensionality of the system plays a decisive role in the behavior of the electronic dynamics of interacting electrons. In particular, the quasi-2D dimensionality is responsible for the unusual behavior observed in graphene-like materials and layered van-der-Waal systems. Moreover, such effects are also observed for superconducting materials of high critical temperature, even in the normal state, due to their low-dimensionality.

The experimental study of graphene triggered a growing attention to respective electronic properties, because the honeycomb lattice defines a band structure with two nodal points in the Brillouin zone which determines a relativistic Dirac-type electronic dynamics. Within a theoretical framework, many properties of single-layer graphene have been studied to allow further characterization of this material. These properties are unconventional due to the unique band structure of graphene, which is described in terms of Dirac fermions, creating links with certain theories of particle physics. In fact, several theoretical groups have employed phenomenological models inspired in quantum cromodynamics (i.e. Nambu-Jona Lassino and Gross-Neveu models) applied to the study of graphene properties. These properties are responsible for the unusual phenomena, such as the fractional Hall effect, which allows the possibility for magnetic catalysis of an excitonic gap, ferromagnetism and superconductivity.

The research of high critical temperature superconductors with impurity centers is significant for understanding the underlying physics of such disordered systems. While the cuprate family present insulating properties in the pristine state, the undoped iron pnictides (i.e. LaOFeAs) show a semi-metallic behav- 
ior. Inspite these diferences, both compounds are layered structures, where the superconducting state is supported by a quasi-2D square lattice. While for iron pnictides this state is formed by the FeAs layer, the cuprate superconducting state is formed by the $\mathrm{CuO}$ layer.

The current work focuses on the theoretical study of the structural, electronic and optical properties of graphene-type materials, such as bilayer graphene; and also of s- and d-wave superconductors, more specifically iron pnictides and cuprates, respectively. Furthermore, disordered systems will be focused upon since these (quasi-)2D systems are quite sensitive to disorder. Such properties have major importance for technological device applications, as can be observed in the increasing technological fields of high temperature superconductores and electronic devices. The type of perturbations applied to the systems of interest are chemical impurities and/or external electric bias, and these show variations of the electronic and optical properties when compared to the pristine systems.

The electronic properties are described by calculating the spectrum of the material by employing the Tight-Binding theory and the continuum model. In the case of the graphene system, by performing the calculation in the low energy limit we obtain the linear dispersion, which is one of the characteristics of the Dirac massless relativistic particles. Other studies related to suspended graphene in vacuum, revealed that graphene can change from a semi-metal into an insulator, due to the formation of a gap in the fermionic spectrum, resultant from the chiral condensate (exciton). The Dirac equation allows to further probe the strain effects, leading to deformed graphene, which can be seen as a tabletop for quantum field theories in curved spacetimes.

Green function methods is the most robust and common analytical tool to compute the physical observables, such as the density of states (DoS). The DoS is a fundamental physical quantity which aids in the interpretation of several experimental data mostly for disordered systems.

The effect of impurities is calculated by employing the simple and self-consistent T-matrix. For the considered case of finite impurity concentration, the selfconsistent method is more reliable. However, with the exception of the 'spurious' effect obtained for the vacancy (DoS divergence), that persists even with the self-consistent technique, all other observables treated within this formalism will be in accordance with the experimental data and other theoretical approximations.

Other techniques employed to study the structural and electronic properties of these systems of interest are based on computational methods, such as Density 
Functional Theory and these serve as a complementary tool to the analytical methods. Methodologies based on phonon theory are also employed to probe the dynamical stability of the systems. 



\section{Resumen}

La dimensionalidad de un sistema juega un papel fundamental en la conducta de la dinámica de los electrones que interactúan. En particular, la dimensionalidad cuasi-2D es responsable del comportamiento inusual observado en materiales de tipo grafeno y sistemas laminares basados en enlaces de tipo van der Waals. Además, estos efectos también se observan en materiales superconductores de alta temperatura crítica, incluso en el estado normal, debido a su baja dimensionalidad.

El estudio experimental del grafeno provocó una atención creciente a sus propiedades electrónicas, porque su estructura en forma de panal de abejas da lugar a una estructura de bandas con dos puntos nodales en la zona de Brillouin que determina una dinámica electrónica relativista de tipo Dirac. En el plano teórico, muchas propiedades del grafeno de una sola capa se han estudiado para permitir una mayor caracterización de este material. Estas propiedades son poco convencionales debido a la singular estructura de bandas del grafeno, que se describe en términos de fermiones de Dirac, lo que crea vínculos con ciertas teorías de la física de partículas. De hecho, varios grupos teóricos han empleado modelos fenomenológicos inspirados en la cromodinámica cuántica (es decir, los modelos Nambu-Jona Lassino y Gross-Neveu) aplicados al estudio de las propiedades del grafeno. Estas propiedades son responsables de inusuales fenómenos, como el efecto Hall fraccionario, que permite la posibilidad de catálisis magnética de un gap excitónico, ferromagnetismo y superconductividad.

La investigación de superconductores de alta temperatura crítica con centros de impurezas es importante para comprender la física subyacente de tales sistemas 
desordenados. Mientras que la familia de los cupratos presenta propiedades aislantes en estado prístino, los pnictogenuros de hierro sin dopar (es decir, LaOFeAs) muestran un comportamiento semimetálico. A pesar de estas diferencias, ambos compuestos son estructuras en capas, donde el estado superconductor está respaldado por una red cuadrada cuasi-2D. Mientras que para los pnictogenuros de hierro este estado está formado por la capa de FeAs, el estado superconductor de cuprato está formado por la capa de $\mathrm{CuO}$.

El presente trabajo se centra en el estudio teórico de las propiedades estructurales, electrónicas y ópticas de los materiales de tipo grafeno, como el grafeno bicapa; y también de superconductores de ondas s y d, más específicamente pnictogenuros y cupratos de hierro, respectivamente. Además, se hace hincapié en sistemas desordenados ya que estos sistemas (cuasi-)2D son bastante sensibles al desorden. Tales propiedades tienen gran importancia para aplicaciones de dispositivos tecnológicos, como se puede observar en la creciente tecnología campos de tensiotrónica y espintrónica. El tipo de perturbaciones aplicadas a los sistemas de interés son las impurezas químicas y campos eléctricos externos. Estas perturbaciones producen variaciones de las propiedades electrónicas y ópticas cuando se comparan con los sistemas prístinos.

Las propiedades electrónicas se describen calculando el espectro del material mediante la teoría Tight-Binding y el modelo continuo. En el caso del sistema de grafeno, al realizar el cálculo en el límite de baja energía se obtiene la dispersión lineal que es una de las características de las partículas relativistas sin masa de Dirac. Otros estudios del grafeno suspendido en el vacío revelaron que el grafeno puede cambiar de semi-metal a aislante debido a la formación de un espacio en el espectro fermiónico de electrones que resulta del condensado quiral (excitón). La ecuación de Dirac permite sondear aún más los efectos de la tensión, lo que lleva a un grafeno deformado, que puede verse como un campo de pruebas para las teorías cuánticas de campos en espacio-tiempos curvos.

Los métodos basados en las funciones de Green son las herramientas analíticas más robustas y comunes para calcular los observables físicos, como la densidad de estados (DoS). La DoS es una magnitud física fundamental que ayuda en la interpretación de varios datos experimentales principalmente para sistemas desordenados. El efecto de las impurezas se calcula empleando el método simple y autoconsistente Matriz en T. Para el caso considerado de concentración finita de impurezas, el método autoconsistente es más fiable. Sin embargo, con la excepción del efecto "espurio" obtenido para la vacante (divergencia DoS), que persiste incluso con la técnica autoconsistente, todos los demás ob- 
servables tratados dentro de este formalismo estarán de acuerdo con los datos experimentales y otras aproximaciones teóricas.

Otras técnicas empleadas para estudiar las propiedades estructurales y electrónicas de estos interesantes sistemas se basan en métodos computacionales, como la teoría del funcional de la densidad, que sirven como herramientas complementarias a los métodos analíticos. Finalmente, cabe mencionar que también se emplean metodologías basadas en la teoría de fonones para investigar la estabilidad dinámica y la conductividad térmica de la red. 



\section{Resum}

La dimensionalitat d'un sistema juga un paper fonamental en la conducta de la dinámica dels electrons que interactúen. En particular, la dimensionalitat cuasi-2D és responsable del comportament inusual observat a materials de tipus grafè i sistemes laminars basats en enllaços de tipus van der Waals. A més a més, aquestos efectes també s'observen a materials superconductors d'alta temperatura crítica, inclús al seu estat normal, degut a la seua baixa dimensionalitat.

L'estudi experimental del grafè va produir una atenció creixent a les seues propietats electròniques, perque la seua estructura en forma de panal d'abelles dona lloc a una estructura de bandes amb dos punts nodals a la zona de Brillouin que determinen una dinámica electrónica relativista de tipus Dirac. Al planol teòric, moltes propietats del grafè d'una sola capa s'han estudiat per a permetre una major caracterizació d'aquest material. Aquestes propietat són poc convencionals degut a la singular estructura de bandes del grafè, que es descriu mitjançant fermions de Dirac. Aquestos fermions permeten establir víncles amb certes teories de la física de particles. De fet, alguns grups teòrics han empleat models fenomenològics inspirats a la cromodinàmica quàntica (es a dir, els models Nambu-Jona Lassino i Gross-Neveu) aplicats a l'estudi de les propietats del grafè. Aquestes propietats són responsables d'inusuals fenómens, com l'efecte Hall fraccionari, que permet la possibilitat de catálisi magnètica d'un gap excitònic, ferromagnetisme i superconductivitat.

La investigació de superconductors d'alta temperatura crítica amb centres d'impureses és important per a comprendre la física subjacent de tals sistemes 
desordenats. Mentre que la família dels cuprats presenta propietats aïllants en estat pristí, els pnictogenurs de ferro sense dopar (és a dir, LaOFeAs) mostren un comportament semimetálico. Malgrat aquestes diferències, tots dos compostos són estructures en capes, on l'estat superconductor està recolzat per una xarxa quadrada quasi-2D. Mentre que per als pnictogenurs de ferro aquest estat està format per la capa de FeAs, l'estat superconductor dels cuprats està format per la capa de $\mathrm{CuO}$.

El present treball es centra en l'estudi teòric de les propietats estructurals, electròniques i òptiques dels materials de tipus grafè, com el grafè bicapa; i també de superconductors d'ones s i d, més específicament pnictogenurs i cuprats de ferro, respectivament. A més a més, es fa emfasi en sistemes desordenats ja que aquestos sistemes (cuasi-)2D són prou sensibles al desordre. Aquestes propietats tenen gran importància per a aplicacions de dispositius tecnològics, com es pot observar a la creixent tecnologia dels camps de la tensiotrònica i l'espintrònica. El tipus de pertorbacions aplicades als sistemes d'interés són les impureses químiques i els camps elèctrics externs. Aquestes pertorbacions produeixen variacions de les propietats electròniques i òptiques quan es comparen amb els sistemes pristins.

Les propietats electròniques es descriuen calculant l'espectre del material mitjançant la teoría Tight-Binding i el model continu. En el cas del sistema de grafé, al realitzar aquest càlcul al límit de baixa energia s' obté la dispersió lineal que és una de les característiques de les particles relativistes sense massa de Dirac. Altres estudis del grafè suspès al buit van revelar que el grafè pot canviar de semi-metal a aïllant degut a la formació d'un espai a l'espectre fermiònic d'electrons que resulta del condensat quiral (excitó). L'ecuació de Dirac permet sondejar encara més els efectes de la tensió, el que condueix al grafè deformat, que pot considerar-se com un tauler per a provar les teories quàntiques de camps als espais-temps curvos.

Els mètodes basats a les funcions de Green són les ferramentes analítiques més robustes i comuns per a calcular els observables físics, com la densitat d'estats (DoS). La DoS és una magnitut física fonamental que ajuda a l'interpretació de diverses dades experimentals principalment per a sistemes desordenats. L'efecte de les impureses es calcula empleant el mètode simple i autoconsistent anomenat Matriu en T. Per al cas considerat de concentració finita d'impureses, el mètode autoconsistent és més fiable. Tanmateix, amb l'excepció de l'efecte "espurio" obtés per a la vacant (divergència DoS), que persisteix inclús amb la tècnica autoconsistent, tots els altres observables tractats dins d'aquest formalisme estaran d' acord amb les dades experimentals i altres aproximacions teòriques. 
Altres tècniques emprades per a estudiar les propietats estructurals i electròniques d'aquestos interesants sistemes es basen a mètodes computacionals, com la teoria del funcional de la densitat, que serveixen com a ferramentes complementàries als mètodes analítics. Finalment, cal esmentar que també s'empren metodologies basades a la teoria de fonons per a investigar l'estabilitat dinàmica i la conductividad tèrmica de la xarxa. 



\section{Contents}

Abstract

Contents $x i x$

1 Introduction and Thesis Objectives 1

1.1 High Critical Temperature Superconductivity . . . . . . . . . . . . . . 1

1.2 Graphene-Based Systems . . . . . . . . . . . . . . . . . . . . 3

1.3 Chapter Structure of the Thesis . . . . . . . . . . . . . . . . . . 4

$\begin{array}{lll}2 & \text { Theoretical Framework } & 7\end{array}$

2.1 Crystalline Order and Disordered States . . . . . . . . . . . . . . . . 7

2.2 Tight-Binding Approximation . . . . . . . . . . . . . . . . . . . 22

2.3 Green Function's Methods for Description of Disorder Materials . . . . . . 25

2.4 Density Functional Theory . . . . . . . . . . . . . . . . . . . . . . . 29

2.5 High Critical Temperature Superconductors . . . . . . . . . . . . . . . 40

2.6 Overview of Low-Dimensional Physics in 2D Graphene . . . . . . . . . . 50 
3 Effects of extended impurity perturbation in d-wave superconductor

4 Specifics of impurity effects in ferropnictide superconductors 87

5 Impurity effects on electronic transport in ferropnictide superconductors

6 Electric bias control on impurity effects in bigraphene

7 Electronic and Phonon Instabilities in Bilayer Graphene under Applied External Bias

8 Conclusions and General Discussion of the Thesis Results 175
8.1 High Critical Temperature Superconductivity . . . . . . . . . . . . . . 175
8.2 Graphene-Based Systems . . . . . . . . . . . . . . . . . . . . . 177
8.3 Future Perspectives . . . . . . . . . . . . . . . . . . . . . 178 


\section{Introduction and Thesis Objectives}

\subsection{High Critical Temperature Superconductivity}

During a long period, since the discovery of the superconductivity phenomenon in 1911 by Kammerlingh Onnes, in mercury cooled by liquid helium [1], this phenomenon had solely been observed in pure metals (lead, niobium, iridium). Research persisted on the search for materials which could exhibit transition temperatures for the superconducting (SC) state above $10 \mathrm{~K}$. This was achieved $[2,3]$, for discovered superconductivity on the compound, $\mathrm{V}_{3} \mathrm{Si}$, revealing $\mathrm{T}_{c} \approx$ $17 \mathrm{~K}$.

Conventional materials exhibiting superconducting transition, required an extremely expensive cooling system in order to induce the SC state. The production of artificial materials with SC transition temperatures within the range of liquid nitrogen $(77.4 \mathrm{~K})$, offered new possibilities for the superconductivity phenomenon.

In 1986, two physicists [4], revealed the existence of the SC state at temperatures higher than $30 \mathrm{~K}$ on a ceramic. Immediately after, yttrium-based ceramics were synthesized [5] which pass into the SC state at critical temperatures 
$\left(\mathrm{T}_{c}\right)$ close to $90 \mathrm{~K}$. Since then, several superconductors with $\mathrm{T}_{c}>30 \mathrm{~K}$ have been synthesized, and known as the high critical temperature superconductors (HTSC) [6]. Before 2008, the term of HTSC referred to the copper-based superconductors (discovered in 1986), also known as cuprates. Currently this term is also used to designate the iron-based superconductors, which reveal a complex phase diagram where magnetism and superconductivity interact. This fact is also typical of other exotic superconductors where it is well known that the coexistence of magnetism and superconductivity is unusual.

The phenomenon of superconductivity of high critical temperature in cuprates is directly related to the $\mathrm{CuO}_{2}$ planes, while atoms of the neighboring layers provide these with charge carriers (electrons or holes), thus leading to the metallization and to superconductivity. This is the reason why the neighboring layers of $\mathrm{CuO}_{2}$ planes are called the "reservoirs of charge". The assumption that superconductivity of high critical temperature is a phenomenon in two dimensions, is based on the fact that the distance between two $\mathrm{CuO}_{2}$ planes is greater than the distance between the copper and oxygen atoms in those planes, such that electrons (or holes) are most likely shared by these atoms in the planes, than outside of them.

Doping is the mechanism that enables the charge transfer, of the "charge reservoirs" to the SC planes. Namely, this is the substitution of atoms, of the "charge reservoirs", by other atoms with different ionization state, such that electrons are allowed to pass between $\mathrm{CuO}_{2}$ planes, or are withdrawn thereof. Typically, the HTSCs with hole doping have a higher critical temperature than those with electron doping.

One of the objectives of this thesis is to study the effects of disorder, mainly by impurities, on the electronic properties of doped iron pnictides and cupratebased HTSCs. The results are presented in chapters three, four and five. Impurity centers that are essential to form the SC state, result either from dopants, or from foreign atoms and other local defects. Within the minimal coupling model, it will be shown that the localized impurity levels (even for the simplest isotopic impurity perturbations) form within the SC gaps in doped iron pnictides, e.g LaOFeAs. The results which will be presented in the present thesis can serve for two purposes:

- Estimate the possibility that impurity excitations at high enough impurity concentrations can form coherent collective states, known as the impurity bands, or these always remain localized states, and 
- Describe more detailed characteristics of each type of impurity states, the dispersion law and the lifetime for band-like states, or the density of localized states, and the positions of the boundaries between the Mott mobility edges.

\subsection{Graphene-Based Systems}

Many properties of single-layer graphene have been theoretically studied to allow further characterization of this material. These properties are unconventional due to the unique band structure of graphene, which is described in terms of Dirac fermions.

The experimental study of graphene triggered a growing attention to its electronic properties [7], because the honeycomb lattice defines a band structure [8] with two nodal points in the Brillouin zone (BZ) which determines a relativistic Dirac-type electronic dynamics [9] (creating links with certain theories of particle physics). These properties are responsible for unusual phenomena, such as the fractional Hall effect [10, 11, 12, 13], which allows the possibility for magnetic catalysis of an excitonic gap [14, 15, 16, 17, 18], ferromagnetism and superconductivity [19].

Other studies related to suspended graphene in vacuum, revealed that graphene can change from a semi-metal into an insulator, due to the formation of a gap in the fermionic spectrum, resultant from the chiral condensate (exciton) [11, 20].

More recently, attention has turned to the multi-layer graphene [21] and, particularly, to the bilayer graphene, which also reveals interesting aspects, e.g., on the of Quantum Hall effect [10]. In fact, it was shown that the bilayer graphene also shows unconventional behavior in its properties, however, these properties are different from those observed a single-layer of graphene.

There are two main reasons that explain the unconventional physics of multilayered graphene:

- The coupling between the layers is relatively weak and therefore some of the properties of the base material, the single layer of graphene, are manifested.

- The peculiar geometry resulting from the A-B layer stacking (Bernal stacking), implies that the connection between the two planes takes place mostly in one of the sub-lattices of each plane. 
As in the case of monolayer graphene, also the bilayer graphene is sensitive to the inevitable disorder.

It has been theoretically and experimentally shown that the bilayer graphene is a material with semiconductor properties in which the width of the electronic gap is proportional to an applied electric field.

Another objective of the present thesis is the study of the effect of perturbation, by considering an applied electric field normal to the stacking direction, in bilayer graphene. The results on this subject will be presented in chapters six and seven. For that purpose, we will start by describing the pure bilayer graphene system with Bernal stacking, where we only consider the $t_{z}$ coupling amplitude between vertical layers restricted to shorter vertical distances of these carbon atoms. We will apply the electric field, $E$, between these planes, by considering a potential, $V=e E d$, between the layers (where $e$ is the electron charge and $d$ the interlayer spacing).

\subsection{Chapter Structure of the Thesis}

Chapter two contains a section where some concepts applied to solid crystalline systems, namely crystalline order and description of disordered and surface states will be introduced. It follows with three sections with a detailed description of the theoretical framework employed to perform the calculations presented in this thesis, and will be used to pursue the objectives mentioned in sections 1.1 and 1.2. The last two sections will provide an overview of the description and properties of high critical temperature superconductors and graphene systems.

Chapter three, which refers to the first article of this thesis is based on the study of "Effects of extended impurity perturbation in $d$-wave superconductor" where we present with some detail the results obtained from work related to the study of $d$-wave superconductors (Impurity clusters and localization of nodal quasiparticles in d-wave superconductors) [22] and extended s-wave superconductors (Specifics of impurity effects in ferropnictide superconductors) [23]. Although the intrinsic differences that exist between the iron and copper superconductors, the phase diagrams and methodology for the study of the physics of the superconductivity are very similar.

The second article (chapter four), entitled "Specifics of impurity effects in ferropnictide superconductors" specifies the study of the effect that impurities produce in the spectra of iron-pnictides, where localized energy states may 
occur inside the superconductor gap. We also study the modification of the band-structure and the superconducting order-parameter, as well as the evolution of the superconducting state related to the impurity doping.

The third article (chapter five) is based on the study of "Impurity effects on electronic transport in ferropnictide superconductors" where we describe the effects that impurities and disorder by electronic quasiparticles, can cause on the transport properties of superconducting iron pnictides. We show that the most prominent features appear at high enough impurity concentration, when compared to the case of a pristine system. These features consist in the formation of localized in-gap impurity states and their development into specific narrow bands of impurity quasiparticles when the impurity concentration goes above a certain (quite low) critical value. The predicted specific threshold effects in the frequency dependent optical conductivity and temperature dependent thermal conductivity and also in Seebeck and Peltier coefficients can have interesting potentialities for practical applications.

The fourth publication of chapter six, is related to "Electric bias control on impurity effects in bigraphene". In this work, we study the localized impurity levels on the $\mathrm{AB}$ bilayer graphene system. It is shown that a qualitative restructuring of quasiparticle spectrum within the initial band gap and then specific metal-insulator phase transitions are possible for such a disordered system at a given impurity perturbation potential and concentration, and for which such processes can be effectively controlled by variation of an external electric field bias.

Chapter seven, with the fifth article, refers to "Electronic and Phonon Instabilities in Bilayer Graphene under Applied External Bias". This article introduces the application of Density Functional Theory (DFT) and lattice dynamics to probe the dynamical stabilities of the $\mathrm{AB}$ and $\mathrm{AA}$ bilayer stacking, and the effect of applying an external bias to the electronic and phonon properties.

The last chapter refers to the conclusions and general discussion of the thesis results, and includes a section with some perspectives of continuation of the work presented in the present thesis. 



\section{Theoretical Framework}

\subsection{Crystalline Order and Disordered States}

Crystalline order, or lattice periodicity, is the most rigorous form of order, and it is the simplest arrangement of atoms or molecules in a repeating pattern, forming a macroscopic solid. The full information of the crystal can be contained in a small region in space, determined by the unit-cell parameters. The pattern is periodically repeated to set up a translationally invariant space $[24,25,26,27]$.

Auguste Bravais showed that in three-dimensional (3D) crystal, there existed 14 different possibilities of arranging different group of points in space - named as the Bravais lattices. The simplest type of lattice is the Bravais lattice, where the location of all the points are constructed by basic translation though three primitive lattice vectors $\mathbf{a}_{1}, \mathbf{a}_{2}, \mathbf{a}_{3}$. If the crystal is shifted by any of these three vectors, or a combination of them of the form $n_{1} \mathbf{a}_{1}+n_{2} \mathbf{a}_{2}+n_{3} \mathbf{a}_{3}$, where $n_{i}$ are three integers, then the atoms end up in the same set of locations as they started, such that, the neighborhoods of all points must be identical under translation.

In a Bravais lattice, the primitive-cell should contain only one point, which is invariant under all symmetry operations [25, 27]. By drawing the perpendicular 
bisector plane of the translation vector from the center point to the nearest equivalent lattice sites, the volume defined inside the bisector planes is the Wigner-Seitz (WS) cell. Moreover, the WS cell of the reciprocal lattice is called first Brillouin Zone (BZ). The unit-cell is the smallest group of points (atoms, ions, molecules) that, when repeated in 3D, will produce the periodic lattice of a bulk crystal system.

The symmetry of a crystal depends on the complete set of ways that a given crystal can be transformed so that the distance between all points are preserved. Rigid motions include translations and rotations, which may also be allowed to reflect and invert. The complete set of rigid motions that take the crystal into itself is called a space group [27]. The space groups in 3D are formed by combinations of the 32 crystallographic point groups with the 14 Bravais lattices, each of these belonging to 7 lattice systems. These 7 different lattice systems are characterized according to the relative lengths of the unit-cell parameters $(a, b, c)$ and the angles between them $(\alpha, \beta, \gamma)$ : triclinic, monoclinic, orthorhombic, tetragonal, rhombohedral, hexagonal, and cubic. The combination of all these symmetry operations results in a total of 230 different space groups describing all possible crystallographic symmetries [28, 29].

The space-group can yet be divided into two sub-groups:

- Translation group, which consists of translations through all lattice vectors (leaving the crystal invariant)

- Point group consists of operations that also leave the crystal invariant and also map some particular Bravais lattice point onto itself.

When two systems share the same point group symmetry, these will belong to the same crystal system, but may be different lattices belonging to different space groups. One may characterize if two structures are the same, by deforming one of the systems continuously into the other structure without lose of symmetry [27]. 


\subsubsection{Electronic States in Crystalline Solids}

\section{Schrödinger Equation of an Electron in a Solid}

Considering a solid formed by $N_{a}$ multi-electron atoms, which contains $N$ electrons, the Schrödinger equation for the crystal can be represented through the following Hamiltonian:

$$
\begin{aligned}
H\left(\ldots, \mathbf{R}_{j}, \ldots, \mathbf{r}_{i}, \ldots\right)= & -\frac{\hbar^{2}}{2} \sum_{j} \frac{1}{M_{j}} \nabla_{j}^{2}-\frac{\hbar^{2}}{2 m_{0}} \sum_{i} \nabla_{i}^{2}+\frac{1}{2} \sum_{j, j^{\prime}}^{\prime} \frac{Z_{j} Z_{j}^{\prime} e^{2}}{\left|\mathbf{R}_{j}-\mathbf{R}_{j}^{\prime}\right|} \\
& +\frac{1}{2} \sum_{i, i^{\prime}}^{\prime} \frac{e^{2}}{\left|\mathbf{r}_{i}-\mathbf{r}_{i}^{\prime}\right|}-\sum_{i, j} \frac{Z_{j} e^{2}}{\left|\mathbf{r}_{i}-\mathbf{R}_{j}\right|}
\end{aligned}
$$

where $\mathbf{R}_{j}$ are the vectors defining the ion position and $\mathbf{r}_{i}$ the electron positions, $M_{j}$ is the mass corresponding to the respective $j$ ion with $Z_{j}$ being the respective atomic number, and $m_{0}$ is the electron mass.

The complete wavefunction will involve all the space coordinates of the nucleus and electrons of the system, being too complex to obtain the exact solution of the Schrödinger equation. Moreover, the electron-ion interactions and the par interactions (ion-ion and electron-electron) do not allow the decomposition of the Schrödinger equation into as many differential equations according to the existing independent parameters. Therefore, approximations are required which will allow the simplification of the interacting many-body problem.

One such approach is the adiabatic approximation $\left(\mathbf{R}_{j}=\right.$ const) [25, 28], (also known as the Born-Oppenheimer approximation) which consists in considering that electrons 'move' in a spacial configuration of nearly constant ('frozen') nucleus motion. The nucleus possess very slow motion (vibrational), allowing the possibility to eliminate several degrees of freedom related to the ion motion.

The mean-field approximation allows reducing the many-body electron problem to a single-particle electron. This is achieved by introducing an effective potential, created by the group of all ions and all electrons, with exception of the considered electron:

$$
v_{e f f}\left(\mathbf{r}_{i}\right)=-\sum_{j} \frac{Z_{j}}{\left|\mathbf{r}_{i}-\mathbf{R}_{j}\right|}+\sum_{i^{\prime} \neq i} e^{2} \int d \mathbf{r}_{i}^{\prime} \frac{\left|\psi_{i}^{\prime}\right|^{2}}{\left|\mathbf{r}_{i}-\mathbf{r}_{i}^{\prime}\right|}
$$


Based on the independent electron picture, the wavefunction of the crystal is the product of the individual electron wavefunctions by:

$$
\Psi\left(\ldots, \mathbf{r}_{i}, \ldots\right)=\Pi_{i=1}^{N} \psi\left(\mathbf{r}_{i}\right) .
$$

The employment of an effective potential and the factorized wavefuntion is known as the Hartree approximation, or as the self-consistent field.

The mean-field theory was later refined to consider the exchange-interaction, or permutation energy, between electrons. In this approach, known as the Hartree-Fock approximation [28], the total wavefunction of each electron is written as the product of the spatial wavefunction (orbital) by the spin wavefunction, and it is anti-symmetrical with respect to all possible permutations of the electron, thus obeying the Pauli exclusion principle. Moreover, the effective potential, $V_{\text {eff }}(\mathbf{r})$, is not explicitly known. The only information about this potential is that it is periodic:

$$
V_{e f f}\left(\mathbf{r}+\mathbf{a}_{n}\right)=V_{e f f}(\mathbf{r})
$$

where $\mathbf{a}_{n}$ is one of the translational vectors of the crystalline lattice. From the periodicity of $V_{e f f}(\mathbf{r})$, it is possible to obtain important information related to the electronic spectrum of the crystal system.

Even after the adoption of the adiabatic approximation, and the mean-field concept, the problem of searching for an adequate effective potential and the approximate solutions to the Schrödinger equation still persists. Even with the current development of the computational resources, it is very difficult to construct a crystalline potential based solely from first-principles (from the electronic structure of the atoms that composes the crystal).

\section{Bloch theorem}

Independent electrons, obeying the Schrödinger equation with a periodic potential are known as the Bloch electrons. Electrons moving in a regular arrangement of atoms forming a crystal are subject to a periodic potential, due to the lattice of ions and an averaged electron-electron interaction. The energy spectrum of the extended electronic states form bands of allowed states (electrons and holes) and gaps of "forbidden" states. The band structure of electrons is what defines the solid into metals, insulators and/or semiconductors. 
There are two limiting cases for the formation of a band structure. One is for the free electron gas, with which the continuous spectrum is perturbed into bands under the influence of a periodic potential (Bragg scattering) [28, 29]. The second limit is due to independent atoms, which are bonded together to form a lattice, until the outermost electronic states overlap, leading to delocalized states. Discrete states are therefore turned into a continua of energy dispersion, forming the bands.

Within the mean-field approximation, the Schrödinger equation for an electron in the crystal can be written as:

$$
-\frac{\hbar}{2 m_{0}} \nabla^{2} \psi(\mathbf{r})+V_{e f f}(\mathbf{r}) \psi(\mathbf{r})=\varepsilon \psi(\mathbf{r}) .
$$

To represent another point in space, distanced from a translational vector, the expression can be re-written as:

$$
-\frac{\hbar}{2 m_{0}} \nabla^{2} \psi\left(\mathbf{r}+\mathbf{a}_{n}\right)+V_{e f f}(\mathbf{r}) \psi\left(\mathbf{r}+\mathbf{a}_{n}\right)=\varepsilon \psi\left(\mathbf{r}+\mathbf{a}_{n}\right)
$$

Considering the potential periodicity, we have

$$
\psi\left(\mathbf{r}+\mathbf{a}_{n}\right)=C_{n} \psi(\mathbf{r}),
$$

with $C_{n}$ being a constant that satisfies the normalization condition, $\left|C_{n}\right|=1$. Similarly, for another translational vector, $\mathbf{a}_{n^{\prime}} \neq \mathbf{a}_{n}$, and for the translation that results from the sum of the former two translations, $\mathbf{a}_{n^{\prime \prime}}=\mathbf{a}_{n}^{\prime}+\mathbf{a}_{n}$, we arrive at the conclusion that

$$
C_{n^{\prime}+n}=C_{n^{\prime}} \cdot C_{n}
$$

This condition is always satisfied whenever

$$
C_{n}=e^{i \mathbf{k} \cdot \mathbf{a}_{n}}
$$

where $\mathbf{k}$ is an arbitrary vector. The wavefunction of the crystal, or Bloch wave, is therefore written as:

$$
\psi(\mathbf{r})=u_{\mathbf{k}}(\mathbf{r}) e^{i \mathbf{k} \cdot \mathbf{r}}
$$


where

$$
u_{\mathbf{k}}(\mathbf{r})=u_{\mathbf{k}}\left(\mathbf{r}+\mathbf{a}_{n}\right)
$$

is a periodic function, known as the Bloch amplitude and, $e^{i \mathbf{k r}}$ is the Bloch exponential. Therefore resulting in,

$$
\psi\left(\mathbf{r}+\mathbf{a}_{n}\right)=\psi(\mathbf{r}) e^{i \mathbf{k} \cdot \mathbf{a}_{n}} .
$$

Eq. 2.11 expresses the Bloch theorem and the respective wavefunction is built by two factors: the Bloch amplitude, which is the same for any unit-cell, and the 'envelope function', which consists in an harmonic function. The parameter that distinguishes the different wavefunctions is the wavevector, k. If Eq. 2.11 is valid for one $\mathbf{k}$ wavevector, it will also be valid for a wavevector $\mathbf{k}^{\prime}$, with

$$
\begin{array}{cll}
\mathbf{k}^{\prime} & = & \mathbf{k}+\mathbf{b}_{n} \\
\left(\mathbf{b}_{n} \cdot \mathbf{a}_{n}\right) & =0,2 \pi, 4 \pi, \ldots
\end{array}
$$

The reciprocal lattice therefore can be constructed based on the vectors,

$$
\begin{aligned}
\mathbf{b}_{1} & =\frac{2 \pi}{v}\left(\mathbf{a}_{2} \times \mathbf{a}_{3}\right), \\
\mathbf{b}_{2} & =\frac{2 \pi}{v}\left(\mathbf{a}_{3} \times \mathbf{a}_{1}\right), \\
\mathbf{b}_{3} & =\frac{2 \pi}{v}\left(\mathbf{a}_{1} \times \mathbf{a}_{2}\right),
\end{aligned}
$$

where $v=\mathbf{a}_{1} \cdot\left[\mathbf{a}_{2} \times \mathbf{a}_{3}\right]$ is defined as the volume of the unit cell.

Application of Bloch's theorem will ensure that the wavefunctions will incorporate the translational periodicity of the system.

$$
\varphi_{\mathbf{k}, n}(\mathbf{r})=e^{\imath \mathbf{k} \cdot \mathbf{r}} \sum_{\mathbf{G}} c_{\mathbf{k}, n}(\mathbf{G}) e^{\imath \mathbf{G} \cdot \mathbf{r}},
$$

where $c_{\mathbf{k}, n}$ are the coefficients for the plane waves, with $\mathbf{k}$ being the wavevector and $n$ the band index; and $\mathbf{G}$ are the reciprocal lattice vectors, of the form $\mathbf{G}=m_{1} \mathbf{b}_{1}+m_{2} \mathbf{b}_{2}+m_{3} \mathbf{b}_{3}$. The electronic density is

$$
n(\mathbf{r})=\sum_{\mathbf{k}, n} \sum_{\mathbf{G}, \mathbf{G}^{\prime}} f_{\mathbf{k}, n} c_{\mathbf{k}, n}^{*}\left(\mathbf{G}^{\prime}\right) c_{\mathbf{k}, n}(\mathbf{G}) e^{\imath\left(\mathbf{G}-\mathbf{G}^{\prime}\right) \cdot \mathbf{r}}
$$


where $f_{\mathbf{k}, n}$ are the band occupation numbers. Fourier transformation held:

$$
n(\mathbf{G})=\sum_{\mathbf{k}, n} \sum_{\mathbf{G}^{\prime}} f_{\mathbf{k}, n} c_{\mathbf{k}, n}^{*}\left(\mathbf{G}^{\prime}-\mathbf{G}\right) c_{\mathbf{k}, n}\left(\mathbf{G}^{\prime}\right) .
$$

The sums over $\mathbf{k}$ must be performed over all BZ wavevectors, but this can be reduced to sums on the irreducible BZ by taking advantage of the space group symmetry of the lattice.

\section{Consequences of the Bloch Theorem}

The electronic states with $\mathbf{k}$ and $\mathbf{k}+\mathbf{b}$ (where $\mathbf{b}$ is a translational vector of the first BZ), with

$$
\varepsilon(\mathbf{k})=\varepsilon(\mathbf{k}+\mathbf{b}),
$$

defines the electron in the crystal as being a periodic function in $\mathbf{k}$. All the physically distinguishable states correspond to those $\mathbf{k}$ of the first BZ. Moreover,

$$
\varepsilon(\mathbf{k})=\varepsilon(-\mathbf{k}),
$$

which is due to the symmetry of the Schrödinger equation with respect to time-inversion symmetry

The allowed values for the $\mathbf{k}$ components are determined by the boundaryconditions in the crystal surfaces. Generally, the boundary-conditions are those proposed by Born and von Karman,

$$
\psi(\mathbf{r})=\psi(\mathbf{r}+\mathbf{L})
$$

where $\mathbf{r}$ is the position vector of a point on the crystal surface, $\mathbf{L}=\left(L_{x}, L_{y}, L_{z}\right)$, with $L_{\alpha}(\alpha=x, y, z)$ being the dimension of the crystal on the respective direction.

Applying this condition to the wavefunction of Eq. 2.11, we obtain that the quasi-momentum $(\hbar \mathbf{k})$ will vary discretely. 


\section{Quasi-free Electron Approximation}

The quasi-free electron approximation is based on the assumption that $V_{e f f}(\mathbf{r})$ is sufficiently weak to be considered as a perturbation with respect to the free electron system (non-perturbed system). This situation corresponds to admitting that electrons interact weakly with the ions of the crystal, and between themselves.

The non-perturbed wavefunction can be described by a plane-wave through

$$
\psi(\mathbf{r})=\frac{1}{\sqrt{v}} e^{i \mathbf{k r}}
$$

where $v$ is the volume of the crystal. From perturbation theory one has:

$$
\varepsilon(\mathbf{k})=\frac{\hbar^{2} k^{2}}{2 m_{0}}+\sum_{\mathbf{k}^{\prime} \neq \mathbf{k}} \frac{\left|V_{\mathbf{k k}^{\prime}}\right|^{2}}{\varepsilon_{0}(\mathbf{k})-\varepsilon_{0}\left(\mathbf{k}^{\prime}\right)},
$$

where $\varepsilon_{0}(\mathbf{k})=\hbar^{2} k^{2} / 2 m_{0}$ and $V_{\mathbf{k k}^{\prime}}$ is the matrix element of the potential $V_{\text {eff }}(\mathbf{r})$,

$$
V_{\mathbf{k} \mathbf{k}^{\prime}}=\frac{1}{v} \int_{v} V(\mathbf{r}) e^{i\left(\mathbf{k}-\mathbf{k}^{\prime}\right) \mathbf{r}} d \mathbf{r} .
$$

Eq. 2.22 determines the energy band-structure for the quasi-free electron energy spectrum.

The action of the periodic potential, with origin on the crystalline ionic lattice, is considered as a perturbation and determines the appearance of forbidden energy-bands, or energy-gap.

\subsubsection{Disordered Crystalline Systems and Surface States}

Equilibrium lattice structures are dependent on temperature and pressure. Even at temperature ranges where vibrations about a particular state are small, the entropy associated with the vibrations may be large enough to cause the ions to switch from one configuration to another. In some cases, more than one crystalline form of an element or compound may be stable at a given temperature and pressure; these are known as allotropes [27].

Systems are usually ordered at low temperatures, but upon heating, they may undergo phase transitions into less ordered states. Other forms of disorder occur when the crystalline pattern of the atoms or molecules in the solid is interrupted by crystallographic defects. 
Long range order characterizes the physical system, in which remote portions of the same sample exhibit correlated behavior. A correlation function can be expressed as $G\left(n, n^{\prime}\right)$, where $n$ displays the distance function within the system. This function decreases as the distance $\left|n-n^{\prime}\right|$ increases; when it decays exponentially to zero, the system is considered to be disordered. The system possesses long-range order [30] if the correlation function decays to a constant value at large distances.

The presence or absence of symmetry or correlation in a many-particle system is characterized through order or disorder. The degree of freedom that characterizes the order/disorder can be identified through translation, rotation, or spin states, which will consist in the characterization of a full symmetry space-group.

An ideal perfect crystal, where every atom of the same type is at the correct position, does not exist in nature. All crystals have a certain amount of impurities. Atom arrangements of a real material do not obey perfect crystalline patterns.

The atoms are relatively motionless in solids, and therefore it is difficult to eliminate any imperfections that might occur, and which may be introduced into the crystal during its growth environment, processing or application.

The fact that real materials are not perfect crystals is a fundamental advantage for material engineering. If solids were perfect crystalline structures, then its properties would be dictated solely by the composition and the crystal structure alone, restricting therefore the variety and functionality of allowed solids. The possibility of engineering crystalline solids with imperfections will allow property adaption for several combinations that modern engineering devices require. Therefore, the most important characteristics for the micro-structure of a device, are the crystalline defects, which may be manipulated to control the electronic and optical properties, according to the desired engineering application.

Moreover, considering the point defect perturbation, one may distinguish between intrinsic defects and extrinsic defects. Intrinsic defects are formed when an atom, belonging to the host crystal, is missing from a position, creating a vacancy, or when this atom occupies a void in the lattice, thus creating an interstitial site. Extrinsic defects, on the other hand, are caused by solute or foreign impurity atoms (atoms that do not belong to the host lattice).

Electrically active defects are defined as atomic configurations which give rise to electronic states in the band gap of the material. Impurities play an important 
role in physics, and their presence is inevitable in all materials, which can either take place naturally (in contact to solvents or air by oxidation or reduction processes) or can also be intentionally manipulated during the growth or the processing of the materials. Therefore considerable effort has been directed towards the study of defects in semiconductors and insulators and pragmatic strategies are engineered in order to control the defect densities, according to the potential device application of the material [31]. The level of impurities in a material is generally defined in relative terms, and the material's level of purity can only be compared as being more or less pure than some other material.

Unlike the advantages of shallow impurities to enable conducting properties, deep defects can cause unreliability, being these the main origin for electrical failure and breakdown of devices. Trapped charges in defects cause a shift in the gate threshold voltage of the transistor; it can also change with time, shifting the threshold voltage with time, thus leading to instability of operating characteristics. Also, trapped charges scatter carriers in the channel and lowers the carrier mobility [31].

The presence of impurities can also enable important effects on the mechanical, electronic and magnetic properties of materials. For example, in metal-alloys, the conduction electron spins form a magnetic bound state with the impurity atom - the Kondo effect. In superconductors, magnetic impurities yield sites for vortex defects.

The wide range of colors observed in various materials and minerals in nature, is connected to the semiconductors band-gap widths. It is accepted that the mechanism that governs color in many materials, in particular semiconductors, can be explained by band-theory. A reflective surface occurs in materials, when polished, but semiconductors are less efficient in conducting electricity than metals. In fact, semiconductors frequently act as insulators, and require special conditions in order to become conductors, with enhanced performance as temperature increases. By contrast, metals get larger resistance with increasing temperature.

A defect in a crystal structure is also said to be a color center if it induces a change in color. Color centers can be classified as electron color centers or hole color centers. The color centers cause the solid to become colored when the electronic ground state of the defect is excited to higher energy states by the absorption of visible light. Well known examples of color centers are the presence of transition metal impurities that originate the distinctive colors of gemstones in an otherwise transparent crystal lattice. Brilliant colors are com- 
monly produced, when impurities are introduced on colorless gemstones. For example, fluorite is built with alternating calcium ions $\left(\mathrm{Ca}^{2+}\right)$ and fluorine ions $\left(\mathrm{F}^{-}\right)$. Every positive calcium ion is surrounded by negative fluorine ions, and vice-versa. The electrical attraction between the positive and negative charges creates strong bonds in the crystal. In its pure form, fluorite is transparent, but the purple feature of fluorite is caused by a defect in the crystal. Fluorite occurs in a wide variety of colors, also presenting fluorescent behavior. Transparent diamonds can be colored blue or yellow when certain impurities are added. The existence of impurities in corundum can produce a deep blue sapphire. Also, the presence of chromium impurities on a colorless corundum, gives rise to a red ruby, and the colorless beryl, transforms into a green emerald. The addiction of iron impurities transforms a crystalline quartz into a violet Amethyst.

Another common and most studied impurity is interstitial hydrogen, that can be unintentionally incorporated during the growth environment [32], due to the source gases which contain hydrogen as carrier gas and in molecular beam epitaxy where hydrogen is the prevailing background impurity, exhibiting complex behaviors when introduced in materials [32].

Hydrogen can interact with other dopants, similarly to what occurs, for example in Ge, where this impurity is found to counteract electrical defects and activate neutral impurities [33]. Hydrogen can also be used to passivate the dangling bonds at the $\mathrm{SiO}_{2} / \mathrm{Si}$ interface. In many other materials, as to what happens for example in $\mathrm{GaN}$, where the neutral state, $\mathrm{H}^{0}$, is never the lowestenergy state, a negative- $U$ effect is obtained; hydrogen acts as a deep, amphoteric impurity, which always counteracts the prevailing conductivity [33]. Hydrogen can also behave as a donor dopant, for instance in $\mathrm{ZnO}$, enabling a shallow defect level close to the conduction band. These findings motivate the research of the hydrogen behavior in many semiconductors and oxides, where it can influence their electrical properties [33].

The presence of impurity atoms in semiconductors, perturbs the crystalline potential, which may produce localized energy levels located in the band gap. More relevant impurities are those that create localized levels close to the ends of the conduction band (CB) and/or the valence band (VB); these are known as shallow levels. Impurities that create localized levels near the $\mathrm{CB}$ are called donors, and those which give rise to levels near the VB are acceptor levels.

One way to control the conductivity of semiconductors is to add impurities and/or imperfections to the system (doping). This can be done either by replacing some of the semiconducting atoms by atoms of other elements of differ- 
ent potential (substitutional impurities), or by adding impurities on interstitial sites in an energetically favorable configuration of the lattice (interstitial impurities). The resulting conductivity is referred to as extrinsic conductivity. The energy levels related to impurities, are not extended states, but are localized, and can give rise to an impurity discrete level. The reason is that impurity atoms are distant from each other (when doping concentration is low), of the order of several lattice parameters, thus being very weakly interacting with each other.

As already mentioned above, the presence of impurities leads to the emergence of localized states. The number of states, per unit volume, is equal to the concentration of impurity atoms. Considering the example of donor states, we have,

$$
g(E)=N_{i} \delta\left(E_{c}-E_{i}\right)
$$

where $N_{i}$ is the impurity concentration and $E_{i}$ is the level in the gap created by the impurity (in this case from the donor type) and $E_{c}$ the energy of the conduction-band minimum [29]. The excited states are already close to the CB, where the density of states is higher, so these do not exhibit fundamental importance to the electronic statistics.

Expression 2.24 is only valid if the impurity concentration is not too high. Under these conditions, the average distance between the impurity sites is $\approx N_{i}^{-1 / D}$, (where $D$ is the dimension of the material), which is higher than the effective Bohr radius, $a=\varepsilon_{0} \hbar^{2} m^{-1} e^{-2}$, where $\varepsilon_{0}$ is the dielectric constant of the material and $m$ is the effective mass of the charge carriers (this parameter indicates the number/distance of unit cells that can be 'reached' by the charge carriers, around the impurity atom).

The most simplistic model to predict localized energy levels is by employing the analog of the hydrogen model, the hydrogenoic model. When the above mentioned distance, $D$, becomes comparable to $a$, then each atom of the impurity will 'feel' the presence of other impurity sites, thus arising an interaction between the electrons located in different sites of the impurity atoms. Another effect contributing to the energy dispersion of the localized levels is the Coulomb interaction, originated from an ionized impurity. Owing that the distance fluctuates between neighboring donors, the energy of this interaction is random. The outcome is the formation of an impurity-band, and the formation of this bandwidth can be rather complex and therefore difficult to calculate the respective density of states (DOS) [34].

In order to study the doping effects on a semiconductor material, a good control of the doping concentration must be quantitatively and qualitatively ensured. 
In a highly-doped semiconductor, the impurity-band, whilst being a set of states, each of which is created by a single donor/acceptor atom, can disappear due to the shielding effect produced by free charge carriers. In this high-limit doping, the DOS becomes continuous and forms a tail that extends into the gap.

\section{Surface States}

The presence of surface and interface states give rise to electrical potential profiles at the surface, which are located within the gap region, between the $\mathrm{CB}$ and VB. The surface states may arise due to either surface contamination, or imperfections at the surface, or due to dangling bonds associated with incomplete covalent bonding $[35,36,37]$. The surface breaks the translational symmetry of the perfect crystal, thus leading to the appearance of the known Tamm levels [38, 39]. Furthermore, due to favorable adsorption observed on the surfaces, and also due to the appearance of dangling bonds, unexpected energy levels emerge in the electronic band structure, when compared to the bulk crystal. On the surface of any sample of a semiconducting material, many of these well known localized states exist and are called surface states.

The profile of the surface electric potential is associated with the presence of surface states, which give rise to a surface electric field. Indeed, Tamm (1932) [38] and Shockley (1939) [40] demonstrated, that this electrical potential profiles are in fact related to the presence of surface states [38, 40, 41, 42, 43]. The characterization of the electric potential is very important to understand the physical phenomena which occurs within these semiconducting structures. Interesting applications may originate from this phenomena, for example, in the construction of more rapid and sensitive electronic devices and to the catalysis phenomenon.

Some of these localized surface states may appear within the theoretically predicted gap of the perfect crystal; if the states overlap with the permitted bands they are called resonant states. Surface states express an almost-continuous distribution within the gap. These states may attract electrons of the bulk ('semiconductor volume') if its occupation is energetically more favorable. Due to this effect, the surface Fermi level can take a different position with respect to the corresponding bulk Fermi level - pinning of Fermi level. Also, the bottom of the $\mathrm{CB}$ and the top of the $\mathrm{VB}$ are also shifted relative to their original positions of the bulk semiconductor. 
The electric potential profile can be calculated based on the charge distributions within the sample. Experimental techniques, such as modulation spectroscopy techniques $[44,45,46]$, aid in the determination of the electrical potential surface. One example is the photo-reflectance technique used to characterize and measure the electronic band structure and optical response of several semiconducting materials [44, 45, 46].

\section{Concentration of Charge Carriers as a Function of the Concentration of Impurities and Temperature}

The control of the charge carriers concentration (electron or holes) of a semiconductor is an important requirement to obtain the desirable electrical properties for a given application. The physical effects of the impurity density/concentration are temperature dependent and system dependent (semiconductor parameters), determined by the DOS and the probability occupation.

Some impurities can create simultaneously donor levels, $E_{d}$, close to the CB edge and acceptors, $E_{a}$, close to the top of the VB. Such materials are called compensated semiconductors which contain both donor and acceptor impurities in comparable concentrations. It should however be noteworthy, that the compensation significantly alters the position of the Fermi level for low temperatures.

At temperatures close to absolute zero, the Fermi level splits the empty states from the occupied states. For the impurity band, wherein there is a level distribution, the Fermi level will lie at the center of this band. This situation is similar to that found on metals that have partially filled bands. In fact, under certain conditions, the conductivity on the impurity band may have a metallic character, depending on the position where the Fermi level lies [34]. This phenomenon is known as the Mott-Anderson transition [47, 48].

This effect not only takes place on heavily doped-semiconductors but it has also been observed on amorphous materials. From a theoretical point of view, this is a fundamental problem of localization/delocalization of electronic states in disordered systems. Currently, it is widely accepted, the existence of a characteristic energy in the electronic spectrum that borders between localized and delocalized states. This energy depends on the degree of disorder and is called mobility threshold [47]. The Mott-Anderson transition occurs when the Fermi level crosses the threshold mobility. One may consider that a material in which the Fermi level $(T \rightarrow 0)$ is located either within the gap or between localized states, has a dielectric character. By contrast, a material is metallic 
if the Fermi level (for $T \rightarrow 0$ ) is located between the allowed electronic states and the delocalized states. Thus, from this point of view, a heavily doped and compensated semiconductor can be regarded as a metal. However, typically for temperatures higher than $10 \mathrm{~K}$, the effect of the impurity bands on the electrical conductivity, is negligible.

The degree of disorder can be defined by a disorder parameter, $\Delta_{d}$, which measures the width of probability distribution relative to the average distance between the impurities for disorder sites and relative to the disorder amplitude band-width. The mean free path, $l$, which at $T=0$ is only the scattering characterization, is a function of $\Delta_{d}$ and the dimension of the system. For weak disorder, $l$ will be large compared to the wavelength $\lambda=2 \pi / k_{F}$ of the electrons, $l\left(\Delta_{d}\right) \gg \lambda$. In this regime of weak localization, the electrons follow classical trajectories between collisions, so disorder only gives rise to quantum corrections to the conductivity, but is not sufficient to trap the electrons $[49,50,51]$. The latter occurs when $\Delta_{d}$ is large such that $l\left(\Delta_{d}\right) \approx \lambda$, to which the notion of wavefunction becomes meaningless. In this regime of strong localization, Anderson localization [48, 49, 50], the wavefunction is exponentially localized [52, 53], $|\phi(\mathbf{r})|^{2} \approx \exp (-|\mathbf{r}| / \xi)$, where $\xi=\xi\left(\Delta_{d}\right)$ is the localization length [54]. Since the notion of wave disappears in this regime, the condition $\lambda>l$ has no meaning; this is known has the Ioff-Regel-Mott criterion [51, 52].

The transition from weak to strong localization is a metal-insulator transition called Anderson transition [52, 53, 47], and which is characterized by a critical value of disorder, $\Delta_{c}$. For weak localization we have $\Delta<\Delta_{c}$ and $\xi \rightarrow \infty$. As already mentioned, for disordered sites, which physically are more important, $\Delta$ is measured in units of the average distance $c^{-1 / D}$ between the impurities, $c$ being the impurity concentration. Therefore, in this case, the metal-insulator transition is also characterized by a critical concentration $c_{c r}$. Moreover, there exists a critical dimension $D_{c r}$ such that $\xi<\infty$ for $D<D_{c r}$.

According to Mott [52, 53], localized and extended electron/donor states are energetically separated by the mobility edge, $\varepsilon_{c}[47]$. There is conduction only if the Fermi energy, $\varepsilon_{F}$, lies at the extended part of the spectrum above $\varepsilon_{c}$. The conductivity will depend on the degree of disorder $\Delta$ as well as on the impurity concentration $c[52,53,47,55]$.

Another major topic relevant for the study of impurities, is related to the physics of phase transitions. In the study of phase transition theory, it is well known that the stable new phase requires a finite-size domain for the creation of a new phase; this is the establishment of a critical size achievement of a nucleus formation, such that the energetic cost will be lower at the point defect. This 
threshold size is often lower at an impurity site, which is the major cause for inducing phase transitions.

\subsection{Tight-Binding Approximation}

The state of the electron in a crystal is considered to be very similar to its atomic state.

Such an approach is acceptable for the core electrons, for which the wavefunction is localized in the vicinity of the atomic nucleus. Within this condition, the interaction with the other atoms is quite weak. The wavefunction may be obtained through the linear combination of atomic orbitals in the form:

$$
\psi(\mathbf{r})=\sum_{j} a_{j} \phi\left(\mathbf{r}-\mathbf{R}_{j}\right)
$$

where $a_{j}$ is the coefficient which corresponds to $\phi_{j}=\phi\left(\mathbf{r}-\mathbf{R}_{j}\right)$, and represents the atomic orbital of the atom positioned at point $\mathbf{R}_{j}$. Each $\phi_{j}$ obeys the Schrödinger equation such that:

$$
-\frac{\hbar^{2}}{2 m_{0}} \nabla^{2} \phi_{j}+U_{j} \phi_{j}=\epsilon_{j} \phi_{j}
$$

with $U_{j}$ being the atomic potential and $\epsilon_{j}$ an atomic energy level. By replacing Eq. 2.25 with Eq. 2.5, one obtains

$$
\sum_{j}-a_{j}\left(\frac{\hbar^{2}}{2 m_{0}} \nabla^{2}+a_{j}\left(V-U_{j}\right)+a_{j} U_{j}\right) \phi_{j}=\varepsilon \sum_{j} a_{j} \phi_{j} .
$$

According to Eq. 2.26, and taking into account that all atoms are the same with energy level $\epsilon$, one obtains

$$
\sum_{j}\left(V-U_{j}\right) a_{j}-(\varepsilon-\epsilon) a_{j} \phi_{j}=0 .
$$

By multiplying Eq. 2.28 by the function $\phi_{j^{\prime}}^{*}$ and integrating, we have:

$$
\sum_{j} a_{j}\left(\int\left(V-U_{j}\right) \phi_{j} \phi_{j^{\prime}}^{*} d \mathbf{r}-(\varepsilon-\epsilon) \int \phi_{j} \phi_{j^{\prime}}^{*} d \mathbf{r}\right)=0 .
$$

The integrals $A\left(R_{j j^{\prime}}\right)=\int\left(V-U_{j}\right) \phi_{j} \phi_{j^{\prime}}^{*} d \mathbf{r}$ e $S\left(R_{j j^{\prime}}\right)=\int \phi_{j} \phi_{j^{\prime}}^{*} d \mathbf{r}$, only depend on the atom distances, $j$ and $j^{\prime}$. For the wavefunction of Eq. 2.25, obeying 
the Bloch theorem, the coefficients $a_{j}$ are written the form:

$$
a_{j}=e^{i \mathbf{k R}_{j}} .
$$

Hence,

$$
\phi(\mathbf{r})=\sum_{j} e^{i \mathbf{k} \mathbf{R}_{j}} \phi\left(\mathbf{r}-\mathbf{R}_{j}\right)=\sum_{j} e^{i \mathbf{k}\left(\mathbf{R}_{j}-\mathbf{r}\right)} \phi\left(\mathbf{r}-\mathbf{R}_{j}\right) e^{i \mathbf{k r}},
$$

where $\sum_{j} e^{i \mathbf{k}\left(\mathbf{R}_{j}-\mathbf{r}\right)} \phi\left(\mathbf{r}-\mathbf{R}_{j}\right)=u_{\mathbf{k}}(\mathbf{r})$. The wavefunction will therefore have the compatible form to the Bloch theorem of Eq. 2.5. By replacing Eqs. 2.30 and 2.28 and by multiplying with $e^{-i \mathbf{k R}_{j^{\prime}}}$, the following result is obtained:

$$
\varepsilon=\epsilon+\frac{\sum_{j} e^{i \mathbf{k}\left(\mathbf{R}_{j}-\mathbf{R}_{j^{\prime}}\right)} A\left(R_{j j^{\prime}}\right)}{\sum_{j} e^{i \mathbf{k}\left(\mathbf{R}_{j}-\mathbf{R}_{j^{\prime}}\right)} S\left(R_{j j^{\prime}}\right)},
$$

or, by modifying the sum indexes by $m=j-j^{\prime}$,

$$
\varepsilon=\epsilon+\frac{\sum_{m} e^{i \mathbf{k}\left(\mathbf{R}_{m}\right)} A\left(R_{m}\right)}{\sum_{m} e^{i \mathbf{k}\left(\mathbf{R}_{m}\right)} S\left(R_{m}\right)} .
$$

For a perfect crystal one considers that $\epsilon_{j}=\epsilon$ and $\mathbf{R}_{m}=\mathbf{R}_{j}-\mathbf{R}_{j^{\prime}}$, which is also a translational vector of the lattice.

By admitting that the overlap of the wavefunctions is small, it is admissible to employ the following approximation:

$$
\begin{aligned}
& S\left(R_{m}\right)= \begin{cases}1 & \text { for } \quad \mathbf{R}_{m}=0 \\
0 & \text { for other conditions }\end{cases} \\
& A\left(R_{m}\right)=\left\{\begin{array}{l}
A_{0} \quad \text { for } \quad \mathbf{R}_{m}=0 \\
A_{1} \quad \text { for } \quad \mathbf{R}_{m}=\mathbf{a} \quad\left(1^{\text {st }} \text { neighbors }\right) \\
0 \quad \text { for other conditions }
\end{array}\right.
\end{aligned}
$$

In the calculation of $A\left(\mathbf{R}_{j j^{\prime}}\right)$, the term $\phi_{j} \phi_{j^{\prime}}^{*}$, of reduced value, is compensated by the large difference found between the crystalline and atomic potentials $V-U_{j}$, which are distanced from atom $j$.

Therefore,

$$
\varepsilon(\mathbf{k})=\epsilon+A_{0}+A_{1} \sum_{N} e^{i \mathbf{k} \mathbf{R}_{m}}
$$


where $N$ refers to the sum of the next-neighbors.

An example can be given for a square lattice in the form,

$$
\varepsilon(\mathbf{k})=\epsilon+A_{0}+A_{1}\left(\cos k_{x} a+\cos k_{y} a\right)
$$

As one may confirm from Eq. 2.36, the width of the band will depend of $A_{1}$, and this parameter is dependent upon the degree of overlap of the atomic orbitals between the first next-neighbors.

\section{Tight-Binding Model in Second Quantization Formulation}

The tight-binding formulation of band electrons can also be easily implemented in second quantization language that provides a intuitive interpretation. For simplicity we restrict here to the single-orbital case and define the following Fermionic operators,

$$
\begin{gathered}
\hat{c}_{j, s}^{\dagger} \quad \begin{array}{c}
\text { creates an electron of spin } s \text { on lattice site } \mathbf{R}_{j}, \\
\hat{c}_{j, s}
\end{array} \text { annihilates an electron of spin } s \text { on lattice site } \mathbf{R}_{j},
\end{gathered}
$$

We introduce the following Hamiltonian,

$$
H=\sum_{j, s} \varepsilon_{0} \hat{c}_{j, s}^{\dagger} \hat{c}_{j, s}+\sum_{i, j} t_{i j} \hat{c}_{i, s}^{\dagger} \hat{c}_{j, s}
$$

with $t_{i j}=t_{j i}$ real. These coefficients $t_{i j}$ are called "hopping matrix elements", since $\hat{c}_{i, s}^{\dagger} \hat{c}_{j, s}$ annihilates an electron on site $\mathbf{R}_{j}$ and creates one on site $\mathbf{R}_{i}$. In this way, an electron moves (hops) from $\mathbf{R}_{j}$ to $\mathbf{R}_{i}$. Thus, this Hamiltonian represents the "kinetic energy" of the electron. Let us now diagonalize this Hamiltonian by following Fourier transformation, equivalent to the transformation between Bloch and Wannier functions,

$$
\hat{c}_{j, s}^{\dagger}=\frac{1}{\sqrt{N}} \sum_{\mathbf{k}} \hat{c}_{\mathbf{k}, s}^{\dagger} e^{-i \mathbf{k} \cdot \mathbf{R}_{j}} \quad \text { and } \quad \hat{c}_{j, s}=\frac{1}{\sqrt{N}} \sum_{\mathbf{k}} \hat{c}_{\mathbf{k}, s} e^{i \mathbf{k} \cdot \mathbf{R}_{j}}
$$

where $\hat{c}_{\mathbf{k}, s}^{\dagger}\left(\hat{c}_{\mathbf{k}, s}\right)$ creates (annihilates) an electron in the Bloch state with pseudo-momentum $\mathbf{k}$ and spin $s$. Inserting this into the Hamiltonian 2.38 leads to

$$
H=\frac{1}{N} \sum_{\mathbf{k}, \mathbf{k}^{\prime}, s}\left[\sum_{i} \varepsilon_{0} e^{i\left(\mathbf{k}-\mathbf{k}^{\prime}\right) \cdot \mathbf{R}_{i}}+\sum_{i, j} t_{i j} e^{i \mathbf{k} \cdot \mathbf{R}_{j}-\mathbf{k}^{\prime} \cdot \mathbf{R}_{i}}\right] \hat{c}_{\mathbf{k}^{\prime}, s}^{\dagger} \hat{c}_{\mathbf{k}, s}=\sum_{\mathbf{k}, s} \varepsilon_{\mathbf{k}} \hat{c}_{\mathbf{k}, s}^{\dagger} \hat{c}_{\mathbf{k}, s}
$$


where $\hat{c}_{\mathbf{k}^{\prime}, s}^{\dagger} \hat{c}_{\mathbf{k}, s}=\hat{n}_{\mathbf{k}^{\prime}, s}^{\dagger}$ constitutes the number operator for electrons. The band energy is the same as obtained above from the tight-binding approach. The Hamiltonian 2.38 is used for the Hubbard model where a real-space formulation is helpful. The real-space formulation of the kinetic energy allows also for the introduction of disorder, i.e. non-periodicity which can be most straightforwardly implemented by site dependent potentials $\varepsilon_{0} \rightarrow \varepsilon_{0 i}$ and by spatially (bond) dependent hopping matrix elements $t_{i, j}=t\left(\mathbf{R}_{i}, \mathbf{R}_{j}\right) \neq t\left(\mathbf{R}_{i}-\mathbf{R}_{j}\right)$.

The Hubbard model (describes electronic states) is based on the tight-binding approximation, and takes into account if a site is doubly occupied. The Hubbard model, has the form

$$
H=-t \sum_{\langle i, j\rangle, s}\left(\hat{c}_{i, s}^{\dagger} \hat{c}_{j, s}+h . c\right)+U \sum_{i} \hat{n}_{i, \uparrow} \hat{n}_{i, \downarrow}
$$

where we consider hopping between nearest neighbors only, via the matrix element $-t$. The $\hat{c}_{i, s}^{\dagger}$ are real-space field operators on the lattice (site index $i$ ) and $\hat{n}_{i, s}=\hat{c}_{i, s}^{\dagger} \hat{c}_{i, s}$ is the density operator. In the case of half filling, it results in one electron per site on average. There are two evident limiting cases:

- Insulating atomic limit: $t=0$. The ground state has exactly one electron on each lattice site.

- Metallic band limit: We set $U=0$. The electrons are independent and move freely via hopping processes. The band energy is found through a Fourier transform of the Hamiltonian. With 2.39 we can rewrite

$$
-t \sum_{\langle i, j\rangle, s}\left(\hat{c}_{i, s}^{\dagger} \hat{c}_{j, s}+h . c\right)=\sum_{\mathbf{k}, s} \varepsilon_{\mathbf{k}} \hat{c}_{\mathbf{k}, s}^{\dagger} \hat{c}_{\mathbf{k}, s}
$$

where $\varepsilon_{\mathbf{k}}=-t \sum_{\mathbf{a}} e^{i \mathbf{k} \cdot \mathbf{a}}$ and the sum runs over all vectors a connecting nearest neighbors.

\subsection{Green Function's Methods for Description of Disorder Materials}

The method of the Green's functions (GF) is one of the most powerful and universal in theoretical physics. The concept of GF was developed at the XIX century, in the context of classical physics associated with linear differential 
problems in partial derivatives and theory of propagation of heat, electrostatics, magnetostatics, wave propagation, etc. In the basic formulation is considered the uniform system:

$$
\widehat{L} u(\mathbf{r}, t)=0,
$$

were $\widehat{L}$ is a linear differential operator, usually with 1 st and 2 nd order derivatives, and $u(\mathbf{r}, t)$ is the requested function. For this problem, the GF $g(\mathbf{r}, t)$ is defined as the solution of the associated equation:

$$
\widehat{L} g(\mathbf{r}, t)=\delta(\mathbf{r}) \delta(t)
$$

Also these functions are known as fundamental solutions to the problem, Eq. 2.43. The importance of the GF lies in the possibility of obtaining solutions to a non-uniform problem related to the equation 2.43:

$$
\widehat{L} u(\mathbf{r}, t)=f(\mathbf{r}, t)
$$

where $f(\mathbf{r}, t)$ is an arbitrary function. Then the solution for Eq. 2.44 has the form:

$$
u(\mathbf{r}, t)=u_{0}(\mathbf{r}, t)+\int g\left(\mathbf{r}-\mathbf{r}^{\prime}, t-t^{\prime}\right) f\left(\mathbf{r}^{\prime}, t^{\prime}\right) d \mathbf{r}^{\prime} d t^{\prime},
$$

where $u_{0}(\mathbf{r}, t)$ is a solution of Eq. 2.43. This construction also indicates the route for the treatment of the disorder corresponding problem of Eq. 2.43:

$$
(\widehat{L}+\widehat{V}) u(\mathbf{r}, t)=0
$$

leading it to the form similar to Eq. 2.44: $\widehat{L} u=-\widehat{V} u$, and writing the solution as an integral equation for $u(\mathbf{r}, t)$ :

$$
u(\mathbf{r}, t)=u_{0}(\mathbf{r}, t)-\int g\left(\mathbf{r}-\mathbf{r}^{\prime}, t-t^{\prime}\right) V u\left(\mathbf{r}^{\prime}, t^{\prime}\right) d \mathbf{r}^{\prime} d t^{\prime} .
$$




\section{Perturbation Theory}

The one-particle Hamiltonian, $\mathcal{H}$, can be separated into an unperturbed part, $\mathcal{H}_{0}$, and a perturbation, $\mathcal{H}_{1}$, where $\mathcal{H}=\mathcal{H}_{0}+\mathcal{H}_{1}$. Assuming we can solve for the GF $\mathcal{G}_{0}$ of $\mathcal{H}_{0}$, we find $\mathcal{G}$ of $\mathcal{H}$, noting that $\mathcal{G}_{0}(\varepsilon)=\left(\varepsilon-\mathcal{H}_{0}\right)^{-1}$ and $\mathcal{G}(\varepsilon)=(\varepsilon-\mathcal{H})^{-1}$, we obtain the called Dyson 's equation

$$
\mathcal{G}=\mathcal{G}_{0}+\mathcal{G}_{0} \mathcal{H}_{1} \mathcal{G}
$$

Often $H_{1}\left(\mathbf{r}_{1}, \mathbf{r}_{2}\right)$ is a local potential which can be written $\delta\left(\mathbf{r}_{1}-\mathbf{r}_{2}\right) V\left(\mathbf{r}_{1}\right)$; then Eq. 2.45 written in terms of the coordinates, $\mathbf{r}$-representation, becomes

$$
G\left(\mathbf{r}, \mathbf{r}^{\prime} ; \varepsilon\right)=G_{0}\left(\mathbf{r}, \mathbf{r}^{\prime} ; \varepsilon\right)+\int d \mathbf{r}_{1} G_{0}\left(\mathbf{r}, \mathbf{r}_{1} ; \varepsilon\right) V\left(\mathbf{r}_{1}\right) G\left(\mathbf{r}_{1}, \mathbf{r}^{\prime} ; \varepsilon\right)
$$

which is a linear inhomogeneous integral equation with a kernel of the form $G_{0}\left(\mathbf{r}, \mathbf{r}^{\prime} ; \varepsilon\right) V\left(\mathbf{r}_{1}\right)$, the Eq. 2.45, in the momentum, k-representation is

$$
G\left(\mathbf{k}, \mathbf{k}^{\prime} ; \varepsilon\right)=G_{0}\left(\mathbf{k}, \mathbf{k}^{\prime} ; \varepsilon\right)+\sum_{\mathbf{k}_{1}, \mathbf{k}_{2}} G_{0}\left(\mathbf{k}, \mathbf{k}_{1} ; \varepsilon\right) H_{1}\left(\mathbf{k}_{1}, \mathbf{k}_{2}\right) G\left(\mathbf{k}_{2}, \mathbf{k}^{\prime} ; \varepsilon\right),
$$

\section{Methods of GFs in Many Body Physics}

There are several types of GF, used in many body physics: temporal, thermal (Matsubara), functions out of equilibrium (Keldysh), etc. For our purposes, we will use the time functions. This GF is defined as:

$$
\left\langle\left\langle\hat{A}(t) \mid \hat{B}\left(t^{\prime}\right)\right\rangle\right\rangle=-i \theta\left(t-t^{\prime}\right)\left\langle\left[\hat{A}(t), \hat{B}\left(t^{\prime}\right)\right]_{ \pm}\right\rangle,
$$

where $\hat{A}(t)$ and $\hat{B}(t)$ are operators in the Heisenberg representation, $\langle\cdots\rangle$ is the quantum-statistical average with the corresponding Hamiltonian, and \pm are the commutation relations, for the operators A, B of Bose or Fermi type, respectively. These relationships corresponds to the kinds of statistics that the particles obey. Considering a general problem, the operator $\hat{\mathcal{H}}$ is the Hamiltonian of a considered system; and the large canonical ensemble, $\hat{\mathcal{H}}=\hat{H}-\mu \hat{N}$, where, $\hat{N}$ is the operator for the number of particles and $\mu$ is the chemical potential. The function $\theta\left(t-t^{\prime}\right)$ is defined as follows: $\theta(t)=1$ for $t>0, \theta(t)=0$ for $t<0$. Using the Boltzmann constant, $\beta=1 / K_{B} T$ (referred 
to the absolute temperature), we can calculate any thermodynamic value, for a quantity $Q$,

$$
\langle Q\rangle=\frac{\operatorname{Tr} \hat{Q} \exp (-\beta \hat{\mathcal{H}})}{\operatorname{Tr} \exp (-\beta \hat{\mathcal{H}})}
$$

The operators $\hat{A}(t)$ and $\hat{B}(t)$ in Eq. 2.47 are Heisenberg operators satisfying the equations of motion $i \dot{\hat{A}}=[\hat{A}, \hat{\mathcal{H}}]$. In this way Eq. 2.47 results in the motion equation corresponding to $\mathrm{GF}\left\langle\left\langle\hat{A}(t) \mid \hat{B}\left(t^{\prime}\right)\right\rangle\right\rangle$.

$$
i \frac{d}{d t}\left\langle\left\langle\hat{A}(t) \mid \hat{B}\left(t^{\prime}\right)\right\rangle\right\rangle=\delta\left(t-t^{\prime}\right)\left\langle\left[\hat{A}(t), \hat{B}\left(t^{\prime}\right)\right]_{ \pm}\right\rangle+\left\langle\left\langle[\hat{A}(t), \hat{\mathcal{H}}] \mid \hat{B}\left(t^{\prime}\right)\right\rangle\right\rangle .
$$

Considering Fourier transform

$$
\langle\langle\hat{A} \mid \hat{B}\rangle\rangle_{\varepsilon}=\int_{-\infty}^{\infty}\left\langle\left\langle\hat{A}(t) \mid \hat{B}\left(t^{\prime}\right)\right\rangle\right\rangle e^{-i \varepsilon t} d t
$$

given that $\left\langle\left\langle\hat{A}(t) \mid \hat{B}\left(t^{\prime}\right)\right\rangle\right\rangle$ is just a function of the difference $t-t^{\prime}$ and its inversion

$$
\left\langle\left\langle\hat{A}(t) \mid \hat{B}\left(t^{\prime}\right)\right\rangle\right\rangle=\int_{-\infty}^{\infty}\langle\langle\hat{A} \mid \hat{B}\rangle\rangle_{\varepsilon} e^{-i \varepsilon\left(t-t^{\prime}\right)} d \varepsilon
$$

whose energy argument $\varepsilon$ is understood as $\varepsilon-i 0$ and $\langle\{A(t), B(0)\}\rangle$ is the quantum statistical average with Hamiltonian $H$ of the anticommutator of Heisenberg operators, we obtain the equation of motion corresponding to Eq.2.49:

$$
\varepsilon\left\langle\left\langle\hat{A}(t) \mid \hat{B}\left(t^{\prime}\right)\right\rangle\right\rangle_{\varepsilon}=\frac{i}{2 \pi}\left\langle[\hat{A}, \hat{B}]_{ \pm}\right\rangle+\left\langle\left\langle[\hat{A}, \hat{\mathcal{H}}] \mid \hat{B}\left(t^{\prime}\right)\right\rangle\right\rangle_{\varepsilon} .
$$

The usefulness of GFs consists in that these are related to observable quantities, expressed by so-called correlation functions (or correlators): $\left\langle\hat{A}(t) \hat{B}\left(t^{\prime}\right)\right\rangle$. Therefore correlators account for the mean values of operators (generally different) for different times, and any observable value may be displayed in this way. 
To describe the dynamics of the many particle system, we distinguish two types of time GFs and their corresponding Fourier transforms,

$$
\begin{aligned}
\langle\langle\hat{A} \mid \hat{B}\rangle\rangle_{\varepsilon+i \delta}^{( \pm)} & =-i \int_{0}^{\infty} e^{i(\varepsilon+i \delta) t}\left\langle[\hat{A}(t), \hat{B}(0)]_{ \pm}\right\rangle d t \\
\langle\langle\hat{A} \mid \hat{B}\rangle\rangle_{\varepsilon-i \delta}^{( \pm)} & =i \int_{-\infty}^{0} e^{i(\varepsilon-i \delta) t}\left\langle[\hat{A}(t), \hat{B}(0)]_{ \pm}\right\rangle d t
\end{aligned}
$$

known as retarded and advanced GFs, respectively. The average product of operators can be expressed through the corresponding GF [56], by using the spectral theorem,

$$
\langle\hat{A}, \hat{B}\rangle=\frac{1}{2 \pi} \int_{-\infty}^{\infty} \frac{d \varepsilon}{e^{\beta(\varepsilon-\mu)} \pm 1} \lim _{\delta \rightarrow 0} i\left[\langle\langle\hat{B} \mid \hat{A}\rangle\rangle_{\varepsilon+i \delta}^{( \pm)}-\langle\langle\hat{B} \mid \hat{A}\rangle\rangle_{\varepsilon-i \delta}^{( \pm)}\right]
$$

Note that the equation of motion is the same for the retarded and advanced GFs; These two functions reflect the analytical properties of the observable averages, being the retarded GF $\langle\langle\hat{A} \mid \hat{B}\rangle\rangle_{\varepsilon}$ defined in the upper half-plane $\varepsilon$ (complex energy plane) and advanced GF defined in the lower half-plane. For the system in equilibrium, these functions are related to the correlations between quantum states different times (past and future), respectively.

\subsection{Density Functional Theory}

Theoretical chemists and physicists, following the path of the Schrödinger equation, have become accustomed to think in a truncated Hilbert space of single particle orbitals. DFT provides a complementary perspective. It focuses on the quantities in the real 3-dimensional coordinate space, principally on the electron density $n(r)$ of the ground state. These quantities are physical, independent of representation, and easily visualizable even for very large systems."

Walter Kohn's, Nobel Lecture

Density Function Theory (DFT) is an electronic structure method based on the fundamental laws of quantum mechanics and was initially developed by Hohenberg, Kohn and Sham in 1965 [57, 58]. DFT is currently one of the 
most successful approaches to compute the ground-state properties of bulk and finite-sized systems.

Some electronic structure methods, based on the expansion of the wave-function in Slater determinants, attempt to arrive at approximate solutions to the Schrödinger equation of $3 N$ interacting electrons moving in an external potential. The problem with these methods is the huge computational effort, making these methods virtually impossible to efficiently apply to larger and more complex systems. On the other hand, DFT uses a one-body density as fundamental variable. The density, $n(\mathbf{r})$, is a function of only three spatial coordinates thus making DFT computationally more feasible even for large systems.

Within this approach, the electronic orbitals are obtained as solutions of a set of Schrödinger-like equations, referred to as the Kohn-Sham equations, for which the potential terms depend solely on the electron density, $n(\mathbf{r})$. The KohnSham method assumes that for each real and interacting ground-state density, an auxiliary and non-interacting electron system should exist and possessing the same ground-state density.

The Kohn-Sham equations can be written as:

$$
\left[-\frac{\nabla^{2}}{2}+v_{\mathrm{KS}}[n(\mathbf{r})]\right] \varphi_{i}(\mathbf{r})=\epsilon_{i} \varphi_{i}(\mathbf{r}),
$$

where $v_{\mathrm{KS}}$ is the Kohn-Sham potential and $n$ is defined in terms of the KohnSham wave-functions, and constructed by summing over all the occupied orbitals:

$$
n(\mathbf{r})=\sum_{i}^{o c c}\left|\varphi_{i}(\mathbf{r})\right|^{2}
$$

The Kohn-Sham potential can be defined as the sum of three terms [59]:

- The external potential, $v_{\text {ext }}$, representing the Coulomb attraction between the bare nucleus and the electrons;

- the Hartree term, $v_{\text {Hartree }}$, which is the electrostatic energy of the electron in the field generated by the total density;

- and the exchange-correlation (xc) potential, $v_{\mathrm{xc}}$ 
following the form

$$
v_{\mathrm{KS}}[n(\mathbf{r})]=v_{\mathrm{ext}}(\mathbf{r})+v_{\text {Hartree }}[n(\mathbf{r})]+v_{\mathrm{xc}}[n(\mathbf{r})] .
$$

The xc potential (last term of Eq. 2.56), takes the form of a functional derivative of the non-interacting electronic density, such that:

$$
v_{\mathrm{xc}}[n(\mathbf{r})]=\frac{\delta E_{\mathrm{xc}}[n(\mathbf{r})]}{\delta n(\mathbf{r})} .
$$

The electron exchange term describes the wave-function of indistinguishable particles that is subject to exchange symmetry when two particles are exchanged. For any particle characterized by Fermi-Dirac statistics with halfinteger spin (fermions), i.e. electrons, this effect undergoes the Pauli exclusion principle, preventing two parallel-spin particles from being found at the same point in space (i.e. orbital). The exchange term lowers the energy by keeping the electrons of the same spin away from each other, thus reducing the Coulomb repulsion [60]. Correlation energy, on the other hand, results from the collective behaviour of electrons to screen and decrease the Coulombic interaction; correlations become more pronounced for opposite spins since they are most likely to occupy nearby locations [60,61].

DFT is an exact method, being the only approximation the treatment of the non-trivial many-body effects, which are grouped into the xc functional. This functional is obtained through approximations, and currently there are many different forms which are grouped into different ladder rungs as a function of chemical accuracy.

\section{Expansion of the Kohn-Sham Wave-functions in a Plane-Wave Basis}

The Kohn-Sham wave-functions can be expanded using different numerical basis sets, being the most natural and simple method to treat periodic systems the plane-wave expansion. With plane-waves the Kohn-Sham equations can then be solved in momentum space, and by employing Bloch's theorem the wave-functions will satisfy the translational periodicity of the system.

Two technical convergence parameters need to be adjusted for periodic calculations. One of these conditions is the Brillouin-zone (BZ) sampling which replaces the integration of the periodic functions over reciprocal space. To numerically evaluate the integrals, a weighted sum over a subset of $\mathbf{k}$-points is 
performed. By taking advantage of the space-group symmetry of the lattice, it is possible to reduce these sums to the irreducible BZ [59], thus generating a finite set of representative reducible $\mathbf{k}$-vectors.

The second convergence parameter that should be considered is the cut-off radius, which is set to truncate the sums over the reciprocal lattice vectors. The cut-off energy is given by $E_{\text {cutoff }}=G_{\max }^{2} / 2$, where $G_{\max }$ is defined as the radius of the sphere that contains all plane-waves up to the cut-off within a volume, $V_{\text {sphere }}=(4 \pi / 3) G_{\max }^{3}$.

For finite systems (i.e. molecules, nano-clusters, surfaces), it is also possible to employ plane-wave basis sets. However, care should be taken since large enough vacuum space has to be considered along the finite dimension, to ensure sufficient spacing between neighbouring images, and therefore avoid interactions between these periodic images. The disadvantage of using this technique is that since the electronic density is concentrated in a small fraction of the total volume of the supercell, a very large number of plane-waves would be required (the larger the vacuum space is, the more plane-waves are necessary), thus increasing the computational cost.

More efficient basis sets can be applied to expand the Kohn-Sham orbitals for finite systems. These impose the condition that the wave-functions tend to zero at a distance far enough from the system. Such type of basis sets are mostly implemented in quantum-chemistry codes, and are localized orbitals. Examples include Gaussian basis sets and numeric atom-centered basis sets. The error of these approaches is determined by the number of functions used and the suitability of the choice of these functions for the specific system.

Also, real space basis sets are another alternative, not only for finite systems, but also for periodic crystals. These type of basis functions are sampled on a uniform real-space mesh; therefore, convergence of the results has to be checked against the grid and spacing size of the employed mesh [59].

\section{Pseudopotentials}

For periodic systems it becomes unfeasible to calculate the complete Coulomb potential (external potential term of Eq. 2.56), due to the large number of electrons and basis set size to be considered. Effective potentials (pseudopotentials) attempt to replace the inner core electrons and the nucleus by an inert core that interacts with the valence electrons [59]. 
The concept of a pseudopotential had first been introduced by Enrico Fermi in 1934 [62], in order to describe the scattering of a free neutron by a nucleus. During 1935, Hellman [63] suggested a potential form for potassium, to be able to replace the complex effects of the core electrons with an effective potential. However, it was only during late 50's that the concept of pseudopotential began to be extensively applied for metals and semiconductors, when Phillips and Kleinman [64] generalised an approximation to Hellmann's original pseudopotential form.

Within this approach, the Schrödinger equation will contain a modified effective potential term, representing the core electrons, instead of an explicit Coulombic potential. The wavefunction can thus be written as a sum of a smooth function (pseudo-wavefunction) plus an oscillating function, to maintain orthogonality between the valence and core electrons [59]:

$$
\left|\psi_{v}\right\rangle=\left|\varphi_{v}\right\rangle+\sum_{c} \alpha_{\mathrm{cv}}\left|\psi_{c}\right\rangle
$$

where $\alpha_{\mathrm{cv}}=-\left\langle\psi_{c} \mid \varphi_{v}\right\rangle,\left|\psi_{v}\right\rangle$ and $\left|\psi_{c}\right\rangle$ are the exact solutions of the Schrödinger equation for the valence and the core electrons, respectively, and $\left|\varphi_{v}\right\rangle$ is the pseudo-wavefunction. Since orthogonality ensures that core electrons do not occupy filled valence orbitals, the Pauli exclusion principle will therefore be satisfied.

For the smooth orbital, $\left|\varphi_{v}\right\rangle$, the Schrödinger equation is thus written as

$$
\widehat{H}\left|\varphi_{v}\right\rangle=E_{v}\left|\varphi_{v}\right\rangle+\sum_{c}\left(E_{c}-E_{v}\right)\left|\psi_{c}\right\rangle\left\langle\psi_{c} \mid \varphi_{v}\right\rangle
$$

The most widely employed pseudopotentials for plane-wave electronic structure codes are: norm-conserving, ultrasoft and projector augmented-wave (PAW) pseudopotentials.

Norm-conserving pseudopotentials, were first introduced by Hamann, Sclüter and Chiang [65], and these are built based on ab-initio procedures. The method requires that the pseudo- and all-electron valence eigenstates possess the same energies, amplitudes, and charge densities outside a certain cut-off radius, $r_{l}$ (Fig. 2.1). The integrated charge inside the $r_{l}$ for each wavefunction must agree (norm-conservation), so that the total charge in the core region is correct and the normalized pseudo-orbital equals the true orbital beyond $r_{l}$ [66], enforcing that the pseudo-wavefunction to have the same norm as the true valence wavefunction [59]. The norm-conserving condition can mathematically 


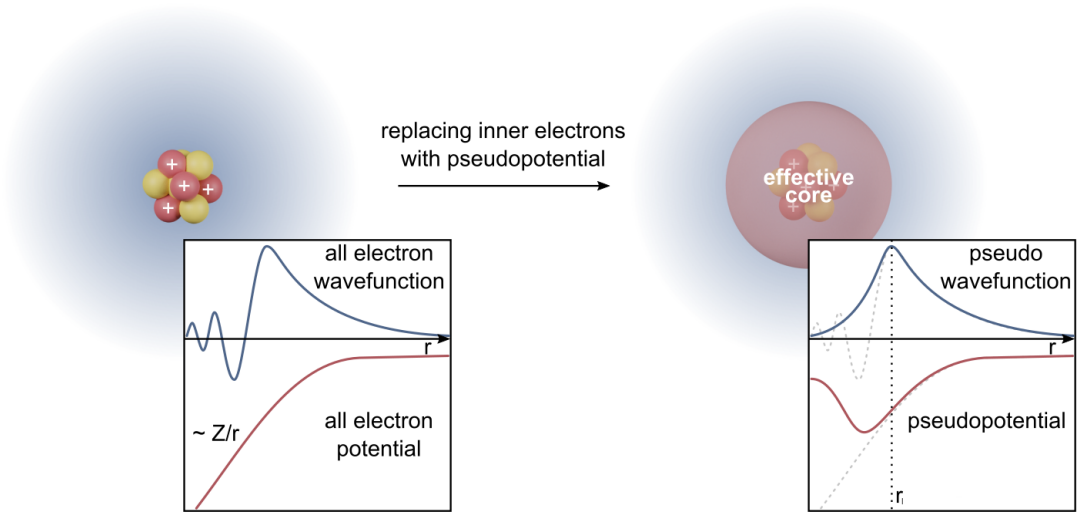

Figure 2.1: Representation of the effective core charge where comparison is made between the all electron wavefunction and the pseudo-wavefuntion (blue), and the Coulomb potential with the pseudopotential (red), up to a certain $r_{l}$ (from Ref. [67]).

be written as:

$$
\begin{aligned}
R_{l}^{\mathrm{PP}}(\mathbf{r})=R_{n l}^{\mathrm{AE}}(\mathbf{r}), & \text { if } r>r_{l} \\
\int_{0}^{r_{l}}\left|R_{l}^{\mathrm{PP}}(\mathbf{r})\right|^{2} r^{2} d r=\int_{0}^{r_{l}}\left|R_{n l}^{\mathrm{AE}}(\mathbf{r})\right|^{2} r^{2} d r, & \text { if } r<r_{l}
\end{aligned}
$$

where $R_{l}(\mathbf{r})$ is the radial part of the wavefunction with angular momentum $l$, and the superscript PP and AE define the pseudo and all-electron wavefunction, respectively.

The cut-off radius influences the accuracy and ability to reproduce realistic electronic and structural properties for different environments (transferability). The quality of the pseudopotential is therefore defined by the choice of the $r_{l}$. The minimum value for the cut-off radius is determined by the location of the outermost nodal surface of the true wavefunction. If $r_{l}$ is close to this minimum, the pseudopotential can reproduce the electronic structure more accurately (hard pseudopotential). On the other hand, if a very large $r_{l}$ is chosen, the pseudopotential will be smooth and almost angular-momentum independent (soft pseudopotential), and these are less accurate to reproduce realistic features in different systems (less transferable). The softer the potential is, the faster the electronic convergence of the plane-wave cut-off must be. The choice of the ideal $r_{l}$ should therefore be a balance between the basis set size and the pseudopotential accuracy.

Ultrasoft pseudopotentials does not enforce norm-conservation. These are constructed to describe a particular atomic environment [59], and therefore are less 
transferable to other systems. The best known approach to develop ultrasoft pseudopotentials was introduced by Vanderbilt [68], and currently it is widely employed, in particular for 3-d transition metals, where a large plane-wave basis-set is frequently necessary to treat strongly-localized orbitals. Blöchl [69] further developed the ultrasoft pseudopotential concept by generalizing the pseudopotential and linear augmented-plane-wave (LAPW) methods into the projector augmented-wave (PAW) method [70]. This method introduces a linear transformation from the pseudo-wavefunction to the all-electron wavefunction (Kohn-Sham single particle wavefunction), operating directly on the full valence and core wavefunctions. Similarly to the LAPW method, PAW can be used to treat first-row and transition-metal elements with affordable basis sets, while providing access to the full wavefunction, and thus to a higher accuracy for a given level of optimization.

\section{Exchange-Correlation Potential}

The xc potential is a functional derivative of the xc energy (Eq. 2.57) with respect to the density. To ensure that the Kohn-Sham formulation remains exact, the xc energy can be defined as

$$
E_{\mathrm{xc}}[n(\mathbf{r})]=T[n(\mathbf{r})]-T_{\mathbf{S}}[n(\mathbf{r})]+E_{\text {ee }}[n(\mathbf{r})]-E_{\text {Hartree }}[n(\mathbf{r})]
$$

where $T[n(\mathbf{r})]$ and $E_{\text {ee }}[n(\mathbf{r})]$ are the exact kinetic and electron-electron interaction energies, respectively, $T_{\mathbf{S}}[n(\mathbf{r})]$ is the Kohn-Sham kinetic energy

$$
T_{\mathbf{S}}[n(\mathbf{r})]=\sum_{i=1} \int \psi_{i}^{*}(\mathbf{r})\left(-\frac{1}{2} \nabla^{2}\right) \psi_{i}(\mathbf{r}) d r
$$

and $E_{\text {Hartree }}$ is the classical Hartree energy of the electrons:

$$
E_{\text {Hartree }}[n(\mathbf{r})]=\frac{1}{2} \iint \frac{n(\mathbf{r}) n\left(\mathbf{r}^{\prime}\right)}{\left|\mathbf{r}-\mathbf{r}^{\prime}\right|} d r d r^{\prime}
$$

The two terms of the xc energy that are not known exactly are the kinetic and interaction terms, which are are grouped into an universal functional. The universal functional reproduces the all-electron kinetic and interaction energies and is independent of the external potential, and is written as:

$$
F_{\mathrm{HK}}[n(\mathbf{r})]=T[n(\mathbf{r})]+E_{\mathrm{ee}}[n(\mathbf{r})],
$$

where HK refers to the the Hohenberg-Kohn theory. Thus, $E_{\mathrm{xc}}$ can be written in terms of HK functional as: 


$$
E_{\mathrm{xc}}[n(\mathbf{r})]=F_{\mathrm{HK}}[n(\mathbf{r})]-\left(T_{\mathbf{S}}[n(\mathbf{r})]+E_{\text {Hartree }}[n(\mathbf{r})]\right) .
$$

The simplest approximation to the xc potential is the Local Density Approximation (LDA) where the potential only depends on the value of the density at point $\mathbf{r}$. LDA has the form [59]:

$$
\begin{aligned}
E_{\mathrm{xc}}^{\mathrm{LDA}}[n] & =\int \varepsilon_{\mathrm{xc}}^{\mathrm{HEG}}[n(\mathbf{r})] d^{3} r \\
v_{\mathrm{xc}}^{\mathrm{LDA}} & =\frac{d}{d n} \varepsilon_{\mathrm{xc}}^{\mathrm{HEG}}[n(\mathbf{r})],
\end{aligned}
$$

where $\varepsilon_{\mathrm{xc}}^{\mathrm{HEG}}(n)$ is the xc energy per unit volume of the homogeneous electron gas (HEG) at constant density $n$.

The Local Spin Density Approximation (LSDA) will have the form

$$
E_{\mathrm{xc}}^{\mathrm{LSDA}}\left[n_{\uparrow}, n_{\downarrow}\right]=\int \varepsilon_{\mathrm{xc}}\left[n_{\uparrow}(\mathbf{r}), n_{\downarrow}(\mathbf{r})\right] d^{3} r
$$

with $\varepsilon_{\mathrm{xc}}\left(n_{\uparrow}(\mathbf{r}), n_{\downarrow}(\mathbf{r})\right)$ being the xc energy per unit volume for an electron gas of uniform spin densities $n_{\uparrow}$ and $n_{\downarrow}$.

The exchange energy of the HEG can easily be calculated from the expression [60]:

$$
E_{\mathrm{x}}^{\mathrm{LDA}}[n]=-\frac{3}{4}\left(\frac{3}{\pi}\right)^{1 / 3} \int n(\mathbf{r})^{4 / 3} d r
$$

On the other hand, the correlation energy of the HEG is obtained through the parametrization of several densities, originally obtained through Monte Carlo methods by Ceperley and Alder [71]. Currently, there exist several parametrized forms for this functional, e.g. PZ81 [72], PW92 [73].

The generalized-gradient approximation (GGA) is a simple extension of the LSDA (Eq. 2.69), and includes the effects of inhomogeneities by including the gradient of the electron density, i.e. $\nabla n$, into $\varepsilon$ (semi-local method)

$$
E_{\mathrm{xc}}^{\mathrm{GGA}}\left[n_{\uparrow}, n_{\downarrow}\right]=\int f\left(n_{\uparrow}, n_{\downarrow}, \nabla n_{\uparrow}, \nabla n_{\downarrow}\right) d^{3} r .
$$

Some results obtained with $\mathrm{L}(\mathrm{S}) \mathrm{DA}$ are found to be in very good agreement with experimental data, including, in some cases, molecular properties such as equilibrium structures, harmonic stretch frequencies, and charge moments 
[61]. Inspite this, the L(S)DA approach tends to fail in predicting other properties. Examples include the incorrect prediction of negative ions to be unstable, underestimation of the fundamental energy band gaps of semiconductors and insulators, overestimation of hydrogen bond lengths, underestimation of volumes.

Similarly, GGA also fails to describe the energy band gaps, which are a crucial physical observable to study, e.g. the impurity levels in doped semiconductors, band alignments, etc. Other common problems and deficiencies of (semi-)local approximations are, i.e. incorrect description of long-range correlation, in particular van-der-Waals (vdW) interactions; incorrect treatment of strongly localized $d-$ and $f$-states [74]. Inspite these failures, GGA can give reasonably accurate results with respect to force constants, charge-densities, energy barriers.

The original GGA functionals had a tendency to overestimate the equilibrium volume, and therefore a revised semi-local functional was subsequently constructed for solids - the Perdew-Burke-Ernzerhof parametrization revised for solids (PBEsol) - which has been successful in improving the description of equilibrium properties such as bond lengths and lattice parameters [75, 76].

Improved xc functionals have been formulated ever since, and due to the large variety of these approximations these have been categorized and grouped into five rungs in a sequence of chemical accuracy, known as the Jacob's ladder of density functional approximations [77]. As the ladder is ascended, the functionals incorporate higher levels of theory with increasingly complex parameters.

\subsubsection{Lattice Dynamics}

When a system is perturbed by an external potential (temperature, pressure, electric fields) it can undergo a phase transition. However, to account for dynamics one must consider the effect of thermal vibrations which generates entropy in the system. The study of the vibrational properties requires accounting for the nuclear motions of the atoms which vibrate with respect to their equilibrium positions. The atomic motions are thus determined by the forces that atoms exert upon each other, from which the dynamical and thermodynamic properties of solids will be obtained.

The normal modes of vibration in a solid can be considered as particle-like and are known as phonons. The energy of a phonon is given by the product of the quantum of action, the Planck's constant, $\hbar$, and the angular frequency, $\omega$. Due 
to fluctuations of the ground-state, characteristic of the harmonic oscillator, the associated zero-point energy of the system is [78]:

$$
E_{0}=\frac{1}{2} \hbar \omega
$$

which corresponds to motions at $T=0 \mathrm{~K}$, known as zero-point vibrations. The mean energy of each vibrational mode, $\nu$, with wave-vector, $\mathbf{q}$, is given by

$$
E(\mathbf{q}, \nu)=\hbar \omega(\mathbf{q}, \nu)\left[\frac{1}{2}+n(\mathbf{q}, \nu)\right]
$$

where $n(\mathbf{q}, \nu)$ is the phonon occupation number for each mode obtained from a Bose-Einstein distribution using the characteristic oscillator frequency $\omega$. The phonon occupation number can be related to temperature, $T$, by

$$
n(\mathbf{q}, \nu)=n(\omega, T)=\left[\frac{1}{\exp \left(\hbar \omega(\mathbf{q}, \nu) / k_{\mathrm{B}} T\right)-1}\right]
$$

where $k_{\mathrm{B}}$ is the Boltzmann constant.

The thermodynamic properties of a solid are directly related to the phonon structure, where the entire set of all possible phonons in the solid are described by the phonon dispersion relations. These combine as the phonon DOS which determines the heat capacity of the solid.

Considering any wave-vector, $\mathbf{q}$, and mode label, $\nu$, the displacement of any atom, $j$, with mass, $m$, in the unit-cell, $l$, will be given by:

$$
\mathbf{u}(j, l)=\frac{1}{\sqrt{N m_{j}}} \sum_{\mathbf{q}, \nu} \mathbf{e}(j, \mathbf{q}, \nu) \exp [i \mathbf{q} \cdot \mathbf{r}(j l)] Q(\mathbf{q}, \nu)
$$

where $\mathbf{r}$ is a vector in real space; $N$ is the number of unit-cells; $\mathbf{e}(\mathbf{q}, \nu)$ is the mode eigenvector (displacement or polarisation vector) which gives the direction of the movement of the atom; and $Q(\mathbf{q}, \nu)$ is the normal mode coordinate that provides the amplitude and the time dependence. The time derivative can be written as:

$$
\dot{Q}(\mathbf{q}, \nu)=-i \omega(\mathbf{q}, \nu) Q(\mathbf{q}, \nu)
$$


The vibrational modes are known as the normal modes of the system, and these are characterized as travelling waves with unique frequency. From Eq. 2.75 one obtains [79]:

$$
Q(\mathbf{q}, \nu)=\frac{1}{\sqrt{N}} \sum_{j, l} \sqrt{m_{j}} \exp [-i \mathbf{q} \cdot \mathbf{r}(j l)] \mathbf{e}^{*}(j, \mathbf{q}, \nu) \cdot \mathbf{u}(j l),
$$

where each normal mode follows the orthogonality relation:

$$
\sum_{j} \mathbf{e}(j, \mathbf{q}, \nu) \cdot \mathbf{e}\left(j,-\mathbf{q}, \nu^{\prime}\right)=\delta_{\nu, \nu^{\prime}}
$$

Within the formalism of normal mode coordinates one can write the Hamiltonian of the system in terms of individual vibrations:

$$
H=\frac{1}{2} \sum_{\mathbf{q}, \nu} \dot{Q}(\mathbf{q}, \nu) \dot{Q}(-\mathbf{q}, \nu)+\frac{1}{2} \sum_{\mathbf{q}, \nu} \omega^{2}(\mathbf{q}, \nu) \dot{Q}(\mathbf{q}, \nu) \dot{Q}(-\mathbf{q}, \nu)
$$

For a unit-cell, there are $3 N$ dispersion branches corresponding to $3 N$ modes of motion. At small wavevectors $(\omega \rightarrow 0$ and $\mathbf{q} \rightarrow 0)$, three modes are related to all atoms moving in phase and these are known as the acoustic modes. All remaining modes (non-zero at $\mathbf{q} \rightarrow 0$ ) are optic modes and refer to atoms moving out-of-phase [78].

By considering the potential energy of a phonon system as a function of the atomic positions, $V\left[\mathbf{u}\left(j_{1}, l_{1}\right), \cdots \mathbf{u}\left(j_{n}, l_{n}\right)\right]$ [79], the potential energy can be expanded in a Taylor series as a function of the atomic displacements from their equilibrium position [80]:

$$
\begin{aligned}
\phi=V_{0} & +\frac{1}{2} \sum_{\substack{j, j^{\prime} \\
l, l^{\prime}}} \frac{\partial^{2} V}{\partial \mathbf{u}_{j, l} \partial \mathbf{u}_{j^{\prime}, l^{\prime}}} \mathbf{u}_{j, l} \mathbf{u}_{j^{\prime}, l^{\prime}}+\cdots \\
& +\frac{1}{n !} \sum_{\substack{j, \cdots, j^{(n)} \\
l, \cdots, l^{(n)}}} \frac{\partial^{n} V}{\partial \mathbf{u}_{j, l} \cdots \partial \mathbf{u}_{j^{(n)}, l^{(n)}}} \mathbf{u}_{j, l} \cdots \mathbf{u}_{j^{(n), l^{(n)}}}
\end{aligned}
$$

where $V_{0}$ is the potential energy with all atoms at rest. The first-order term of the expansion vanishes since it corresponds to a potential minimum. 
The simplest model to study lattice dynamics is the harmonic approximation (HA) in which the Taylor expansion of the potential (Eq. 2.80) is truncated up to second order. The higher order terms in the series expansion of the crystal potential energy correspond to the anharmonic terms. These correspond to the physical effects of anharmonicity in solids such as the thermal volume expansion of solids, the temperature shift of the normal mode frequencies and the thermal resistivity [78].

Phonon frequencies are derived from the restoring force in response to the displacement of ions by a small amplitude from their equilibrium positions. The interatomic force constants (IFCs) can either be computed from perturbation theory (e.g. density-functional perturbation theory, DFPT), or by performing force calculations on a series of symmetry-inequivalent displaced structures. The force/displacement curves are then fitted to a harmonic function. In the latter finite-displacement (direct) method, the Parlinski-Li-Kawazoe supercell approach is commonly employed [81, 82], which allows to capture the long-range contributions to the IFCs between atoms in different crystallographic unit-cells, and required to accurately compute the frequencies of short-wavelength phonon modes [83].

\subsection{High Critical Temperature Superconductors}

\section{Techniques of GFs to Study Superconductors}

The Superconducting (SC) state is characterized by the appearance in the system of a coherent condensate of Cooper pairs related through the GF, of the kind: $\left\langle\hat{a}_{\mathbf{k} \uparrow}^{\dagger} \hat{a}_{\mathbf{k} \downarrow}^{\dagger}\right\rangle$, where $\hat{a}_{\mathbf{k} \uparrow}^{\dagger}$ and $\hat{a}_{\mathbf{k} \downarrow}^{\dagger}$ are the creation operators for electrons with opposite spins. To get the superconductivity equations in compact form, it is convenient to use the technique proposed by Nambu, introducing for the electrons, the spinor operators of two components, the (line) spinor

$$
\hat{\psi}_{\mathbf{k}}^{\dagger}=\left(\begin{array}{cc}
\hat{a}_{\mathbf{k} \uparrow}^{\dagger} & \hat{a}_{-\mathbf{k} \downarrow}
\end{array}\right),
$$

and the (column) spinor:

$$
\hat{\psi}_{\mathbf{k}}=\left(\begin{array}{c}
\hat{a}_{\mathbf{k} \uparrow} \\
\hat{a}_{-\mathbf{k} \downarrow}^{\dagger}
\end{array}\right)
$$


We consider the Pauli matrices:

$$
\hat{\tau}_{1}=\left(\begin{array}{ll}
0 & 1 \\
1 & 0
\end{array}\right), \quad \hat{\tau}_{2}=\left(\begin{array}{cc}
0 & -i \\
i & 0
\end{array}\right), \quad \hat{\tau}_{3}=\left(\begin{array}{cc}
1 & 0 \\
0 & -1
\end{array}\right) .
$$

Now let's consider the normal type functions $\left\langle\left\langle\hat{a}_{\mathbf{k}, \uparrow} \hat{a}_{\mathbf{k}^{\prime}, \uparrow}^{\dagger}\right\rangle\right\rangle$ and the anomalous, $\left\langle\left\langle\hat{a}_{\mathbf{k}, \uparrow} \hat{a}_{-\mathbf{k}^{\prime}, \downarrow}\right\rangle\right\rangle$ then writing them, in a single array,

$$
\hat{G}\left(\mathbf{k}, \mathbf{k}^{\prime}\right)=\left\langle\left\langle\hat{\psi}_{\mathbf{k}} \hat{\psi}_{\mathbf{k}^{\prime}}^{\dagger}\right\rangle\right\rangle
$$

we can write $\hat{G}\left(\mathbf{k}, \mathbf{k}^{\prime}\right)$, explicitly in a matrix form

$$
\hat{G}\left(\mathbf{k}, \mathbf{k}^{\prime}\right)=\left(\begin{array}{cc}
\left\langle\left\langle\hat{a}_{\mathbf{k}, \uparrow} \hat{a}_{\mathbf{k}^{\prime}, \uparrow}^{\dagger}\right\rangle\right\rangle & \left\langle\left\langle\hat{a}_{\mathbf{k}, \uparrow} \hat{a}_{-\mathbf{k}^{\prime}, \downarrow}\right\rangle\right\rangle \\
\left\langle\left\langle\hat{a}_{-\mathbf{k}, \downarrow}^{\dagger} \hat{a}_{\mathbf{k}^{\prime}, \uparrow}\right\rangle\right\rangle & \left\langle\left\langle\hat{a}_{-\mathbf{k}, \downarrow}^{\dagger} \hat{a}_{-\mathbf{k}^{\prime}, \downarrow}\right\rangle\right\rangle
\end{array}\right) .
$$

We see that the diagonal elements of this matrix are the normal GFs for electrons, while the array elements outside the diagonal are anomalous GFs, which are due to the presence in the condensate of Cooper pairs from the system. These two types of GF on superconductivity were introduced by Gor'kov. We define the Fourier transform of the GF matrix in correspondence with the equation

$$
\hat{G}_{\mathbf{k}, \mathbf{k}^{\prime}}(\varepsilon) \equiv\left\langle\left\langle\hat{\psi}_{\mathbf{k}} \mid \hat{\psi}_{\mathbf{k}^{\prime}}^{\dagger}\right\rangle\right\rangle_{\varepsilon}=-i \int_{-\infty}^{0} e^{i(\varepsilon+i 0) t}\left\langle\left\{\psi_{\mathbf{k}^{\prime}}, \psi_{\mathbf{k}^{\prime}}^{\dagger}\right\}\right\rangle d t
$$

where $\hat{G}$ denotes a matrix $2 \times 2$ in the Nambu indices, in which $\langle\ldots\rangle$ is an quantum statistics average, and $\{a(t), b(0)\}$ is the anti-commutator for Heisenberg operators. The matrix elements are the Gor'kov normal and anomalous functions. We distinguish between the Nambu indices (N-indices) and the quasi-momentum indices (M-indices). 
Green's function for the pure crystal in the SC state

The Hamiltonian that describes the SC system in the absence of impurities, can be displayed in compact form as

$$
\hat{H}=\sum_{\mathbf{k}}\left[\hat{\psi}_{\mathbf{k}}^{\dagger}\left(\xi_{\mathbf{k}} \hat{\tau}_{3}-\Delta_{\mathbf{k}} \hat{\tau}_{1}\right) \hat{\psi}_{\mathbf{k}}\right]
$$

where $\hat{\psi}_{\mathbf{k}}$ is the Nambu spinor, $\xi_{\mathbf{k}}$ is the energy of normal quasiparticle with quasimomentum $\mathbf{k}$, and $\tau_{i}$ are the Pauli matrices. The SC parameter $\Delta_{\mathbf{k}}$ satisfies the Bardeen-Cooper-Schrieffer (BCS) gap equation

$$
\Delta_{\mathbf{k}}=-\sum_{\mathbf{k}^{\prime}} V_{S C}\left\langle\hat{a}_{\mathbf{k}^{\prime} \downarrow} \mid \hat{a}_{\mathbf{k}^{\prime} \uparrow}\right\rangle
$$

where the SC coupling constant $V_{S C}$, is attractive for the phonon-mediated electron-electron interaction. The equation of motion of the kind Eq. 2.49, is written for the creation and annihilation operators as follows

$$
\varepsilon\left\langle\left\langle\hat{a}_{\mathbf{k}} \mid \hat{a}_{\mathbf{k}}^{\dagger}\right\rangle\right\rangle=1+\left\langle\left\langle\left[\hat{a}_{\mathbf{k}}, \hat{H}\right] \mid \hat{a}_{\mathbf{k}}^{\dagger}\right\rangle\right\rangle
$$

we can write the equation Eq: 2.84 as

$$
\varepsilon\left\langle\left\langle\hat{a}_{\mathbf{k}_{\uparrow}} \mid \hat{a}_{\mathbf{k}^{\prime} \downarrow}^{\dagger}\right\rangle\right\rangle=1+\xi_{\mathbf{k}}\left\langle\left\langle\hat{a}_{\mathbf{k} \uparrow} \mid \hat{a}_{\mathbf{k}^{\prime} \downarrow}^{\dagger}\right\rangle\right\rangle-\Delta_{\mathbf{k}}\left\langle\left\langle\hat{a}_{-\mathbf{k} \downarrow}^{\dagger} \mid \hat{a}_{\mathbf{k}^{\prime} \downarrow}^{\dagger}\right\rangle\right\rangle .
$$

For the GFs in the right part of Eq. 2.85, we can rewrite the equations of motion. So the next equation to consider, for the next element in Nambu column matrix is:

$$
\varepsilon\left\langle\left\langle\hat{a}_{-\mathbf{k} \downarrow}^{\dagger} \mid \hat{a}_{\mathbf{k}^{\prime} \downarrow}^{\dagger}\right\rangle\right\rangle=-\xi_{\mathbf{k}}\left\langle\left\langle\hat{a}_{-\mathbf{k} \downarrow}^{\dagger} \mid \hat{a}_{\mathbf{k}^{\prime} \downarrow}^{\dagger}\right\rangle\right\rangle-\Delta_{\mathbf{k}}\left\langle\left\langle\hat{a}_{\mathbf{k}_{\uparrow} \uparrow} \mid \hat{a}_{\mathbf{k}^{\prime} \downarrow}^{\dagger}\right\rangle\right\rangle,
$$

We can now present Eqs. 2.85 and 2.86 in the form:

$$
\begin{aligned}
\left(\varepsilon-\xi_{\mathbf{k}}\right)\left\langle\left\langle\hat{a}_{\mathbf{k} \uparrow} \mid \hat{a}_{\mathbf{k}^{\prime} \downarrow}^{\dagger}\right\rangle\right\rangle & =1-\Delta_{\mathbf{k}}\left\langle\left\langle\hat{a}_{-\mathbf{k} \downarrow}^{\dagger} \mid \hat{a}_{\mathbf{k}^{\prime} \downarrow}^{\dagger}\right\rangle\right\rangle, \\
\left(\varepsilon+\xi_{\mathbf{k}}\right)\left\langle\left\langle\hat{a}_{-\mathbf{k} \downarrow}^{\dagger} \mid \hat{a}_{\mathbf{k}^{\prime} \downarrow}^{\dagger}\right\rangle\right\rangle & =-\Delta_{\mathbf{k}}\left\langle\left\langle\hat{a}_{\mathbf{k}_{\uparrow} \uparrow} \hat{a}_{\mathbf{k}^{\prime} \downarrow}^{\dagger}\right\rangle\right\rangle .
\end{aligned}
$$

Multiplying the first expression of 2.87 for $\left(\varepsilon+\xi_{\mathbf{k}}\right)$ becomes: 


$$
\left(\varepsilon^{2}-\xi_{\mathbf{k}}^{2}\right)\left\langle\left\langle\hat{a}_{\mathbf{k} \uparrow} \mid \hat{a}_{\mathbf{k}^{\prime} \downarrow}^{\dagger}\right\rangle\right\rangle=\left(\varepsilon+\xi_{\mathbf{k}}\right)-\Delta_{\mathbf{k}}\left(\varepsilon+\xi_{\mathbf{k}}\right)\left\langle\left\langle\hat{a}_{-\mathbf{k} \downarrow}^{\dagger} \mid \hat{a}_{\mathbf{k}^{\prime} \downarrow}^{\dagger}\right\rangle\right\rangle,
$$

and multiplying the second term by $-\Delta_{\mathrm{k}}$ one gets:

$$
-\Delta_{\mathbf{k}}\left(\varepsilon+\xi_{\mathbf{k}}\right)\left\langle\left\langle\hat{a}_{-\mathbf{k} \downarrow}^{\dagger} \mid \hat{a}_{\mathbf{k}^{\prime} \downarrow}^{\dagger}\right\rangle\right\rangle=-\Delta_{\mathbf{k}}^{2}\left\langle\left\langle\hat{a}_{\mathbf{k}_{\uparrow}} \mid \hat{a}_{\mathbf{k}^{\prime} \downarrow}^{\dagger}\right\rangle\right\rangle .
$$

Therefore, by replacing Eq. 2.88 on Eq. 2.89 we get:

$$
\left(\varepsilon^{2}-\xi_{\mathbf{k}}^{2}\right)\left\langle\left\langle\hat{a}_{\mathbf{k} \uparrow} \mid \hat{a}_{\mathbf{k}^{\prime} \downarrow}^{\dagger}\right\rangle\right\rangle=\varepsilon+\xi_{\mathbf{k}}-\Delta_{\mathbf{k}}^{2}\left\langle\left\langle\hat{a}_{-\mathbf{k} \downarrow} \mid \hat{a}_{\mathbf{k}^{\prime} \downarrow}^{\dagger}\right\rangle\right\rangle,
$$

so the explicit results for the above considered GFs:

$$
\begin{aligned}
\left\langle\left\langle\hat{a}_{\mathbf{k} \uparrow} \mid \hat{a}_{\mathbf{k}^{\prime} \downarrow}^{\dagger}\right\rangle\right\rangle & =\frac{\varepsilon+\xi_{\mathbf{k}}}{\varepsilon^{2}-\xi_{\mathbf{k}}^{2}-\Delta_{\mathbf{k}}^{2}}, \\
\left\langle\left\langle\hat{a}_{-\mathbf{k} \downarrow}^{\dagger} \mid \hat{a}_{\mathbf{k}^{\prime} \downarrow}^{\dagger}\right\rangle\right\rangle & =\frac{-\Delta_{\mathbf{k}}}{\varepsilon^{2}-\xi_{\mathbf{k}}^{2}-\Delta_{\mathbf{k}}^{2}} .
\end{aligned}
$$

Proceeding similarly, one can calculate the elements of the second column of the Nambu matrix Eq. 2.82, yielding the corresponding expressions for $\left\langle\left\langle\hat{a}_{\mathbf{k} \uparrow} \mid \hat{a}_{-\mathbf{k}^{\prime} \downarrow}\right\rangle\right\rangle$ and $\left\langle\left\langle\hat{a}_{-\mathbf{k} \downarrow}^{\dagger} \mid \hat{a}_{-\mathbf{k}^{\prime} \downarrow}^{\dagger}\right\rangle\right\rangle$. With these expressions and the equation Eq. 2.82 we get the matrix form of GF for the SC uniform state,

$$
\hat{G}_{\mathbf{k}}^{0}=\left(\varepsilon-\Delta_{\mathbf{k}} \hat{\tau}_{1}-\xi_{\mathbf{k}} \hat{\tau}_{3}\right)^{-1}
$$

DOS for the SC Pure Crystal

By performing the inversion of matrices, Eq. 2.91, we have

$$
\hat{G}_{\mathbf{k}}^{0}=\frac{1}{\varepsilon^{2}-\xi_{\mathbf{k}}^{2}-\Delta_{\mathbf{k}}^{2}}\left[\begin{array}{cc}
\varepsilon+\xi_{\mathrm{k}} & \Delta_{\mathbf{k}} \\
\Delta_{\mathrm{k}} & \varepsilon-\xi_{\mathrm{k}}
\end{array}\right]
$$

then the DOS is given by:

$$
\rho(\varepsilon)=\operatorname{Im} \sum_{\mathbf{k}} \operatorname{Tr} \hat{G}_{\mathbf{k}}^{0}=\frac{1}{2 \pi N} \operatorname{Im} \sum_{\mathbf{k}} \frac{2 \varepsilon}{\varepsilon^{2}-\xi_{\mathbf{k}}^{2}-\Delta_{\mathbf{k}}^{2}},
$$


By applying the integration rule for delta functions [84]:

$$
\int d x \delta\left(g(x) f(x)=\sum_{i} \frac{f\left(x_{i}\right)}{\left|g^{\prime}\left(x_{i}\right)\right|}\right.
$$

where $x_{i}$ are the roots of the equation $g\left(x_{i}\right)=0$ that lie within the integration area.

By applying the rule given in Eq. 2.93 to Eq. 2.92 we obtain:

$$
\begin{aligned}
\rho(\varepsilon) & =2 \varepsilon \frac{a^{2}}{(2 \pi)^{2}} \int \delta\left(\varepsilon^{2}-\xi_{\mathbf{k}}^{2}-\Delta_{\mathbf{k}}^{2}\right) k d k d \varphi \\
& =2 \varepsilon \frac{a^{2}}{(2 \pi)^{2}} \int k \delta\left(\varepsilon^{2}-\hbar^{2} v_{F}^{2}\left|k-k_{F}\right|^{2}-\Delta_{\mathbf{k}}^{2}\right) d k d \varphi .
\end{aligned}
$$

for which the relevant roots are

$$
k=k_{F} \mp \sqrt{\frac{\varepsilon^{2}-\Delta^{2}}{\hbar v_{F}}} .
$$

The particular result is therefore dependant on the symmetry of the order parameter $\Delta_{\mathbf{k}}$. Thus, for $s$-wave, $\Delta_{\mathbf{k}}=\Delta$, the DOS shows the similar characteristic of the BCS theory:

$$
\rho(\varepsilon)=\frac{2 \varepsilon}{\pi^{2} W} \int_{0}^{2 \pi} \frac{d \varphi}{\sqrt{\varepsilon^{2}-\Delta^{2}}}=\rho_{0} \frac{\varepsilon}{\sqrt{\varepsilon^{2}-\Delta^{2}}} .
$$

The case more relevant to HTSC with $d$-wave symmetry, it is associated with the order parameter in the form: $\Delta_{\mathbf{k}}=\Delta \cos 2 \varphi$ (corresponding to the circular Fermi surface approach). The last expression in Eq.2.94 is written in terms of elliptic integrals as:

$$
\rho(\varepsilon)=\frac{2 \varepsilon}{\pi^{2} W} \int_{0}^{2 \pi} \frac{d \varphi}{\sqrt{\varepsilon^{2}-\Delta^{2} \cos ^{2} 2 \varphi}}=\frac{2}{\sqrt{\Delta^{2}-\varepsilon^{2}}} \mathrm{~K}\left(\frac{\varepsilon^{2}}{\Delta^{2}-\varepsilon^{2}}\right),
$$


where, $K(x)$ is the elliptic integral of the first kind. The singularity at point $\varepsilon=\Delta$ is similar to the results obtained in experimental measurements from tunnel spectroscopy.

\subsubsection{Impurities Effects and the Self-Energy Matrix}

The tight-binding approach comes from the idea that all the charge carriers occupy the atomic states located on the lattice sites with the same energy $\varepsilon_{a}$, and that these can perform jumps between close neighbors sites with a certain transition amplitude $t \ll \varepsilon_{a}$. The corresponding Hamiltonian is expressed in terms of local operators $\hat{a}_{\mathbf{n}, \sigma}, \hat{a}_{\mathbf{n}, \sigma}^{\dagger}$ as:

$$
\hat{H}_{0}=\sum_{\mathbf{n}, \sigma}\left(\varepsilon_{a} \hat{a}_{\mathbf{n}, \sigma}^{\dagger} \hat{a}_{\mathbf{n}, \sigma}+t \sum_{\delta} \hat{a}_{\mathbf{n}+\delta}^{\dagger} \hat{a}_{\mathbf{n}}\right),
$$

where the vectors $\boldsymbol{\delta}$ link the site $\mathbf{n}$ with the sites which are its close neighbors on the lattice and $t$ is the jump amplitude for these sites. Using the translational symmetry, the band energy, for the tight binding Hamiltonian approach is written,

$$
\hat{H}_{0}=\sum_{\mathbf{n}}\left(\varepsilon_{a} \hat{a}_{\mathbf{n}}^{\dagger} \hat{a}_{\mathbf{n}}+t \sum_{\delta} \hat{a}_{\mathbf{n}}^{\dagger} \hat{a}_{\mathbf{n}+\delta}\right)=\sum_{\mathbf{k}} \varepsilon_{\mathbf{k}} \hat{a}_{\mathbf{k}}^{\dagger} \hat{a}_{\mathbf{k}},
$$

where we have used the Fourier transform for operators in the form:

$$
\hat{a}_{\mathbf{k}, \sigma}=\frac{1}{\sqrt{N}} \sum_{\mathbf{k}} e^{-i \mathbf{k} \cdot \mathbf{n}} \hat{a}_{\mathbf{n}, \sigma} .
$$

So the Hamiltonian (2.95) can be diagonalized, and the dispersion law, that is given by the general expression: $\varepsilon_{\mathbf{k}}=\varepsilon_{a}+t \sum_{\delta} e^{i \mathbf{k} \cdot \delta}$,

in the particular case of the 2D square lattice, where the vectors $\boldsymbol{\delta}$ are directed along the axis $a$, got the form $\varepsilon_{\mathbf{k}}=4 t-2 t\left(\cos a k_{x}+\cos a k_{y}\right)$.

For the more general case, in which it is considered the presence of impurities, with impurity potential $V$, which occupy localized states in the sites p. We can further express the Hamiltonian of this system in terms of plane wave operators $\hat{a}_{\mathbf{k}}$ by writing: 


$$
\hat{H}=\sum_{\mathbf{k}} \varepsilon_{\mathbf{k}} \hat{a}_{\mathbf{k}}^{\dagger} \hat{a}_{\mathbf{k}}+\frac{V}{N} \sum_{\mathbf{p}, \mathbf{k}, \mathbf{k}^{\prime}} e^{i\left(\mathbf{k}-\mathbf{k}^{\prime}\right) \cdot \mathbf{p}} \hat{a}_{\mathbf{k}^{\prime}}^{\dagger} \hat{a}_{\mathbf{k}}
$$

\section{Green Functions and Motion Equation}

Considerations on the effects of introducing impurities in a physical system allows one to write the equation of motion Eq. 2.52 in terms of GF,

$$
\varepsilon \hat{G}_{\mathbf{k}}^{0}(\varepsilon)=\left\langle\left\{\hat{a}_{\mathbf{k}}^{\dagger}, \hat{a}_{\mathbf{k}}\right\}\right\rangle+\left\langle\left\langle\left[\hat{a}_{\mathbf{k}}, \hat{H}\right] \mid \hat{a}_{\mathbf{k}}^{\dagger}\right\rangle\right\rangle
$$

being the Hamiltonian written in the form: $\hat{H}=\hat{H}_{0}+\hat{H}_{i m p}$, where $\hat{H}_{i m p}$ is the term that describes the impurities, we can re-write

$$
\hat{G}_{\mathbf{k}}=\left\langle\left\langle\hat{a}_{\mathbf{k}} \mid \hat{a}_{\mathbf{k}}^{\dagger}\right\rangle\right\rangle=1+\left\langle\left\langle\left[\hat{a}_{\mathbf{k}}, \hat{H}_{i m p}\right] \mid \hat{a}_{\mathbf{k}}^{\dagger}\right\rangle\right\rangle
$$

By employing the Fourier transforms for the operators,

$$
\hat{a}_{\mathbf{p}, \sigma}=\frac{1}{\sqrt{N}} \sum_{\mathbf{k}} e^{-i \mathbf{k} \cdot \mathbf{p}} \hat{a}_{\mathbf{k}, \sigma}
$$

and the commutator:

$$
\left[\hat{a}_{\mathbf{k}}, \hat{H}_{i m p}\right]=\frac{V}{N} \sum_{\mathbf{p}, \mathbf{k}^{\prime}} e^{-i\left(\mathbf{k}-\mathbf{k}^{\prime}\right) \cdot \mathbf{p}} \hat{a}_{\mathbf{k}^{\prime}}
$$

it is possible to study the equation of motion (the terms of multiple diffusions). In particular, the above considerations will allow to re-write Eq. 2.97 in the following manner:

$$
\begin{gathered}
\left(\varepsilon-\varepsilon_{\mathbf{k}}\right)\left\langle\left\langle\hat{a}_{\mathbf{k}} \mid \hat{a}_{\mathbf{k}}^{\dagger}\right\rangle\right\rangle=1+\frac{V}{N} \sum_{\mathbf{p}, \mathbf{k}^{\prime}} e^{-i\left(\mathbf{k}-\mathbf{k}^{\prime}\right) \cdot \mathbf{p}}\left\langle\left\langle\hat{a}_{\mathbf{k}^{\prime}} \mid \hat{a}_{\mathbf{k}}^{\dagger}\right\rangle\right\rangle \\
=1+\frac{V}{N} \sum_{\mathbf{p}}\left\langle\left\langle\hat{a}_{\mathbf{k}} \mid \hat{a}_{\mathbf{k}}^{\dagger}\right\rangle\right\rangle+\frac{V}{N} \sum_{\mathbf{p}, \mathbf{k}^{\prime} \neq \mathbf{k}} e^{-i\left(\mathbf{k}-\mathbf{k}^{\prime}\right) \cdot \mathbf{p}}\left\langle\left\langle\hat{a}_{\mathbf{k}^{\prime}} \mid \hat{a}_{\mathbf{k}}^{\dagger}\right\rangle\right\rangle
\end{gathered}
$$

where $(V / N) \sum_{\mathbf{p}}=c V$. In this way, the equations of motion for the function $\left\langle\left\langle\hat{a}_{\mathbf{k}^{\prime}} \mid \hat{a}_{\mathbf{k}}^{\dagger}\right\rangle\right\rangle_{\mathbf{k}^{\prime} \neq \mathbf{k}}$, which appears in the above expression, gives: 


$$
\begin{aligned}
\left(\varepsilon-\varepsilon_{\mathbf{k}^{\prime}}\right)\left\langle\left\langle\hat{a}_{\mathbf{k}^{\prime}} \mid \hat{a}_{\mathbf{k}}^{\dagger}\right\rangle\right\rangle & =\frac{V}{N} \sum_{\mathbf{p}^{\prime}, \mathbf{k}^{\prime \prime}} e^{-i\left(\mathbf{k}^{\prime}-\mathbf{k}^{\prime \prime}\right) \cdot \mathbf{p}^{\prime \prime}}\left\langle\left\langle\hat{a}_{\mathbf{k}^{\prime \prime}} \mid \hat{a}_{\mathbf{k}}^{\dagger}\right\rangle\right\rangle \\
& =\frac{V}{N} \sum_{\mathbf{p}^{\prime}} e^{-i\left(\mathbf{k}-\mathbf{k}^{\prime}\right) \cdot \mathbf{p}}\left\langle\left\langle\hat{a}_{\mathbf{k}} \mid \hat{a}_{\mathbf{k}}^{\dagger}\right\rangle\right\rangle+\ldots
\end{aligned}
$$

where we have ignored the terms in $\mathbf{k}^{\prime \prime} \neq \mathbf{k}^{\prime}$. So placing the Eq. 2.99 in Eq. 2.98 we obtain:

$$
\left(\varepsilon-\varepsilon_{\mathbf{k}}-c V-\frac{\hat{V}}{N} \sum_{\mathbf{p}^{\prime}, \mathbf{k}^{\prime} \neq \mathbf{k}} e^{-i\left(\mathbf{k}-\mathbf{k}^{\prime}\right) \cdot \mathbf{p}} \frac{V}{N} \frac{e^{-i\left(\mathbf{k}-\mathbf{k}^{\prime}\right) \cdot \mathbf{p}}}{\varepsilon-\varepsilon_{\mathbf{k}^{\prime}}}\right)\left\langle\left\langle\hat{a}_{\mathbf{k}} \mid \hat{a}_{\mathbf{k}}^{\dagger}\right\rangle\right\rangle=1+\ldots
$$

which can be re-written as:

$$
\left\langle\left\langle\hat{a}_{\mathbf{k}} \mid \hat{a}_{\mathbf{k}}^{\dagger}\right\rangle\right\rangle=\left(\varepsilon-\varepsilon_{\mathbf{k}}-c V-\frac{c V^{2}}{N} \sum_{\mathbf{k}^{\prime} \neq \mathbf{k}} \frac{1}{\varepsilon-\varepsilon_{\mathbf{k}^{\prime}}}+\ldots\right)^{-1},
$$

we define $G(\varepsilon)=(1 / N) \sum_{\mathbf{k}} G_{\mathbf{k}}(\varepsilon)$, and present the GF for describing the impure systems,

$$
\left\langle\left\langle\hat{a}_{\mathbf{k}} \mid \hat{a}_{\mathbf{k}}^{\dagger}\right\rangle\right\rangle \approx\left(\varepsilon-\varepsilon_{\mathbf{k}}-\frac{c V}{1-V G(\varepsilon)}\right)^{-1}=\frac{1}{\varepsilon-\varepsilon_{\mathbf{k}}-\Sigma_{\mathbf{k}}},
$$

where the sum $c V+c V^{2} G(\varepsilon)+c V^{3} G^{2}(\varepsilon)+c V^{4} G^{3}(\varepsilon)+\ldots$ is expressed by a geometric series $c V /(1-V G(\varepsilon))$. The representation $\Sigma_{\mathbf{k}}$ refers to the selfenergy matrix, which in the most general case, we can write as

$$
\Sigma_{\mathbf{k}}=\frac{c V}{1-\hat{V} \hat{G}(\varepsilon)}\left(1-c \sum_{\mathbf{n} \neq 0} \frac{A_{0 \mathbf{n}} e^{-i \mathbf{k} \cdot \mathbf{n}}+A_{0 \mathbf{n}} A_{\mathbf{n} \mathbf{0}}}{1-A_{0 \mathbf{n}} A_{\mathbf{n} 0}}+\ldots\right),
$$

where the matrices $A_{0 \mathrm{n}}$ relates to indirect interaction between the scattering sites $\mathbf{0}$ and $\mathbf{n}$ are:

$$
A_{0 \mathbf{n}}=V \sum_{\mathbf{k}^{\prime} \neq \mathbf{k}} \frac{e^{i \mathbf{k}^{\prime} \cdot \mathbf{n}}}{\varepsilon-\varepsilon_{\mathbf{k}}}[1-V \hat{G}(\varepsilon)]^{-1} .
$$


These expansions refers to indirect interactions between the impurity centers, the self-energy $\Sigma_{\mathbf{k}}$ where the first term T-matrix, is followed by a group series in increasing numbers of interacting impurity centers. Alike the classical UrsellMayer group expansions [85].

Impurity Perturbations and Group Expansions (GE)

To study the effect of the perturbations introduced by impurities in a material we use the model of Lifshitz whose potential $V_{\mathrm{i}}$ is point type in random sites $\mathbf{p}$ and with concentration $c \ll 1,[22]$. The Hamiltonian in the presence of impurities is written as $H+H^{\prime}$, where $H^{\prime}$ refers the perturbation matrix $\widehat{V} \propto V_{\mathrm{i}}$,

$$
H^{\prime}=\frac{1}{N} \sum_{\mathbf{p}, \mathbf{k}, \mathbf{k}^{\prime}} \mathrm{e}^{i\left(\mathbf{k}-\mathbf{k}^{\prime}\right) \mathbf{p}} \psi_{\mathbf{k}^{\prime}}^{\dagger} \widehat{V} \psi_{\mathbf{k}}
$$

The equation of motion in the presence of impurities is written as:

$$
\widehat{G}_{\mathbf{k}, \mathbf{k}^{\prime}}=\delta_{\mathbf{k}, \mathbf{k}^{\prime}} \widehat{G}_{\mathbf{k}}^{0}-\frac{1}{N} \sum_{\mathbf{p}, \mathbf{k}^{\prime \prime}} \mathrm{e}^{i\left(\mathbf{k}-\mathbf{k}^{\prime \prime}\right) \mathbf{p}} \widehat{G}_{\mathbf{k}}^{0} \widehat{V} \widehat{G}_{\mathbf{k}^{\prime \prime}, \mathbf{k}^{\prime}}
$$

We can choose several routines to approximate these to an infinite chain of equations of the type $\widehat{G}_{\mathbf{k}^{\prime \prime}, \mathbf{k}^{\prime}}$, in which Eq. 2.103 describes the scattering processes. In particular, analyzing the consecutive interactions of this equation allows for the GE routine to be fully renormalized, proceeding to the systematic separation of all interaction terms that figured in previous interactions. So we separate the function $G_{\mathbf{k}}$ from those that include the scattering terms, $\widehat{G}_{\mathbf{k}^{\prime}, \mathbf{k}}, \mathbf{k}^{\prime} \neq \mathbf{k}$ :

$$
\begin{aligned}
\widehat{G}_{\mathbf{k}} & =\widehat{G}_{\mathbf{k}}^{0}+\frac{1}{N} \sum_{\mathbf{k}^{\prime}, \mathbf{p}} \mathrm{e}^{i\left(\mathbf{k}-\mathbf{k}^{\prime}\right) \cdot \mathbf{p}} \widehat{G}_{\mathbf{k}}^{0} \widehat{V} \widehat{G}_{\mathbf{k}^{\prime}, \mathbf{k}} \\
& =\widehat{G}_{\mathbf{k}}^{0}+c \widehat{G}_{\mathbf{k}}^{0} \widehat{V} G_{\mathbf{k}}+\frac{1}{N} \sum_{\mathbf{k}^{\prime} \neq \mathbf{k}, \mathbf{p}} \mathrm{e}^{i\left(\mathbf{k}-\mathbf{k}^{\prime}\right) \cdot \mathbf{p}} \widehat{G}_{\mathbf{k}}^{0} \widehat{V} \widehat{G}_{\mathbf{k}^{\prime}, \mathbf{k}}
\end{aligned}
$$

Then for each $\widehat{G}_{\mathbf{k}^{\prime}, \mathbf{k}}, \mathbf{k}^{\prime} \neq \mathbf{k}$ we write again Eq. 2.103 and, in the right hand side, one can find the isolated scatter terms of $\widehat{G}_{\mathbf{k}}$ and $\widehat{G}_{\mathbf{k}^{\prime}, \mathbf{k}}$ : 


$$
\begin{aligned}
\widehat{G}_{\mathbf{k}^{\prime}, \mathbf{k}} & =\frac{1}{N} \sum_{\mathbf{k}^{\prime \prime}, \mathbf{p}^{\prime}} \mathrm{e}^{i\left(\mathbf{k}^{\prime}-\mathbf{k}^{\prime \prime}\right) \cdot \mathbf{p}} \widehat{G}_{\mathbf{k}^{\prime}}^{0} \widehat{V} \widehat{G}_{\mathbf{k}^{\prime \prime}, \mathbf{k}} \\
& =c \widehat{G}_{\mathbf{k}^{\prime}}^{0} \widehat{V} \widehat{G}_{\mathbf{k}^{\prime}, \mathbf{k}}+\frac{1}{N} \mathrm{e}^{i\left(\mathbf{k}^{\prime}-\mathbf{k}\right) \cdot \mathbf{p}} \widehat{G}_{\mathbf{k}^{\prime}}^{0} \widehat{V} \widehat{G}_{\mathbf{k}} \\
& +\frac{1}{N} \sum_{\mathbf{p}^{\prime} \neq \mathbf{p}} \mathrm{e}^{i\left(\mathbf{k}^{\prime}-\mathbf{k}\right) \cdot \mathbf{p}^{\prime}} \widehat{G}_{\mathbf{k}^{\prime}}^{0} \widehat{V} \widehat{G}_{\mathbf{k}} \\
& +\frac{1}{N} \sum_{\mathbf{k}^{\prime \prime} \neq \mathbf{k}, \mathbf{k}^{\prime} ; \mathbf{p}^{\prime}} \mathrm{e}^{i\left(\mathbf{k}^{\prime}-\mathbf{k}^{\prime \prime}\right) \cdot \mathbf{p}^{\prime}} \widehat{G}_{\mathbf{k}^{\prime}}^{0} \widehat{V} \widehat{G}_{\mathbf{k}^{\prime \prime}, \mathbf{k}}
\end{aligned}
$$

It must be noted that among the terms with $\widehat{G}_{\mathbf{k}}$, the term with $\mathbf{p}^{\prime}=\mathbf{p}$ (second on the right side of Eq. 2.105), contains the phase factor $\mathrm{e}^{i\left(\mathbf{k}^{\prime}-\mathbf{k}\right) \cdot \mathbf{p}}$. Therefore, this expression is consistent with the previously presented in the last sum of Eq. 2.104. Consequently, this term is explicitly separated from the incoherent terms, $\propto \mathrm{e}^{i\left(\mathbf{k}^{\prime}-\mathbf{k}\right) \cdot \mathbf{p}^{\prime}}$, with $\mathbf{p}^{\prime} \neq \mathbf{p}$.

Carrying on with this sequence, we have collected the terms whose initial function $\widehat{G}_{\mathbf{k}}$ results in:

1. all multiple scattering on the same site $\mathbf{p}$,

2. processes on the same pair sites $\mathbf{p}$ and $\mathbf{p}^{\prime} \neq \mathbf{p}$, and so on.

Then, the sum in $\mathbf{p}$ of the terms 1)- result in the first term of GE:

$$
\widehat{T}=\widehat{V}(1-\widehat{G} \widehat{V})^{-1},
$$

and if we ignore the processes in the impurity clusters, this term identifies with the self-consistent $T$-matrix [86]. The second term of GE is obtained by the sum of terms 2), with $\mathbf{p}, \mathbf{p}^{\prime} \neq \mathbf{p}$, which contain interaction matrices [87] $\widehat{A}_{\mathbf{p}^{\prime}, \mathbf{p}}=N^{-1} \sum_{\mathbf{k}^{\prime}} \mathrm{e}^{i \mathbf{k}^{\prime} \cdot \mathbf{p}} \widehat{G}_{\mathbf{k}^{\prime}} \widehat{T}$ generated from the GF of multiple diffusions $\widehat{G}_{\mathbf{k}^{\prime}, \mathbf{k}}$, $\mathbf{k}^{\prime} \neq \mathbf{k} \ldots$

In summary, we obtain a fully normalized representation for M-diagonal GF

$$
\widehat{G}_{\mathbf{k}}=\widehat{G}_{\mathbf{k}, \mathbf{k}}=\left[\left(\widehat{G}_{\mathbf{k}}^{0}\right)^{-1}-\widehat{\Sigma}_{\mathbf{k}}\right]^{-1}
$$


where the renormalized matrix self energy is represented by GE

$$
\widehat{\Sigma}_{\mathbf{k}}=c \widehat{T}\left(1-c \widehat{A}_{0}-c \widehat{A}_{0}^{2}+c \widehat{B}_{\mathbf{k}}+\cdots\right)
$$

with the pair term

$$
\widehat{B}_{\mathbf{k}}=\sum_{\mathbf{n} \neq 0}\left(\widehat{A}_{\mathbf{n}}^{3} \mathrm{e}^{-i \mathbf{k n}}+\widehat{A}_{\mathbf{n}}^{4}\right)\left(1-\widehat{A}_{\mathbf{n}}^{2}\right)^{-1}
$$

The term that follows the unity in Eq. 2.101, describes the contributions from all possible clusters of two impurities, with the remaining terms referring to contributions of clusters with three or more impurities. This allows us to describe a hierarchical structure for localized states in a crystal with impurities [88].

\title{
2.6 Overview of Low-Dimensional Physics in 2D Graphene
}

\author{
Niels Bohr: "What are you working on Mr. Dirac?" \\ Paul Dirac: "I'm trying to take the square root of something"
}

To describe the electronic properties of monolayer graphene, we compute the spectrum of the material by employing the Tight-Binding theory. By performing the calculation in the low energy limit we will obtain the linear dispersion, which is one of the characteristics of Dirac massless relativistic particles.

We also focus on disordered systems which is obtained by applying perturbations (impurities). The DOS of the intrinsic graphene system will be calculated by employing the GF technique. The DOS is a fundamental physical quantity which aids in the interpretation of several experimental data for disordered systems.

The effect of impurities will be calculated by employing the simple and selfconsistent T-matrix method. For the considered case of finite impurity concentration, the self-consistent method is more reliable. However, with the exception of the 'spurious' effect obtained for the vacancy (DOS divergence for 
$\varepsilon=0$ ), that persists even with the self-consistent technique, all other observables treated within this formalism will be in accordance with the experimental data and other theoretical approximations.

\subsubsection{Graphene Spectrum}

Graphene is a carbon allotrope constructed by an hexagonal lattice due to the $\mathrm{sp}_{2}$ hybridization between the $s$ orbital and two $p$ orbitals. The $\mathrm{sp}_{2}$ orbital leads to sigma bonds, while the orbital that does not enter the hybridization process, $p_{z}$, will form a $\pi$ bond with its neighbors, which is the origin of the conducting properties of the material. Therefore a valence electron per atomic site is considered. The $\pi$-type bonds can thus be associated with the conduction band and the $\sigma$-type bonds to the valence band.

The hexagonal crystalline structure of graphene is well known in condensed matter physics as being composed of two triangular sub-lattices (mathematically labeled as 'non-equivalent', $A$ and $B$ ) each consisting of two atoms in the unit-cell (with atom $a \in A$ and $b \in B$ ), in order to satisfy the transational properties and symmetry of a Bravais lattice Fig 2.2. As a consequence, two inequivalent points in the reciprocal lattice are identified, $K$ and $K^{\prime}$.

The characteristics of the hexagonal lattice, are defined by the lattice vectors:

$a_{1}=\frac{a}{2}(3, \sqrt{3}), \quad a_{2}=\frac{a}{2}(3,-\sqrt{3})$, with $a \approx 1.42 \AA$. and three next-neighbors (real space)

$$
\delta_{1}=\frac{a}{2}(1, \sqrt{3}), \delta_{2}=\frac{a}{2}(1,-\sqrt{3}), \delta_{3}=-a(1,0) .
$$

The reciprocal lattice vectors obtained by the relation $b_{i} \cdot a_{j}=2 \pi \delta_{i j}$ are:

$$
b_{1}=\frac{2 \pi}{3 a}(1, \sqrt{3}), \quad b_{2}=\frac{2 \pi}{3 a}(1,-\sqrt{3})
$$

\section{'Tight-Binding' Hamiltonian}

To obtain the tight-binding Hamiltonian, one considers electronic transitions which occur only between next-neighbors

$$
H=t \sum_{n, \delta}\left(a_{n}^{\dagger} b_{n+\delta}+\text { h.c. }\right)
$$




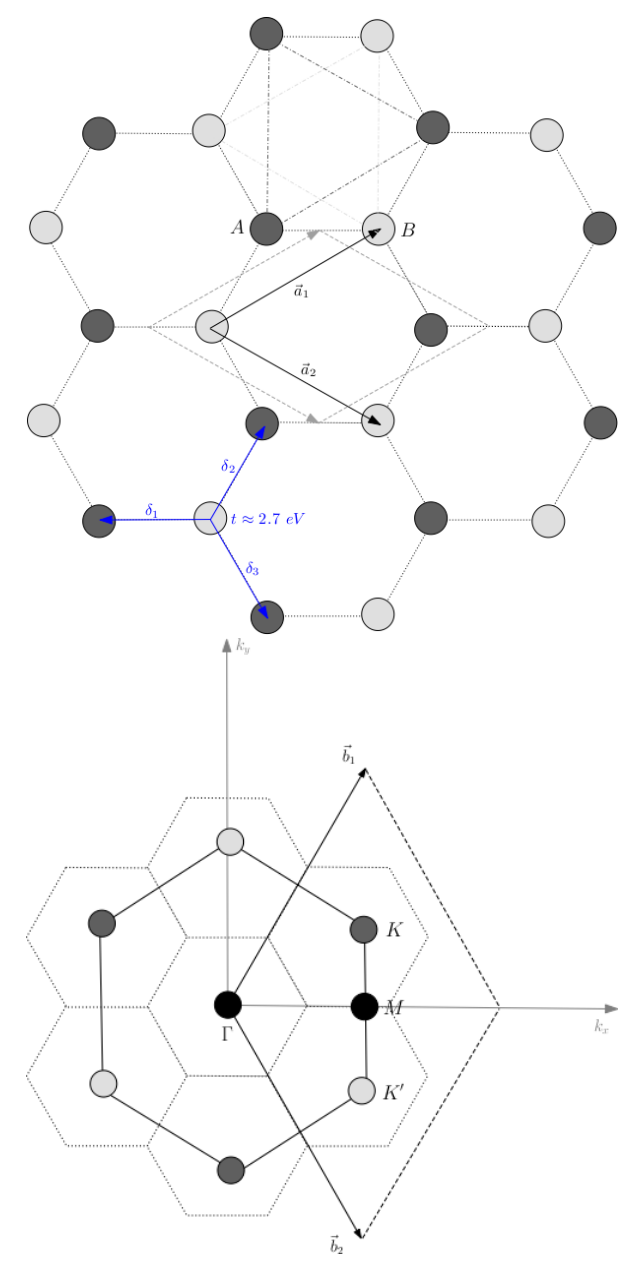

Figure 2.2: Hexagonal lattice representing the graphene structure (left), divided in two triangular lattices, $\mathrm{A}$ and $\mathrm{B}$, in which atoms $\mathrm{A}$ and $\mathrm{B}$ belong to each lattice, respectively. Vectors $\mathbf{a}_{1}$ and $\mathbf{a}_{2}$ are the lattice vectors and $\delta_{i}$ are the next-neighbor vectors (the transition amplitude between these are of the order $t \approx 2.7 \mathrm{eV}$ ). The unit-cell is described by the rhombic geometry. The reciprocal lattice (right) corresponds to the first BZ represented in $\mathbf{k}$ space. Vectors $\mathbf{b}_{1}$ and $\mathbf{b}_{2}$ are reciprocal vectors and $K$ and $K^{\prime}$ are special lattice points, which result in the atom differentiation of the two triangular lattices. Points $\Gamma$ and $M$ are high-symmetry k-points in the BZ. 
in which $t$ is the transition amplitude for the next-neighbor $(\approx 2.7 \mathrm{eV})$. The $a_{n}, b_{n+\delta}$ are Fermi operators and these represent electrons at the $A$ and $B$ sublattices, respectively. $\delta$ are vectors that are directed to the three next-neighbors of the site.

Considering the periodic boundary conditions of the system, which leads to the conversion of the real-space operators to plane-waves of reciprocal space (Fourier transform), one obtains

$$
a_{\mathbf{k}}=\frac{1}{\sqrt{N}} \sum_{n} e^{-\imath \mathbf{k} \cdot \mathbf{n}} a_{\mathbf{n}} ; \quad b_{\mathbf{k}^{\prime}}=\frac{1}{\sqrt{N}} \sum_{n} e^{-\imath \mathbf{k}^{\prime} \cdot(\mathbf{n}+\delta)} b_{\mathbf{n}+\delta}
$$

then

$$
H=\frac{t}{N} \sum_{\mathbf{n}, \delta, \mathbf{k}, \mathbf{k}^{\prime}} e^{-\imath \mathbf{k} \cdot \mathbf{n}} a_{\mathbf{k}}^{\dagger} e^{\imath \mathbf{k}^{\prime} \cdot(\mathbf{n}+\delta)} b_{\mathbf{k}^{\prime}}+\text { h.c. } .
$$

The sum of all the lattice sites, $n$, originates the Dirac delta function, such that, $\imath \delta\left(\mathbf{k}^{\prime}-\mathbf{k}\right)=1 / N \sum_{n} e^{\imath\left(\mathbf{k}^{\prime}-\mathbf{k}\right) n}$, therefore

$$
H=t \sum_{\mathbf{k}, \delta} e^{\imath \mathbf{k} \cdot \delta} a_{\mathbf{k}}^{\dagger} b_{\mathbf{k}}+h . c .=\sum_{\mathbf{k}} \epsilon_{\mathbf{k}}\left(e^{\imath \varphi_{\mathbf{k}}} a_{\mathbf{k}}^{\dagger} b_{\mathbf{k}}+e^{-\imath \varphi_{\mathbf{k}}} b_{\mathbf{k}}^{\dagger} a_{\mathbf{k}}\right),
$$

with $\epsilon_{\mathbf{k}}=t\left|f_{\mathbf{k}}\right|$ and $f_{\mathbf{k}}=\sum_{\delta} e^{\imath \mathbf{k} \cdot \delta}=\left|f_{\mathbf{k}}\right| e^{\imath \varphi_{\mathbf{k}}}$. The Hamiltonian can be written in a matrix form,

$$
H=\sum_{\mathbf{k}} \epsilon_{\mathbf{k}}\left[\begin{array}{ll}
a_{\mathbf{k}}^{\dagger} & b_{\mathbf{k}}^{\dagger}
\end{array}\right]\left[\begin{array}{cc}
0 & e^{\imath \varphi_{\mathbf{k}}} \\
e^{-\imath \varphi_{\mathbf{k}}} & 0
\end{array}\right]\left[\begin{array}{l}
a_{\mathbf{k}} \\
b_{\mathbf{k}}
\end{array}\right]=\sum_{\mathbf{k}} t \psi_{\mathbf{k}}^{\dagger}\left[\begin{array}{cc}
0 & f_{\mathbf{k}} \\
f_{\mathbf{k}}^{*} & 0
\end{array}\right] \psi_{\mathbf{k}} .
$$

Replacing its values by the coordinates in Eq. 2.110, one obtains

$$
f_{\mathbf{k}}=\sum_{\delta} e^{\imath \mathbf{k} \cdot \delta}=e^{\imath \mathbf{k} \cdot \delta_{1}}+e^{\imath \mathbf{k} \cdot \delta_{2}}+e^{\imath \mathbf{k} \cdot \delta_{3}}
$$

then

$$
f_{\mathbf{k}}=\exp \left[\imath\left(\frac{a k_{x}}{2}+\frac{\sqrt{3} a k_{y}}{2}\right)\right]+\exp \left[\imath\left(\frac{a k_{x}}{2}-\frac{\sqrt{3} a k_{y}}{2}\right)\right]+\exp \left[-\imath a k_{x}\right],
$$

thereby computing in Eq. 2.113, the eigenvalues: 


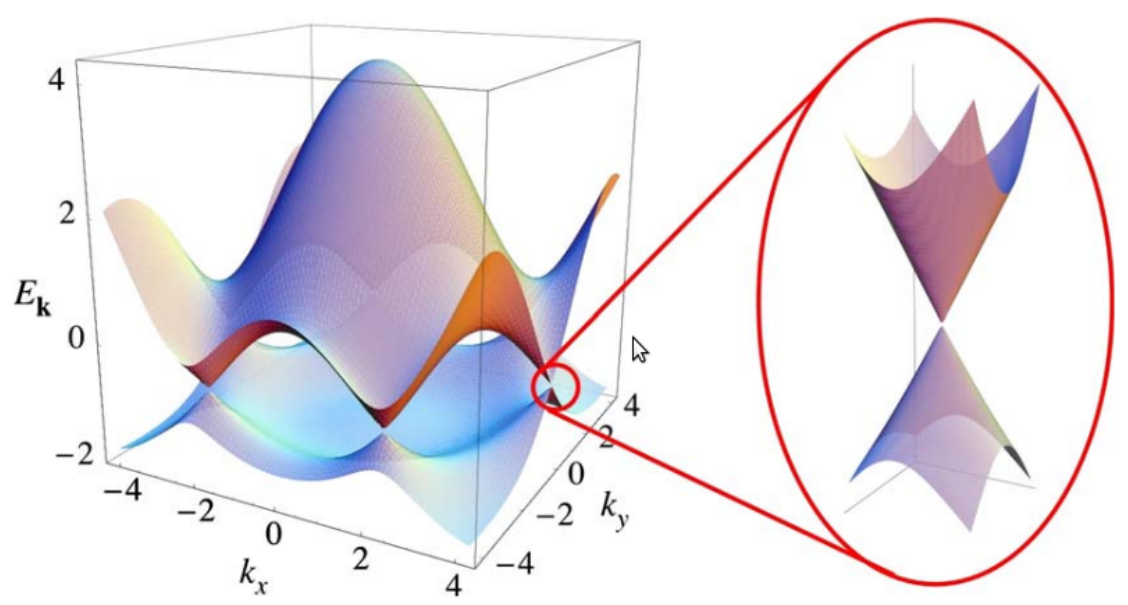

Figure 2.3: Energy dispersion, band structure highlighting the low energy limits. Note the linear band structure near the Dirac cones located at the points $K$ and $K^{\prime}$. Reproduced from Ref. [89].

$$
\epsilon_{k}= \pm t \sqrt{3+4 \cos \left[a k_{x} / 2\right] \cos \left[\sqrt{3} a k_{y} / 2\right]+2 \cos \left[a k_{x}\right]}
$$

we get the electronic dispersion relation, Fig. 2.3.

\section{Low Energy Limit}

Within the limit of low energies, $\epsilon_{k}=0$, close to the two nodal points, $K$ and $K^{\prime}$, one obtains a cone type energetic surface, leading to a relativistic-type electronic dynamics, which is described by the Dirac equation, Fig. 2.3. The position of these points in momentum space is given by

$$
K=\left(\frac{2 \pi}{3 a}, \frac{2 \pi}{3 \sqrt{3} a}\right), \quad K^{\prime}=\left(\frac{2 \pi}{3 a},-\frac{2 \pi}{3 \sqrt{3} a}\right) .
$$

The linear behavior of the dispersion relation can be better identified if we expand the Hamiltonian of Eq.2.113 around the points $K$ and $K^{\prime}$. Thus, 


$$
H=t\left[\begin{array}{cc}
0 & -\frac{3}{4}(-\imath+\sqrt{3}) a\left(q_{x}+\imath q_{y}\right) \\
-\frac{3}{4}(\imath+\sqrt{3}) a\left(q_{x}-\imath q_{y}\right) & 0
\end{array}\right] .
$$

The eigenvalues are linear in momentum

$$
\epsilon_{q}= \pm t a \frac{3}{2} \sqrt{q_{x}^{2}+q_{y}^{2}}= \pm \nu_{F}|\mathbf{q}|,
$$

on what $\nu_{F}=t a(3 / 2) \approx 1 \times 10^{6} \mathrm{~m} / \mathrm{s}$ is the Fermi velocity.

The above equation, Eq. 2.114, acquires a more perceptible form if if one applies a counter clockwise rotation of $\pi / 6$. Re-writing the moment variables as, $\kappa_{x}=\left(\sqrt{3} q_{x}\right) / 2-q_{y} / 2$ and $\kappa_{y}=\left(q_{x} / 2\right)+\left(\sqrt{3} q_{y}\right) / 2$, one obtains

$$
H_{K}=-\frac{3}{2} a t\left(\begin{array}{cc}
0 & \kappa_{x}+\imath \kappa_{y} \\
\kappa_{x}-\imath \kappa_{y} & 0
\end{array}\right)=-\frac{3}{2} a t\left[\sigma_{x} \kappa_{x}-\sigma_{y} \kappa_{y}\right],
$$

where $\sigma_{x}$ and $\sigma_{y}$ are the Pauli matrices, representing $\left.\sigma=\left(\sigma_{x}, \sigma_{y}\right)\right)$. Please note that the respective conjugate is $\sigma^{*}=\left(\sigma_{x}^{*}, \sigma_{y}^{*}\right)=\left(\sigma_{x},-\sigma_{y}\right)$; thus one gets

$$
H_{K}=-\nu_{F} \sigma^{*} \cdot \kappa .
$$

At the point $K^{\prime}$, the calculations are obtained analogously by applying a counter-clockwise rotation of $-\pi / 6$, yielding

$$
H_{K^{\prime}}=-\frac{3}{2} a t\left(\begin{array}{cc}
0 & \kappa_{x}-\imath \kappa_{y} \\
\kappa_{x}+\imath \kappa_{y} & 0
\end{array}\right)=-\frac{3}{2} a t\left[\sigma_{x} \kappa_{x}+\sigma_{y} \kappa_{y}\right] .
$$

In that case, one obtains

$$
H_{K^{\prime}}=-\nu_{F} \sigma \cdot \kappa .
$$

Both Hamiltonians, Eqs. 2.116 and Eq. 2.115, are decoupled, and have been obtained with the relativistic Dirac Hamiltonian [13]. The corresponding representation in a matrix form is then

$$
H=-\nu_{F} \sum_{\kappa}\left(\psi_{K, \kappa}^{\dagger} \sigma^{*} \cdot \kappa \psi_{K, \kappa}+\psi_{K^{\prime}, \kappa}^{\dagger} \sigma \cdot \kappa \psi_{K^{\prime}, \kappa}\right),
$$

wherein wavefunctions are the operators defined in Eq. 2.111, so that 


$$
\Psi_{\kappa}=\left(\begin{array}{c}
\psi_{K, \kappa} \\
\psi_{K^{\prime}, \kappa}
\end{array}\right)=\left(\begin{array}{c}
a_{K, \kappa} \\
b_{K, \kappa} \\
a_{K^{\prime}, \kappa} \\
b_{K^{\prime}, \kappa}
\end{array}\right)
$$

In this way, the Hamiltonian of Eq. 2.117 then becomes

$$
H=-\nu_{F} \sum_{\kappa} \Psi_{\kappa}^{\dagger}\left(\begin{array}{cccc}
0 & \kappa_{x}-\imath \kappa_{y} & 0 & 0 \\
\kappa_{x}+\imath \kappa_{y} & 0 & 0 & 0 \\
0 & 0 & 0 & \kappa_{x}+\imath \kappa_{y} \\
0 & 0 & \kappa_{x}-\imath \kappa_{y} & 0
\end{array}\right) \Psi_{\kappa}
$$

If we introduce the matrices $\alpha_{i}$ in the form [90]

$$
\begin{aligned}
\alpha_{1}=\left(\begin{array}{cccc}
0 & 1 & 0 & 0 \\
1 & 0 & 0 & 0 \\
0 & 0 & 0 & 1 \\
0 & 0 & 1 & 0
\end{array}\right) ; & \alpha_{2}=\left(\begin{array}{cccc}
0 & -\imath & 0 & 0 \\
\imath & 0 & 0 & 0 \\
0 & 0 & 0 & \imath \\
0 & 0 & -\imath & 0
\end{array}\right) ; \\
\alpha_{3}=\left(\begin{array}{cccc}
1 & 0 & 0 & 0 \\
0 & -1 & 0 & 0 \\
0 & 0 & 1 & 0 \\
0 & 0 & 0 & -1
\end{array}\right) ; & \alpha_{4}=\left(\begin{array}{cccc}
0 & 0 & 0 & -1 \\
0 & 0 & 1 & 0 \\
0 & 1 & 0 & 0 \\
-1 & 0 & 0 & 0
\end{array}\right)
\end{aligned}
$$

then the Hamiltonian can be further expressed as

$$
H=-\nu_{F} \sum_{\kappa} \Psi_{\kappa}^{\dagger}\left(\alpha_{1} \kappa_{x}+\alpha_{2} \kappa_{y}\right) \Psi_{\kappa}
$$

For graphene, the $2 D+1$ Hamiltonian, is obtained noting that $\gamma_{1}=\gamma_{0} \alpha_{1}$, $\gamma_{2}=\gamma_{0} \alpha_{2}$ and $\gamma_{0}=\alpha_{4}$. In such a case, we can write

$$
H=\imath \nu_{F} \sum_{\kappa} \bar{\Psi}_{\kappa} \gamma^{\mu} \partial_{\mu} \Psi_{\kappa}
$$

Graphene comprises a relativistic dynamics described by the Dirac algebra, as reflected in Dirac matrices. It is noted however, that in $\mathrm{QED}_{2+1}, \gamma_{3}$ is 
not used in the construction of Hamiltonian, since the electronic dynamics in this material is restricted to 2D. Eq. 2.119 shows that graphene involves a relativistic electronic dynamic type described by a Dirac equation.

Graphene is a material with an electronic dynamics of Dirac relativistic type, which is striking in condensed matter physics. The remarkable distinction of graphene is that the relativistic effects are genuine systems of quantum field theory. Moreover, one may have the possibility of studying the effects of localization of the wavefunction caused by impurity disorder.

\subsubsection{DOS in Graphene}

The DOS of the Graphene system can then be obtained using the equation:

$$
\rho_{0}=-\frac{1}{\pi} \operatorname{Im} \operatorname{Tr} \hat{G}^{(0)} .
$$

By definition, we write

$$
\hat{G}^{(0)}(\varepsilon, k)=\left(\varepsilon-\hat{H}_{0}\right)^{-1}=\frac{1}{N} \sum_{k} \frac{\varepsilon \hat{1}+\nu_{F}\left(\gamma^{1} k_{x}+\gamma^{2} k_{y}\right)}{\varepsilon^{2}-\varepsilon_{\mathbf{k}}^{2}} .
$$

Then, the diagonal element of the GF $g^{(0)}$, in the vicinity of the Dirac point is given in Ref. [91]. Indeed, by integrating around the Dirac cones $\sum_{k} \rightarrow$ $\int_{\text {Dirac Cone }} d \mathbf{k}$, for a linear scattering behavior, we obtain

$$
g^{(0)}=\frac{a^{2}}{2 \pi} \int_{0}^{2 \pi} \partial \theta \int_{0}^{2 \sqrt{\pi} / a} \partial k \frac{k \varepsilon}{\varepsilon^{2}-(t a k)^{2}}=\varepsilon \ln \frac{\varepsilon^{2}}{1-\varepsilon^{2}}-\imath \pi|\varepsilon|,
$$

where we choose the energy unit in such a way that the bandwidth $2 \sqrt{\pi} t=1$ [91]. Therefore the DOS is given by

$$
\rho_{0}=-\frac{1}{\pi} \operatorname{Im} \operatorname{Tr} \hat{G}^{(0)}=-\frac{1}{\pi} \operatorname{Im} g^{(0)}=|\varepsilon|
$$

whose representation is in the graph of Fig. 2.4 


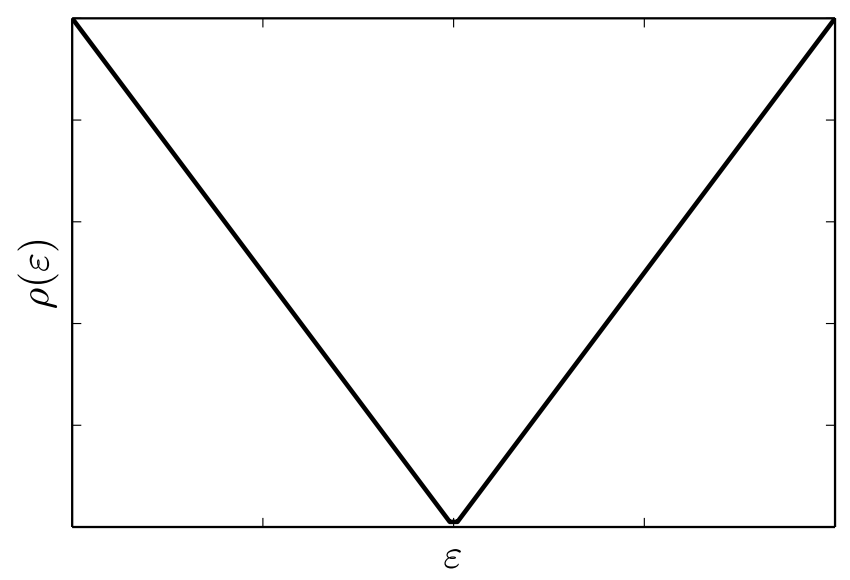

Figure 2.4: The density of states, for pure graphene

\section{Impurities in graphene - Disordered Systems}

For a disordered system, the most reliable characteristics for relevant observations, are described by the 'self-mediated' GFs whose values do not depend on the particular realization of disorder. In other words, this means that, to all particular realization for disorder, the GFs become equal to the mean values over the disorder [92].

For the present study, related to low concentration of impurities, we will apply the Lifshitz disordered model, which refers to the randomness on the spatial distribution of impurities - better suited to low concentration of defects.

Impurities will be considered within the Tight-Binding description by adding a local energy term of the form:

$$
H_{i m p}=\sum_{\langle\alpha, p\rangle} V_{p} c_{\alpha, p}^{\dagger} c_{\alpha, p}
$$

where, $V_{p}$, is the random potential, $\alpha$, represents two sub-lattices and $p$ is the site of the respective sub-lattice. Rewriting the operators, to explicitly represent electrons at the $A$ and $B$ sub-lattices, we have: 


$$
H_{i m p}=\sum_{\langle p\rangle} V_{p}\left(a_{p}^{\dagger} a_{p}+b_{p}^{\dagger} b_{p}\right)
$$

According to the notation of Ref. [91] we can write the Hamiltonian as a spinor. Therefore we have

$$
H_{i m p}=\sum_{k, k^{\prime},\langle p\rangle} \frac{V_{p}}{N} e^{\imath\left(k-k^{\prime}\right) p} \Psi_{k}^{\dagger} \tau_{0} \Psi_{k^{\prime}}
$$

where $\Psi=\left(\begin{array}{l}a_{k} \\ b_{k}\end{array}\right)$.

One of the theoretical objects mostly employed to study impurities is the Tmatriz, method $\hat{T}(\varepsilon)$. In this work, all the effects induced by the impurity will be considered within the T-matrix method, of which standard perturbation theory results in an infinite sum of perturbed Gfs

$$
\begin{aligned}
\hat{T}(\varepsilon) & =\hat{V}+\hat{V} \hat{G}^{0} \hat{V}+\hat{V} \hat{G}^{0} \hat{V} \hat{G} \hat{V}+\cdots \\
& =\frac{\hat{V}}{1-\hat{V} \hat{G}}
\end{aligned}
$$

In case of assuming a small concentration of impurities, $c_{i}=N_{i} / N$, the GF can be written as

$$
\hat{G}(\varepsilon, k)=\hat{G}_{k}^{0}+\hat{G}_{k}^{0} \hat{T}(\varepsilon) \hat{G}_{k}
$$

being this equation only valid up to first order of $c_{i}$, meaning that this form only considers the multiple scattering of electrons by the effect of only one impurity. From Eq. 2.122, and replacing the respective result, T-matrix is written:

$$
T(\varepsilon)=\frac{V}{1-V\left(\operatorname{Re} g^{(0)}-\imath \pi|\varepsilon|\right)},
$$

therefore

$$
\operatorname{Im} T(\varepsilon)=\frac{V^{2} \pi|\varepsilon|}{\left(1-V \operatorname{Re} g^{(0)}\right)^{2}+(V \pi \varepsilon)^{2}}
$$




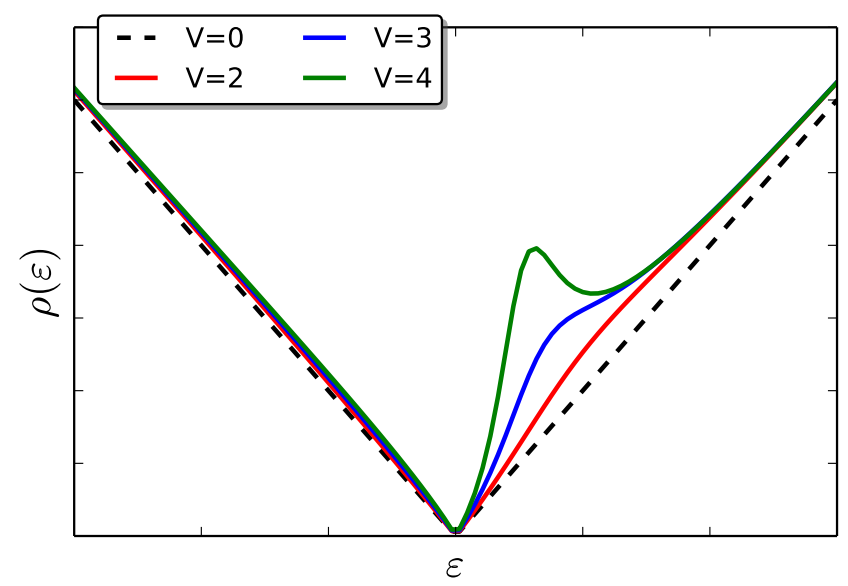

Figure 2.5: DOS and T-matrix in graphene as a function of energy, where different perturbation potentials are applied with $c_{i}=0.01$ of impurity concentrations.

for sufficiently large $V$, a peak is observed in the DOS (see Fig. 2.5).

\subsubsection{Vacancy defects}

Vacancies are an example of possible defects that can be introduced in the lattice by proton irradiation. A vacancy can be considered as extreme cases of the local potential when $V \rightarrow \infty$, being the potential considered in the unitary limit. Therefore, the T-matrix will transform as

$$
\lim _{V \rightarrow \infty} T(\varepsilon)=-\frac{1}{g^{(0)}(\varepsilon)},
$$

when $g^{(0)}(\varepsilon=0)=0$, the DOS will tend to $\infty$, as observed in Fig. 2.6.

To calculate the DOS when dealing with vacancies, in the extreme case when $V \rightarrow \infty$, the T-matrix calculation, as previously obtained, is not rigorous because the resonance peak will diverge to infinity.

In what concerns the vacancy problem, one may still observe the divergence of the DOS at $\varepsilon=0$ (see Fig. 2.6), even by calculating through the self-consistent method [22]. Nevertheless, this method is more reliable, allowing for one to 


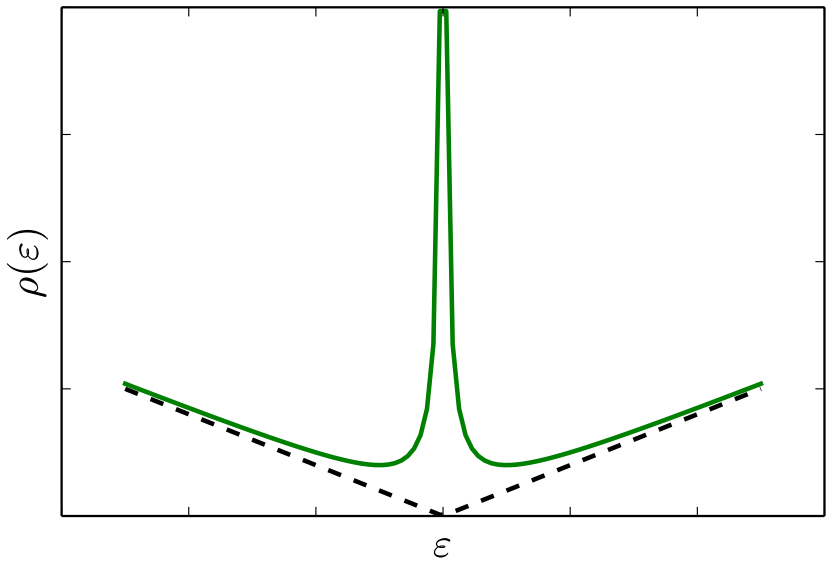

Figure 2.6: Effect of the vacancy impurity on the DOS (green solid line) where a divergence at $\epsilon=0$ is observed. The dashed black line refers to the DOS of the pure graphene system.

reproduce in better agreement experimental results and compare with other data obtained through theoretical and computational analysis [93]. 


\subsubsection{Strain-Induced Effects of Deformed Graphene}

In this section we will present an analytical solution of the relativistic Dirac equation defined in the framework of quantum field theory in curved space and will apply it to study the electronic properties of deformed monolayer graphene. We will obtain as solution the Dirac oscillator equation, where an effective vector potential term naturally appears. Such a term describes a pseudo-magnetic field that emerges due to the initially defined curvature, and influences the dynamics of the charge carriers, as if these were under the influence of an applied external magnetic field.

The flexibility of manipulating monolayer graphene allows one to engineer the electronic and optical properties by inducing local lattice deformations to control the strain distribution of the atom-thick material (straintronics and curvatronics). Inspite the electron dynamics in graphene is described by a twodimensional (2D) Dirac equation, one should expect deviations from a $2 \mathrm{D}$ flat surface. In fact, structural corrugations and ripples have been experimentally observed in suspended graphene and ab initio simulations have shown that ripples tend to emerge spontaneously due to thermal fluctuations [89, 94].

When the monolayer sheet is perturbed under strained geometric curvatures, the behaviour of the charge carriers will mirror those occurring under the influence of an applied out-of-plane magnetic field [95, 96, 97]. It has been observed that inducing large enough stretching/strain promotes tiny triangular bubbles in the material sheet that causes pseudomagnetic fields localized inside the bubbles. These fields can reach as high as $300 \mathrm{~T}$, i.e. well beyond field values currently obtained with stable laboratory magnets[95, 97]. Such strong fields result in the localization of the electronic states, leading to observable phenomena, such as pseudo-quantum Hall effects and Landau levels.

Since the linear band dispersion of graphene presents degenerate valleys, where the valence and conduction bands touch at the Dirac points, the effect of lattice deformations on electrons is equivalent to that of an effective gauge field. As a consequence, homogeneous deformations causes small shifts in momentum of the Dirac cones, whereas inhomogeneous strain influences the electron motion, similarly to a valley-dependent effective pseudo-magnetic field[97]. Such effects could enable potential applications, mainly in the fields of valleytronics, in which electron separation between different valleys in the electronic band dispersion can occur [97].

Gauge fields, arising due to elastic deformations in the presence of inhomogeneous external stress, have already been realized more than 20 years ago by 
Kane and Mele[98]. However, the correlation between a particular topological strain and the corresponding pseudo-magnetic field is not straightforward, and thus several theoretical studies have attempted to use strain-engineering techniques to generate homogeneous magnetic fields[94, 99, 100, 101, 102].

Moreover, since induced strain modifies the Fermi level, correlation between strain and deformation engineering, with the possibility of tuning the energy band gaps in the graphene electronic spectrum, has also been evidenced $[95,101]$. This issue is still one of the most debated challenges in graphene electronics. Only quite recently it has been found through numerical calculations that the combination of shear strain and uniaxial tensile deformations seem to be the easiest form of band gap engineering [103], enabling band gap widths of up to $6 \mathrm{eV}$. Curved 2D systems also present another form of inducing compressive and tensile strain in the lattice. Therefore the magnitudes of the strain percentages depend on the topology and on the curvature radius of the induced curved surface [104].

It is important to note that applying isotropic or uniaxial strains it is not possible to break inversion symmetry of the graphene lattice, therefore not being possible to induce a band gap opening. Alternative local strains may allow the possibility for band gap engineering. Local strains can naturally occur from growing conditions on substrates with different structural local deformations (grooves, wrinkles, steps, etc.), thereby generating different strain profiles [103].

Two main theoretical approaches based on effective gauge fields are usually employed to study the electronic properties of strain/deformation induced modifications in graphene. The most standard method is based on tight-binding models and focuses on the low-energy continuum limit accounting for displacements of carbon atoms in a strained sheet. The second approach is based on quantum field theories of curved space, which starts off with a low-energy effective Dirac equation for graphene and by considering a curved space metric, one obtains geometry-induced gauge fields.

Initially the Dirac oscillator had been introduced in the context of many body theory, mainly in connection with quark confinement models in quantum chromodynamics [105]. Within the non-relativistic limit, the Dirac oscillator is reduced to a simple harmonic oscillator with a strong spin-orbit coupling term. Currently, a wide variety of works have directed the physics of the Dirac oscillator when monolayer graphene is subject to an external applied magnetic field[106, 107]. However, in the present work, we will verify that a pseudomagnetic field naturally emerges when graphene presents a deformation of the 
type of $z(x, y)$, which in turn produces the same space group symmetry as the pristine flat monolayer sheet of graphene, thus showing it to be a naturally occurring deformation of the system.

The main motivation to search for deformations on the monolayer sheet is because graphene-type materials inevitably are subject to out-of-plane deformations, and described by the function that we will demonstrate in this work. The physics described by the Dirac oscillator equation allows to interpret many experimental results carried out in graphene-type materials. As a consequence of the Dirac harmonic oscillator equation, it is possible to address the physics of the Landau levels and also consider the theoretical framework of JaynesCummings for the relevant optical properties of curved graphene [108, 109].

Within the framework of the relativistic electron in curved space, the action can be written ${ }^{1}$ as

$$
S=\int d^{3} x \sqrt{g} \bar{\Psi}(x)\left\{\Gamma^{\mu} D_{\mu}-M\right\} \Psi(x)
$$

where $\Psi(x)$ is the Dirac field, $g=\operatorname{det}\left(g_{\mu \nu}\right)$ with $g_{\mu \nu}$ being the metric tensor. The Dirac equation in a curved space-time is written as $\left\{\imath \Gamma^{\mu} D_{\mu}-M\right\} \Psi(x)=0$, where the Dirac matrices in curved space-time are $\Gamma^{\mu}=e_{A}^{\mu} \gamma^{A}$. The local Lorentzian frame is defined by $e_{A}^{\mu}$ with space-time interval given by $d s^{2}=$ $\eta_{A B} \theta^{A} \theta^{B}$, being $\eta_{A B}$ the Minkowski metric and $\theta^{A}=e_{A}^{\mu} d x^{\mu}$.

The covariant derivative is:

$$
D_{\mu}=\partial_{\mu}+\frac{1}{4} \omega_{\mu}^{A B} \omega_{A B}
$$

with $\omega_{A B}=\frac{1}{2}\left(\gamma_{A} \gamma_{B}-\gamma_{B} \gamma_{A}\right)$ and the spin connection is

$$
\begin{aligned}
\omega_{\mu}^{A B} & =\frac{1}{2} e^{\nu A}\left(\partial_{\mu} e_{\nu}^{B}\right. \\
& \left.-\partial_{\nu} e_{\mu}^{B}\right)-\frac{1}{2} e^{\nu B}\left(\partial_{\mu} e_{\nu}^{A}-\partial_{\nu} e_{\mu}^{A}\right) \\
& -\frac{1}{2} e^{\rho A} e^{\sigma B}\left(\partial_{\rho} e_{\sigma C}-\partial_{\sigma e_{\rho C}} e_{\mu}^{C}\right.
\end{aligned}
$$

${ }^{1}$ 'Es en la acción y solo en la acción cuando la virtud se destaca' Cicerön 

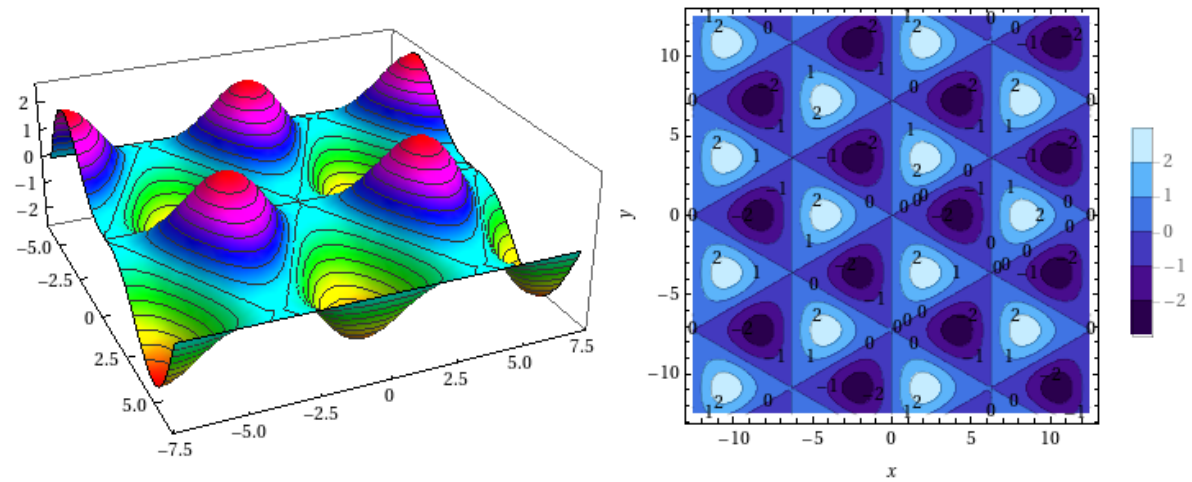

Figure 2.7: 3D representation (left) and contour plot (right) of the geometric deformation pattern of graphene defined by Eq. 2.124 .

In the particular case of a time independent metric, one can choose $\theta^{0}=d t$ and $\Gamma^{0}=\gamma^{0}$, where $\gamma^{0}$ is the flatland Dirac matrix [94].

We consider the out-of-plane deformation of the form (Fig. 2.7):

$$
\begin{aligned}
z(x, y)= & \frac{1}{2} A \cos [k(x+y)] \\
& \cos [\sqrt{3} k(-x+y)-k(x+y)] \\
& \cos [\sqrt{3} k(-x+y)+k(x+y)] \\
& \sin [k(x+y]
\end{aligned}
$$

where $A$ is the deformation amplitude and $k$ refers to the modulation frequency of the deformation. For a small deformation parameter $\eta=A k<<1$, we obtain

$$
\Gamma^{1}(x, y)=\gamma^{1}, \quad \Gamma^{2}(x, y)=\gamma^{2},
$$

with the spin connection expression written as

$$
\omega_{1}^{12}(x, y)=-A^{2} k^{4} x, \quad \omega_{2}^{12}(x, y)=-A^{2} k^{4} x .
$$

After rearranging the terms, we obtain the Dirac oscillator expression 


$$
\imath\left\{\gamma^{0} \partial_{0}+\gamma^{x}\left(\partial_{x}+y B\right)+\gamma^{y}\left(\partial_{y}-x B\right)\right\} \Psi=0
$$

where $B$ is a constant that represents the pseudo-magnetic field which emerges due to the defined out-of-plane deformation. For this out-of-plane deformation it is possible to obtain the Dirac harmonic oscillator equation, where Landau levels can here be addressed. Such an expression had initially been introduced in the context of quark confinement models in the fields of quantum chromodynamics [105].

Arias and co-workers [110] have studied both in-plane and out-of-plane deformations and have obtained a closed expression for the effective gauge field due to arbitrary nonuniform sheet deformations. Moreover, these authors reveal a relation between the local pseudo-magnetic field and the intrinsic scalar curvature; i.e. the Riemann curvature [110].

The Dirac oscillator expression decouples in a set of equations of the form:

$$
\begin{aligned}
& E\left|\Psi_{a}\right\rangle=\left[p_{x}+\imath B_{x}-\imath p_{y}+B_{y}\right]\left|\psi_{b}\right\rangle \\
& E\left|\Psi_{b}\right\rangle=\left[p_{x}-\imath B_{x}+\imath p_{y}+B_{y}\right]\left|\psi_{a}\right\rangle
\end{aligned}
$$

Re-writing the equation in complex notation we thus obtain:

$$
H_{D}(\lambda)=\left(\begin{array}{cc}
0 & 2 p_{z}+\imath B_{z} \\
2 \bar{p}_{z}-\imath B_{z} & 0
\end{array}\right)
$$

in which the Dirac oscillator equation can be written in the usual creation and annihilation operators, reformulated in the complex formalism (JaynesCummings model) [106], as:

$$
H_{D} \propto\left(\begin{array}{cc}
0 & \bar{a}_{z} \\
a_{z} & 0
\end{array}\right)
$$

Previous work [94] has considered that deformations result from the Mathieu equations that describe the Kaptiza pendulum, therefore the motivation here is to try and search for a more generalized deformation, in such a way as to obtain the Dirac oscillator. 
Chapter 3

\section{Effects of extended impurity perturbation in d-wave superconductor}




\title{
Effects of extended impurity perturbation in a $d$-wave superconductor
}

\author{
Yu. G. Pogorelov and M. C. Santos \\ CFP/Departamento de Física, Universidade do Porto, 4169-007 Porto, Portugal \\ (Received 21 February 2004; revised manuscript received 2 August 2004; published 21 January 2005)
}

\begin{abstract}
We describe the effects of electronic perturbation distributed on nearest-neighbor sites to the impurity center in a planar $d$-wave superconductor, in approximation of circular Fermi surface. The behavior previously reported for pointlike perturbation and square Fermi surface, the quasiparticle density of states $\rho(\varepsilon)$ can display a resonance inside the gap and asymptotically vanishes at $\begin{gathered}\varepsilon \\ 0\end{gathered}$ as $\rho \quad \varepsilon / \ln ^{2} \varepsilon$. Unique features are weak antiresonances from low-symmetry representations of nonlocal perturbation. The local suppression of superconducting (SC) order parameters in this model is found to be somewhat weaker than for an equivalent pointlike (nonmagnetic) perturbation and much weaker than for a spin-dependent (extended) perturbation. The developed approach can be used for a wide class of nonlocal impurity perturbations in superconductors.
\end{abstract}

\section{INTRODUCTION}

The study of the density of states (DOS) in high- $T_{c}$ superconducting (SC) metal oxides has motivated many theorists and experimentalists through the last years, because it defines such fundamental physical parameters as the quasiparticle conductivity $\sigma$, the penetration length $\lambda$, the electronic specific heat, etc. This study is guided by the facts that (i) the charge carriers are practically confined to the $\mathrm{CuO}_{2}$ planes ${ }^{1}$ and characterized by two-dimensional (2D) wave vectors $\mathbf{k}$ $=\left(k_{x}, k_{y}\right)$ and (ii) the SC order parameter has $d$-wave symmetry $^{2}$ with four nodal points $\mathbf{k}_{i}=\left( \pm k_{F} / \sqrt{2}, \pm k_{F} / \sqrt{2}\right)$, where the Fermi surface crosses the nodal directions $k_{x}$ $= \pm k_{y}$; along these directions the SC gap function $\Delta_{\mathrm{k}}$ turns zero, and the quasiparticle dispersion law $E_{\mathbf{k}}=\sqrt{\xi_{\mathbf{k}}^{2}+\Delta_{\mathbf{k}}^{2}}$ coincides with that of normal metal, $\xi_{\mathbf{k}}$. Another important factor is that the high- $T_{c}$ materials are the so-called "doped metals" (also called doped insulators or doped semiconductors), where the Fermi energy $\varepsilon_{F}$ is defined by the density of charge carriers, introduced by the doping process. ${ }^{3}$ This very process creates the scattering centers for quasiparticles, due to random Coulomb fields from ionized dopants. Other scatterers, not related to the density of carriers, can be additionally introduced, and all of them can produce considerable effects on the system physical properties. ${ }^{4}$

In particular, some resonances can emerge in the quasiparticle spectrum with $d$-wave gap symmetry, ${ }^{5,6}$ even at low concentrations of impurities. Such resonance manifests itself in a maximum of DOS at a certain energy $\varepsilon_{\text {res }}<\Delta$ $=\max _{\mathbf{k}}\left|\Delta_{\mathbf{k}}\right|$, as well as in logarithmic suppression of DOS at $\varepsilon \quad \varepsilon_{\text {res }}$ (Ref. 7) (the energies being referred to $\varepsilon_{F}$ ). These conclusions can be directly compared to experimental results, as those obtained in the scanning tunneling microscopy experiments. ${ }^{10}$

The perturbation that impurities introduce into the electronic subsystem of crystal, depend either on their positions with respect to the lattice and on the potential they produce on nearest matrix sites. Within the simplest possible model, where an impurity only disturbs a single site in the lattice, ${ }^{5,6,11-14}$ the potential is characterized by a single perturbation parameter. This pointlike perturbation model al- lows one to obtain a simple solution for the quasiparticle DOS in terms of their Green's functions, leading to the above-mentioned possibility of low-energy resonances. However, in reality, the impurity perturbations in high- $T$ materials are not exactly pointlike but rather extended to lattice sites neighboring the impurity center. The opposite limit to the pointlike perturbation is when the perturbed area is much bigger of the Fermi wavelength and can be treated quasiclassically, ${ }^{15}$ but it hardly applies to real atomic substitutes in high- $T_{c}$ systems where the perturbation extends to few nearest neighbors of the impurity site. One important question in this respect is how robust are the results of pointlike approximation to the spatial extent and geometry of impurity perturbation..$^{5}$ In particular, the linkage of $d$-wave SC order to nearest-neighbor pairing rather than to on-site pairing can generate new features, e.g., the extended impurity gets to perturb diagonal and off-diagonal observable values in different ways (see below). Recently, an example of such an extended impurity center was considered, ${ }^{16}$ but for a specific spin-dependent perturbation which does not have a pointlike counterpart. Here we use a similar approach to compare the effects of pointlike and extended perturbations of otherwise identical (spin-independent) structure, and also to compare them with the spin-dependent perturbation. These studies could prepare a more flexible base for treating the dynamics of disordered high- $T_{c}$ materials.

Usually, the low-energy excitations in $d$-wave systems with impurities are considered starting from a tight-binding spectrum in the simplest nearest-neighbor approximation, $\xi_{\mathrm{k}}=2 t\left(2-\cos a k_{x}-\cos a k_{y}\right)$, and using a certain parametrization in the vicinity of nodal points. Thus, in the popular approach proposed by Lee, ${ }^{11}$ one expands the difference $\mathbf{k}$ $-\mathbf{k}_{i}$ in the local axes, $\mathbf{e}_{i, 1}=\mathbf{k}_{i} / k_{\mathrm{F}}$ and $\mathbf{e}_{i, 2}=\left(\mathbf{e}_{i+1,1}-\mathbf{e}_{i-1,1}\right) / 2$ [Fig. 1(a)], and then approximates the spectrum components as,

$$
\xi_{\mathbf{k}}=\hbar_{F} k_{1}, \Delta_{\mathbf{k}}=\hbar_{2} k_{2},
$$

with two characteristic velocities $2 \quad F$. However, this "square" geometry is a good approximation only for low quasiparticle energies $\left(E_{\mathbf{k}} \quad \Delta=\max _{\mathbf{k}}\left|\Delta_{\mathbf{k}}\right|\right)$ [except for the 


\section{Effects of extended impurity perturbation in d-wave superconductor}

Yu.G. Pogorelov and M.C. Santos

CFP/Departamento de Física, Universidade do Porto, 4169-007 Porto, Portugal

We describe the effects of electronic perturbation distributed on nearest-neighbor sites to the impurity center in a planar $d$-wave superconductor, in approximation of circular Fermi surface. The behavior previously reported for pointlike perturbation and square Fermi surface, the quasiparticle density of states $\rho(\varepsilon)$ can display a resonance inside the gap and asymptotically vanishes at $\varepsilon \rightarrow 0$ as $\rho \sim \varepsilon / \ln ^{2} \varepsilon$. Unique features are weak anti resonances from lowsymmetry representations of nonlocal perturbation. The local suppression of superconducting (SC) order parameters in this model is found to be somewhat weaker than for an equivalent pointlike (nonmagnetic) perturbation and much weaker than for a spin-dependent (extended) perturbation. The developed approach can be used for a wide class of nonlocal impurity perturbations in superconductors.

\section{Introduction}

The study of the density of states (DOS) in high- $\mathrm{T}_{c}$ superconducting (SC) metal oxides has motivated many theorists and experimentalists through the last years, because it defines such fundamental physical parameters as the quasiparticle conductivity $\sigma$, the penetration length $\lambda$, the electronic specific heat, etc. This study is guided by the facts that (i) the charge carriers are practically confined to the $\mathrm{CuO}_{2}$ planes [111] and characterized by two-dimensional (2D) wave vectors $\mathbf{k}=\left(k_{x}, k_{y}\right)$ and (ii) the SC order parameter has $d$-wave symmetry [112] with four nodal points $\mathbf{k}_{i}=\left( \pm k_{\mathrm{F}} / \sqrt{2}, \pm k_{\mathrm{F}} / \sqrt{2}\right)$, where the Fermi surface crosses the nodal directions $k_{x}= \pm k_{y}$; along these directions the SC gap function $\Delta_{\mathbf{k}}$ turns zero, and the quasiparticle dispersion law $E_{\mathbf{k}}=\sqrt{\xi_{\mathbf{k}}^{2}+\Delta_{\mathbf{k}}^{2}}$ coincides with that of normal metal, $\xi_{\mathbf{k}}$. Another important factor is that the high- $\mathrm{T}_{c}$ materials are the so-called "doped metals" (also called doped insulators or doped semiconductors), where the Fermi energy is defined by the density of charge carriers, introduced by the doping process.[113] This very process creates the scattering centers for quasiparticles, due to random Coulomb fields from ionized dopants. Other scatterers, not related to the density of carriers, can be additionally introduced, and all of them can produce considerable effects on the system physical properties.[114] 
In particular, some resonances can emerge in the quasiparticle spectrum with $d$-wave gap symmetry, $[87,115]$ even at low concentrations of impurities. Such resonance manifests itself in a maximum of DOS at a certain energy $\varepsilon_{\text {res }}<$ $\Delta=\max _{\mathbf{k}}\left|\Delta_{\mathbf{k}}\right|$, as well as in logarithmic suppression of DOS at $\varepsilon \ll \varepsilon_{\text {res }}$ (Ref. [116]) (the energies being referred to $\varepsilon_{\mathrm{F}}$ ). These conclusions can be directly compared to experimental results, as those obtained in the scanning tunneling microscopy experiments.[117]

The perturbation that impurities introduce into the electronic subsystem of crystal, depend either on their positions with respect to the lattice and on the potential they produce on nearest matrix sites. Within the simplest possible model, where an impurity only disturbs a single site in the lattice, [87, $115,118,119,120,121]$ the potential is characterized by a single perturbation parameter. This pointlike perturbation model allows one to obtain a simple solution for the quasiparticle DOS in terms of their Green's functions, leading to the above-mentioned possibility of low-energy resonances. However, in reality, the impurity perturbations in high- $\mathrm{T}_{c}$ materials are not exactly pointlike but rather extended to lattice sites neighboring the impurity center. The opposite limit to the pointlike perturbation is when the perturbed area is much bigger of the Fermi wavelength and can be treated quasiclassically,[122] but it hardly applies to real atomic substitutes in high- $\mathrm{T}_{c}$ systems where the perturbation extends to few nearest neighbors of the impurity site. One important question in this respect is how robust are the results of pointlike approximation to the spatial extent and geometry of impurity perturbation.[123] In particular, the linkage of d-wave SC order to nearest-neighbor pairing rather than to on-site pairing can generate new features, e.g., the extended impurity gets to perturb diagonal and off-diagonal observable values in different ways (see below). Recently, an example of such an extended impurity center was considered,[124] but for a specific spin-dependent perturbation which does not have a pointlike counterpart. Here we use a similar approach to compare the effects of pointlike and extended perturbation sof otherwise identical (spinindependent) structure, and also to compare them with the spin-dependent perturbation. These studies could prepare a more flexible base for treating the dynamics of disordered high- $T_{c}$ materials.

Usually, the low-energy excitations in $d$-wave system swith impurities are considered starting from a tight-binding spectrum in the simplest nearest-neighbor approximation, $\xi_{\mathbf{k}}=2 t\left(2-\cos a k_{x}-\cos a k_{y}\right)$, and using a certain parametrization in the vicinity of nodal points. Thus, in the popular approach proposed by Lee,[118] one expands the difference $\mathbf{k}-\mathbf{k}_{i}$ in the local axes, $\mathbf{e}_{i, 1}=\mathbf{k}_{i} / k_{\mathrm{F}}$ 

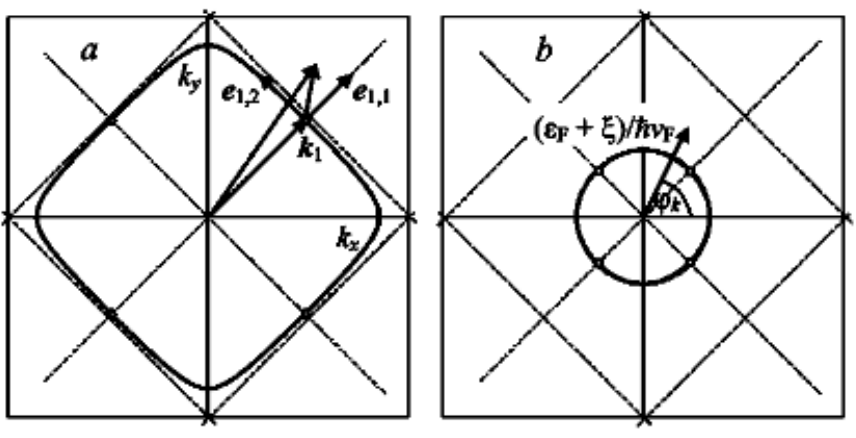

Figure 3.1: Fermi surfaces for a $d$-wave superconductor: (a) "squarelike" geometry at closeness to half filling $\left(\varepsilon_{\mathrm{F}} / W=0.475\right)$. The local axes $e_{1,1}$ and $e_{1,2}$ are shown explicitly for the wave vector $\mathbf{k}$ near the nodal point $\mathbf{k}_{1}$; (b) "circularlike" geometry at lower doping $\left(\varepsilon_{\mathrm{F}} / W=0.125\right)$, almost indistinguishable from exact circle (thin dashed line).

and $\mathbf{e}_{i, 2}=\left(\mathbf{e}_{i+1,1}-\mathbf{e}_{i-1,1}\right) / 2$ [Fig. 3.1(a)], and then approximates the spectrum components as,

$$
\xi_{\mathrm{k}}=\hbar v_{\mathrm{F}} k_{1}, \quad \Delta_{\mathrm{k}}=\hbar v_{2} k_{2}
$$

with two characteristic velocities $v_{2} \ll v_{\mathrm{F}}$. However, this "square" geometry is a good approximation only for low quasiparticle energies $\left(E_{\mathbf{k}} \ll \Delta=\max _{\mathbf{k}}\left|\Delta_{\mathbf{k}}\right|\right)$ [except for the special case of closeness to half filling as shown in Fig. 3.1(a)]. Generally, when treating higher energies $E_{\mathbf{k}} \gtrsim \Delta$, one must consider a real Fermi surface geometry that in many cases is close to circular, [125, 126, 127, 128] and within the adopted approximation this relates to a small enough Fermi surface $\varepsilon_{\mathrm{F}} \ll W$ [Fig. 3.1(b)]. In this case, a more adequate parametrization of spectrum is obtained with $\xi_{\mathrm{k}}=\hbar v_{\mathrm{F}}\left(k-k_{\mathrm{F}}\right)$ and $\Delta_{\mathrm{k}}=\Delta \cos 2 \varphi_{k} \theta\left(\varepsilon_{\mathrm{D}}^{2}-\xi_{\mathrm{k}}^{2}\right)$ where $\varphi_{k}=\arctan \left(k_{y} / k_{x}\right)$ and the theta-function factor restricts SC pairing to the BCS shell of width $\varepsilon_{\mathrm{D}}$ (Debye energy) around $\varepsilon_{\mathrm{F}}$.

Most of the known treatments of impurity effects in doped and disordered $d$ wave $\mathrm{SC}$ systems, including a self-consistent $T$-matrix approximation (SCTMA), [129] were developed within pointlike perturbation models (though extended impurity perturbations were also studied by numerical treatment of Bogolyubovde Gennes equations on finite-size lattices [130]) and "square" geometry of Fermi surface.[114, 116] This paper includes more realistic features, either of the impurity perturbation (which can affect several equivalent neighbor sites to the impurity ion) and of the Fermi surface geometry (in a more adequate circular approximation) into analytic calculations using the Green's-function 
method. Apart from confirmation of the known results for pointlike centers, we search for new impurity effects, specific for extended perturbation

\section{Physical Description of the System}

We use the Nambu spinors $\Psi_{\mathbf{k}}^{\dagger}=\left(a_{\mathbf{k}, \uparrow}^{\dagger}, a_{-\mathbf{k}, \downarrow}\right)$ where $a_{\mathbf{k}, \sigma}^{\dagger}$ and $a_{\mathbf{k}, \sigma}$ are the Fermi operators for quasiparticles with wave vector $\mathbf{k}$ and spin $\sigma$ and the model Hamiltonian for disordered $d$-wave superconductor is

$$
\begin{gathered}
H=H_{0}+H_{i m p}, \\
H_{0}=\sum_{\mathbf{k}} \Psi_{\mathbf{k}}^{\dagger}\left(\xi_{\mathbf{k}} \widehat{\tau}_{3}-\Delta_{\mathbf{k}} \widehat{\tau}_{1}\right) \Psi_{\mathbf{k}}, \\
H_{i m p}=-\frac{1}{N} \sum_{\mathbf{k}, \mathbf{k}^{\prime}, \mathbf{p}} \mathrm{e}^{i\left(\mathbf{k}^{\prime}-\mathbf{k}\right) \mathbf{p}} \sum_{\delta} \mathrm{e}^{i\left(\mathbf{k}^{\prime}-\mathbf{k}\right) \delta} \Psi_{\mathbf{k}^{\prime}}^{\dagger} \widehat{V} \Psi_{\mathbf{k}} .
\end{gathered}
$$

In what follows we denote by hats the matrices in Nambu indices, e.g., the Pauli matrices $\widehat{\tau}_{i}$ and the matrix $\widehat{V}=V_{i m p} \widehat{\tau}_{3}$ which describes the quasiparticle scattering by extended (attractive) perturbation $V_{\text {imp }}$ around an impurity center p, over its near neighbors $\delta$ (Fig. 3.2). Formally, this perturbation only differs by the presence of $\widehat{\tau}_{3}$ factor from that considered in Ref. [124]. The concentration of randomly distributed centers $c=N^{-1} \sum_{\mathbf{p}} 1$ (where $N$ is the number of cells) is supposed small, $c \ll 1$.

We define the Green function (GF) matrices as:

$$
\widehat{G}_{\mathbf{k}, \mathbf{k}^{\prime}}(\varepsilon)=\left\langle\left\langle\widehat{\Psi}_{\mathbf{k}} \mid \widehat{\Psi}_{\mathbf{k}^{\prime}}^{\dagger}\right\rangle\right\rangle=i \int_{-\infty}^{0} e^{i(\varepsilon-i 0) t}\left\langle\left\{\widehat{\Psi}_{\mathbf{k}}(t), \widehat{\Psi}_{\mathbf{k}^{\prime}}^{\dagger}(0)\right\}\right\rangle d t
$$

where $\langle\ldots\rangle$ is the quantum-statistical average with Hamiltonian, Eq. (3.1), and $\{a(t), b(0)\}$ the anticommutator of Heisenberg operators. The energy $\varepsilon$ is referred to the chemical potenial $\mu$ (identified in what follows with the Fermi energy $\left.\varepsilon_{\mathrm{F}}\right)$. The observable characteristics follow from the averages of products of these operators (at given inverse temperature $\beta$ ), expressed through the corresponding GFs by the spectral theorem

$$
\langle a b\rangle=\int_{-\infty}^{\infty} \frac{d \varepsilon}{\mathrm{e}^{\beta \varepsilon}+1} \operatorname{Im}\langle\langle b \mid a\rangle\rangle_{\varepsilon} .
$$




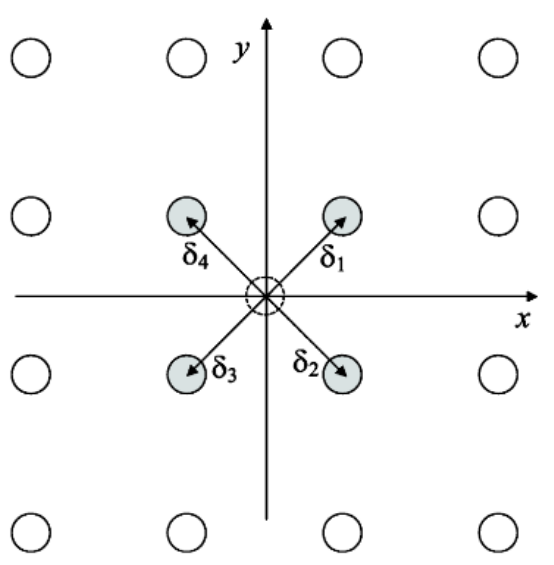

Figure 3.2: Extended perturbation over four nearest neighbor sites to the impurity ion (its projection onto the $\mathrm{CuO}_{2}$ plane is shown by the dashed circle at the origin).

For the disordered system with the Hamiltonian, Eq. (3.1), we calculate GFs from the basic equation of motion,

$$
\widehat{G}_{\mathbf{k}, \mathbf{k}^{\prime}}=\widehat{G}_{\mathbf{k}}^{0} \delta_{\mathbf{k}, \mathbf{k}^{\prime}}-\frac{1}{N} \sum_{\mathbf{k}^{\prime \prime}, \mathbf{p}, j} \mathrm{e}^{i\left(\mathbf{k}-\mathbf{k}^{\prime \prime}\right) \mathbf{p}} \alpha_{j \mathbf{k}} \alpha_{j \mathbf{k}^{\prime \prime}} \widehat{G}_{\mathbf{k}}^{0} \widehat{V} \widehat{G}_{\mathbf{k}^{\prime \prime}, \mathbf{k}^{\prime}}
$$

where $\widehat{G}_{\mathbf{k}}^{0}=\left(\varepsilon-\xi_{\mathbf{k}} \widehat{\tau}_{3}-\Delta_{\mathbf{k}} \widehat{\tau}_{1}\right)^{-1}$, and we expanded the structural function for impurity scattering in Eq. (3.1) as: $\sum_{\delta} e^{i\left(\mathbf{k}^{\prime}-\mathbf{k}\right) \delta}=\sum_{j=1}^{4} \alpha_{j \mathbf{k}} \alpha_{j \mathbf{k}^{\prime}}$. The functions

$$
\begin{aligned}
\alpha_{1, \mathbf{k}} & =2 \cos \frac{a k_{x}}{2} \cos \frac{a k_{y}}{2}, & \alpha_{2, \mathbf{k}} & =2 \cos \frac{a k_{x}}{2} \sin \frac{a k_{y}}{2}, \\
\alpha_{3, \mathbf{k}} & =2 \sin \frac{a k_{x}}{2} \cos \frac{a k_{y}}{2}, & \alpha_{4, \mathbf{k}} & =2 \sin \frac{a k_{x}}{2} \sin \frac{a k_{y}}{2},
\end{aligned}
$$

realize irreducible representations of the $C_{4}$ point group $(j=1$ being related to $A$-, $j=2,3$ to $E$-, and $j=4$ to $B$-representations [131]) and thus satisfy the orthogonality condition

$$
\frac{1}{N} \sum_{\mathbf{k}} \alpha_{j, \mathbf{k}} \alpha_{j^{\prime} \mathbf{k}}=\delta_{j j^{\prime}}
$$


The impurity effects on quasiparticle spectrum are then naturally classified along these representations, like the known results for magnetic impurities in ferro- and antiferromagnetic crystals. [132, 133]

\section{T-Matrix Solutions for the Green's Functions}

The orthogonality of the $\alpha_{j, \mathbf{k}}$ functions implies that in the general solution to Eq. 3.4

$$
\widehat{G}_{\mathbf{k}}=\left[\left(\widehat{G}_{\mathbf{k}}^{0}\right)^{-1}-\widehat{\Sigma}_{\mathbf{k}}\right]^{-1}
$$

has the self-energy matrix additive in these representations: $\widehat{\Sigma}_{\mathbf{k}}=\sum_{j} \widehat{\Sigma}_{j \mathbf{k}}$. Each partial term in the latter sum can be given by a specific group expansion (GE), like those known for point-like impurity perturbations in normal [134] or superconducting $[114,135]$ systems and also for extended perturbations in magnetic systems [132],

$$
\begin{aligned}
\widehat{\Sigma}_{j \mathbf{k}}= & -c \widehat{T}_{j}\left\{1-c \widehat{A}_{j}-c \widehat{A}_{j}^{2}+c \sum_{\mathbf{n} \neq 0}\left[\widehat{A}_{j}^{3}(n) \mathrm{e}^{-i \mathbf{k n}}+\widehat{A}_{j}^{4}(n)\right]\right. \\
& \left.\times\left[1-\widehat{A}_{j}^{2}(n)\right]^{-1}+\ldots\right\}
\end{aligned}
$$

Here $\widehat{T}_{j}=\widehat{V}\left(1+\widehat{V} \widehat{G}_{j}\right)^{-1}$ is the (renormalized) partial $T$-matrix, and the matrices $\widehat{A}_{j}(n)$ represent indirect interactions (in $j$ th symmetry channel) between scatterers at sites 0 and $\mathbf{n}$,

$$
\begin{aligned}
\widehat{A}_{j}(n) & =-\widehat{G}_{j}(n) \widehat{T}_{j}, & \widehat{G}_{j}(n) & =\frac{1}{N} \sum_{\mathbf{k}} \mathrm{e}^{i \mathbf{k n}} \alpha_{j, \mathbf{k}}^{2} \widehat{G}_{\mathbf{k}}, \\
\widehat{A}_{j} & =\widehat{G}_{j} \widehat{T}_{j}, & \widehat{G}_{j} & =\widehat{G}_{j}(0) .
\end{aligned}
$$

The sum $\sum_{\mathbf{n} \neq 0}$ in Eq. (3.7) describes all the processes involving pairs of impurities, it implies averaging in random impurity configurations, and hence runs over all the lattice sites $\mathbf{n}$. The omitted terms are for triples and more of impurities. This defines a generalization of the GE approach for extended impurity centers in superconductors. If the series in the brackets is restricted to its first term, the self-energy matrix $\widehat{\Sigma}_{\mathbf{k}}$ becomes independent of $\mathbf{k}$, 


$$
\widehat{\Sigma}_{\mathbf{k}} \rightarrow \widehat{\Sigma}=-c \sum_{j} \widehat{T}_{j}
$$

Then, for small enough concentration of impurities, the renormalization of $T$-matrices can be neglected and we arrive at

$$
\widehat{T}_{j} \rightarrow \widehat{T}_{j}^{0}=\widehat{V}\left(1+\widehat{G}_{j}^{0} \widehat{V}\right)^{-1}, \quad \widehat{G}_{j}^{0}=\frac{1}{N} \sum_{\mathbf{k}} \alpha_{j, \mathbf{k}}^{2} \widehat{G}_{\mathbf{k}}^{0}
$$

The matrix functions $\widehat{G}_{j}^{0}$ can be expanded in the basis of Pauli matrices

$$
\widehat{G}_{j}^{0}=\rho_{0}\left(g_{j 0}+g_{j 1} \widehat{\tau}_{1}-g_{j 3} \widehat{\tau}_{3}\right)
$$

where $\rho_{0}=4 /(\pi W)$ is the constant DOS in a 2D normal system [the absence of $\widehat{\tau}_{2}$ component in Eq. (3.10) is related to the fact that the gap function $\Delta_{\mathbf{k}}$ is chosen real]. Passing in Eq. (3.9) from summation in $\mathbf{k}$ to integration in "polar" coordinates $\xi_{\mathbf{k}}=\xi$ and $\varphi_{\mathbf{k}}=\varphi$ [Fig. 3.1(b)] according to the rule,

$$
\frac{1}{N} \sum_{\mathbf{k}} f_{\mathbf{k}} \approx \frac{\rho_{0}}{4 \pi} \int_{-\mu}^{2 / \rho_{0}-\mu} d \xi \int_{0}^{2 \pi} d \varphi f(\xi, \varphi)
$$

we calculate the dimensionless coefficient functions $g_{j i}$. Some of them are zero by the symmetry reasons: $g_{11}=g_{41}=0$. The rest can be approximated as

$$
\begin{gathered}
g_{j 0} \approx \overline{\alpha_{j}^{2}} g_{0}, \quad g_{j 3} \approx \overline{\alpha_{j}^{2}} g_{3}, \\
g_{21}=-g_{31} \approx \overline{\alpha_{2}^{2}} g_{1} .
\end{gathered}
$$

Here $\overline{\alpha_{j}^{2}}$ are the average values of $\alpha_{j \mathbf{k}}^{2}$ over the Fermi surface: $\overline{\alpha_{1}^{2}} \approx 4(1-\omega)$, $\overline{\alpha_{2,3}^{2}} \approx 4 \omega, \overline{\alpha_{4}^{2}} \approx 2 \omega^{2}$, where the band occupation parameter $\omega=\mu / W$ is supposed small, in concordance with the chosen circular geometry. The functions $g_{0}$ and $g_{1}$ are known from the studies of pointlike perturbations: $[136,137]$

$$
\begin{aligned}
g_{0}(\varepsilon) & =\frac{\varepsilon}{4 \pi} \int_{-\mu}^{2 / \rho_{0}-\mu} d \xi \int_{0}^{2 \pi} \frac{d \varphi}{\varepsilon^{2}-\xi^{2}-\Delta^{2} \cos ^{2} 2 \varphi} \\
& \approx \varepsilon\left[1 / \tilde{\mu}-\mathrm{F}_{1}\left(1-\varepsilon^{2} / \Delta^{2}\right) / \Delta\right],
\end{aligned}
$$




$$
\begin{aligned}
& g_{1}(\varepsilon)= \frac{\Delta}{2 \pi} \int_{0}^{\varepsilon_{\mathrm{D}}} d \xi \int_{0}^{2 \pi} \frac{\cos ^{2} 2 \varphi d \varphi}{\varepsilon^{2}-\xi^{2}-\Delta^{2} \cos ^{2} 2 \varphi} \\
& \approx \Delta / \varepsilon_{\mathrm{D}}+2\left[\mathrm{~F}_{2}\left(1-\varepsilon^{2} / \Delta^{2}\right)+\left(\varepsilon^{2} / \Delta^{2}\right)\right. \\
&\left.\times \mathrm{F}_{1}\left(1-\varepsilon^{2} / \Delta^{2}\right)\right]
\end{aligned}
$$

They include $\tilde{\mu}=\mu(1-2 \omega / \pi) \approx \mu$ and the functions $\mathrm{F}_{1}(\mathrm{z})=\mathrm{K}(1 / \mathrm{z}) / \sqrt{\mathrm{z}}$ and $\mathrm{F}_{2}(\mathrm{z})=\sqrt{\mathrm{z}} \mathrm{E}(1 / \mathrm{z})$ with full elliptic integrals of first and second kind $\mathrm{K}$ and $\mathrm{E}$, having similar behavior with the elementary functions obtained within square geometry. [116] In the same similarity, the function $g_{3}=(4 \pi)^{-1} \int_{-\mu}^{2 / \rho_{0}-\mu} \xi d \xi \int_{0}^{2 \pi} d \varphi /\left(\varepsilon^{2}-\right.$ $\left.\xi^{2}-\Delta^{2} \cos ^{2} 2 \varphi\right)$ is practically constant: $g_{3} \approx \ln \sqrt{\pi /(2 \omega)-1}$, within the relevant energy range $|\varepsilon| \ll W, \mu$.

Using these results, we readily calculate the partial $T$ matrices, Eq. (3.9). The most important contribution to $\widehat{\Sigma}$ comes from the $j=1$ term $(A$ representation):

$$
\widehat{T}_{1}^{0}=\frac{v_{A}}{\overline{\alpha_{1}^{2}} \rho_{0}} \frac{v_{A} g_{0}-\widehat{\tau}_{3}}{D_{A}}
$$

where $v_{A}=\overline{\alpha_{1}^{2}} V_{i m p} \rho_{0} /\left(1-\overline{\alpha_{1}^{2}} V_{i m p} \rho_{0} g_{3}\right)$ is the dimensionless perturbation parameter in the $A$ channel, and $D_{A}(\varepsilon)=1-v_{A}^{2} g_{0}^{2}(\varepsilon)$ is the energy dependent denominator. In particular, it can produce a low energy resonance at $\varepsilon=\varepsilon_{\text {res }}$ such that $\operatorname{Re} D_{A}\left(\varepsilon_{r e s}\right)=0$, analogous to the above mentioned resonance from pointlike impurity center. This requires that $v_{A}$ exceeds some critical value $\approx 2 / \pi$. The resonance can approach the Fermi level, $\varepsilon_{r e s} \rightarrow 0$, only in the unitary limit for perturbation, $v_{A} \rightarrow 0$, unlike the case of Ref. [124] where this can happen for a finite spin-dependent perturbation.

The contributions from $j=2,3$ (E-representation) are

$$
\widehat{T}_{2,3}^{0}=\frac{v_{E}}{\overline{\alpha_{2}^{2}} \rho_{0}} \frac{v_{E}\left(g_{0} \mp g_{1} \widehat{\tau}_{1}\right)-\widehat{\tau}_{3}}{D_{E}},
$$

with the respective perturbation parameter $v_{E}=\overline{\alpha_{2}^{2}} V_{i m p} \rho_{0} /\left(1-\overline{\alpha_{2}^{2}} V_{i m p} \rho_{0} g_{3}\right)$ and denominator $D_{E}=1-v_{E}^{2}\left(g_{0}^{2}-g_{1}^{2}\right)$. It is less probable to have a resonance effect in this channel at low occupation $\omega \ll 1$, since (i) the parameter $v_{E}$ is reduced versus the $A$-channel value, and (ii) there is a competition between $\operatorname{Re} g_{0}^{2}$ and $\operatorname{Re} g_{1}^{2}$ in the denominator $D_{E}$. 
The $B$-channel contribution $(j=4)$ has the same structure as the $A$-channel term, Eq. (3.14), but with $v_{A}$ replaced by a strongly reduced value $v_{B}=$ $\overline{\alpha_{4}^{2}} V_{i m p} \rho_{0} /\left(1-\overline{\alpha_{4}^{2}} V_{i m p} \rho_{0} g_{3}\right)$; hence it turns even less important than the $E$ channel terms.

\section{Perturbation of Observable Values}

Now we are in a position to describe the perturbation of basic observable characteristics of SC system by extended impurity centers. Thus, the global DOS is defined by the momentum diagonal GFs, $\rho(\varepsilon)=(\pi N)^{-1} \sum_{\mathbf{k}} \operatorname{Im} \operatorname{Tr} \widehat{G}_{\mathbf{k}}$, and, using Eqs. (3.8), (3.14), (3.15) Eq. (3.6), it is obtained as

$$
\rho(\varepsilon)=\frac{\rho_{0}}{\pi} \operatorname{Im} g_{0}\left(\varepsilon-\Sigma_{0}\right),
$$

where the scalar self-energy

$$
\Sigma_{0}=\frac{c g_{0}(\varepsilon)}{\rho_{0}}\left(\frac{v_{A}^{2}}{\overline{\alpha_{1}^{2} D_{A}}}+\frac{2 v_{E}^{2}}{\overline{\alpha_{2}^{2}} D_{E}}+\frac{v_{B}^{2}}{\overline{\alpha_{4}^{2}} D_{B}}\right)
$$

includes the effects of extended impurity centers in all three channels. The result of direct calculation from Eq. 3.16 with use of Eq. 3.17 for the characteristic choice of parameters, $W=2 \mathrm{eV}, \mu=0.3 \mathrm{eV}, \varepsilon_{\mathrm{D}}=0.15 \mathrm{eV}, V_{i m p}=0.2$ $\mathrm{eV}$ (giving for particular channels: $v_{A} \approx 0.934, v_{E} \approx 0.088$, and $v_{B} \approx 0.006$ ), and $c=0.15$, is shown in Fig. 3.3. It is quite similar to the known results for point-like impurities, $[87,115]$ showing a reduction of the sharp coherence peak at $\varepsilon=\Delta$ and emergence of a relatively broad low-energy resonance at $\varepsilon_{\text {res }}$ (shown by the arrow), mainly due to the $A$-channel effect, but, additionally, there are small "antiresonance" effects from the $E$-channel (insets to Fig. 3.3 ), at $\varepsilon \approx \Delta$ and at some high enough energy ( $\sim 70 \Delta$ in this case). These $E$-channel features (the high-energy one even probably nonexisting) should not have any sizeable effect on the system thermodynamics.

The local density of states (LDOS) on $\mathbf{n t h}$ site is expressed in terms of GFs as $\rho_{\mathbf{n}}(\varepsilon)=(\pi N)^{-1} \sum_{\mathbf{k}, \mathbf{k}^{\prime}} \operatorname{Im} \operatorname{Tr} \mathrm{e}^{i\left(\mathbf{k}-\mathbf{k}^{\prime}\right) \cdot \mathbf{n}} \widehat{G}_{\mathbf{k}, \mathbf{k}^{\prime}}$ and its variation $\delta \rho_{\mathbf{n}}(\varepsilon)=\rho_{\mathbf{n}}(\varepsilon)-$ $\rho(\varepsilon)$, compared to the mean value $\rho(\varepsilon)=N^{-1} \sum_{\mathbf{n}} \rho_{\mathbf{n}}(\varepsilon)$ (identical to the global DOS), is only given by the momentum-nondiagonal GFs,

$$
\delta \rho_{\mathbf{n}}(\varepsilon)=\frac{1}{\pi N} \sum_{\mathbf{k}, \mathbf{k}^{\prime} \neq \mathbf{k}} \operatorname{Im} \operatorname{Tr} \mathrm{e}^{i\left(\mathbf{k}-\mathbf{k}^{\prime}\right) \cdot \mathbf{n}} \widehat{G}_{\mathbf{k}, \mathbf{k}^{\prime}}
$$




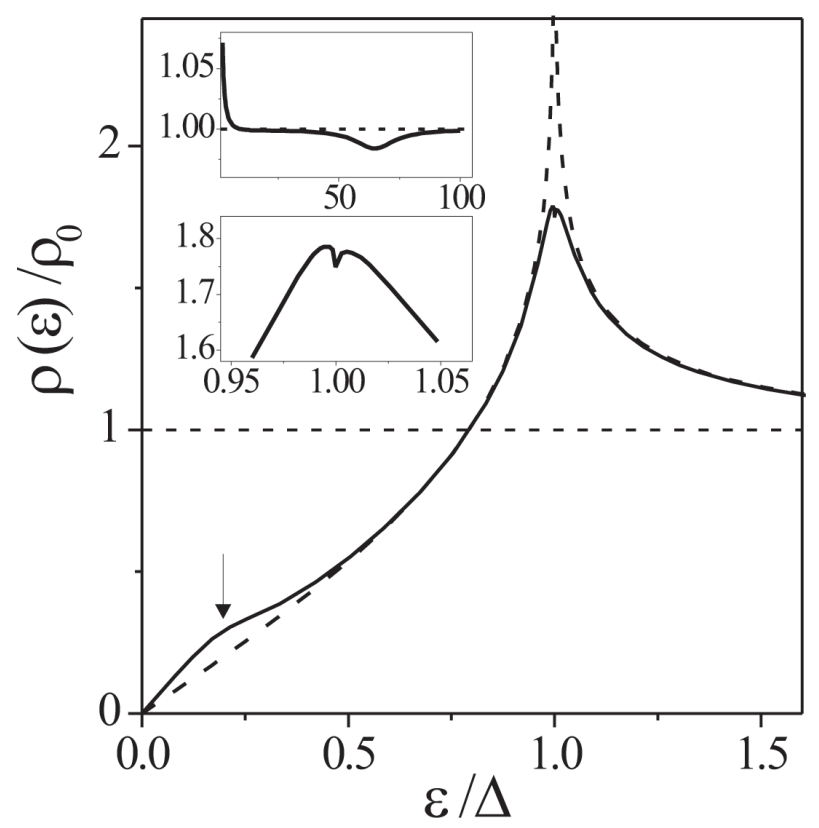

Figure 3.3: Density of states in the $d$-wave superconductor with extended impurity centers (the solid line), for the choice of parameters $W=2 \mathrm{eV}, \mu=0.3 \mathrm{eV}, \varepsilon_{\mathrm{D}}=0.15 \mathrm{eV}$, $V_{i m p}=0.2 \mathrm{eV}, c=0.15$. The arrow indicates the low-energy resonance by the $A$-channel impurity effect and the dashed line represents the pure $d$-wave DOS. Insets: weak $E$-channel "antiresonances" at high energies (upper panel) and near the gap edge (lower panel). 


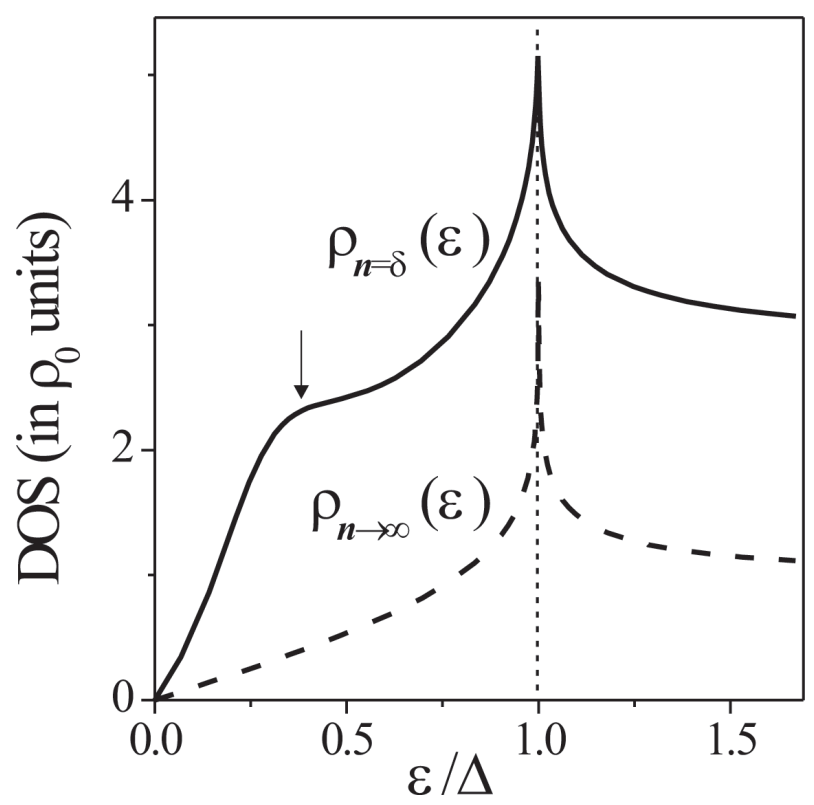

Figure 3.4: Local density of states on the nearest-neighbor site to an extended impurity center, for the same choice of parameters as in Fig. 3.3 (but supposing $c \rightarrow 0$ ). Note an overall enhancement of electronic density compared to remote sites from impurity (dashed line) and a much stronger effect of the low-energy resonance (the arrow).

These functions are easily calculated for the simplest case of a single impurity center at $\mathbf{p}=0$,

$$
\widehat{G}_{\mathbf{k}, \mathbf{k}^{\prime}}=\frac{1}{N} \sum_{j} \alpha_{j, \mathbf{k}} \widehat{G}_{\mathbf{k}}^{0} \widehat{T}_{j}^{0} \widehat{G}_{\mathbf{k}^{\prime}}^{0} \alpha_{j, \mathbf{k}^{\prime}}
$$

describing a finite effect on the local characteristics near the impurity. Thus, the quantity $\delta \rho_{\mathbf{n}}$ attains its maximum value at $\mathbf{n}=\delta$, the nearest-neighbor sites to the impurity. Using Eq. (3.19) and the orthogonality relations, we expand this value in a sum,

$$
\begin{aligned}
\delta \rho_{\mathbf{n}=\delta}(\varepsilon)=\quad & \frac{1}{\pi N^{2}} \sum_{\mathbf{k}, \mathbf{k}^{\prime}, j} \operatorname{Im} \operatorname{Tr} \mathrm{e}^{i \mathbf{k} \cdot \delta} \alpha_{j, \mathbf{k}} \widehat{G}_{\mathbf{k}}^{0} \widehat{T}_{j}^{0} \widehat{G}_{\mathbf{k}^{\prime}}^{0} \alpha_{j, \mathbf{k}^{\prime}} \mathrm{e}^{-i \mathbf{k}^{\prime} \cdot \delta} \\
= & \frac{1}{\pi} \sum_{j} \operatorname{Im} \operatorname{Tr} \widehat{G}_{j}^{0} \widehat{T}_{j}^{0} \widehat{G}_{j}^{0}
\end{aligned}
$$


and present the overall maximum LDOS as

$$
\rho_{\mathbf{n}=\delta}(\varepsilon)=\frac{2 \rho_{0}}{\pi} \operatorname{Im}\left[g_{0}(\varepsilon)\left(1+\frac{v_{A} N_{A}}{\overline{\alpha_{1}^{2}} D_{A}}+2 \frac{v_{E} N_{E}}{\overline{\alpha_{2}^{2}} D_{E}}+\frac{v_{B} N_{B}}{\overline{\alpha_{4}^{2}} D_{B}}\right)\right] .
$$

Similar to the lienar in $c$, Eq. (3.17), for global DOS and the case of Ref. [124] for LDOS, the resonance contribution to Eq. (3.20) comes from the $A$ channel with the numerator $N_{A}=2 g_{3}+v_{A}\left(g_{0}^{2}+g_{3}^{2}\right)$, while other channels with $N_{E}=2 g_{3}+v_{E}\left(g_{0}^{2}-g_{1}^{2}-g_{3}^{2}\right)$ and $N_{B}=2 g_{3}+v_{B}\left(g_{0}^{2}+g_{3}^{2}\right)$ mainly contribute to renormalization of the pure $d$-wave $\operatorname{DOS} \rho_{d}(\varepsilon)=2 / \pi \operatorname{Im} g_{0}(\varepsilon)$.

The behavior calculated from Eq. (3.20) of LDOS on nearest-neighbor sites to the impurity is shown by solid line in Fig. 3.4. It displays a low energy resonance (the arrow), much more pronounced than that in the global DOS, Fig. 3.3, and an overall enhancement compared to the LDOS curve for remote sites from impurity $\rho_{\mathbf{n} \rightarrow \infty}=\rho_{d}$ (the dashed line).

The latter effect is related to the local attraction by impurity and, of course, the overall particle number is conserved: $N^{1} \sum_{\mathbf{n}} \int \rho_{\mathbf{n}}(\varepsilon) d \varepsilon=1$. The curves in Fig. 3.4 can be compared with the direct experimental measurements of differential conductance through the STM tip positioned close to and far from an impurity center. [117]

In a similar manner, the local perturbation of SC order parameter can be considered. The local $d$-wave SC order in the unit cell containing the impurity (Fig. 3.2) is given by the average $\Delta_{32}=2 V\left\langle a_{\delta_{3}, \downarrow} a_{\delta_{2}, \uparrow}\right\rangle,[124]$ where $V$ is the SC coupling constant and site operators $a_{\mathbf{n}, \sigma}$ are expressed through band operators: $a_{\mathbf{n}, \sigma}=N^{-1 / 2} \sum_{\mathbf{k}} \mathrm{e}^{i \mathbf{k} \cdot \mathbf{n}} a_{\mathbf{k}, \sigma}$. For $V_{\mathrm{imp}}=0$, this average coincides with the uniform gap parameter

$$
\begin{gathered}
\Delta=\frac{2 V}{N} \sum_{\mathbf{k}} \mathrm{e}^{i \mathbf{k} \cdot\left(\delta_{2}-\delta_{3}\right)}\left\langle a_{-\mathbf{k}, \downarrow} a_{\mathbf{k}, \uparrow}\right\rangle \\
=\frac{4 \lambda \Delta}{\pi} \int_{0}^{\varepsilon_{\mathrm{D}} / \Delta}\left[\mathrm{F}_{2}\left(1+\mathrm{x}^{2}\right)-\mathrm{F}_{1}\left(1+\mathrm{x}^{2}\right)\right] d x
\end{gathered}
$$

the latter expression (with the dimensionless $d$-wave coupling constant $\lambda=$ $\left.V \rho_{0} \omega\right)$ being obtained from Eqs. (3.3) and (3.13). The integral in Eq. (3.21) behaves as a logarithm: $\int_{0}^{a}\left[\mathrm{~F}_{2}\left(1+x^{2}\right)-\mathrm{F}_{1}\left(1+x^{2}\right)\right] d x \approx(\pi / 4) \ln (2.428 a)$ at $a \gg 1$, thus providing $\Delta \approx 2.428 \varepsilon_{\mathrm{D}} \exp (-1 / \lambda)$. 


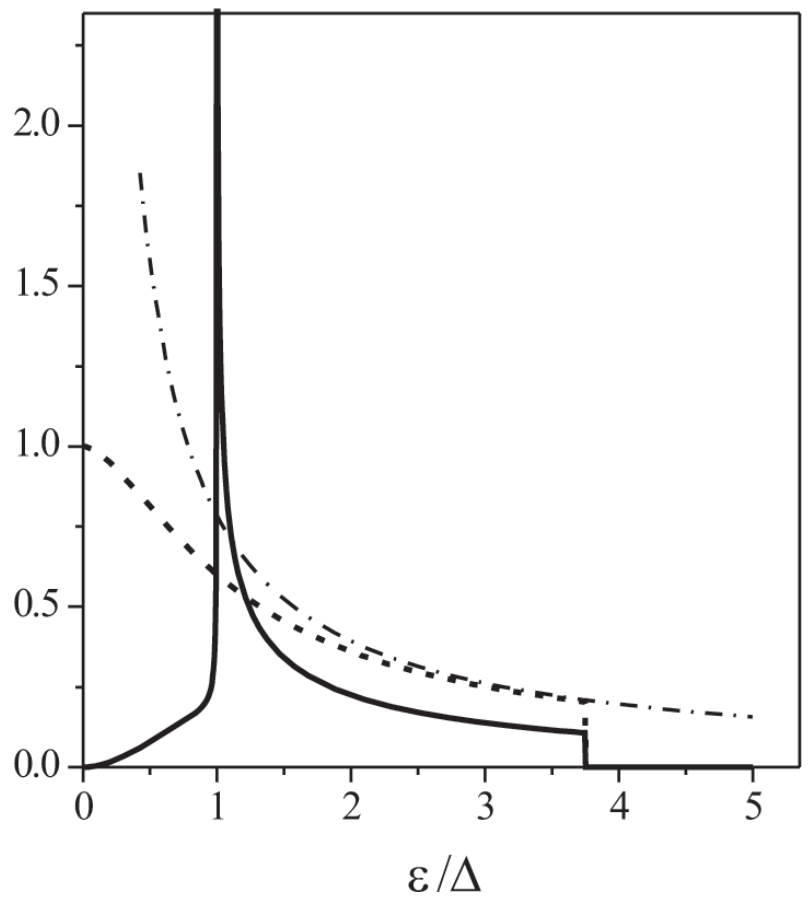

Figure 3.5: Dimensionless function $F(\varepsilon / \Delta)$ (solid line) used in Eq. (3.24) to calculate the suppression parameter $\eta_{\text {sup }}$, at the same choice of parameters as in Fig. 3.4, compared to the integrand in the uniform gap equation, Eq. (3.21) (dashed line) and its asymptotics $\pi \Delta /(4 \varepsilon)$ (dash-dotted line).

But for $V_{\mathrm{imp}} \neq 0$ this value is locally suppressed. The suppression is characterized by the dimensionless parameter $\eta_{\text {sup }}=1-\Delta_{32} / \Delta$, confined between 0 (pure SC) and 1 (complete local suppression of SC order),[124] and it only results from nondiagonal GFs,

$$
\begin{gathered}
\eta_{\text {sup }}=\frac{2 V}{N \Delta} \sum_{\mathbf{k}, \mathbf{k}^{\prime} \neq \mathbf{k}}\left\langle a_{-\mathbf{k}, \downarrow} a_{\mathbf{k}^{\prime}, \uparrow}\right\rangle \mathrm{e}^{i\left(\mathbf{k} \cdot \delta_{2}-\mathbf{k}^{\prime} \cdot \delta_{3}\right)}= \\
=\frac{V}{4 \pi \Delta} \sum_{j}(-1)^{j} \int_{-\infty}^{0} d \varepsilon \operatorname{Im} \operatorname{Tr} \widehat{G}_{j}^{0} \widehat{T}_{j}^{0} \widehat{G}_{j}^{0} \widehat{T}_{1} .
\end{gathered}
$$

Using here Eqs. (3.19) and (3.10) leads to the expression, 


$$
\eta_{\text {sup }}=\frac{4 \lambda}{\pi} \int_{0}^{\varepsilon_{\mathrm{D}} / \Delta} F(x) d x
$$

where only the $E$-channel terms contribute to the dimensionless function

$$
F(x)=-\frac{v_{E}}{2} \operatorname{Im} \frac{g_{1}(x \Delta) N_{E}}{D_{E}}
$$

[compared in Fig. 3.5 with the integrand for the uniform gap equation, Eq. (3.21)]. Numeric analysis of this expression for the above chosen perturbation parameters results in $\eta_{\text {sup }} \approx 0.47$. This is slightly smaller than the respective value for pointlike impurity in $d$-wave system:[87] $\eta_{\text {sup }}=1 /\left(1+v^{2}\right)$ (assuming $v$ equal to $v_{A}$ ), and about twice weaker than almost complete suppression in the case of spin-dependent perturbation of the same dimensionless magnitude. In the latter case, the analogous integrand function (as follows from Fig. 2 in Ref. [124])is close to the dashed line in Fig. 3.5. Those relations confirm the general Abrikosov-Gor'kov conclusion [138] on the pair-breaking effects by impurities in superconductors, irrespective of their spatial extension.

\section{Self-Consistent Generalization}

The above given analysis corresponds to the simplest restriction of the group series, Eq. (3.7), for self-energy to its first, single-impurity term with use of unperturbed GFs. The resulting linear approximation in impurity concentration $\widehat{\Sigma}=-c \sum_{j} \widehat{T}_{j}^{0}$, is only justified when this concentration is low enough $c \ll c_{0}$, where $c_{0} \sim \rho_{0} \varepsilon_{0}$ is related to the characteristic energy scale $\varepsilon_{0}$ for impurity perturbation (in this case $\varepsilon_{0} \sim \varepsilon_{\text {res }}$ ). At higher concentrations, $c>c_{0}$, when perturbations from different impurity centers effectively overlap. The simplest way to take account of these collective impurity effects is provided by replacement of $\widehat{\Sigma}$ by its self-consistent analogue $\widehat{\Sigma}^{(s c)}=-c \sum_{j} T_{j}^{(s c)}$, in the spirit of well-known SCTMA method. [116, 118, 139] It was shown for the case of pointlike impurity perturbation, [116] that effects of such self-consistency are most essential at the lowest excitation energies, $\varepsilon \ll \Delta$. In view of the similarity in the system response to pointlike and extended perturbations and of the predominant role of the $A$-channel at low energies, we can restrict the self-consistency of $T$-matrix only to its $A$-channel term. Then Eqs. (3.6) and (3.8) are modified to 


$$
\widehat{G}_{\mathbf{k}}^{(s c)}=\left[\left(\widehat{G}_{\mathbf{k}}^{0}\right)^{-1}+c \sum_{j=2}^{4} \widehat{T}_{j}+c \widehat{T}_{1}^{(s c)}\right]^{-1},
$$

including the self-consistent $A$-channel $T$-matrix:

$$
\widehat{T}_{1}^{(s c)}=\widehat{V}\left[1+\widehat{G}_{1}^{(s c)} \widehat{V}\right]^{-1} .
$$

As in Eq. (3.10), the self-consistent GF matrix $\widehat{G}_{1}^{(s c)}=N^{-1} \sum_{\mathbf{k}} \alpha_{1 \mathbf{k}}^{2} \widehat{G}_{\mathbf{k}}^{(s c)}$ can be parametrized in Pauli matrices,

$$
\widehat{G}_{1}^{0}=\rho_{0} \overline{\alpha_{1}^{2}}\left(g-g_{3} \widehat{\tau}_{3}\right)
$$

Then, using Eq. (3.15) in Eq. (3.24), we readily conclude that $\widehat{\Sigma}^{(s c)}$ is diagonal in Nambu indices; that is, within the considered SCTMA for extended impurity centers, the scattering by dopants does not influence the $d$-wave order parameter the same as for pointlike centers.[114] Hence, the self-consistency should be achieved only for the scalar function $g=(2 N)^{-1} \sum_{\mathbf{k}} \operatorname{Tr} \widehat{G}_{\mathbf{k}}^{(s c)}$ through the equation

$$
g(\varepsilon)=g_{0}\left(\varepsilon-\Sigma_{0}^{(s c)}(\varepsilon)\right),
$$

where the scalar self-consistent self-energy

$$
\Sigma_{0}^{(s c)}(\varepsilon)=\frac{c g(\varepsilon)}{\rho_{0}}\left(\frac{v_{A}^{2}}{\overline{\alpha_{1}^{2}} D_{A}^{(s c)}}+\frac{2 v_{E}^{2}}{\overline{\alpha_{2}^{2}} D_{E}}+\frac{v_{B}^{2}}{\overline{\alpha_{4}^{2}} D_{B}}\right),
$$

includes the self-consistent denominator $D_{A}^{(s c)}(\varepsilon)=1-v_{A}^{2} g^{2}(\varepsilon)$. Then, passing to dimensionless energy $x=\varepsilon / \Delta$ and denoting

$$
\frac{\Sigma_{0}^{(s c]}}{\Delta}=\sigma(g)=\alpha g\left(\frac{\beta}{1-\alpha^{2} g^{2}}+\beta^{\prime}\right)
$$

with $\alpha=v_{A}, \beta=c v_{A} /\left(\overline{\alpha_{1}^{2}} \rho_{0} \Delta\right), \beta^{\prime}=c\left[2 v_{E}^{2} /\left(\overline{\alpha_{2}^{2}} D_{E}\right)+v_{B}^{2} /\left(\overline{\alpha_{4}^{2}} D_{B}\right)\right] /\left(v_{A} \rho_{0} \Delta\right)$, and $\gamma=\Delta /[\mu(1-2 \omega / \pi)]$, we arrive at the self-consistency equation for $g=g(x)$ as 


$$
g=(x-\sigma)\left\{\gamma-\frac{1}{\sqrt{1-(x-\sigma)^{2}}} \mathrm{~K}\left[\frac{1}{1-(x-\sigma)^{2}}\right]\right\}
$$

with $\sigma=\sigma(g)$ defined by Eq. (3.27). This equation is quite similar to that reported for pointlike impurity centers and squarelike geometry, $[114,116]$ differing only by the appearance of additional term $\beta^{\prime}$ in Eq. (3.27). In the same way, Eq. (3.28) admits two types of solutions in the energy range of principal interest $x \rightarrow 0$. One of them, $g=g^{(1)}(x)$, tends in this limit to a finite imaginary value, $g^{(1)}(x \rightarrow 0) \rightarrow i \gamma_{0}$, defined by the equation

$$
1=-\alpha f\left(\gamma_{0}\right)\left\{\gamma-\frac{1}{\sqrt{1+\alpha^{2} \gamma_{0}^{2} f^{2}\left(\gamma_{0}\right)}} \mathrm{K}\left[\frac{1}{1+\alpha^{2} \gamma_{0}^{2} f^{2}\left(\gamma_{0}\right)}\right]\right\},
$$

with $f\left(\gamma_{0}\right)=\beta^{\prime}+\beta /\left(1+\alpha^{2} \gamma_{0}^{2}\right)$. Another solution, $g=g^{(2)}(x)$, is vanishing in this limit: $g^{(2)}(x \rightarrow 0) \rightarrow 0$, so that all the denominators $D$ in Eq. (3.26) can be safely put equal to unity, simplifying Eq. (3.28) to

$$
g=\left(x-\alpha^{\prime} g\right)\left\{\gamma-\mathrm{K}\left[\frac{1}{1-\left(x-\alpha^{\prime} g\right)^{2}}\right]\right\},
$$

with $\alpha^{\prime}=\alpha\left(\beta+\beta^{\prime}\right)$. Its solution has the same logarithmic asymptotics at $x \rightarrow 0$,

$$
g(x) \approx \frac{x}{\alpha^{\prime}}\left[1-\frac{1}{\pi \alpha^{\prime} \ln \left(2 i \pi \alpha^{\prime} / x\right)}\right],
$$

as found in Refs. [114] and [116]. Also the conclusion as to which solution is valid in a specific energy range, $g^{(1)}$ far enough from and $g^{(2)}$ close to the Fermi level, [137] remains true in the present situation. Thus the DOS $\rho(\varepsilon)$ at $\varepsilon \ll \varepsilon_{\text {res }}$ should be suppressed as

$$
\rho(\varepsilon) \approx \rho_{0} \frac{\varepsilon}{2 \pi \Delta\left[\alpha^{\prime} \ln \left(2 \pi \alpha^{\prime} \Delta / \varepsilon\right)\right]^{2}}
$$

compared to its linear asymptotics $\rho \sim \rho_{0} \varepsilon / \Delta$ in the simple approximation of Eq. 3.16 shown in Fig. 3.3. 


\section{Conclusion}

The Green's function analysis is developed for the quasi-particle spectrum in a planar $d$-wave superconductor with finite concentration of impurity centers which perturb atomic energy levels on nearest-neighbor lattice sites. We demonstrate that in this case the general picture of spectrum restructuring is quite similar to that previously established for pointlike impurity perturbation, though some uniquely specific features due to the extended nature of the perturbation also appear. In particular, it is found that the effects on the quasiparticle DOS and on the SC order parameter result from different irreducible representations of the point symmetry group of the impurity center. Compared to the case of spin-dependent extended perturbation, the suppression of SC order near the considered impurity is much weaker. A generalization of the method of group expansions for quasiparticle self-energy is obtained for such extended impurity centers. The self-consistent procedure is developed, generalizing the known SCTMA formulation for pointlike centers, and a qualitative similarity with that case is demonstrated. These techniques can be also applied for other types of extended impurity centers where stronger effects of low-symmetry representations are not excluded. 

Chapter 4

\section{Specifics of impurity effects in ferropnictide superconductors}




\title{
Specifics of impurity effects in ferropnictide superconductors
}

\author{
Y. G. Pogorelov,,$^{1, *}$ M. C. Santos, ${ }^{2}$ and V. M. Loktev ${ }^{3}$ \\ ${ }^{1}$ IFIMUP-IN, Departamento de Física, Universidade do Porto, Porto, Portugal \\ ${ }^{2}$ Departamento de Física, Universidade de Coimbra, R. Larga, P-3004-535 Coimbra, Portugal \\ ${ }^{3}$ Bogolyubov Institute for Theoretical Physics, NAN of Ukraine, $14 \mathrm{~b}$ Metrologichna str., 03143 Kiev, Ukraine \\ (Received 26 May 2011; revised manuscript received 7 September 2011; published 7 October 2011) \\ Effects of impurities and disorder on quasiparticle spectrum in superconducting iron pnictides are considered. \\ The possibility of occurrence of localized energy levels due to impurities within the superconducting gap and the \\ related modification of band structure, including the emergence of narrow bands of extended quasiparticle states \\ near impurity levels, is analyzed. The evolution of a superconducting state with an impurity concentration is \\ traced and some specific effects of the modified quasiparticle spectrum on the superconducting order parameter \\ and other observable characteristics are discussed.
}

DOI: 10.1103/PhysRevB.84.144510

PACS number(s): 74.70.Xa, 74.62.Dh, 74.62.En

\section{INTRODUCTION}

The recent discovery of superconductivity (SC) with a rather high critical temperature in the family of doped ferropnictide compounds ${ }^{1,2}$ has motivated a great interest in these materials (see reviews in Refs. 3 and 4). Unlike the extensively studied cuprate family, ${ }^{5}$ which presents insulating properties in their initial undoped state, the undoped LaOFeAs compound is a semimetal. As established in previous physical and chemical studies (see, e.g., Refs. 6 and 7), this material has a layered structure, where the SC state is supported by the FeAs layer with a two-dimensional (2D) square lattice of $\mathrm{Fe}$ atoms and with As atoms located out of plane, above or below the centers of square cells (Fig. 1). Its electronic structure, relevant for constructing microscopic SC models, have been explored with high-resolution angle-resolved photoemission spectroscopy (ARPES) techniques. 8,9 Their results indicate the multiple connected structure of the Fermi surface, consisting of electron and hole pockets and an absence of nodes in both electron and hole spectrum gaps, ${ }^{8}$ suggesting that these systems display the so-called extended $s$-wave (also called $s_{ \pm}$wave) SC order, changing the order parameter sign between electron and hole segments. ${ }^{13}$

To study band structure, first-principles numeric calculations are commonly used, outlining the importance of $\mathrm{Fe}$ atomic $d$ orbitals. The calculations show that SC in these materials is associated with $\mathrm{Fe}$ atoms in the layer plane, represented in Fig. 1 by their orbitals and the related hopping amplitudes. The dominance of $\mathrm{Fe}$ atomic $3 d$ orbitals in the density of states (DOS) of the LaOFeAs compound near its Fermi surface was demonstrated by local density approximation (LDA) calculations. ${ }^{10-15}$ It was then concluded that multiorbital effects are important for the electronic excitation spectrum in the SC state, causing the formation of two spectrum gaps: by electron and hole pockets at the Fermi surface. To explain the observed SC properties, it is suggested that these materials may reveal an unconventional pairing mechanism, beyond the common electron-phonon scheme. ${ }^{16-19}$ In general, a total of five atomic orbitals for each iron in the LaOFeAs compound can be involved, however, ways to reduce this basis are sought, in order to simplify analytical and computational work. Some authors ${ }^{20,21}$ have suggested that it is sufficient to consider only $d_{x z}$ and $d_{y z}$ orbitals. Building such a minimal coupling model based on two orbitals, one is able to adjust the model parameters (energy hopping and chemical potential) to obtain a Fermi surface with the same topology as in the first-principles calculations of band structure. Even though it fails to reproduce some finer features of the electronic spectrum, ${ }^{22,23}$ this minimal coupling scheme is favored, because of its technical simplicity, to be chosen as the basis for study of impurity effects in LaOFeAs which would hardly be tractable in more involved frameworks.

Having established the SC-state parameters, an important class of problems can be considered about the effects of disorder, in particular, by impurities, on the system electronic properties, and this issue has also been studied for doped ferropnictides. Like the situation in doped perovskite cuprates, here impurity centers can either result from the dopants, necessary to form the very SC state, or from foreign atoms and other local defects in the crystalline structure. Within the minimal coupling model, the interesting possibility of localized impurity levels appearing within SC gaps in doped $\mathrm{LaOFeAs}$ was indicated, even for the simplest, so-called isotopic (or nonmagnetic) type of impurity perturbation. ${ }^{24,25}$ This finding marks an essential difference from traditional $\mathrm{SC}$ systems with an $s$-wave gap on a single-connected Fermi surface, where such perturbations are known not to produce localized impurity states and thus to have no sizable effect on SC order, according to the Anderson theorem. ${ }^{26}$ In the presence of localized quasiparticle states by isolated impurity centers, the next important issue is the possibility of collective behavior of such states at high enough impurity concentrations. This possibility was studied long ago for electronic quasiparticles in doped semiconducting systems ${ }^{27}$ and also for other types of quasiparticles in pnononic, magnonic, excitonic, etc., spectra under impurities, ${ }^{28}$ establishing conditions for collective (including coherent) behavior of impurity excitations, with striking effects in the observable properties of such systems. As for high- $\mathrm{T}_{c}$-doped cuprates, it is known that their $d$-wave symmetry of SC order permits only impurity resonances in the spectrum of quasiparticles, ${ }^{29,30}$ not their true localization, and hinders notable collective effects on their observable properties. To our knowledge, no consistent study on collective impurity effects is known for doped ferropnictide systems at present, and this defines the main emphasis of the present work. 


\title{
Specifics of impurity effects in ferropnictide superconductors
}

\author{
Y.G. Pogorelov, ${ }^{1}$ M.C. Santos, ${ }^{2}$ V.M. Loktev ${ }^{3}$ \\ ${ }^{1}$ IFIMUP-IN, Departamento de Física, Universidade do Porto, Porto, \\ Portugal, \\ ${ }^{2}$ Departamento de Física, Universidade de Coimbra, R. Larga, Coimbra, \\ 3004-535, Portugal, \\ ${ }^{3}$ Bogolyubov Institute for Theoretical Physics, NAN of Ukraine, 14b \\ Metrologichna str., $03143 \mathrm{Kiev}$, Ukraine
}

Effects of impurities and disorder on quasiparticle spectrum in superconducting iron pnictides are considered.The possibility of occurrence of localized energy levels due to impurities within the superconducting gap and therelated modification of band structure, including the emergence of narrow bands of extended quasiparticle statesnear impurity levels, is analyzed. The evolution of a superconducting state with an impurity concentration istraced and some specific effects of the modified quasiparticle spectrum on the superconducting order parameterand other observable characteristics are discussed.

\section{Introduction}

The recent discovery of superconductivity (SC) with rather high critical temperature in the family of doped ferropnictide compounds $[140,141]$ has motivated a great interest to these materials (see Reviews in Refs. [142] and [143]). Unlike the extensively studied cuprate family, [144] which presents insulating properties in their initial undoped state, the undoped LaOFeAs compound is a semimetal. As established in previous physical and chemical studies (see, e.g., Refs. [145] and [146]), this material has a layered structure, where the SC state is suported by the FeAs layer with a two-dimensional (2D) square lattice of $\mathrm{Fe}$ atoms and with As atoms located out of plane, above or below the centers of square cells (Fig. 4.1). Its electronic structure, relevant for constructing microscopic SC models, have been explored with high-resolution angle-resolved photoemission spectroscopy (ARPES) techniques. [147, 148] Their results indicate the multiple connected structure of Fermi surface, consisting of electron and hole pockets and absence of nodes in both electron and hole gaps, [147] suggesting that these systems display the so-called extended $s$-wave (also called $s_{ \pm}$wave) SC order, changing the order parameter sign between electron and hole segments. [149]

To study band structure, first principles numeric calculations are commonly used, outlining the importance of Fe atomic $d$-orbitals. The calculations show 
that SC in these materials is associated with Fe atoms in the layer plane, represented in Fig. 4.1 by their orbitals and the related hopping amplitudes. The dominance of Fe atomic $3 d$ orbitals in the density of states (DOS) of LaOFeAs compound near its Fermi surface was demonstrated by local density approximation (LDA) calculations. [149, 150, 151, 152, 153, 154] It was then concluded that the multiorbital effects are important for electronic excitation spectrum in the SC state, causing formation of two spectrum gaps: by electron and hole pockets at the Fermi surface. To explain the observed SC properties, it is suggested that these materials may reveal an unconventional pairing mechanism, beyond the common electron-phonon scheme. [155, 156, 157, 158] In general, a total of five atomic orbitals for each iron in the LaOFeAs compound can be involved, however, ways to reduce this basis are sought, in order to simplify analytical and computational work. Some authors $[159,160]$ have suggested that it is sufficient to consider only the $d_{x z}$ and $d_{y z}$ orbitals. Building such minimal coupling model based on two orbitals, one is able to adjust the model parameters (energy hopping and chemical potential) to obtain the Fermi surface with the same topology as in the first-principles calculations of band structure. Even though it fails to reproduce some finer features of the electronic spectrum, $[161,162]$ this minimal coupling scheme is favored, because of its technical simplicity, to be chosen as the basis for study of impurity effects in $\mathrm{LaOFeAs}$ which would hardly be tractable in more involved frameworks.

Having established the SC-state parameters, an important class of problems can be considered about the effects of disorder, in particular, by impurities, on the system electronic properties, and this issue has been also studied for doped ferropnictides. Like the situation in doped perovskite cuprates, here impurity centers can either result from the dopants, necessary to form the very SC state, or from foreign atoms and other local defects in the crystalline structure. Within the minimal coupling model, an interesting possibility for localized impurity levels appearing within SC gaps in doped LaOFeAs was indicated, even for the simplest, so-called isotopic (or nonmagnetic) type of impurity perturbation. [163, 164] This finding marks an essential difference from the traditional $\mathrm{SC}$ systems with $s$-wave gap on a single-connected Fermi surface, were such perturbations are known not to produce localized impurity states and thus to have no sizeable effect on SC order, according to the Anderson theorem. [113] In presence of localized quasiparticle states by isolated impurity centers, the next important issue is the possibility of collective behavior of such states at high enough impurity concentrations. This possibility was studied long ago for electronic quasiparticles in doped semiconducting systems [165] and also for other types of quasiparticles in pnononic, magnonic, excitonic, etc., spectra under impurities, [132] establishing conditions for collective (including 


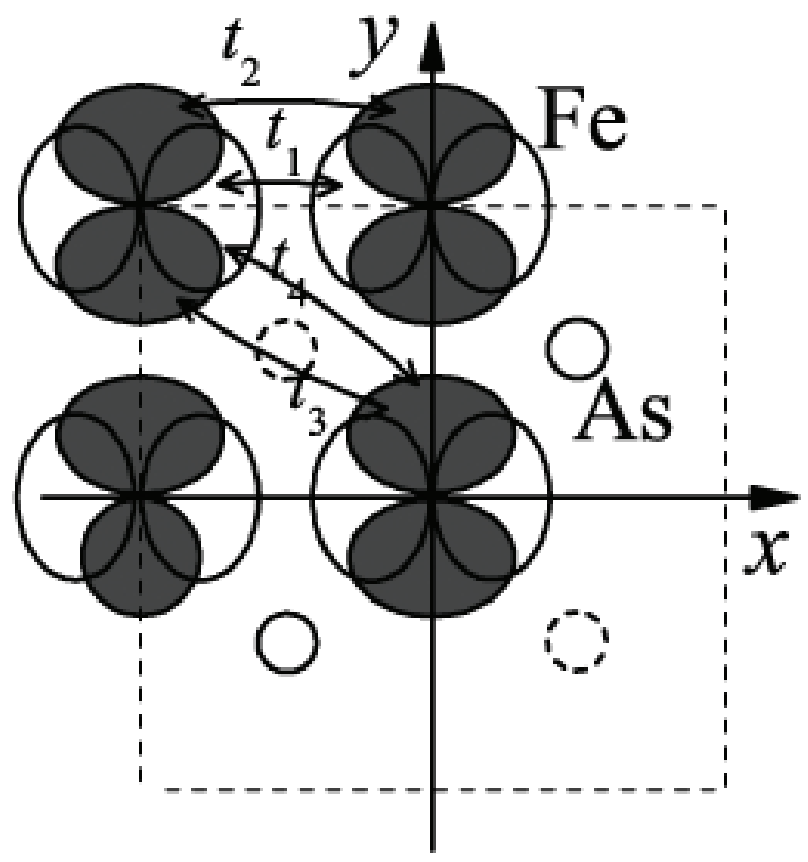

Figure 4.1: Schematics of a FeAs layer in the LaoFeAs compound with $d_{x z}$ (white) and $d_{y z}$ (dark) Fe orbitals and the Fe-Fe hopping parameters in the minimal coupling model. Note that the hoppings between next near neighbors $\left(t_{3,4}\right)$ are mediated by the As orbitals (out of Fe plane).

coherent) behavior of impurity excitations, with striking effects in observable properties of such systems. As for high- $\mathrm{T}_{c}$ doped cuprates, it is known that their $d$-wave symmetry of SC order permits only existence of impurity resonances in the spectrum of quasiparticles, [?, 115] not their true localization, and hinders notable collective effects on their observable properties. To our knowledge, no consistent study on collective impurity effects is know for the doped ferropnictide systems at present, and this defines the main emphasis of the present work.

Namely, we develop an analysis of these systems, using the Green function (GF) techniques, similar to those for doped cuprate SC systems, [22] the minimal coupling model by two orbitals for ferropnictide electronic structure, and the simplest isotopic type for impurity perturbation. The structure of quasiparticle spectrum near in-gap impurity levels at finite impurity concentrations, conditions for emergence of specific branches of collective excitations in this 
region of the spectrum, and expected observable effects of such spectrum restructuring will be discussed.

\section{Model Hamiltonian and Green Functions}

For the minimal coupling model of Fig. 4.1, the hopping Hamiltonian $H_{t}$ is written in the local orbital basis as

$$
\begin{aligned}
H_{t} & =-\sum_{\mathbf{n}, \sigma}\left[t_{1}\left(x_{\mathbf{n}, \sigma}^{\dagger} x_{\mathbf{n}+\boldsymbol{\delta}_{x}, \sigma}+y_{\mathbf{n}, \sigma}^{\dagger} y_{\mathbf{n}+\boldsymbol{\delta}_{y}, \sigma}+h . c .\right)\right. \\
& +t_{2}\left(x_{\mathbf{n}, \sigma}^{\dagger} x_{\mathbf{n}+\boldsymbol{\delta}_{y}, \sigma}+y_{\mathbf{n}, \sigma}^{\dagger} y_{\mathbf{n}+\boldsymbol{\delta}_{x}, \sigma}+h . c .\right) \\
& +t_{3}\left(x_{\mathbf{n}, \sigma}^{\dagger} x_{\mathbf{n}+\boldsymbol{\delta}_{x}+\boldsymbol{\delta}_{y}, \sigma}+x_{\mathbf{n}, \sigma}^{\dagger} x_{\mathbf{n}+\boldsymbol{\delta}_{x}-\boldsymbol{\delta}_{y}, \sigma}\right. \\
& \left.+y_{\mathbf{n}, \sigma}^{\dagger} y_{\mathbf{n}+\boldsymbol{\delta}_{x}+\boldsymbol{\delta}_{y}, \sigma}+y_{\mathbf{n}, \sigma}^{\dagger} y_{\mathbf{n}+\boldsymbol{\delta}_{x}-\boldsymbol{\delta}_{y}, \sigma}+h . c .\right) \\
& +t_{4}\left(x_{\mathbf{n}, \sigma}^{\dagger} y_{\mathbf{n}+\boldsymbol{\delta}_{x}+\boldsymbol{\delta}_{y}, \sigma}+y_{\mathbf{n}, \sigma}^{\dagger} x_{\mathbf{n}+\boldsymbol{\delta}_{x}+\boldsymbol{\delta}_{y}, \sigma}\right. \\
& \left.\left.-x_{\mathbf{n}, \sigma}^{\dagger} y_{\mathbf{n}+\boldsymbol{\delta}_{x}-\boldsymbol{\delta}_{y}, \sigma}-y_{\mathbf{n}, \sigma}^{\dagger} x_{\mathbf{n}+\boldsymbol{\delta}_{x}-\boldsymbol{\delta}_{y}, \sigma}+\text { h.c. }\right)\right] .
\end{aligned}
$$

where $x_{\mathbf{n}, \sigma}$ and $y_{\mathbf{n}, \sigma}$ are the Fermi operators for $d_{x z}$ and $d_{y z}$ Fe orbitals with spin $\sigma$ on $\mathbf{n}$ lattice site and the vectors $\boldsymbol{\delta}_{x, y}$ point to its nearest neighbors in the square lattice. Passing to the operators of orbital plane waves $x_{\mathbf{k}, \sigma}=$ $N^{-1 / 2} \sum_{\mathbf{n}} \mathrm{e}^{i \mathbf{k} \cdot \mathbf{n}} x_{\mathbf{n}, \sigma}$ (with the number $N$ of lattice cells) and analogous $y_{\mathbf{k}, \sigma}$, and defining an "orbital" 2-spinor $\psi^{\dagger}(\mathbf{k}, \sigma)=\left(x_{\mathbf{k}, \sigma}, y_{\mathbf{k}, \sigma}\right)$, one can expand the spinor Hamiltonian in quasimomentum:

$$
H_{t}=\sum_{\mathbf{k}, \sigma} \psi^{\dagger}(\mathbf{k}, \sigma) \hat{h}_{t}(\mathbf{k}) \psi(k, \sigma) .
$$

Here the $2 \times 2$ matrix,

$$
\hat{h}_{t}(\mathbf{k})=\varepsilon_{+, \mathbf{k}} \hat{\sigma}_{0}+\varepsilon_{-, \mathbf{k}} \hat{\sigma}_{3}+\varepsilon_{x y, \mathbf{k}} \hat{\sigma}_{1},
$$

includes the Pauli matrices $\hat{\sigma}_{i}$ and the energy functions

$$
\varepsilon_{ \pm, \mathbf{k}}=\frac{\varepsilon_{x, \mathbf{k}} \pm \varepsilon_{x, \mathbf{k}}}{2},
$$

with 


$$
\begin{aligned}
\varepsilon_{x, \mathbf{k}} & =-2 t_{1} \cos k_{x}-2 t_{2} \cos k_{y}-4 t_{3} \cos k_{x} \cos k_{y} \\
\varepsilon_{y, \mathbf{k}} & =-2 t_{1} \cos k_{y}-2 t_{2} \cos k_{x}-4 t_{3} \cos k_{x} \cos k_{y} \\
\varepsilon_{x y, \mathbf{k}} & =-4 t_{4} \sin k_{x} \sin k_{y} .
\end{aligned}
$$

The optimum fit for the calculated band structure within the minimum coupling model is attained with the following set of hopping parameters (in $\left|t_{1}\right|$ units): $t_{1}=-1.0, t_{2}=1.3, t_{3}=t_{4}=-0.85$, and with the choice of the Fermi energy (chemical potential at zero temperature) $\varepsilon_{\mathrm{F}}=1.45$. [154] The $\hat{h}_{t}$ matrix is diagonalized by the standard unitary transformation:

$$
\hat{U}(\mathbf{k})=\left(\begin{array}{cc}
\cos \theta_{\mathbf{k}} / 2 & -\sin \theta_{\mathbf{k}} / 2 \\
\sin \theta_{\mathbf{k}} / 2 & \cos \theta_{\mathbf{k}} / 2
\end{array}\right)
$$

with $\theta_{\mathbf{k}}=\arctan \left(\varepsilon_{x y, \mathbf{k}} / \varepsilon_{-, \mathbf{k}}\right)$, transforming it from an orbital to a sub-band basis:

$$
\hat{h}_{b}(\mathbf{k})=\hat{U}^{\dagger}(\mathbf{k}) \hat{h}_{t}(\mathbf{k}) \hat{U}(\mathbf{k})=\left(\begin{array}{cc}
\varepsilon_{e, \mathbf{k}} & 0 \\
0 & \varepsilon_{h, \mathbf{k}}
\end{array}\right)
$$

The energy eigenvalues in Eq. (4.4):

$$
\varepsilon_{h, e}(\mathbf{k})=\varepsilon_{+, \mathbf{k}} \pm \sqrt{\varepsilon_{x y, \mathbf{k}}^{2}+\varepsilon_{-, \mathbf{k}}^{2}},
$$

correspond to the two sub-bands in the normal-state spectrum that define electron and hole pockets of the Fermi surface, respectively. There are two segments of each type, defined by the equations $\varepsilon_{e, h}(\mathbf{k})=\mu$, as shown in Fig. 4.2. We note that both functions $\cos \theta_{\mathbf{k}}$ and $\sin \theta_{\mathbf{k}}$ change their sign around these segments, corresponding to their "azimuthal dependencies" around characteristic points of the Brillouin zone (Fig. 4.2), so that integrals of these functions with some azimuthal-independent factors over the relevant vicinity of Fermi surface practically vanish and are neglected beside such integrals of fully azimuthal-independent functions in the analysis below.

The adequate basis for constructing the SC state is generated by the operators of electron and hole sub-bands:

$$
\begin{aligned}
\alpha_{\mathbf{k}, \sigma} & =x_{\mathbf{k}, \sigma} \cos \theta_{\mathbf{k}} / 2-y_{\mathbf{k}, \sigma} \sin \theta_{\mathbf{k}} / 2, \\
\beta_{\mathbf{k}, \sigma} & =y_{\mathbf{k}, \sigma} \cos \theta_{\mathbf{k}} / 2+x_{\mathbf{k}, \sigma} \sin \theta_{\mathbf{k}} / 2,
\end{aligned}
$$




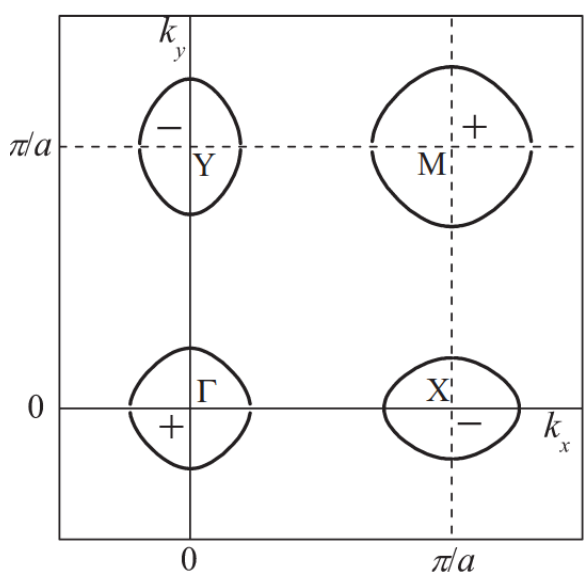

Figure 4.2: Electron $(-)$ and hole $(+)$ segments of the Fermi surface in the normal state of model system with electronic spectrum from Eq. (4.5). The center of first Brillouin zone is displaced by $(\pi / 2 a, \pi / 2 a)$ to fully include all the segments around four characteristic points $\Gamma, \mathrm{X}, \mathrm{M}$, and $\mathrm{Y}$ in this zone.

giving rise to the "multiband-Nambu" 4-spinors $\Psi_{\mathbf{k}}^{\dagger}=\left(\alpha_{\mathbf{k}, \uparrow}^{\dagger}, \alpha_{-\mathbf{k}, \downarrow}, \beta_{\mathbf{k}, \uparrow}^{\dagger}, \beta_{-\mathbf{k}, \downarrow}\right)$ and to a $4 \times 4$ extension of the Hamiltonian, Eq. (4.2), in the form

$$
H_{s}=\sum_{\mathbf{k}, \sigma} \Psi_{\mathbf{k}}^{\dagger} \hat{h}_{s}(\mathbf{k}) \Psi_{\mathbf{k}}
$$

where the $4 \times 4$ matrix

$$
\hat{h}_{s}(\mathbf{k})=\hat{h}_{b}(\mathbf{k}) \otimes \hat{\tau}_{3}+\Delta_{\mathbf{k}} \hat{\sigma}_{0} \otimes \hat{\tau}_{1}
$$

includes the Pauli matrices $\hat{\tau}_{i}$ acting on the Nambu (particle-antiparticle) indices in $\Psi$-spinors and $\hat{h}_{b}(\mathbf{k})$ is defined by Eq. (4.5). The simplified form for the extended $s$-wave $\mathrm{SC}$ order is realized with the definition of the gap function by constant values, $\Delta_{\mathrm{k}}=\Delta$ on the electron segments and $\Delta_{\mathrm{k}}=-\Delta$ on the hole segments.

The electronic dynamics of this system is determined by the (Fourier transformed) GF $4 \times 4$ matrices $[132,22,166]$

$$
\hat{G}_{\mathbf{k}, \mathbf{k}^{\prime}}=\left\langle\left\langle\Psi_{\mathbf{k}} \mid \Psi_{\mathbf{k}}^{\dagger}\right\rangle\right\rangle=i \int_{-\infty}^{0} d t \mathrm{e}^{i \varepsilon t / \hbar}\left\langle\left\{\Psi_{\mathbf{k}}(t), \Psi_{\mathbf{k}^{\prime}}^{\dagger}(0)\right\}\right\rangle,
$$


whose energy argument $\varepsilon$ is understood as $\varepsilon-i 0$ and $\langle\{A(t), B(0)\}\rangle$ is the quantum statistical average with Hamiltonian $H$ of the anticommutator of Heisenberg operators. From the equation of motion,

$$
\varepsilon \hat{G}_{\mathbf{k}, \mathbf{k}^{\prime}}=\hbar \delta_{\mathbf{k}, \mathbf{k}^{\prime}} \hat{\sigma}_{0} \otimes \tau_{0}+\left\langle\left\langle\left[\Psi_{\mathbf{k}}, H\right] \mid \Psi_{\mathbf{k}^{\prime}}^{\dagger}\right\rangle\right\rangle,
$$

the explicit GF for the unperturbed $\mathrm{SC}$ system with the Hamiltonian $H_{s}$, Eq. (4.7), is diagonal in quasimomentum, $\hat{G}_{\mathbf{k}, \mathbf{k}^{\prime}}=\delta_{\mathbf{k}, \mathbf{k}^{\prime}} \hat{G}_{\mathbf{k}}^{0}$ and

$$
\begin{aligned}
\hat{G}_{\mathbf{k}}^{0} & =\frac{\varepsilon \hat{\tau}_{0}+\varepsilon_{e}(\mathbf{k}) \hat{\tau}_{3}+\Delta \hat{\tau}_{1}}{2 D_{e, \mathbf{k}}} \otimes \hat{\sigma}_{+} \\
& +\frac{\varepsilon \hat{\tau}_{0}+\varepsilon_{h}(\mathbf{k}) \hat{\tau}_{3}-\Delta \hat{\tau}_{1}}{2 D_{h, \mathbf{k}}} \otimes \hat{\sigma}_{-},
\end{aligned}
$$

where $\hat{\sigma}_{ \pm}=\left(\hat{\sigma}_{0} \pm \hat{\sigma}_{3}\right) / 2$ and the secular denominators $D_{i, \mathbf{k}}=\varepsilon^{2}-\varepsilon_{i}^{2}(\mathbf{k})-$ $\Delta^{2}$ for $i=e, h$. In what follows, we use the energy reference to the Fermi level $\varepsilon_{\mathrm{F}}$ and approximate the segments of Fermi surface by some circles of radius $k_{i}$ around the characteristic points $\mathbf{K}_{i}$ in the Brillouin zone, so that the dispersion laws $\varepsilon_{j}(\mathbf{k})=\varepsilon_{\mathrm{F}}+\xi_{j, \mathbf{k}}$ permit linearization of the quasiparticle dispersion close to the Fermi level as $\xi_{j, \mathbf{k}} \approx \hbar v_{j}\left(\left|\mathbf{k}-\mathbf{K}_{j}\right|-k_{i}\right)$. Generally, the Fermi wave numbers $k_{j}$ and related Fermi velocities $v_{j}$ for $j=e$ and $h$ can differ somewhat at a given choice of hopping parameters and chemical potential, but for simplicity, we neglect this difference and consider their single values $k_{j}=k_{\mathrm{F}}$ and $v_{j}=v_{\mathrm{F}}$.

\section{Impurity Perturbation and Self-Energy}

We pass to the impurity problem, where the above Hamiltonian is added by the local perturbation terms due to nonmagnetic impurities [164] on random sites $\mathbf{p}$ in Fe square lattice with an on-site energy shift $V$ :

$$
H_{i m p}=V \sum_{\mathbf{p}, \sigma}\left(x_{\mathbf{p}, \sigma}^{\dagger} x_{\mathbf{p}, \sigma}+y_{\mathbf{p}, \sigma}^{\dagger} y_{\mathbf{p}, \sigma}\right) .
$$

Without loss of generality, the parameter $V$ can be taken positive, and for GF calculations, this perturbation is suitably expressed in the multiband-Nambu basis, 


$$
H_{i m p}=\frac{1}{N} \sum_{\mathbf{p}, \mathbf{k}, \mathbf{k}^{\prime}} \mathrm{e}^{i\left(\mathbf{k}^{\prime}-\mathbf{k}\right) \cdot \mathbf{p}} \Psi_{\mathbf{k}}^{\dagger} \hat{V}_{\mathbf{k}, \mathbf{k}^{\prime}} \Psi_{\mathbf{k}^{\prime}}
$$

through the $4 \times 4$ scattering matrix $\hat{V}_{\mathbf{k}, \mathbf{k}^{\prime}}=V \hat{U}_{\mathbf{k}}^{\dagger} \hat{U}_{\mathbf{k}^{\prime}} \otimes \tau_{3}$. As follows from the above expression for $\hat{U}_{\mathbf{k}}$, this matrix involves either "intraband" or "interband" elements.[166] The latter type of scattering could lead to a possible transformation from the $s_{ \pm}$to a competing $s_{++}$SC order (with the same sign of order parameter on both Fermi pockets) under the impurity effect.[167] However, as shown below, such a possibility is effectively eliminated for the chosen local perturbation type, due to the specific quasimomentum $\mathbf{k}$ dependence of the scattering elements, unlike their constancy, postulated in Ref. [167].

Within the approach of Refs. [132] and [22], the solution for Eq. (4.9) with the perturbed Hamiltonian $H_{s}+H_{i}$ can be obtained in different forms, suitable for different types of states, band-like (extended) or localized. All these forms result from the basic equation of motion,

$$
\hat{G}_{\mathbf{k}, \mathbf{k}^{\prime}}=\delta_{\mathbf{k}, \mathbf{k}^{\prime}} \hat{G}_{\mathbf{k}}^{0}+\frac{1}{N} \sum_{\mathbf{p}, \mathbf{k}^{\prime \prime}} \mathrm{e}^{i\left(\mathbf{k}^{\prime \prime}-\mathbf{k}\right) \cdot \mathbf{p}} \hat{G}_{\mathbf{k}}^{0} \hat{V}_{\mathbf{k}, \mathbf{k}^{\prime \prime}} \hat{G}_{\mathbf{k}^{\prime \prime}, \mathbf{k}^{\prime}}
$$

by specific routines of iterating this equation for the "scattered" GFs $\hat{G}_{\mathbf{k}^{\prime \prime}, \mathbf{k}^{\prime}}$.

Thus, the algorithm, where the next iteration step never applies to the scattered GFs already present after previous steps, e.g. that with $\mathbf{k}^{\prime \prime}=\mathbf{k}$ in Eq. (4.14), leads to the so-called fully renormalized form, suitable for band-like states,

$$
\hat{G}_{\mathbf{k}}=\left[\left(\hat{G}_{\mathbf{k}}^{0}\right)^{-1}-\hat{\Sigma}_{\mathbf{k}}\right]^{-1}
$$

where the self-energy matrix $\hat{\Sigma}_{\mathbf{k}}$ is expressed by the related group expansion $(\mathrm{GE})$ :

$$
\hat{\Sigma}_{\mathbf{k}}=c \hat{T}_{\mathbf{k}}\left(1+c \hat{B}_{\mathbf{k}}+\ldots\right)
$$

Here $c=\sum_{\mathbf{p}} N^{-1}$ is the impurity concentration (per Fe site) and the $T$ matrix results from all the multiple scatterings by a single impurity: 


$$
\begin{aligned}
\hat{T}_{\mathbf{k}} & =\hat{V}_{\mathbf{k}, \mathbf{k}}+\frac{1}{N} \sum_{\mathbf{k}^{\prime} \neq \mathbf{k}} \hat{V}_{\mathbf{k}, \mathbf{k}^{\prime}} \hat{G}_{\mathbf{k}^{\prime}}^{0} \hat{V}_{\mathbf{k}^{\prime}, \mathbf{k}} \\
& +\frac{1}{N^{2}} \sum_{\mathbf{k} \neq \mathbf{k}, \mathbf{k}^{\prime \prime} \neq \mathbf{k}, \mathbf{k}^{\prime}} \hat{V}_{\mathbf{k}, \mathbf{k}^{\prime}} \hat{G}_{\mathbf{k}^{\prime}}^{0} \hat{V}_{\mathbf{k}^{\prime}, \mathbf{k}^{\prime \prime}} \hat{G}_{\mathbf{k}^{\prime \prime}}^{0} \hat{V}_{\mathbf{k}^{\prime \prime}, \mathbf{k}}+\ldots
\end{aligned}
$$

The next term to the unity in the brackets in Eq. (4.16),

$$
\hat{B}_{\mathbf{k}}=\sum_{\mathbf{n}}\left(\hat{A}_{\mathbf{n}} \mathrm{e}^{-i \mathbf{k} \cdot \mathbf{n}}+\hat{A}_{\mathbf{n}} \hat{A}_{-\mathbf{n}}\right)\left(1-\hat{A}_{\mathbf{n}} \hat{A}_{-\mathbf{n}}\right)^{-1}
$$

describes the effects of indirect interactions in pairs of impurities, separated by vector $\mathbf{n}$, in terms of interaction matrices $\hat{A}_{\mathbf{n}}=\hat{T}_{\mathbf{k}} \sum_{\mathbf{k}^{\prime} \neq \mathbf{k}} \mathrm{e}^{i \mathbf{k}^{\prime} \cdot \mathbf{n}} \hat{G}_{\mathbf{k}^{\prime}}$. Besides this restriction on summation, multiple sums in the products like $\hat{A}_{\mathbf{n}} \hat{A}_{-\mathbf{n}}$ never contain coincident quasimomenta. Eq. (4.18) presents the first non-trivial GE term and the rest of its terms omitted in Eq. (4.14) correspond to the contributions from groups of three and more impurities. [132]

An alternative iteration routine for Eq. (4.14) applies it to all the scattered GFs; this results in the so-called nonrenormalized form, suitable for localized states:

$$
\hat{G}_{\mathbf{k}}=\hat{G}_{\mathbf{k}}^{0}+\hat{G}_{\mathbf{k}}^{0} \hat{\Sigma}_{\mathbf{k}}^{0} \hat{G}_{\mathbf{k}}^{0}
$$

Here the non-renormalized self-energy GE, $\hat{\Sigma}_{\mathbf{k}}^{0}=c \hat{T}\left(1+c \hat{B}_{\mathbf{k}}^{0}+\ldots\right)$, differs from the above renormalized one by absence of restrictions in quasimomentum sums for interaction matrices $\hat{A}_{\mathbf{n}}^{0}=\hat{T}_{\mathbf{k}} \sum_{\mathbf{k}^{\prime}} \mathrm{e}^{i \mathbf{k}^{\prime} \cdot \mathbf{n}} \hat{G}_{\mathbf{k}^{\prime}}^{0}$ and their products.

In the first step, we shall restrict GE to the common $T$-matrix level, providing the conditions for localized quasiparticle states with in-gap energy levels to appear at single impurities, [160] and study certain (narrow) energy bands of specific collective states that can be formed near these levels at finite impurity concentrations. At the next step, the criteria for such collective states to really exist in the disordered SC system will follow from the analysis of nontrivial GE terms. We note that presence of renormalized GFs $\hat{G}_{\mathbf{k}^{\prime}}$ in the above interaction matrices is just necessary for adequate treatment of interaction effects over the in-gap bands. 


\section{T-Matrix and Quasiparticle States}

The T-matrix, Eq. (4.16), is readily simplified taking into account that $\hat{V}_{\mathbf{k}, \mathbf{k}}=$ $V \hat{\sigma}_{0} \otimes \hat{\tau}_{3}$ and introducing the integrated GF matrix:

$$
\hat{G}_{0}=\frac{1}{N} \sum_{\mathbf{k}} \hat{U}_{\mathbf{k}} \hat{G}_{\mathbf{k}}^{0} U_{\mathbf{k}}^{\dagger}=\varepsilon\left[g_{e}(\varepsilon) \hat{\sigma}_{+}+g_{h}(\varepsilon) \hat{\sigma}_{-}\right] \otimes \hat{\tau}_{0} .
$$

This diagonal form (that is, restricted only to the "intraband" matrix elements) follows directly from the aforementioned cancellation of the integrals with $\cos \theta_{\mathbf{k}}$ and $\sin \theta_{\mathbf{k}}$ that appear in the interband matrix elements of $\hat{U}_{\mathbf{k}} \hat{G}_{\mathbf{k}}^{0} U_{\mathbf{k}}^{\dagger}$. Therefore, we do not consider below that $\mathrm{SC}$ order can change its type under impurity effects.

Respectively, the functions $g_{j}(\varepsilon)=N^{-1} \sum_{\mathbf{k}} D_{j, \mathbf{k}}^{-1}$ for $j=e, h$ are approximated near the Fermi level, $\left|\varepsilon-\varepsilon_{\mathrm{F}}\right| \lesssim \Delta$, as,

$$
g_{j}(\varepsilon) \approx-\frac{\pi \rho_{j}}{\sqrt{\Delta^{2}-\varepsilon^{2}}} .
$$

Here $\rho_{j}=m_{j} a^{2} /\left(2 \pi \hbar^{2}\right)$ are the Fermi densities of states for respective subbands (in parabolic approximation for their dispersion laws), and by the assumed identity of all the segments of Fermi surface, they can be also considered identical, $\rho_{j}=\rho_{\mathrm{F}}$. Omitted terms in Eq. (4.16) are of higher orders in the small parameter $|\varepsilon| / \varepsilon_{\mathrm{F}} \ll 1$.

Then the momentum independent $T$ matrix is explicitly written as

$$
\hat{T}=\gamma^{2} \frac{\varepsilon-\varepsilon_{0} \hat{\tau}_{3}}{\varepsilon^{2}-\varepsilon_{0}^{2}},
$$

where $\varepsilon_{0}=\Delta / \sqrt{1+v^{2}}$ defines the in-gap impurity level [160] through the dimensionless impurity perturbation parameter $v=\pi \rho_{\mathrm{F}} V$, and $\gamma^{2}=v^{2} V \varepsilon_{0}^{2} / \Delta$ is the effective constant of coupling between localized and band quasiparticles. Evidently, Eq. (4.21) is only valid in a narrow enough vicinity of $\varepsilon_{0}$.

At finite $c$, using this $T$ matrix in Eq. (4.14), we obtain, from the condition $\operatorname{det} \hat{G}_{\mathbf{k}}^{-1}=0$, [166] the formal dispersion equation expressed through dispersion of normal quasiparticles $\xi_{\mathbf{k}}=\varepsilon_{\mathbf{k}}-\varepsilon_{\mathrm{F}}$ (but neglecting the energy level width due to the effects of indirect interaction between impurities by higher GE terms): 


$$
D_{\mathbf{k}}(\varepsilon)=\varepsilon^{2}-\xi_{\mathbf{k}}^{2}-\Delta^{2}-\frac{2 c \gamma^{2}\left(\varepsilon^{2}-\varepsilon_{0} \xi_{\mathbf{k}}\right)}{\varepsilon^{2}-\varepsilon_{0}^{2}}=0 .
$$

Its solutions shown in Fig. 4.3 as a function of the quasimomentum argument $\xi=\xi_{\mathbf{k}}$ display a peculiar multiband structure. First, it includes four modified bands $\pm \varepsilon_{b}( \pm \xi)$, slightly shifted with respect to the unperturbed SC quasiparticle bands $\pm \sqrt{\Delta^{2}+\xi^{2}}$, accordingly to the basic function:

$$
\varepsilon_{b}(\xi) \approx \sqrt{\Delta^{2}+\xi^{2}}+c \gamma^{2} \frac{\Delta^{2}+\xi^{2}-\varepsilon_{0} \xi}{\sqrt{\Delta^{2}+\xi^{2}}\left(\xi^{2}+\xi_{0}^{2}\right)},
$$

with $\xi_{0}^{2}=\Delta^{2}-\varepsilon_{0}^{2}$. It should be noted that these sub-bands for opposite signs of their argument $\xi$ in fact refer to excitations around different segments (by electron and holes) of the Fermi surface, but for clarity presented in Fig. 4.3 in the same $\xi$-reference. Besides these $\varepsilon_{b}$ bands, four (narrow) in-gap bands $\pm \varepsilon_{i}( \pm \xi)$ also appear, generated close to $\pm \varepsilon_{0}$ by finite concentrations of impurities, according to

$$
\varepsilon_{i}(\xi) \approx \varepsilon_{0}+c \gamma^{2} \frac{\xi-\varepsilon_{0}}{\xi^{2}+\xi_{0}^{2}}
$$

As follows from Eq. (4.21), the $\varepsilon_{j}(\xi)$ band is located between its extrema $\varepsilon_{\max }=\varepsilon_{0}+c \gamma^{2} \varepsilon_{0} /\left(\Delta+\varepsilon_{0}\right)$ at $\xi_{+}=\varepsilon_{0}+\Delta$ and $\varepsilon_{\min }=\varepsilon_{0}-c \gamma^{2} \varepsilon_{0} /\left(\Delta-\varepsilon_{0}\right)$ at $-\xi_{-}=\varepsilon_{0}-\Delta$. The energy and momentum shifts of the extremal points by Eqs. (4.20) and (4.21) and Fig. 4.3 are specific for the impurity effect on the multiband initial spectrum and they contrast with a simpler situation for an impurity level near the edge of a single-quasiparticle band. [132]

All these spectrum bands would contribute to the overall DOS by related quasiparticles: $\rho(\varepsilon)=(4 \pi N)^{-1} \operatorname{Im} \operatorname{Tr} \sum_{\mathbf{k}} \hat{G}_{\mathbf{k}}$. The more common contributions here come from the $\varepsilon_{b}$ bands and they can be expressed through the Bardeen-Cooper-Schrieffer (BCS) DOS in pure crystal, [168] $\rho_{\mathrm{BCS}}(\varepsilon, \Delta)=$ $\rho_{\mathrm{F}} \varepsilon / \sqrt{\varepsilon^{2}-\Delta^{2}}$, as follows:

$$
\rho_{b}(\varepsilon) \approx\left(1-\frac{c \gamma^{2}}{\varepsilon^{2}-\varepsilon_{0}^{2}}\right) \rho_{\mathrm{BCS}}\left(\varepsilon, \Delta_{c}\right),
$$

at $\varepsilon^{2} \geq \Delta_{c}^{2}=\Delta^{2}+2 c \gamma^{2} \varepsilon_{0}^{2} /\left(\Delta^{2}-\varepsilon_{0}^{2}\right)$. The first factor in the left-hand side of Eq. (4.25) describes a certain reduction of the BCS DOS, especially when the energy argument is close to the gap limits, and the shift of its gap argument 


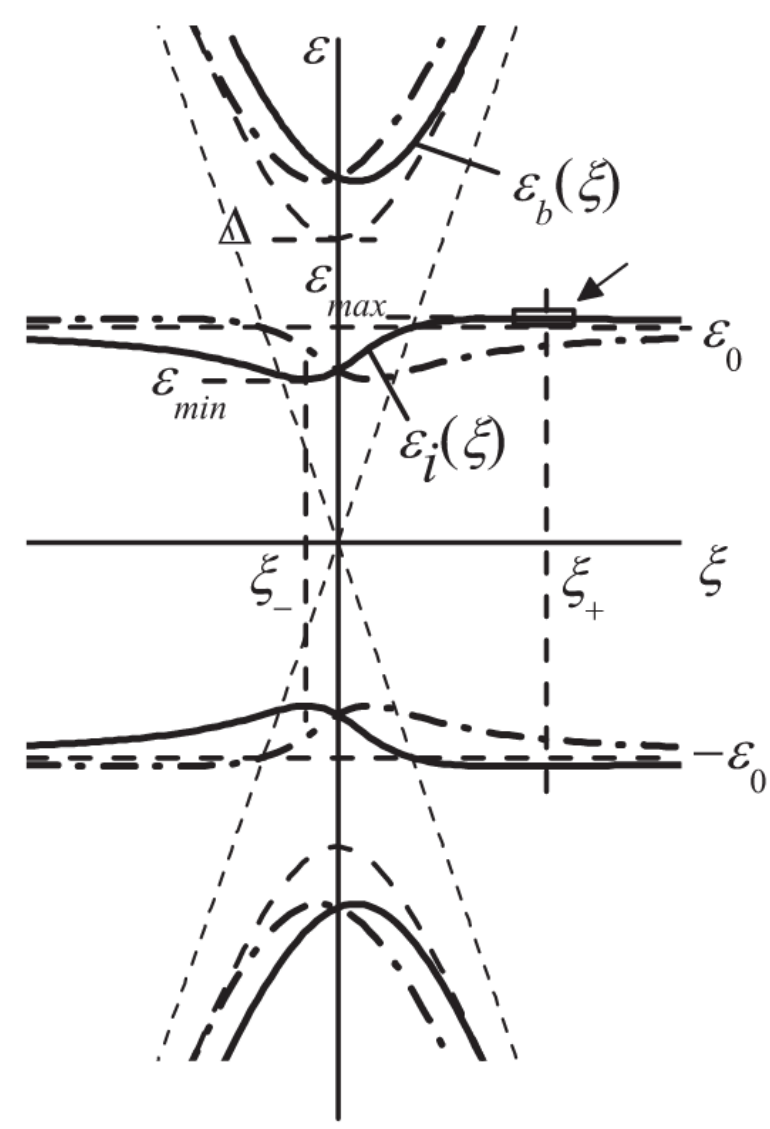

Figure 4.3: Dispersion laws for band-like quasiparticles in the $T$-matrix approximation, neglecting their finite lifetime, at a specific choice of impurity parameters $v=1, c=0.1 \Delta^{2} / \gamma^{2}$. The argument $\xi$ composes all specific $\xi_{j}=\hbar v_{\mathrm{F}}\left(\left|\mathbf{k}-\mathbf{K}_{j}\right|-k_{\mathrm{F}}\right)$ for quasimomentum near each $j$ th characteristic point in the Brillouin zone [see the text after Eq. 4.23)], so that the solid lines presennt the bands near electron-like segments of the Fermi surface, and dash-dotted lines those near hole-like segments. Nonperturbed SC quasiparticle bands and single-impurity localized levels are shown with dashed lines. The narrow rectangle around the top of $\varepsilon_{i}$ band (shown by the arrow) delimits the region in Fig. 4.5. 


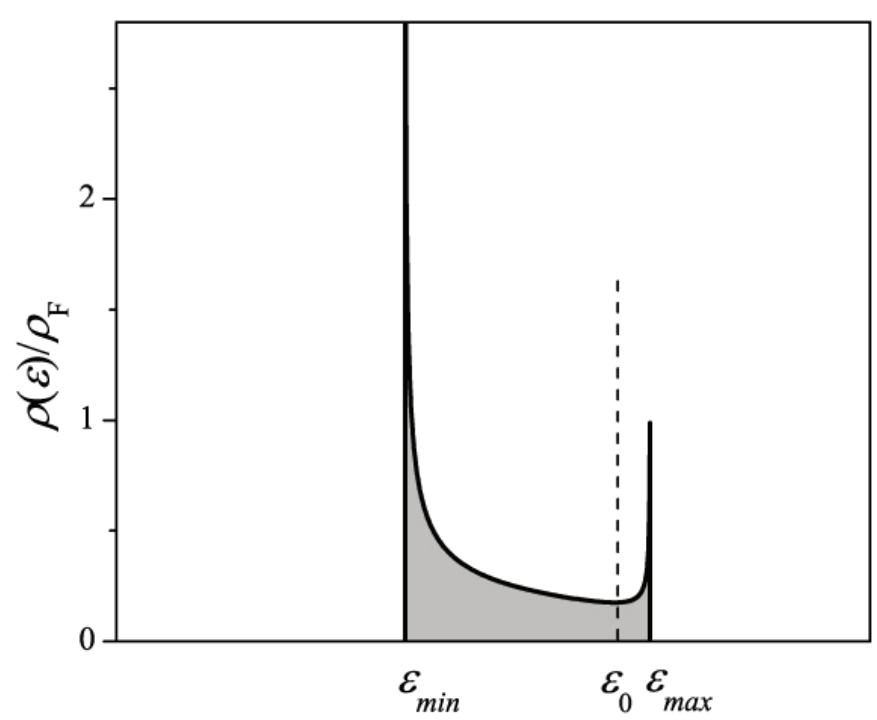

Figure 4.4: Density of states in the narrow in-gap band near the impurity level $\varepsilon_{0}$ (dashed line) for the case in Fig. 4.3 .

is due to the quantum-mechanical repulsion between the band and impurity levels.

More peculiar is the contribution to DOS from the $\varepsilon_{i}$ bands, written as

$$
\rho_{i}(\varepsilon) \approx \frac{\rho_{\mathrm{F}}}{v} \frac{\varepsilon^{2}-\varepsilon_{0}^{2}-c \gamma^{2}}{\sqrt{\left(\varepsilon_{\max }^{2}-\varepsilon^{2}\right)\left(\varepsilon^{2}-\varepsilon_{\min }^{2}\right)}}
$$

at $\varepsilon_{\min }^{2} \leq \varepsilon^{2} \leq \varepsilon_{\text {max }}^{2}$, and presented in Fig. 4.4.

The effects of both $\varepsilon_{b}$ band shifts and of $\varepsilon_{i}$ band formation can have important repercussions for the physical behavior of a disordered SC system and they are considered below. But before this, we need to analyze the criteria for the quasiparticles considered actually to exist, especially in close to the limits of corresponding bands. 


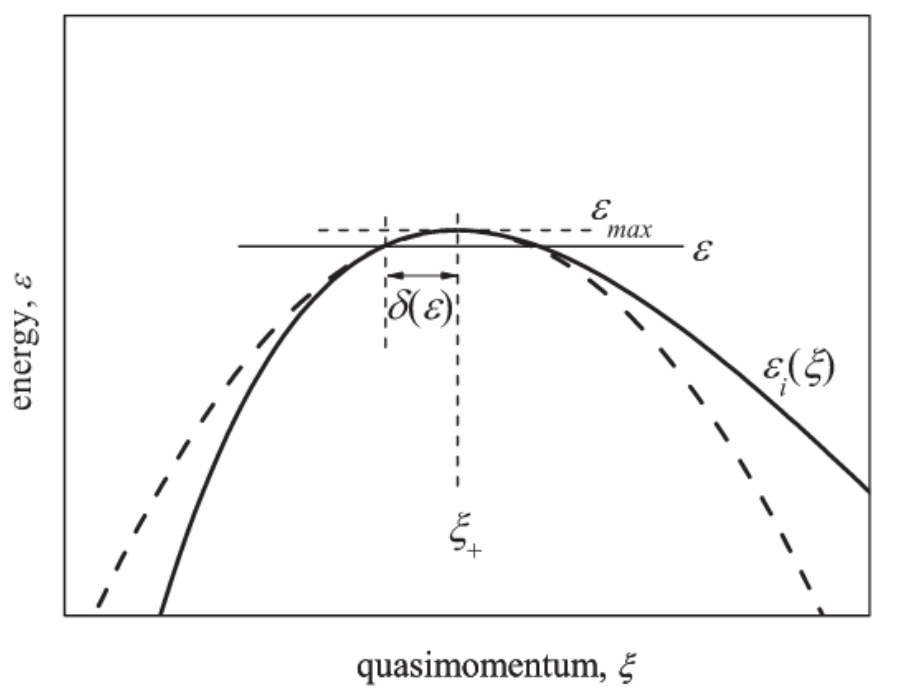

Figure 4.5: Parabolic approximation (dashed line) for the dispersion law near the top of impurity band (solid line), within the region indicated by the small rectangle in Fig. 4.3.

\section{Group Expansion and Coherence Criteria}

Let us now study the crossover from band to localized states near the limits of $\varepsilon_{i}$ bands, say for definiteness, its upper limit $\varepsilon_{\text {max }}$. Supposing the actual energy $\varepsilon<\varepsilon_{\max }$ to be within the range of band states, we use the fully renormalized self-energy matrix, Eq. (4.16), up to the GE pair term, $c^{2} \hat{T} \hat{B}_{\mathbf{k}}$, which will add a certain finite imaginary part $\Gamma_{i}(\xi)$ to the dispersion law $\varepsilon=\varepsilon_{i}(\xi)$, Eq. (4.23). Then the known Ioffe-Regel-Mott criterion [53, 169] for the state at this energy to be really band-like (also called extended) is written as

$$
\varepsilon_{\max }-\varepsilon \gg \Gamma_{i}(\varepsilon)
$$

To simplify calculation of the scalar function $\Gamma_{i}(\varepsilon)$, we fix the energy argument in the numerators of $T$ matrix and interaction matrices at $\varepsilon=\varepsilon_{0}$, obtaining their forms

$$
\hat{T}(\varepsilon) \approx \frac{\gamma^{2} \varepsilon_{0}}{\varepsilon^{2}-\varepsilon_{0}^{2}} \hat{m}_{+}, \quad \hat{A}_{\mathbf{n}}(\varepsilon) \approx \hat{T}(\varepsilon) \frac{\varepsilon_{0}}{N} \sum_{\mathbf{k}} \frac{\mathrm{e}^{i \mathbf{k} \cdot \mathbf{n}}}{D_{\mathbf{k}}(\varepsilon)}
$$


both proportional to the matrix $\hat{m}_{+}=\hat{\sigma}_{0} \otimes\left(\hat{\tau}_{0}+\hat{\tau}_{3}\right)$ with important multiplicative property: $\hat{m}_{+}^{2}=2 \hat{m}_{+}$. The $\mathbf{k}$ summation (integration) in Eq. (4.28) is suitably done in polar coordinates over the circular segments of the Fermi surface. Here the azimuthal integration only refers to the phase of numerator, resulting in a zeroth-order Bessel function: $\int_{0}^{2 \pi} \mathrm{e}^{i x \cos \theta} d \theta=$ $2 \pi J_{0}(x)$. Since $x=n\left(k_{\mathrm{F}}+\xi / \hbar v_{\mathrm{F}}\right)$ is typically big, $x \gg 1$, the asymptotical formula applies: $J_{0}(x) \approx \sqrt{2 /(\pi x)} \cos (x-\pi / 4)$. Then, for radial integration in $\xi$ around the extremum point $\xi_{+}$, it is convenient to decompose this function in the fast and slow oscillating factors, $J_{0}(x) \approx \sqrt{2 /\left(\pi k_{+} n\right)} \cos \left(k_{+} n-\right.$ $\pi / 4) \cos \left[\left(\xi-\xi_{+}\right) n / \hbar v_{\mathrm{F}}\right]$, with the fast wave number $k_{+}=k_{\mathrm{F}}+\xi_{+} / \hbar v_{\mathrm{F}} \approx$ $k_{\mathrm{F}}$, and to write the denominator in the parabolic approximation: $D_{\xi}(\varepsilon) \approx$ $\left(\xi-\xi_{+}\right)^{2}-\delta^{2}(\varepsilon)$, with $\delta^{2}(\varepsilon)=4 \Delta\left(\Delta+\varepsilon_{0}\right)^{2}\left(\varepsilon_{\max }-\varepsilon\right) /\left(2 c \gamma^{2}\right)$ (see Fig. 4.5). Thus, the interaction matrix $\hat{A}_{\mathbf{n}}(\varepsilon)=A_{n}(\varepsilon) \hat{m}_{+}$depends only on the distance $n$ between impurities, and for $\varepsilon$ close to $\varepsilon_{\max }$, this dependence can be expressed as

$$
A_{r}(\varepsilon) \approx \sqrt{\frac{r_{\varepsilon}}{r}} \sin k_{\varepsilon} r \cos k_{\mathrm{F}} r
$$

where the length scales both for the monotonous decay,

$$
r_{\varepsilon}=\frac{2 \pi}{k_{\mathrm{F}}}\left[\frac{\varepsilon_{0} \rho_{\mathrm{F}}\left(\Delta+\varepsilon_{0}\right)}{c \delta(\varepsilon)}\right]^{2}
$$

and for the sine factor: $k_{\varepsilon}^{-1}=\hbar v_{\mathrm{F}} / \delta(\varepsilon)$, are much longer than $k_{\mathrm{F}}^{-1}$ for the fast cosine. The latter fast oscillation is specific for the interactions mediated by Fermi quasiparticles (like the known RKKY mechanism), unlike the monotonous or slowly oscillating interactions between impurities in semiconductors or in bosonic systems. [132] Now the calculation of $\Gamma_{i}(\varepsilon)=c^{2} T(\varepsilon) \operatorname{Im} B(\varepsilon)$ mainly concerns the dominant scalar part of the GE pair term:

$$
B(\varepsilon) \approx \frac{2 \pi}{a^{2}} \int_{a}^{r_{\varepsilon}} \frac{r d r}{1-4 A_{r}^{2}(\varepsilon)}
$$

[since the k-dependent term in Eq. (4.18) turns out to be negligible beside this].

The upper integration limit in Eq. (4.30) corresponds to the condition that its integrand only has poles for $r<r_{\varepsilon}$. In conformity with the slow and fast modes in the function, Eq. (4.29) (see Fig. 4.6), the integration is naturally 


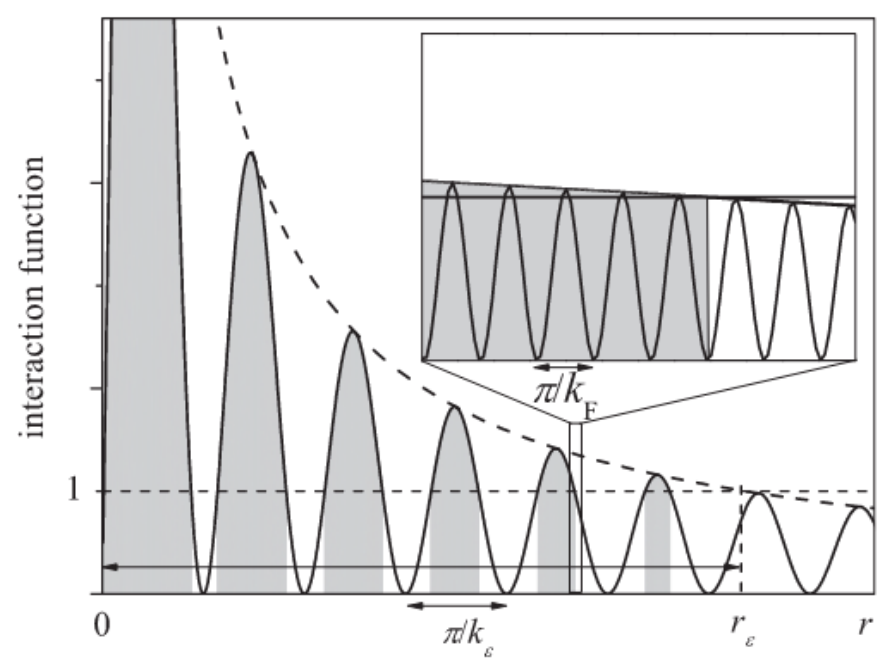

Figure 4.6: Interaction function $A_{r}^{2}(\varepsilon)$ obtained with Eq. (4.29) at the choice of parameters $\varepsilon_{\max }-\varepsilon=0.1$ and $\Delta / \varepsilon_{\mathrm{F}}=5 \cdot 10^{-2}$ displays slow sine oscillations (solid line) and the monotonous envelope function (dashed line). Shaded intervals are those contributing to $\operatorname{Im} B$, according to the condition $\left(r_{e} / r\right) \sin ^{2} k_{\varepsilon} r>1$. Inset: Expansion of the rectangle in the figure also shows fast oscillations by the cosine. 
divided in two stages. At the first stage, integration over each $m$ th period of fast cosine, around $r_{m}=2 \pi m / k_{\mathrm{F}}$, is done by setting constant the slow factors, $r \approx r_{m}$ and $\sin k_{\varepsilon} r \approx \sin k_{\varepsilon} r_{m}$, and using the explicit formula

$$
\operatorname{Im} \int_{-\pi}^{\pi} \frac{d x}{1-4 A^{2} \cos ^{2} x}=\operatorname{Im} \frac{\pi}{\sqrt{1-A^{2}}} .
$$

At the second stage, the summation of these results in $m$ is approximated by the integration in the slow variable

$$
\begin{aligned}
\frac{\pi}{k_{\mathrm{F}}} \operatorname{Im} & \sum_{m} \frac{r_{m}^{3 / 2}}{\sqrt{r_{m}-r_{\varepsilon} \sin ^{2} k_{\varepsilon} r_{m}}} \\
& \approx \operatorname{Im} \int_{a}^{r_{\varepsilon}} \frac{r^{3 / 2} d r}{\sqrt{r-r_{\varepsilon} \sin ^{2} k_{\varepsilon} r}} .
\end{aligned}
$$

The numerical calculation of the latter integral results in

$$
\operatorname{Im} B=\frac{r_{\varepsilon}^{2}}{a^{2}} f\left(k_{\varepsilon} r_{\varepsilon}\right) .
$$

where the function $f(z)$ is zero for $z<z_{0} \approx 1.3585$, and monotonously grows for $z>z_{0}$, rapidly approaching the asymptotic constant value, $f_{a s} \approx 1.1478$, for $z \gg z_{0}$. Then the Ioffe-Regel-Mott criterion, Eq. (4.27), at $\varepsilon$ so close to $\varepsilon_{\max }$ that $k_{\varepsilon} r_{\varepsilon} \gg z_{0}$, is expressed as

$$
\varepsilon_{\max }-\varepsilon \gg \frac{c^{2} \gamma^{2}}{\varepsilon_{\max }-\varepsilon_{0}} \frac{r_{\varepsilon}^{2}}{a^{2}},
$$

and this would result in a (concentration independent) estimate for the range of extended states within the impurity band,

$$
\varepsilon_{\max }-\varepsilon \gg \Gamma_{0}=\frac{\left(v \varepsilon_{0}\right)^{3 / 2}}{a k_{\mathrm{F}}} \sqrt{\frac{2 \pi \rho_{\mathrm{F}}}{1+v^{2}}},
$$

and its comparison with the full extension of this band, $\varepsilon_{\max }-\varepsilon_{\min }=c \gamma^{2}(1+$ $\left.v^{2}\right) /\left(v^{2} \Delta\right)$, would suggest possibility for such extended states to really exist if the impurity concentration surpass the characteristic (low) value:

$$
c \gg c_{0}=\frac{\left(\pi \rho_{\mathrm{F}} \varepsilon_{0}\right)^{3 / 2}}{a k_{\mathrm{F}}} \sqrt{\frac{2 v}{1+v^{2}}} .
$$




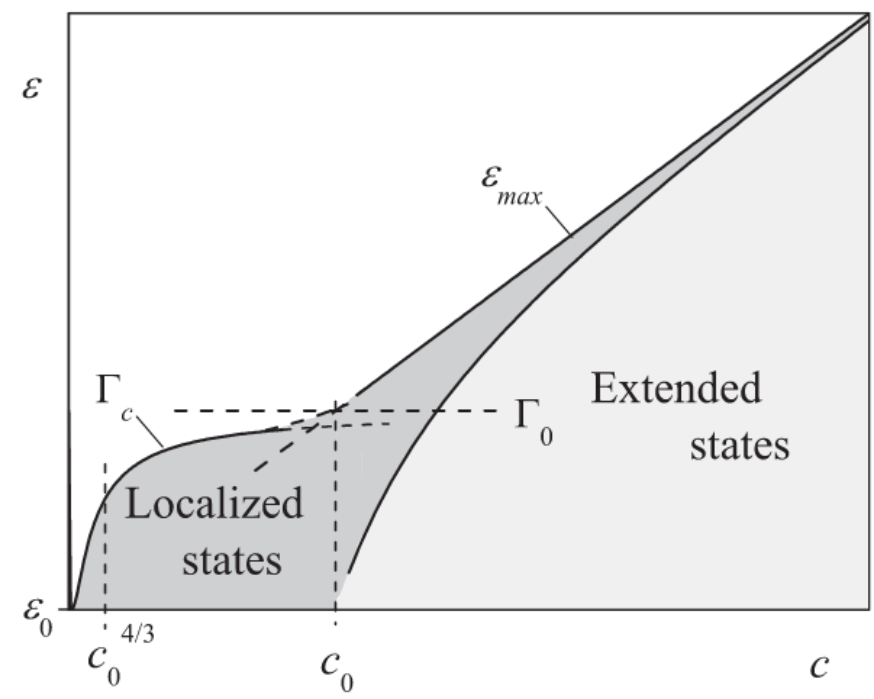

Figure 4.7: Structure of the energy spectrum near the impurity level in function of impurity concentration.

For typical values of $\rho_{\mathrm{F}}^{-1} \sim 2 \mathrm{eV}, a k_{\mathrm{F}} \sim 1$, and $\Delta \sim 10 \mathrm{meV}$ in LaOFeAs system, $[147,151,170]$, and supposing a plausible impurity perturbation $v \sim 1$, we estimate $c_{0} \approx 8 \cdot 10^{-4}$, manifesting important impurity effects already at their very low content.

However, the right-hand side of Eq. (4.34) vanishes at $k_{\varepsilon} r_{\varepsilon}<z_{0}$, which occurs beyond the vicinity of the band top:

$$
\varepsilon_{\max }-\varepsilon>\Gamma_{0}\left(\frac{c_{0}}{c}\right)^{3}
$$

Under the condition of Eq. (4.36), this vicinity is even more narrow than $\Gamma_{0}$ obtained with Eq. (4.35), defining the true, even wider, range of extended states.

Otherwise, for $c \ll c_{0}$, the impurity band does not exist, then we analyze the energy range near the impurity level with the nonrenormalized GE and write the approximate criterion for its convergence as $c\left|B^{0}\right| \ll 1$. This calculation is done in a similar way as before but replacing the interaction function, Eq. (4.29), by its nonrenormalized version: 


$$
A_{r}^{0}(\varepsilon) \approx \sqrt{R_{\varepsilon} / r} \mathrm{e}^{-r / r_{0}} \cos k_{\mathrm{F}} r
$$

with $k_{\mathrm{F}} R_{\varepsilon}=2 \pi\left(\varepsilon_{0} /\left|\varepsilon-\varepsilon_{0}\right|\right)^{2}$ and $k_{\mathrm{F}} r_{0}=2 \varepsilon_{\mathrm{F}} / \xi_{0}$. Then the above GE convergence criterion is assured beyond the following vicinity of impurity level:

$$
\left|\varepsilon-\varepsilon_{0}\right| \gg \Gamma_{c}=\Gamma_{0} \exp \left(-c_{0}^{4 / 3} / c\right),
$$

defining the range of its broadening due to interimpurity interactions. The DOS function for localized states can be only estimated by the order of magnitude within this range, but outside is given by

$$
\begin{aligned}
& \rho_{l o c}(\varepsilon) \approx \frac{c^{2}}{c_{0}^{4 / 3}\left|\varepsilon-\varepsilon_{0}\right|}, \text { for } \Gamma_{c} \ll\left|\varepsilon-\varepsilon_{0}\right| \ll \Gamma_{0}, \\
& \rho_{l o c}(\varepsilon) \approx \frac{c^{2} \varepsilon_{0}^{4}}{\left|\varepsilon-\varepsilon_{0}\right|^{5}}, \quad \text { for } \quad \Gamma_{0} \ll\left|\varepsilon-\varepsilon_{0}\right| .
\end{aligned}
$$

Notably, the total number of states near the impurity level is $\int \rho_{l o c}(\varepsilon) d \varepsilon \sim c$, like that of extended states in the impurity band with Eq. (4.26). A summary of evolution of this area of quasiparticle spectrum as a function of the impurity concentration is shown in Fig. 4.7.

\section{Impurity Effects on SC Characteristics}

The above results on the quasiparticle spectrum in a disordered SC system can be used immediately for calculation of impurity effects on its observable characteristics.

Thus the fundamental SC order parameter $\Delta$ is estimated from the modified gap equation,

$$
\lambda^{-1}=\int_{0}^{\varepsilon_{\mathrm{D}}} \rho(\varepsilon) d \varepsilon
$$

where $\lambda=\rho_{\mathrm{F}} V_{S C}$ is the (small) dimensionless SC pairing constant and the Debye energy $\varepsilon_{\mathrm{D}}$ restricts the energy range of its action. In absence of impurities, $c=0$, using the BCS DOS in this equation leads straightforwardly to the known result for its nonperturbed value $\Delta_{0}, \lambda^{-1}=\operatorname{arcsinh}\left(\varepsilon_{\mathrm{D}} / \Delta_{0}\right)$ and thus to $\Delta_{0} \approx \varepsilon_{\mathrm{D}} \mathrm{e}^{-1 / \lambda}$. 
For finite $c$, the total DOS is combined from the contributions from the shifted main band $\rho_{b}$, Eq. (4.25), and from the impurity band (or level) $\rho_{i}$ (or $\rho_{l o c}$ ), Eq. (4.26) [or (4.40)]. The latter contribution is $\sim c$, according to the previous discussion, defining a small correction beside $\lambda^{-1} \gg 1$. But a much stronger $c$-dependent correction comes from the modified main band:

$$
\begin{aligned}
\int_{\Delta_{c}}^{\varepsilon_{\mathrm{D}}} \rho_{b}(\varepsilon) d \varepsilon & \approx \operatorname{arcsinh} \frac{\varepsilon_{\mathrm{D}}}{\Delta_{c}} \\
& -c \gamma^{2} \int_{\Delta_{c}}^{\varepsilon_{\mathrm{D}}} \frac{d \varepsilon}{\left(\varepsilon-\varepsilon_{0}\right)^{2} \sqrt{\Delta_{c}^{2}-\varepsilon^{2}}} .
\end{aligned}
$$

For $\varepsilon_{\mathrm{D}} \gg \Delta_{c}$, the last integral is well approximated by

$$
c \gamma^{2} \int_{\Delta_{c}}^{\infty} \frac{d \varepsilon}{\left(\varepsilon-\varepsilon_{0}\right)^{2} \sqrt{\Delta_{c}^{2}-\varepsilon^{2}}}=\frac{c \gamma^{2}}{\Delta_{c}^{2}} F\left(\frac{\Delta_{c}}{\varepsilon_{0}}\right),
$$

with the function

$$
F(z)=z \frac{\sqrt{z^{2}-1}+z \arccos (-1 / z)}{\left(z^{2}-1\right)^{3 / 2}} .
$$

This $F$ diverges at $z \rightarrow 1$, but actually its argument,

$$
\Delta_{c} / \varepsilon_{0}=\sqrt{1+v^{2}}\left(1+c / c_{1}\right), \quad \text { with } \quad c_{1}=\pi \rho_{\mathrm{F}} \Delta / v,
$$

is always above unity. Neglecting the small $\rho_{i}$ contribution in Eq. (4.41) and taking account of the BCS relation $\lambda^{-1}=\operatorname{arcsinh}\left(\varepsilon_{\mathrm{D}} / \Delta_{0}\right)$, we express the gap equation as

$$
\operatorname{arcsinh} \frac{\Delta_{c}-\Delta_{0}}{\Delta_{0}} \approx \frac{c v^{2}}{c_{1}\left(1+v^{2}\right)} F\left(\Delta_{c} / \varepsilon_{0}\right) .
$$

Its approximate solution for $c \ll c_{1}$, together with the relation $\Delta_{c} / \Delta=$ $1+c /\left[c_{1}\left(1+v^{2}\right)\right]$, lead to the desired expression for the perturbed SC order parameter $\Delta$ :

$$
\frac{\Delta}{\Delta_{0}} \approx 1-\frac{c}{c_{1}} \frac{1+v^{2} F\left[\sqrt{1+v^{2}}\left(1+c / c_{1}\right)\right]}{1+v^{2}}
$$


that rapidly decays with impurity concentration and would vanish at

$$
c=c_{1} \frac{1+v^{2}}{1+v^{2} F\left[\sqrt{1+v^{2}}\left(1+c / c_{1}\right)\right]} .
$$

The latter equality defines in fact defines a certain equation for $c$ and its solution, e.g., for the above choice of $v=1$, is $c \approx 0.5 c_{1} \approx 6 \cdot 10^{-3}$. However, such concentrations would already correspond to an impurity band as wide as the gap itself; this goes beyond the validity of the above derivation and requires special treatment (to be done elsewhere).

To study another important dependence, that of the SC transition temperature $T_{c}$ on the concentration $c$, one must, strictly speaking, extend the above GF techniques for finite temperatures, but a very simple estimate can be done, supposing that the BCS relation $\Delta / T_{c} \approx 1.76$ still holds in the presence of impurities. Then the right-hand side of Eq. (4.42) would also describe the decay of $T_{c} / T_{c 0}$.

It is of interest to compare the present results with the known AbrikosovGor'kov solution for BCS SC with paramagnetic impurities in the Born approximation. $[171,172]$ In that approximation, the only perturbation parameter is the (constant) quasiparticle lifetime $\tau$. In our framework, the $\tau^{-1}$ can be related to $\operatorname{Im} \Sigma(\varepsilon)$ at a proper choice of energy, $\varepsilon \sim|\Delta-\varepsilon| \sim \Delta$. Then, in the self-consistent $T$-matrix approximation, [22] we estimate $\tau^{-1} \sim c \Delta / c_{1}$, which leads to the relation $\tau T_{c} \sim c_{1} / c$, reaching, at $c \gtrsim c_{1}$, qualitative agreement with the Abrikosov-Gor'kov universal criterion for complete SC suppression $\tau T_{c}<0.567$ (though in our case this criterion is not universal and depends yet on the perturbation parameter $v$ ).

Also, a notable impurity effect on the London penetration depth $\lambda_{\mathrm{L}} \sim n_{s}^{1 / 2}$ is expected, as follows from the temperature dependence of the superfluid density:

$$
\begin{aligned}
n_{s}(T) & =\int_{0}^{\infty} \frac{\rho(\varepsilon) d \varepsilon}{e^{\varepsilon / k_{\mathrm{B}} T}+1} \\
& \approx \frac{c}{e^{\varepsilon_{0} / k_{\mathrm{B}} T}+1}+\left(1-\frac{c \gamma^{2}}{\Delta^{2}-\varepsilon_{0}^{2}}\right) n_{s}^{0}(T) .
\end{aligned}
$$

Compared to its unperturbed value in the pure SC system, 


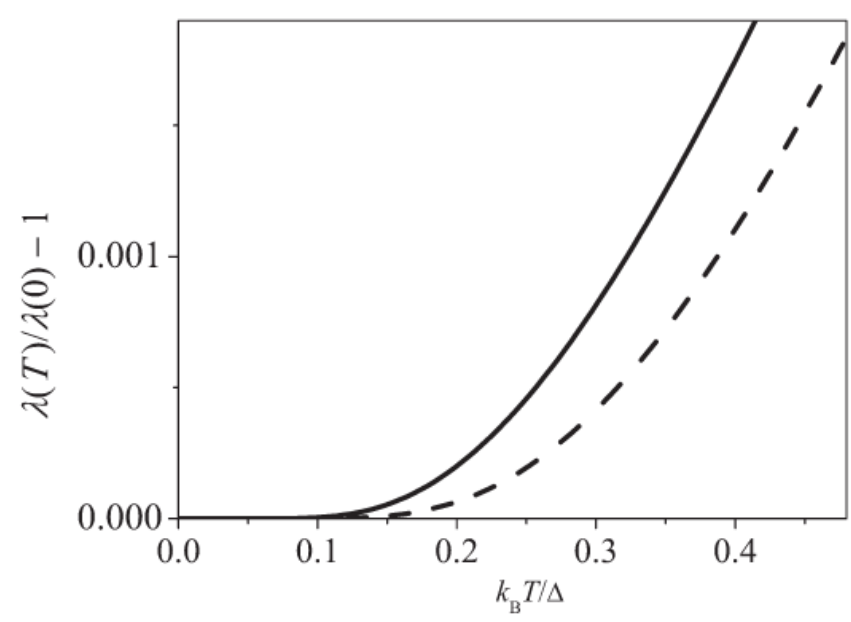

Figure 4.8: Low-temperature decay of the London penetration depthdifference for an SC with impurities (solid line) is slower than that inthe absence of impurities (dashed line).

$$
\begin{aligned}
n_{s}^{0}(T) & =\rho_{\mathrm{F}} \int_{\Delta}^{\infty} \frac{\varepsilon d \varepsilon}{\left(e^{\varepsilon / k_{\mathrm{B}} T}+1\right) \sqrt{\varepsilon^{2}-\Delta^{2}}} \\
& \approx \pi \rho_{\mathrm{F}} \sqrt{\frac{k_{\mathrm{B}} T \Delta}{2}} e^{-\Delta / k_{\mathrm{B}} T},
\end{aligned}
$$

a considerable slowing-down of the low-temperature decay of the characteristic difference $\lambda(T) / \lambda(0)-1$ is displayed (Fig. ??), in reasonable agreement with recent experimental observations for SC ferropnictides under doping. [173]

Finally, a similar analysis can be applied for the impurity effect on the electronic specific heat in the SC state, whose dependence on inverse temperature $\beta=1 / k_{\mathrm{B}} T$ is represented as

$$
C(\beta)=\frac{\partial}{\partial T} \int_{0}^{\infty} \frac{\rho(\varepsilon) d \varepsilon}{\mathrm{e}^{\beta \varepsilon}+1}
$$

and naturally divided in two characteristic contributions, $C=C_{i}+C_{b}$, from $\rho_{i}$ and $\rho_{b}$ states:

$$
C_{i}(\beta) \approx k_{\mathrm{B}} c\left[\frac{\beta \varepsilon_{0}}{2 \cosh \left(\beta \varepsilon_{0} / 2\right)}\right]^{2}
$$




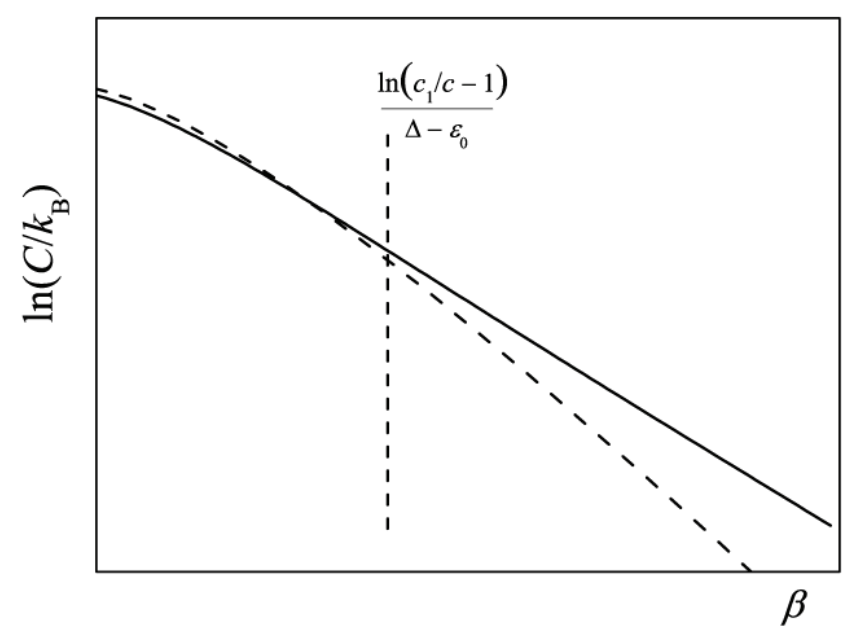

Figure 4.9: Temperature behavior of specific heat for a SC with impurities presents a crossover from $\beta \Delta$ exponent (dashed line) to $\beta \varepsilon_{0}$ at low enough temperature (high enough $\left.\beta=1 / k_{\mathrm{B}} T\right)$.

and

$$
C_{b}(\beta) \approx k_{\mathrm{B}}\left(c_{1}-c\right) v\left(\beta \Delta_{c}\right)^{3 / 2} \exp \left(-\beta \Delta_{c}\right)
$$

The resulting function $C(\beta)$ deviates from the known low temperature behavior $C_{0}(\beta) \sim \exp (-\beta \Delta)$ for a nonperturbed SC system at $\beta>\ln \left(c_{1} / c-1\right) /\left(\Delta-\varepsilon_{0}\right)$, where the characteristic exponent is changed to a slower $\sim \exp \left(-\beta \varepsilon_{0}\right)$ as shoen in Fig. 4.9.

The same approach can be used for calculation of other observable characteristics for the SC state under impurity effects, e.g., heat conductivity, differential conductivity for scanning tunneling spectroscopy, and absorption coefficient for far-infrared radiation, although these issues are beyond the scope of this work. 


\section{Conclusions}

In summary, the GF analysis of quasiparticle spectra in an SC ferropnictide with impurities of the simplest (local and nonmagnetic) perturbation type permits us to describe the formation of impurity localized levels within the SC gap and, with increasing impurity concentration, their evolution to specific bands of extended quasiparticle states, approximately described by the quasimomentum but mainly supported by the impurity centers. Explicit dispersion laws and DOS are obtained for the modified main bands and impurity bands.Further specification of the nature of all the states in different energy ranges within the SC gap is obtained through analysis of different types of GEs for a self-energy matrix, revealing a complex oscillatory structure of indirect interactions between impurity centers and, after their proper summation,resulting in criteria for crossovers between localized and extended states. The developed spectral characteristics are applied to the prediction of several observable impurity effects. The proposed treatment can be further adapted for analysis of more involved types of impurity perturbations in SC ferropnictides, including magnetic and nonlocal perturbations. 
Chapter 5

\section{Impurity effects on electronic transport in ferropnictide superconductors}




\title{
Impurity effects on electronic transport in ferropnictide superconductors
}

\author{
Y. G. Pogorelov, ${ }^{1}$ M. C. Santos, ${ }^{2}$ and V. M. Loktev ${ }^{3}$ \\ ${ }^{1}$ IFIMUP-IN, Departamento de Física, Universidade do Porto, Porto, Portugal \\ ${ }^{2}$ Departamento de Física, Universidade de Coimbra, R. Larga, Coimbra 3004-535, Portugal \\ ${ }^{3}$ Bogolyubov Institute for Theoretical Physics, NAS of Ukraine, 14b Metrologichna Street, 03143 Kiev, Ukraine \\ (Received 19 August 2013; revised manuscript received 15 December 2013; published 31 December 2013) \\ Effects of impurities and disorder on transport properties by electronic quasiparticles in superconducting iron \\ pnictides are theoretically considered. The most prominent new features compared to the case of pure material \\ should appear at high enough impurity concentration when a specific narrow band of conducting quasiparticle \\ states can develop within the superconducting gap, around the position of localized impurity level by a single \\ impurity center. The predicted specific threshold effects in the frequency-dependent optical conductivity and \\ temperature-dependent thermal conductivity and also in Seebeck and Peltier coefficients can have interesting \\ potentialities for practical applications.
}

DOI: 10.1103/PhysRevB.88.224518

PACS number(s): 74.70.Xa, 74.62.Dh, 74.62.En

\section{INTRODUCTION}

A considerable interest in actual research of superconductivity (SC) with high critical temperature is focused on the family of doped ferropnictide compounds ${ }^{1,2}$ and one of their notable distinctions from "old" BCS superconductors and more recent doped perovskite systems consists in the possibility for a peculiar, so-called extended $s$-wave symmetry of superconducting order parameter which changes its sign between electron and hole segments of the Fermi surface. ${ }^{3}$ This additional property permits avoiding the fundamental limitation by the Anderson theorem ${ }^{4}$ for nonmagnetic impurities to produce localized impurity levels within the superconducting band gap..$^{5,6}$ At finite, but low enough, impurity concentration, such levels are expected to give rise to some resonance effects like those well studied in semiconductors at low doping concentrations. ${ }^{7}$ Analogous effects in superconductors were theoretically predicted and experimentally discovered for magnetic impurities, either in BCS systems ${ }^{8-10}$ and in the two-band $\mathrm{MgB}_{2}$ system. ${ }^{11,12}$ In all those cases, the breakdown of the Anderson theorem is only due to the breakdown of the spin-singlet symmetry of an $s$-wave Cooper pair by a spin-polarized impurity, and the main physical interest of the considered case of SC iron pnictides from the point of view of disorder in general is the possibility for pair breaking even in nonmagnetic impurity ${ }^{13-15}$ states and for related localized in-gap states. ${ }^{16-19}$ This theoretical prediction was confirmed by the observations of various effects from localized impurity states, for instance, in the superfluid density (observed through the London penetration length), ${ }^{20,21}$ transition critical temperature, ${ }^{22,23}$ and electronic specific heat, ${ }^{24}$ all mainly due to an emerging spike of electronic density of states against its zero value in the initial band gap.

But it is also known that indirect interactions between random impurity centers of a certain type (the so-called deep levels at high enough concentrations) in doped semiconductors can lead to formation of collective bandlike states. ${ }^{25,26}$ This corresponds to the Anderson transition in a general disordered system, ${ }^{27}$ and the emerging new band of quasiparticles in the spectrum can essentially change thermodynamics and transport in the doped material. ${ }^{28}$ An intriguing possibility for similar banding of impurity levels within the SC gap $^{29,30}$ was recently discussed for the doped ferropnictides. ${ }^{31}$ The present work is aimed at a more detailed analysis of the bandlike impurity states, focused on their observable effects that cannot be produced by localized impurity states. We use the specific form of Green's functions for superconducting quasiparticles derived in the previous work $\mathrm{k}^{31}$ in the general Kubo-Greenwood formalism ${ }^{32}$ to obtain the temperature and frequency dependencies of optical and thermal conductivity and also of thermoelectric coefficients. These results are compared with the available experimental data and some suggestions are made on possible practical applications of such impurity effects.

\section{GREEN'S FUNCTIONS FOR DISORDERED SC FERROPNICTIDE}

We begin with a brief summary of the Green's function (GF) description of the electronic spectrum in LaOFeAs with impurities (not necessarily dopants) using the minimal coupling model ${ }^{17,33}$ for the nonperturbed Hamiltonian. It considers only 2 types of local Fe orbitals, $d_{x z}$ (or $x$ ) and $d_{y z}$ (or $y$ ), on sites of a square lattice with lattice parameter $a$ and 4 hopping parameters between nearest neighbors (NNs) and next-nearest neighbors (NNNs): (i) $t_{1}$ for $x x$ or $y y$ NNs along their orientations, and $t_{2}$ across them, and (ii) $t_{3}$ for $x x$ or $y y \mathrm{NNNs}$, and $t_{4}$ for $x y$ NNNs. The resulting band Hamiltonian is diagonal in quasimomentum $\mathbf{k}$ and $\operatorname{spin} \sigma$, but nondiagonal with respect to the orbital indices of the 2-spinors $\psi(\mathbf{k}, \sigma)=\left(x_{\mathbf{k}, \sigma}, y_{\mathbf{k}, \sigma}\right):$

$$
H_{t}=\sum_{\mathbf{k}, \sigma} \psi(\mathbf{k}, \sigma) \hat{h}(\mathbf{k}) \psi(\mathbf{k}, \sigma) .
$$

Here the energy matrix in orbital basis is expanded in Pauli matrices $\hat{\sigma}_{i}: \hat{h}(\mathbf{k})=\varepsilon_{+, \mathbf{k}} \hat{\sigma}_{0}+\varepsilon_{-, \mathbf{k}} \hat{\sigma}_{3}+\varepsilon_{x y, \mathbf{k}} \hat{\sigma}_{1}$ with the energy factors $\varepsilon_{ \pm, \mathbf{k}}=\left(\varepsilon_{x, \mathbf{k}} \pm \varepsilon_{x, \mathbf{k}}\right) / 2$, and

$$
\begin{aligned}
\varepsilon_{x, \mathbf{k}} & =-2 t_{1} \cos a k_{x}-2 t_{2} \cos a k_{y}-4 t_{3} \cos a k_{x} \cos a k_{y}, \\
\varepsilon_{y, \mathbf{k}} & =-2 t_{1} \cos a k_{y}-2 t_{2} \cos a k_{x}-4 t_{3} \cos a k_{x} \cos a k_{y}, \\
\varepsilon_{x y, \mathbf{k}} & =-4 t_{4} \sin a k_{x} \sin a k_{y} .
\end{aligned}
$$




\title{
Impurity effects on electronic transport in ferropnictide superconductors
}

\author{
Y.G. Pogorelov, ${ }^{1}$ M.C. Santos, ${ }^{2}$ V.M. Loktev ${ }^{3}$ \\ ${ }^{1}$ IFIMUP-IN, Departamento de Física, Universidade do Porto, Porto, \\ Portugal, \\ ${ }^{2}$ Departamento de Física, Universidade de Coimbra, R. Larga, Coimbra, \\ 3004-535, Portugal, \\ ${ }^{3}$ Bogolyubov Institute for Theoretical Physics, NAN of Ukraine, 14b \\ Metrologichna str., 03143 Kiev, Ukraine
}

Effects of impurities and disorder on transport properties by electronic quasiparticles in superconducting iron pnictides are theoretically considered. The most prominent new features compared to the case of pure material should appear at high enough impurity concentration when a specific narrow band of conducting quasiparticle states can develop within the superconducting gap, around the position of localized impurity level by a single impurity center. The predicted specific threshold effects in the frequency-dependent optical conductivity and temperature-dependent thermal conductivity and also in Seebeck and Peltier coefficients can have interesting potentialities for practical applications.

\section{Introduction}

A considerable interest in actual research of superconductivity (SC) with high critical temperature is focused on the family of doped ferropnictide compounds $[140,141]$ and one of their notable distinctions from "old" BCS superconductors and more recent doped perovskite systems consists in possibility for a peculiar, so-called extended s-wave symmetry of superconducting order parameter which changes its sign between electron and hole segments of the Fermi surface. [149] This additional property permits to avoid the fundamental limitation by the Anderson theorem [113] for nonmagnetic impurities to produce localized impurity levels within the superconducting band gap. [164, 163] At finite, but low enough, impurity concentration, such levels are expected to give rise to some resonance effects like those well studied in semiconductors at low doping concentrations. [34] Analogous effects in superconductors were theoretically predicted and experimentally discovered for magnetic impurities, either in BCS systems $[174,175,176]$ and in the two-band $\mathrm{MgB}_{2}$ system. [177, 178] In all those cases, the breakdown of the Anderson theorem is only due to the breakdown of the spin-singlet symmetry of an $s$-wave Cooper pair by a spinpolarized impurity, and the main physical interest of the considered case of SC 
iron pnictides from the point of view of disorder in general is the possibility for pair breaking even in nonmagnetic impurity $[179,180,181]$ states and for related localized in-gap states.[160, 182, 183, 184] This theoretical prediction was confirmed by the observations of various effects from localized impurity states, for instance, in the superfluid density (observed through the London penetration length), $[173,185]$ transition critical temperature, $[186,187]$ and electronic specific heat, [188] all mainly due to an emerging spike of electronic density of states against its zero value in the initial band gap.

But it is also known that indirect interactions between random impurity centers of certain type (the so-called deep levels at high enough concentrations) in doped semiconductors can lead to formation of collective bandlike states. $[134,189]$ This corresponds to the Anderson transition in a general disordered system, [48] and the emerging new band of quasiparticles in the spectrum can essentially change thermodynamics and transport in the doped material. [169] An intriguing possibility for similar banding of impurity levels within the SC gap [115, 190] was recently discussed for the doped ferropnictides. [23] The present work is aimed on a more detailed analysis of the bandlike impurity states, focused on their observable effects that cannot be produced by localized impurity states. We use the specific form of Green's functions for superconducting quasiparticles derived in the previous work [23] in the general Kubo-Greenwood formalism [191, 192] to obtain the temperature and frequency dependencies of optical and thermal conductivity and also of thermoelectric coefficients. These results are compared with the available experimental data and some suggestions are made on possible practical applications of such impurity effects.

\section{Green Functions for Disordered SC Ferropnictide}

We begin from a brief summary of the Green's function (GF) description of electronic spectrum in LaOFeAs with impurities (not necessarily dopants) using the minimal coupling model $[159,160]$ for the non-perturbed Hamiltonian. It considers only 2 types of local Fe orbitals, $d_{x z}$ (or $x$ ) and $d_{y z}$ (or $y$ ), on sites of square lattice with lattice parameter $a$ and 4 hopping parameters between nearest neighbors (NNs) and next nearest neighbors (NNNs): (i) $t_{1}$ for $x x$ or $y y$ NNs along their orientations, and $t_{2}$ across them, and (ii) $t_{3}$ for $x x$ or $y y$ NNNs, and $t_{4}$ for $x y$ NNNs. The resulting band Hamiltonian is diagonal in quasimomentum $\mathbf{k}$ and spin $\sigma$, but non-diagonal with respect to the orbital indices of the 2 -spinors $\psi^{\dagger}(\mathbf{k}, \sigma)=\left(x_{\mathbf{k}, \sigma}^{\dagger}, y_{\mathbf{k}, \sigma}^{\dagger}\right)$ : 


$$
H_{t}=\sum_{\mathbf{k}, \sigma} \psi^{\dagger}(\mathbf{k}, \sigma) \hat{h}(\mathbf{k}) \psi(\mathbf{k}, \sigma)
$$

Here the energy matrix in orbital basis is expanded in Pauli matrices $\hat{\sigma}_{i}: \hat{h}(\mathbf{k})=$ $\varepsilon_{+, \mathbf{k}} \hat{\sigma}_{0}+\varepsilon_{-, \mathbf{k}} \hat{\sigma}_{3}+\varepsilon_{x y, \mathbf{k}} \hat{\sigma}_{1}$ with the energy factors $\varepsilon_{ \pm, \mathbf{k}}=\left(\varepsilon_{x, \mathbf{k}} \pm \varepsilon_{x, \mathbf{k}}\right) / 2$, and

$$
\begin{aligned}
\varepsilon_{x, \mathbf{k}} & =-2 t_{1} \cos a k_{x}-2 t_{2} \cos a k_{y}-4 t_{3} \cos a k_{x} \cos a k_{y} \\
\varepsilon_{y, \mathbf{k}} & =-2 t_{1} \cos a k_{y}-2 t_{2} \cos a k_{x}-4 t_{3} \cos a k_{x} \cos a k_{y}, \\
\varepsilon_{x y, \mathbf{k}} & =-4 t_{4} \sin a k_{x} \sin a k_{y} .
\end{aligned}
$$

It is readily diagonalized at passing from the orbital to subband basis: $\hat{h}_{b}(\mathbf{k})=$ $\hat{U}(\mathbf{k}) \hat{h}(\mathbf{k}) \hat{U}(\mathbf{k})^{\dagger}$, with the unitary matrix $\hat{U}(\mathbf{k})=\exp \left(-i \hat{\sigma}_{2} \theta_{\mathbf{k}} / 2\right)$ and $\theta_{\mathbf{k}}=$ $\arctan \left(\varepsilon_{x y, \mathbf{k}} / \varepsilon_{-, \mathbf{k}}\right)$. The resulting eigenenergies for electron and hole subbands are

$$
\varepsilon_{h, e}(\mathbf{k})=\varepsilon_{+, \mathbf{k}} \pm \sqrt{\varepsilon_{x y, \mathbf{k}}^{2}+\varepsilon_{-, \mathbf{k}}^{2}},
$$

and respective electron and hole segments of the Fermi surface are defined by the equations $\varepsilon_{e, h}(\mathbf{k})=\varepsilon_{\mathrm{F}}$. A reasonable fit to the LaOFeAs band structure by the more detailed LDA calculations [152] is attained with the parameter choice (in $\left|t_{1}\right|$ units) of $t_{1}=-1, t_{2}=1.3, t_{3}=t_{4}=-0.85$. [154]

The SC state of such multiband electronic system is suitably described in terms of "multiband -Nambu" 4-spinors $\Psi_{\mathbf{k}}^{\dagger}=\left(\alpha_{\mathbf{k}, \uparrow}^{\dagger}, \alpha_{-\mathbf{k}, \downarrow}, \beta_{\mathbf{k}, \uparrow}^{\dagger}, \beta_{-\mathbf{k}, \downarrow}\right)$ with the multiband spinor $\left(\alpha_{\mathbf{k}, \sigma}^{\dagger}, \beta_{\mathbf{k}, \sigma}^{\dagger}\right)=\psi^{\dagger}(\mathbf{k}, \sigma) \hat{U}^{\dagger}(\mathbf{k})$, by a $4 \times 4$ extension of the Hamiltonian Eq. (5.1) in the form:

$$
H_{s}=\sum_{\mathbf{k}, \sigma} \Psi_{\mathbf{k}}^{\dagger} \hat{h}_{s}(\mathbf{k}) \Psi_{\mathbf{k}}
$$

where the $4 \times 4$ matrix $\hat{h}_{s}(\mathbf{k})=\hat{h}_{b}(\mathbf{k}) \otimes \hat{\tau}_{3}+\Delta_{\mathbf{k}} \hat{\sigma}_{0} \otimes \hat{\tau}_{1}$ includes the Pauli matrices $\hat{\tau}_{i}$ acting on the Nambu (particle-antiparticle) indices in $\Psi$-spinors. The simplified form for the extended $s$-wave gap function takes constant values $\Delta_{\mathrm{k}}=\Delta$ on the electron segments and $\Delta_{\mathrm{k}}=-\Delta$ on the hole segments.

The observable values result from the (Fourier transformed) GF $4 \times 4$ matrices $\hat{G}_{\mathbf{k}, \mathbf{k}^{\prime}}=\left\langle\left\langle\Psi_{\mathbf{k}} \mid \Psi_{\mathbf{k}^{\prime}}^{\dagger}\right\rangle\right\rangle$, and for the nonperturbed system, Eq. (5.1), they are diagonal in quasimomentum: $\hat{G}_{\mathbf{k}, \mathbf{k}^{\prime}}=\delta_{\mathbf{k}, \mathbf{k}^{\prime}} \hat{g}_{\mathbf{k}}$ with 


$$
\begin{aligned}
\hat{g}_{\mathbf{k}} & =\frac{\varepsilon \hat{\tau}_{0}+\varepsilon_{e}(\mathbf{k}) \hat{\tau}_{3}+\Delta \hat{\tau}_{1}}{2 d_{e, \mathbf{k}}} \otimes \hat{\sigma}_{e} \\
& +\frac{\varepsilon \hat{\tau}_{0}+\varepsilon_{h}(\mathbf{k}) \hat{\tau}_{3}-\Delta \hat{\tau}_{1}}{2 d_{h, \mathbf{k}}} \otimes \hat{\sigma}_{h}
\end{aligned}
$$

$\hat{\sigma}_{e, h}=\left(\hat{\sigma}_{0} \pm \hat{\sigma}_{3}\right) / 2, d_{i, \mathbf{k}}=\varepsilon^{2}-\varepsilon_{i}^{2}(\mathbf{k})-\Delta^{2}$.

To simplify the treatment of impurity perturbations, the band structure is approximated to identical circular electron and hole Fermi segments of radius $k_{\mathrm{F}}$ around respective points $\mathbf{K}_{e, h}$ in the Brillouin zone and to similar linear dispersion of normal state quasiparticles near the Fermi level $\varepsilon_{\mathrm{F}}: \varepsilon_{e}(\mathbf{k})-\varepsilon_{\mathrm{F}}=$ $\hbar v_{\mathrm{F}}\left(\left|\mathbf{k}-\mathbf{K}_{e}\right|-k_{\mathrm{F}}\right)$ and $\varepsilon_{h}(\mathbf{k})-\varepsilon_{\mathrm{F}}=-\hbar v_{\mathrm{F}}\left(\left|\mathbf{k}-\mathbf{K}_{h}\right|-k_{\mathrm{F}}\right)$. Moreover, we shall describe the contributions of both segments to overall electronic properties by a single quasimomentum variable $\xi$ that identifies electron $\xi_{e}=\varepsilon_{e}(\mathbf{k})-\varepsilon_{\mathrm{F}}$ and hole $\xi_{h}=\varepsilon_{h}(\mathbf{k})-\varepsilon_{\mathrm{F}}$ ones.

Next, the Hamiltonian of the disordered SC system is chosen as $H=H_{s}+H_{i m p}$ including besides $H_{s}$, Eq. (5.3), the term due to non-magnetic impurities [164] on random sites $\mathbf{p}$ in Fe square lattice with an on-site energy shift $V$ (supposed positive without loss of generality). It is written in the multiband-Nambu spinor form as:

$$
H_{i m p}=\frac{1}{N} \sum_{\mathbf{p}, \mathbf{k}, \mathbf{k}^{\prime}} \mathrm{e}^{i\left(\mathbf{k}^{\prime}-\mathbf{k}\right) \cdot \mathbf{p}} \Psi_{\mathbf{k}}^{\dagger} \hat{V}_{\mathbf{k}, \mathbf{k}^{\prime}} \Psi_{\mathbf{k}^{\prime}}
$$

with the number $N$ of unit cells in the crystal and the $4 \times 4$ scattering matrix $\hat{V}_{\mathbf{k}, \mathbf{k}^{\prime}}=V \hat{U}^{\dagger}(\mathbf{k}) \hat{U}\left(\mathbf{k}^{\prime}\right) \otimes \hat{\tau}_{3}$. In presence of this perturbation, the GFs can be expressed in specific forms depending on whether the considered quasiparticle energy falls into the range of bandlike or localized states. Namely, for bandlike states, the momentum diagonal GF,

$$
\hat{G}_{\mathbf{k}}=\hat{G}_{\mathbf{k}, \mathbf{k}}=\left(\hat{g}_{\mathbf{k}}^{-1}-\hat{\Sigma}_{\mathbf{k}}\right)^{-1} \text {, }
$$

involves the self-energy matrix $\hat{\Sigma}_{\mathbf{k}}$ in the form of the so-called renormalized group expansion: [132]

$$
\hat{\Sigma}_{\mathbf{k}}=c \hat{T}\left(1+c \hat{B}_{\mathbf{k}}+\ldots\right) .
$$

This series in powers of impurity concentration $c$ begins from the ( $k$-independent) $T$ matrix, $\hat{T}=\hat{V}(1-\hat{G} \hat{V})^{-1}$. From the matrices $\hat{V}=\hat{V}_{\mathbf{k}, \mathbf{k}}=V \hat{\tau}_{3}$ and 
$\hat{G}=N^{-1} \sum_{\mathbf{k}} \hat{g}_{\mathbf{k}}=\pi \varepsilon \rho_{\mathrm{F}} \hat{\tau}_{0} / \sqrt{\Delta^{2}-\varepsilon^{2}}$ (with the Fermi density of states $\rho_{\mathrm{F}}$ and the henceforth omitted trivial factor $\hat{\sigma}_{0}$ ), the $T$-matrix explicit form is

$$
\hat{T}=\frac{V}{1+v^{2}} \frac{v \varepsilon \sqrt{\Delta^{2}-\varepsilon^{2}} \hat{\tau}_{0}-\left(\Delta^{2}-\varepsilon^{2}\right) \hat{\tau}_{3}}{\varepsilon^{2}-\varepsilon_{0}^{2}} .
$$

where $\varepsilon_{0}=\Delta / \sqrt{1+v^{2}}$ defines the in-gap impurity levels [160] through the dimensionless impurity perturbation parameter $v=\pi \rho_{\mathrm{F}} V$. Inside the gap, the $T$-matrix, Eq. (5.8), is a real function which can be approximated near the impurity levels $\pm \varepsilon_{0}$ as $\hat{T} \approx \gamma^{2}\left(\varepsilon \hat{\tau}_{0}-\varepsilon_{0} \hat{\tau}_{3}\right) /\left(\varepsilon^{2}-\varepsilon_{0}^{2}\right)$, with the effective coupling constant $\gamma^{2}=V \varepsilon_{0}\left(v \varepsilon_{0} / \Delta\right)^{2}$. In contrary, outside the gap it is dominated by its imaginary part: $\operatorname{Im} \hat{T}=\left(\gamma^{2} / v \varepsilon_{0}\right) \varepsilon \sqrt{\varepsilon^{2}-\Delta^{2}} /\left(\varepsilon^{2}-\varepsilon_{0}^{2}\right)$.

The next terms besides unity in the brackets of Eq. (5.7) describe the effects of indirect interactions between impurities, with $\hat{B}_{\mathbf{k}}$ related to pairs and the omitted terms to groups of three and more impurities. The series convergence defines the energy ranges of bandlike states, delimited by the Mott mobility edges $\varepsilon_{c}$. [169] Within the bandlike energy ranges, the self-energy matrix can be safely approximated by the $T$-matrix, $\hat{\Sigma}_{\mathbf{k}} \approx c \hat{T}$, and the dispersion laws for corresponding bands at given quasimomentum $\mathbf{k}$ are defined from the $\hat{G}_{\mathbf{k}}$ denominator:

$$
\begin{aligned}
D_{\mathbf{k}}(\varepsilon) & =\operatorname{det} \hat{G}_{\mathbf{k}}^{-1}(\varepsilon)=\tilde{d}_{e, \mathbf{k}}(\varepsilon) \tilde{d}_{h, \mathbf{k}}(\varepsilon) \\
& =\left(\tilde{\varepsilon}^{2}-\tilde{\xi}_{e}^{2}-\Delta^{2}\right)\left(\tilde{\varepsilon}^{2}-\tilde{\xi}_{h}^{2}-\Delta^{2}\right),
\end{aligned}
$$

with the renormalized energy and momenta forms

$$
\begin{gathered}
\tilde{\varepsilon}=\varepsilon\left(1-\frac{c V v}{1+v^{2}} \frac{\sqrt{\Delta^{2}-\varepsilon^{2}}}{\varepsilon^{2}-\varepsilon_{0}^{2}}\right), \\
\tilde{\xi}_{j}=\xi_{j}-\frac{c V}{1+v^{2}} \frac{\Delta^{2}-\varepsilon^{2}}{\varepsilon^{2}-\varepsilon_{0}^{2}} .
\end{gathered}
$$

The roots of the dispersion equation $\operatorname{Re} D_{\mathbf{k}}(\varepsilon)=0$ define up to 8 subbands: 4 of them with energies near the roots of the nonperturbed denominators $d_{j, \mathbf{k}}$ in the $e$ and $h$ segments can be called "principal" or $p r$ bands (they are similar to quasiparticles in the pure crystal); and the other 4, "impurity" or imp bands, with energies near $\pm \varepsilon_{0}$ in the same segments are only specific for disordered systems. The dispersion law for $p$ bands is presented in the $\xi$-scale as 


$$
\varepsilon_{p r}(\xi) \approx \sqrt{\xi^{2}+\Delta^{2}}
$$

and it only differs from the nonperturbed one by the finite linewidth $\Gamma(\varepsilon) \approx$ $c \operatorname{Im} \hat{T}$, so that the validity range of Eq. (5.10) defined from the known IoffeRegel-Mott criterion, $\xi d \varepsilon_{b} / d \xi \gtrsim \Gamma\left(\varepsilon_{b}(\xi)\right)$, [53, 169] is $\xi \gtrsim c /\left(\pi \rho_{\mathrm{F}}\right)$. This defines the mobility edge in closeness to the gap edge,

$$
\varepsilon_{c}-\Delta \sim c^{2} / c_{0}^{4 / 3} \Delta .
$$

Here $c_{0}=\left(\pi \rho_{\mathrm{F}} \varepsilon_{0}\right)^{3 / 2} /\left(a k_{\mathrm{F}}\right) \sqrt{2 v /\left(1+v^{2}\right)}$ is the characteristic impurity concentration such that the impurity bands emerge just at $c>c_{0}$. [23] Their dispersion (in $\xi$ ) for the exemplar case of positive energies and $e$ segment is approximated as

$$
\varepsilon_{i m p}(\xi) \approx \varepsilon_{0}+c \gamma^{2} \frac{\xi-\varepsilon_{0}}{\xi^{2}+\xi_{0}^{2}}
$$

The formal upper limit energy by Eq. (5.12), $\varepsilon_{+}=\varepsilon_{0}+c \gamma^{2} /\left[2\left(\Delta+\varepsilon_{0}\right)\right]$, is attained at $\xi=\xi_{+}=\varepsilon_{0}+\Delta$ and the lower limit $\varepsilon_{-}=\varepsilon_{0}-c \gamma^{2} /\left[2\left(\Delta-\varepsilon_{0}\right)\right]$ at $\xi_{-}=\varepsilon_{0}-\Delta$. But in fact, this dispersion law is only valid until the related mobility edges $\varepsilon_{c, \pm}$ whose onset near the $i$-band edges is due to the higher terms in the group expansion, Eq. (5.7), and amounts to

$$
\begin{aligned}
& \varepsilon_{+}-\varepsilon_{c,+} \sim\left(\varepsilon_{\max }-\varepsilon_{0}\right)\left(\frac{c_{0}}{c}\right)^{4}, \\
& \varepsilon_{c,-}-\varepsilon_{-} \sim\left(\varepsilon_{0}-\varepsilon_{\min }\right)\left(\frac{c_{0}}{c}\right)^{4} .
\end{aligned}
$$

These limitations restrict $\xi$ to beyond some vicinities of the extremal points: $\left|\xi-\xi_{ \pm}\right| \gtrsim \xi_{ \pm}\left(c_{0} / c\right)^{2}$ (narrow enough at $c \gg c_{0}$ ). Another limitation is that $\xi$ not be too far from these points: $\left|\xi-\xi_{ \pm}\right| \lesssim \xi_{ \pm}\left(c / c_{0}\right)^{4}$. A symmetric replica of Eq. (5.12) near $-\varepsilon_{0}$ at the $e$ segment is the impurity subband with the dispersion law $-\varepsilon_{i}(\xi)$. Yet two more impurity subbands near the $h$ segment are described in the unified $\xi$ frame by the inverted dispersion laws $\pm \varepsilon_{i m p}(-\xi)$. The overall composition of band-like states in this frame is shown in Fig. 5.1. It is also important to notice that the above-described in-gap impurity band structure is only justified until it is narrow enough compared to the SC gap $\Delta$ itself. From Eq. (5.12), this requires that the impurity concentration stays well below the upper critical value
\[ c_{1}=\pi \rho_{\mathrm{F}} \Delta \sqrt{1+v^{2}} \] 
which can amount about few percent. In what follows, the condition $c \ll c_{1}$ is presumed.

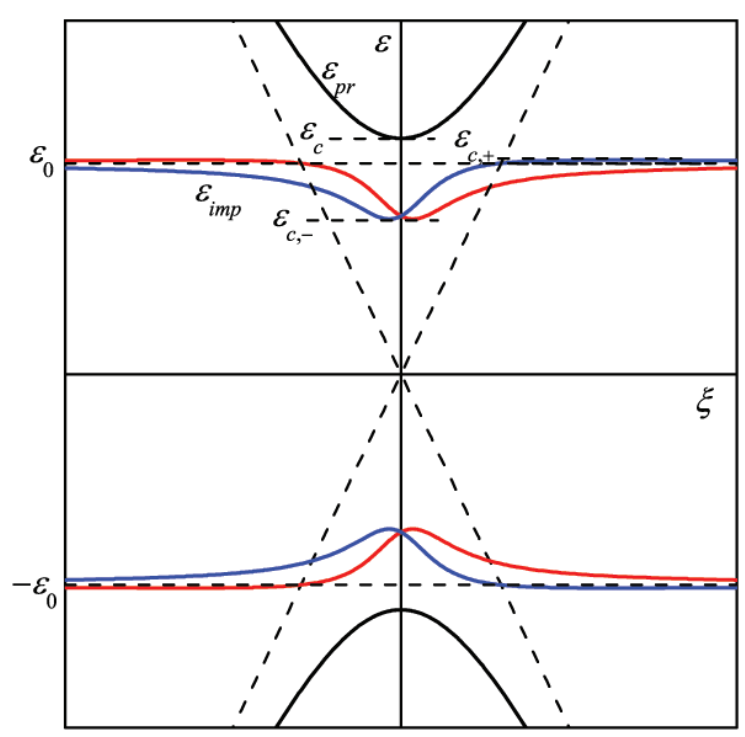

Figure 5.1: Dispersion laws in the modified quasiparticle spectrum of a SC ferropnictide with impurities. The impurity perturbation parameters were chosen as: $v=0.5, c_{0}=$ $1.3 \cdot 10^{-3}, c_{1}=1.7 \cdot 10^{-2}, c=4 \cdot 10^{-3}$. For compactness, the plot superimposes the blue lines for the in-gap impurity subbands near the electron-like pockets of the Fermi surface and red lines for those near the hole-like pockets.

At least, for $c<c_{0}$, all the in-gap states are localized and more adequately described by an alternative, the so called nonrenormalized group expansion of $\hat{G}_{\mathbf{k}}$ (though this case is beyond the scope of the present study) while the principal bands are still defined by Eqs. (5.10) and (5.11).

In-gap impurity states, either localized and bandlike, can produce notable resonance effects on various thermodynamical properties of disordered superconductors, as transition critical temperature, London penetration length, electronic specific heat, etc. [23] But besides that, other effects, only specific for 
new quasiparticle bands, can be expected on kinetic properties of the disordered material, while the localized impurity states should have practically no effect on them. Such phenomena can be naturally described in terms of the above indicated GF matrices as seen in what follows.

An important remark can yet be made on possible self-consistency corrections for the self-energy in Eq. (5.6) at the T-matrix level, as used in many known treatments of impurity effects (e.g., Ref. [181]). Such corrections can be also explicitly included in our approach but they will not change essentially the obtained bandlike spectra when the group expansion, Eq. (5.7), is converging.[193] Otherwise, if there is no such convergence and a MottAnderson transition from bandlike to localized states takes place, the very concept of self-consistency is not justified and, if still applied, can lead to spurious results as unphysical broadening of narrow impurity peaks in the spectrum (these caveats were recognized either in a general context of disordered solids39and specifically for impurity effects in superconductors [194]).

\section{Kubo-Greenwood Formalism for Multiband Superconductor}

The relevant kinetic coefficients for electronic processes in the considered disordered superconductor follow from the general Kubo-Greenwood formulation, [191, 192] adapted here to the specific multiband structure of Green's function matrices. Thus, one of the basic transport characteristics, the (frequency and temperature dependent) electrical conductivity, is expressed in this approach as

$$
\begin{aligned}
\sigma(\omega, T) & =\frac{e^{2}}{\pi} \int d \varepsilon \frac{f(\varepsilon)-f\left(\varepsilon^{\prime}\right)}{\omega} \int d \mathbf{k} v_{x}(\mathbf{k}, \varepsilon) v_{x}\left(\mathbf{k}, \varepsilon^{\prime}\right) \\
& \times \operatorname{Tr}\left[\operatorname{Im} \hat{G}_{\mathbf{k}}(\varepsilon) \operatorname{Im} \hat{G}_{\mathbf{k}}\left(\varepsilon^{\prime}\right)\right]
\end{aligned}
$$

for $\varepsilon^{\prime}=\varepsilon-\hbar \omega$ and the electric field applied along the $x$ axis. Besides the common Fermi occupation function $f(\varepsilon)=\left(\mathrm{e}^{\beta \varepsilon}+1\right)^{-1}$ with the inverse temperature $\beta=1 / k_{\mathrm{B}} T$, the above formula involves the generalized velocity function:

$$
\mathbf{v}(\mathbf{k}, \varepsilon)=\left(\hbar \frac{\partial \operatorname{Re} D_{\mathbf{k}}(\varepsilon)}{\partial \varepsilon}\right)^{-1} \nabla_{\mathbf{k}} \operatorname{Re} D_{\mathbf{k}}(\varepsilon) .
$$

This function is defined in the whole $\xi, \varepsilon$ plane in a way to coincide with the physical quasiparticle velocities for each particular band, Eqs. (5.9) and (5.12), along the corresponding dispersion laws: $\mathbf{v}\left(\mathbf{k}, \varepsilon_{j}(\mathbf{k})\right)=\hbar^{-1} \nabla_{\mathbf{k}} \varepsilon_{j}(\mathbf{k})=v_{j, \mathbf{k}}$, 
$j=p, i$. The conductivity resulting from Eq. (5.13) can be then used for calculation of optical reflectivity.

Other relevant quantities are the static (but temperature dependent) transport coefficients, as the heat conductivity:

$$
\kappa(T)=\frac{\hbar}{\pi} \int d \varepsilon \frac{\partial f(\varepsilon)}{\partial \varepsilon} \varepsilon^{2} \int d \mathbf{k}\left[v_{x}(\mathbf{k}, \varepsilon)\right]^{2} \operatorname{Tr}\left[\operatorname{Im} \hat{G}_{\mathbf{k}}(\varepsilon)\right]^{2},
$$

and the thermoelectric coefficients associated with the static electrical conductivity $\sigma(T) \equiv \sigma(0, T)$, the Peltier coefficient:[195]

$$
\begin{aligned}
\Pi(T) & =\frac{\hbar e}{\pi \sigma(0, T)} \int d \varepsilon \frac{\partial f(\varepsilon)}{\partial \varepsilon} \varepsilon \int d \mathbf{k}\left[v_{x}(\mathbf{k}, \varepsilon)\right]^{2} \\
& \times \operatorname{Tr}\left[\operatorname{Im} \hat{G}_{\mathbf{k}}(\varepsilon)\right]^{2}
\end{aligned}
$$

and the Seebeck coefficient $S(T)=\Pi(T) / T$. All these transport characteristics, though being relatively more complicated from the theoretical point of view than the purely thermodynamical quantities as, e.g., specific heat or London penetration length,[23] permit an easier and more reliable experimental verification and so could be of higher interest for practical applications of the considered impurity effects in the multiband superconductors.

It is worth recalling that the above formulas are only contributed by the bandlike states; that is, the energy arguments $\varepsilon, \varepsilon^{\prime}$ in Eqs. (5.14)-(5.17) are delimited by the relevant mobility edges. This is the main distinction of our approach from existing treatments of impurity effects on transport in ferropnictide superconductors using the $T$-matrix approximation to a solution like Eq. (5.6) for the whole energy spectrum,[196] even for its ranges where the very concept of velocity, as Eq. (5.15), ceases to be valid.

Next, we consider the particular calculation algorithms for the expressions, Eqs. (5.14), (5.16), and (5.17), beginning from the more involved case of dynamical conductivity, Eq. (5.14), and then reducing it to simpler static quantities, Eqs. (5.16) and (5.17). 


\section{Optical Conductivity}

The integral in Eq. (5.14) is dominated by the contributions from $\delta$-like peaks of the $\operatorname{Im} \hat{G}_{\mathbf{k}}(\varepsilon)$ and $\operatorname{Im} \hat{G}_{\mathbf{k}}\left(\varepsilon^{\prime}\right)$ matrix elements. These peaks arise from the above dispersion laws, Eqs. (5.9) and (5.11), thus restricting the energy integration to the bandlike ranges: $|\varepsilon|>\varepsilon_{c}$ for the $p$ bands and $\varepsilon_{c,-}<|\varepsilon|<\varepsilon_{c,+}$ for the $i$ bands. Regarding the occupation numbers $f(\varepsilon)$ and $f\left(\varepsilon^{\prime}\right)$ at reasonably low temperatures $k_{\mathrm{B}} T \ll \Delta, \varepsilon_{0}$, the most effective contributions correspond to positive $\varepsilon$ values, either from $p r$ or imp bands, and to negative $\varepsilon^{\prime}$ values from their negative counterparts, $p^{\prime}$ or $i^{\prime}$. There are three general kinds of such contributions: (i) $p r-p r^{\prime}$, due to transitions between the principal bands, similar to those in optical conductivity by the pure crystal (but with a slightly shifted frequency threshold: $\hbar \omega \geq 2 \varepsilon_{c}$ ), (ii) $p r-i m p^{\prime}$ (or $i m p-p r^{\prime}$ ), due to combined transitions between the principal and impurity bands within the frequency range $\hbar \omega \geq \varepsilon_{c}+\varepsilon_{c,-}$, and (iii) $i m p-i m p^{\prime}$, due to transitions between the impurity bands within a narrow frequency range of $2 \varepsilon_{c,-}<\hbar \omega<2 \varepsilon_{c,+}$. The frequency-momentum relations for these processes and corresponding peaks are displayed in Fig. ??. The resulting optical conductivity reads $\sigma(\omega, T)=\sum_{\nu} \sigma_{\nu}(\omega, T)$ with $\nu=p r-p r^{\prime}, i m p-i m p^{\prime}$, and $i m p-p r^{\prime}$.

For practical calculation of each contribution, the relevant matrix $\operatorname{Im} \hat{G}_{\mathbf{k}}(\varepsilon)$ (within the bandlike energy ranges) can be presented as $\operatorname{Im} \hat{G}_{\mathbf{k}}(\varepsilon)=\hat{N}(\varepsilon, \xi) \operatorname{Im}\left[D_{\mathbf{k}}(\varepsilon)^{-1}\right]$ where the numerator matrix,

$$
\hat{N}(\varepsilon, \xi)=\operatorname{Re}\left(\tilde{\varepsilon}+\tilde{\xi} \hat{\tau}_{3}+\Delta \hat{\tau}_{1}\right),
$$

is a smooth enough function while the peaks referred to above result from zeros of $\operatorname{Re} D_{\mathbf{k}}(\varepsilon)$. Now, the quasimomentum integration in Eq. (5.14) under the above-chosen symmetry of Fermi segments spells as $\int d \mathbf{k}=2\left(h v_{\mathrm{F}}\right)^{-1} \int d \varphi \int d \xi$ where the factor 2 accounts for identical contributions from $e$ and $h$ segments. The azimuthal integration contributes by the factor of $\pi$ (from $x$ projections of velocities) and the most important radial integration is readily done after expanding its integrand in particular pole terms:

$$
\begin{aligned}
v(\xi, \varepsilon) v\left(\xi, \varepsilon^{\prime}\right) & \operatorname{Tr} \quad\left[\operatorname{Im} \hat{G}(\xi, \varepsilon) \operatorname{Im} \hat{G}\left(\xi, \varepsilon^{\prime}\right)\right] \\
& =\sum_{\alpha} A_{\alpha}\left(\varepsilon, \varepsilon^{\prime}\right) \delta\left(\xi-\xi_{\alpha}\right)
\end{aligned}
$$

where $v(\xi, \varepsilon)=|\mathbf{v}(\mathbf{k}, \varepsilon)|$ and $\hat{G}\left(\xi, \varepsilon^{\prime}\right) \equiv \hat{G}_{\mathbf{k}}\left(\varepsilon^{\prime}\right)$ define the respective residues: 


$$
A_{\alpha}\left(\varepsilon, \varepsilon^{\prime}\right)=\pi v_{\alpha} v_{\alpha}^{\prime} \frac{\tilde{\varepsilon} \tilde{\varepsilon}^{\prime}+\tilde{\xi} \tilde{\xi}^{\prime}+\Delta^{2}}{\prod_{\beta \neq \alpha}\left(\xi_{\alpha}-\xi_{\beta}\right)}
$$

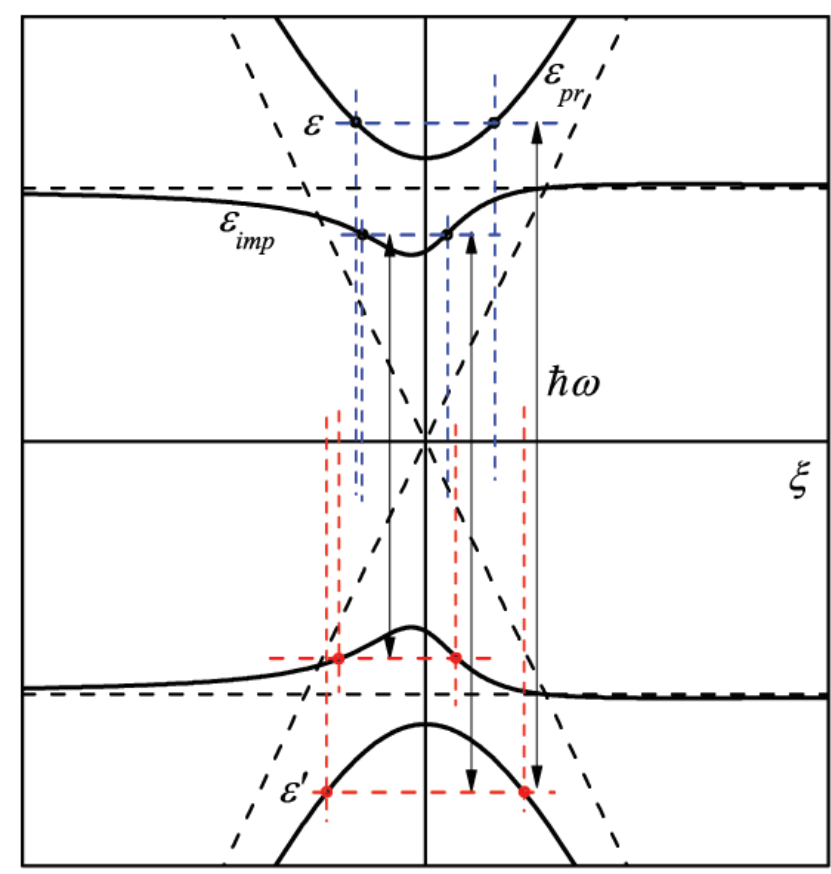

Figure 5.2: Configuration of the poles $\xi_{j}$ of GFs contributing to different types of optical conductivity processes over one part (electronic pocket) of the quasiparticles spectrum by Fig. 5.1.

Here $v_{\alpha} \equiv v\left(\varepsilon, \xi_{\alpha}\right), v_{\alpha}^{\prime} \equiv v\left(\varepsilon^{\prime}, \xi_{\alpha}\right)$, and the indices $\alpha, \beta$ run over all the poles of the two Green's functions. As follows from Eqs. (5.10) and (5.12) and seen in Fig. 5.2, there can be two such poles of $\hat{G}(\xi, \varepsilon)$ related to bandlike states 
with positive $\varepsilon$ and respective quasimomentum values denoted as $\xi_{1,2}(\varepsilon)$. For energies within the $p r$ band, $\varepsilon>\varepsilon_{c}$, they are symmetrical:

$$
\xi_{1,2}(\varepsilon) \approx \pm \sqrt{\varepsilon^{2}-\Delta^{2}}
$$

while within the $i m p$ band at $\varepsilon_{c,-}<\varepsilon<\varepsilon_{c,+}$, their positions are asymmetrical:

$$
\xi_{1,2}(\varepsilon) \approx \frac{c \gamma^{2} \mp 2 \varepsilon_{0} \sqrt{\left(\varepsilon_{+}-\varepsilon\right)\left(\varepsilon-\varepsilon_{-}\right)}}{2\left(\varepsilon-\varepsilon_{0}\right)}
$$

Notice also that, within the imp band, there is a narrow vicinity of $\varepsilon_{0}$ of $\sim c_{0}^{1 / 3}\left(c_{0} / c\right)^{3} \varepsilon_{0}$ width where only the $\xi_{1}$ pole by Eq. (5.22) is meaningful and the other contradicts to the Ioffe-Regel-Mott criterion (so that there are no bandlike states with that formal $\xi_{2}$ values in this energy range). Analogous poles of $\hat{G}\left(\xi, \varepsilon^{\prime}\right)$ at negative $\varepsilon^{\prime}$ are referred to as $\xi_{3,4}\left(\varepsilon^{\prime}\right)$ in what follows. Taking into account a non-zero $\operatorname{Im} D_{\mathbf{k}}(\varepsilon)$ [for the $i m p$ band, it is due to the nontrivial terms in the group expansion, Eq. (5.7)], each $\alpha$ th pole becomes a $\delta$-like peak with an effective linewidth $\Gamma_{\alpha}$ but this value turns to be essential (and will be specified) only at calculation of static coefficients like Eqs. (5.16) and (5.17).

Since four peaks in Eq. (5.19) for optical conductivity are typically well separated, the $\xi$-integration is trivially done considering them true $\delta$-functions; then the particular terms in $\sigma(\omega, T)$ follow as the energy integrals:

$$
\sigma_{\nu}(\omega, T)=2 e^{2} \int_{\varepsilon_{\nu,-}}^{\varepsilon_{\nu,+}} d \varepsilon \frac{f(\varepsilon)-f\left(\varepsilon^{\prime}\right)}{\omega} \sum_{\alpha=1}^{4} A_{\alpha}\left(\varepsilon, \varepsilon^{\prime}\right)
$$

where $\nu$ takes the values $p r-p r^{\prime}, i m p-p r^{\prime}$, or $i m p-i m p^{\prime}$ and the limits $\varepsilon_{\nu, \pm}$ should assure that both $\varepsilon$ and $\varepsilon^{\prime}$ are kept within the respective bandlike energy ranges.

Thus, in the $p r-p r^{\prime}$ term, the symmetry of the poles $\xi_{1,2}(\varepsilon)$ and $\xi_{3,4}\left(\varepsilon^{\prime}\right)$ by Eq. (5.21) and the symmetry of $p r$ and $p r^{\prime}$ bands themselves defines their equal contributions; then using simplicity of the generalized velocity function $v(\xi, \varepsilon)=\xi / \varepsilon$ and the nonrenormalized energy and momentum variables, $\tilde{\varepsilon} \rightarrow \varepsilon$, $\tilde{\xi} \rightarrow \xi$, the energy integration between the limits $\varepsilon_{p r-p r^{\prime},-}=\varepsilon_{c}$ and $\varepsilon_{p r-p r^{\prime},+}=$ $\hbar \omega-\varepsilon_{c}$ provides its explicit analytic form as $\sigma_{p r-p r^{\prime}}(\hat{\omega}, T)=\sigma_{p r-p r^{\prime}}(\omega, 0)-$ $\sigma_{p r-p r^{\prime}, T}(\omega)$. Here the zero-temperature limit value is 


$$
\begin{aligned}
\sigma_{p r-p r^{\prime}}(\omega, 0) & \approx \sigma_{0} \frac{2 \omega_{c}}{\omega^{2}}\left\{\sqrt{4 \omega^{2}-\omega_{c}^{2}} \ln \left[2 \frac{\omega\left(2 \omega-\omega_{c}\right)+\sqrt{\omega\left(\omega-\omega_{c}\right)\left(4 \omega^{2}-\omega_{c}^{2}\right)}}{\omega_{c}^{2}}-1\right]\right. \\
& \left.+2 \omega \ln \left[2 \frac{\omega-\sqrt{\omega\left(\omega-\omega_{c}\right)}}{\omega_{c}}-1\right]-2 \sqrt{\omega\left(\omega-\omega_{c}\right)}\right\}
\end{aligned}
$$

with the characteristic scale $\sigma_{0}=e^{2} / \Delta^{2}$ and simple asymptotics:

$$
\begin{aligned}
\sigma_{p r-p r^{\prime}}(\omega, 0) & \approx(2 / 3) \sigma_{0}\left(\omega / \omega_{c}-1\right)^{3 / 2}, \quad \omega-\omega_{c} \ll \omega_{c}, \\
\sigma_{p r-p r^{\prime}}(\omega, 0) \approx \sigma_{0}\left(32 \omega_{c} / \omega\right) \ln \left(2 \omega / \omega_{c}\right), & \omega \gg \omega_{c},
\end{aligned}
$$

with respect to the threshold frequency $\omega_{c}=2 \varepsilon_{c} / \hbar$, reaching the maximum value $\approx 1.19 \sigma_{0}$ at $\omega \approx 2.12 \omega_{c}$ as seen in Fig. 5.3. The (small) finitetemperature correction to the above value,

$$
\begin{aligned}
\sigma_{p r-p r^{\prime}, T}(\omega) & \approx \sigma_{0} \frac{2 \omega_{c}^{2} \mathrm{e}^{-\beta \Delta}}{\beta \hbar\left(\omega-\omega_{c}\right) \omega \sqrt{\Delta}}\left[\frac{\sqrt{\hbar \omega}}{\Delta}\left(1-\frac{F\left(\sqrt{\beta \hbar\left(\omega-\omega_{c}\right)}\right)}{\sqrt{\beta \hbar\left(\omega-\omega_{c}\right)}}\right)\right. \\
& \left.+\frac{\sqrt{2 \Delta}}{\hbar \omega-\Delta}\left(\frac{\sqrt{\pi}}{2} \frac{\operatorname{erf}\left(\sqrt{\beta \hbar\left(\omega-\omega_{c}\right)}\right)}{\sqrt{\beta \hbar\left(\omega-\omega_{c}\right)}}-\mathrm{e}^{-\beta \hbar\left(\omega-\omega_{c}\right)}\right)\right],(5 .
\end{aligned}
$$

involves the Dawson function $F(z)=\sqrt{\pi} \mathrm{e}^{-z^{2}} \operatorname{erf}(i z) /(2 i)$ and the error function $\operatorname{erf}(z)$. [197]

Calculation of the $i m p-p r^{\prime}$ term is more complicated since asymmetry of the $i m p$-band poles $\xi_{1,2}(\varepsilon)$ by Eq. (5.22) and their nonequivalence to the symmetric poles $\xi_{3,4}\left(\varepsilon^{\prime}\right)$ of the $p r^{\prime}$ band analogous to Eq. (5.21). More complicated expressions also define the generalized velocity function within the $i m p$-band range,

$$
\hbar v(\xi, \varepsilon)=\frac{c \gamma^{2}-\xi\left(\varepsilon-\varepsilon_{0}\right)}{\varepsilon\left(\varepsilon-\varepsilon_{0}-c \gamma^{2} / \varepsilon_{0}\right)},
$$

and the energy integration limits: $\varepsilon_{i m p-p r^{\prime},-}=\varepsilon_{c,-}$ and $\varepsilon_{i m p-p r^{\prime},+}=\min \left[\varepsilon_{c,+}, \hbar \omega-\right.$ $\left.\varepsilon_{c}\right]$. Then the function $\sigma_{i m p-p r^{\prime}}(\omega, T)$ follows from a numerical integration in Eq. (5.23) and, as seen in Fig. 5.3, it has a lower threshold frequency $\omega_{c}^{\prime}=\varepsilon_{c}+\varepsilon_{c,-}$ than the $p r-p r^{\prime}$ term. Above this threshold, it starts to grow linearly as $\sim\left(\omega / \omega_{c}^{\prime}-1\right) c^{5 / 2} c_{0}^{-5 / 3} \sigma_{0}$ and, for the impurity concentrations within the "safety range", $c \ll c_{1} \sim c_{0}^{2 / 3}$, becomes completely dominated by the $p r-p r^{\prime}$ function, Eq. (5.24) above its threshold $\omega_{c}$. 
Finally, the $i m p-i m p^{\prime}$ term is obtained with a similar numerical routine on Eq. (5.23), using Eq. (5.22) either for the poles $\xi_{1,2}(\varepsilon)$ by the $i m p$ band and for the $\xi_{3,4}\left(\varepsilon^{\prime}\right)$ by the $i m p^{\prime}$ band and Eq. (5.26) for respective generalized velocities, while the energy integration limits in this case are $\varepsilon_{i m p-i m p^{\prime},-}=\varepsilon_{c,-}$ and $\varepsilon_{i m p-i m p^{\prime},+}=\min \left[\varepsilon_{c,+}, \hbar \omega-\varepsilon_{c .-}\right]$. The resulting function $\sigma_{i m p-i m p^{\prime}}(\omega, T)$ occupies the narrow frequency band from $\omega_{i m p-i m p^{\prime,-}}=2 \varepsilon_{c,-} / \hbar$ to $\omega_{i m p-i m p^{\prime},+}=$ $2 \varepsilon_{c,+} / \hbar$ (Fig. 5.3) and its asymptotics near these thresholds and in the zerotemperature limit are obtained analytically as:

$$
\sigma_{i m p-i m p^{\prime}}(\omega, 0) \approx \sigma_{0} \frac{16 c^{7 / 2} \gamma^{7}}{3 \sqrt{2} \xi_{-}^{7}}\left(\frac{\omega-\omega_{-}}{\omega_{-}}\right)^{3 / 2}
$$

at $0<\omega-\omega_{-} \ll \omega_{-}$and a similar formula for $0<\omega_{+}-\omega \ll \omega_{+}$only differs from it by the change: $\xi_{-} \rightarrow \xi_{+}$and $\omega_{-} \rightarrow \omega_{+}$.

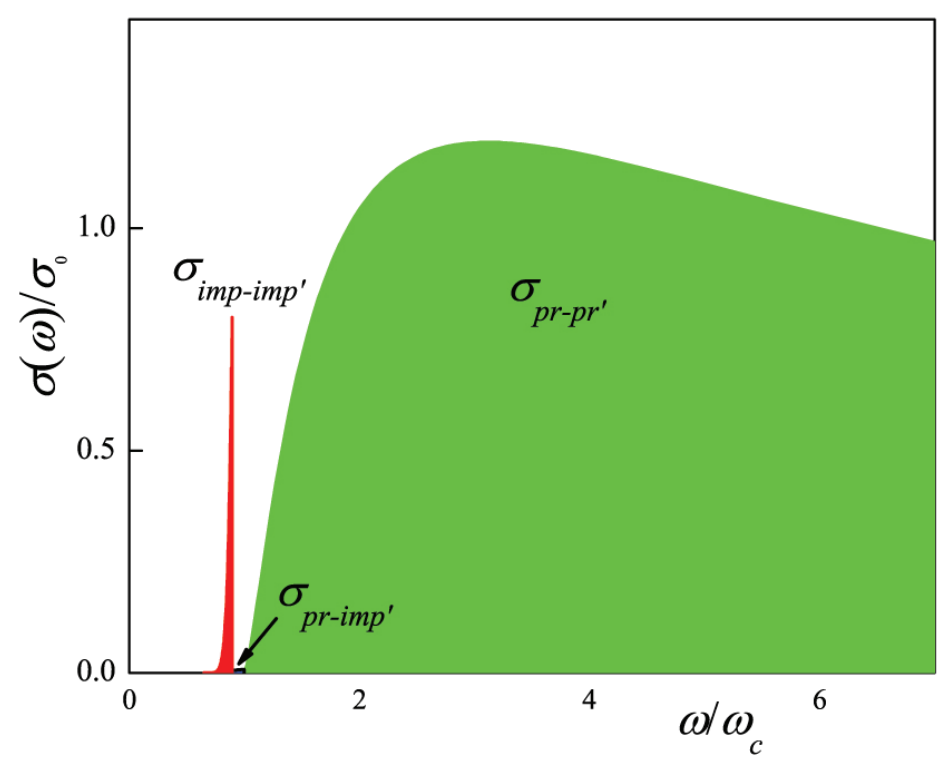

Figure 5.3: General picture of the optical conductivity showing three types of contributions. 
Then the maximum contribution by the $i m p-i m p^{\prime}$ term is estimated by extrapolation of the above asymptotics to the center of the impurity band: $\left|\omega-\omega_{ \pm}\right| \sim\left|\omega_{0}-\omega_{ \pm}\right|$, resulting in $\sigma_{i m p-i m p^{\prime}, \text { max }} \sim \sigma_{0} c^{5} c_{0}^{-10 / 3}\left(\xi_{+} / \xi_{-}\right)^{7 / 2}$. This estimate shows that the narrow $i m p-i m p^{\prime}$ peak of optical conductivity around $\omega \approx 2 \varepsilon_{0} / \hbar$ can, unlike the "combined" imp - pr' term, become as intense or even more so than the maximum of "principal" $p r-p r^{\prime}$ intensity, Eq. (5.24), if the small factor $\sim\left(c / c_{1}\right)^{5}$ is overweighted by the next factor $\left(\xi_{+} / \xi_{-}\right)^{7 / 2}$. The latter is only possible if the impurity perturbation is weak enough: $v \ll$ 1. Then the ratio $\xi_{+} / \xi_{-}$turns $\approx(2 / v)^{2} \gg 1$ and can really overweight the concentration factor if the impurity concentration $c$ reaches $\sim c_{1}(v / 2)^{7 / 5} \ll c_{1}$, that is quite realistic within the "safety" range $c \ll 1$. The overall picture of optical conductivity for an example of weakly coupled, $v=0.25$, impurities at high enough concentration $c=4 c_{0}$ is shown in Fig. 5.3. The expressed effect of "giant" optical conductivity by the in-gap impurity excitations could be compared with the well-known Rashba enhancement of optical luminescence by impurity levels at closeness to the edge of excitonic band [198] or with the huge impurity spin resonances in magnetic crystals, [132] but with a distinction that it appears here in a two-particle process instead of the above mentioned single-particle ones.

It should be underlined again that the considered impurity features in optical conductivity cannot be interpreted in a simplistic view of optical transitions between localized impurity states (or between these and principal band states)since the lack of mobility for localized states would prevent their contribution to the currents. This is only recovered at high enough impurity concentrations, $c \gtrsim c_{0}$, when the impurity state banding takes place.

\section{Static Kinetic Coefficients}

Now we can pass to the relatively simpler calculation of the kinetic coefficients in the static limit of $\omega \rightarrow 0$. To begin with, consider the heat conductivity, Eq. (5.16), where the momentum integration at coincidence of the above mentioned poles $\xi_{1.3}$ and $\xi_{2.4}$ is readily done using the general convolution formula,

$$
\int L_{\Gamma_{j}}\left(\xi-\xi_{j}\right) L_{\Gamma_{k}^{\prime}}\left(\xi-\xi_{k}^{\prime}\right) d \xi=L_{\Gamma_{j}+\Gamma_{k}^{\prime}}\left(\xi_{j}-\xi_{k}^{\prime}\right),
$$

for two Lorentzian fuctions $L_{\Gamma}(\xi)=\Gamma /\left(\xi^{2}+\Gamma^{2}\right)$, and in the limit of $\xi_{i}=\xi_{k}^{\prime}$ and $\Gamma_{j}=\Gamma_{k}^{\prime}$ obtaining simply $\left(2 \Gamma_{j}\right)^{-1}$, a "combined lifetime". This immediately leads to a Drude-like formula for heat conductivity as a sum of principal and impurity terms, $\kappa(T)=\kappa_{p r}(T)+\kappa_{i m p}(T)$, each of them given by 


$$
\begin{aligned}
\kappa_{p r}(T) & =\frac{\hbar\left(1+v^{2}\right)}{\pi c V v} \int_{\varepsilon_{c}}^{\infty} d \varepsilon \frac{\partial f(\varepsilon)}{\partial \varepsilon} \frac{\varepsilon\left(\varepsilon^{2}-\varepsilon_{0}^{2}\right)}{\sqrt{\varepsilon^{2}-\Delta^{2}}} \\
& \approx \frac{\hbar \rho_{\mathrm{F}} \Delta^{2}}{c} \sqrt{\frac{\pi \beta \Delta}{2}} \exp (-\beta \Delta)
\end{aligned}
$$

and

$$
\begin{aligned}
\kappa_{i m p}(T) & \approx \frac{\hbar}{\pi\left(\varepsilon_{c,+}-\varepsilon_{c,-}\right)}\left(\frac{c}{c_{0}}\right)^{4} \int_{\varepsilon_{c,-}}^{\varepsilon_{c,+}} d \varepsilon \frac{\partial f(\varepsilon)}{\partial \varepsilon} \varepsilon^{2} \\
& \approx \frac{\hbar}{\pi}\left(\frac{c}{c_{0}}\right)^{4} \beta \varepsilon_{0}^{2} \exp \left(-\beta \varepsilon_{0}\right) .
\end{aligned}
$$

Then the comparison of Eqs. (5.29) and (5.30) shows that the impurity contribution to the heat conductance $\kappa_{i m p}$ for impurity concentrations $c$ above the critical value $c_{0}$ turns to dominate over the principal contribution $\kappa_{p r}$ at all the temperatures (of course, below the critical transition temperature). Such strong impurity effect is combined from enhanced thermal occupation of impurity states and from their growing lifetime as $\sim c^{3}$ against the decreasing as $\sim 1 / c$ lifetime in the principal band.

Similar strong impurity effects should also follow for the static electric conductivity $\sigma(0, T)$ (see Ref. [195]) and for the thermoelectric Peltier and Seebeck coefficients, Eq. (5.17). All of them can be considered as fully due to the corresponding impurity contributions and the temperature dependencies of thermoelectric coefficients should be nonexponential: $\Pi(T) \approx \Pi(0)=$ constant, and $S(T) \approx \Pi(0) / T$, alike the nonperturbed case but at much higher level. Like the final note in the previous section, these predictions are only valid for impurity concentrations above the critical value, $c \gtrsim c_{0}$, while the system transport properties should stay almost nonaffected by impurities below this concentration, $c<c_{0}$. Fig. 5.4 demonstrates these differences between temperature dependencies of static conductivities and of thermoelectric coefficients for low and high concentrations of impurities at the choice of perturbation parameter as $v=1$. Such drastic changes of transport behavior are of interest for experimental verification in properly prepared samples of SC ferropnictides with controlled concentration of specific impurities. 


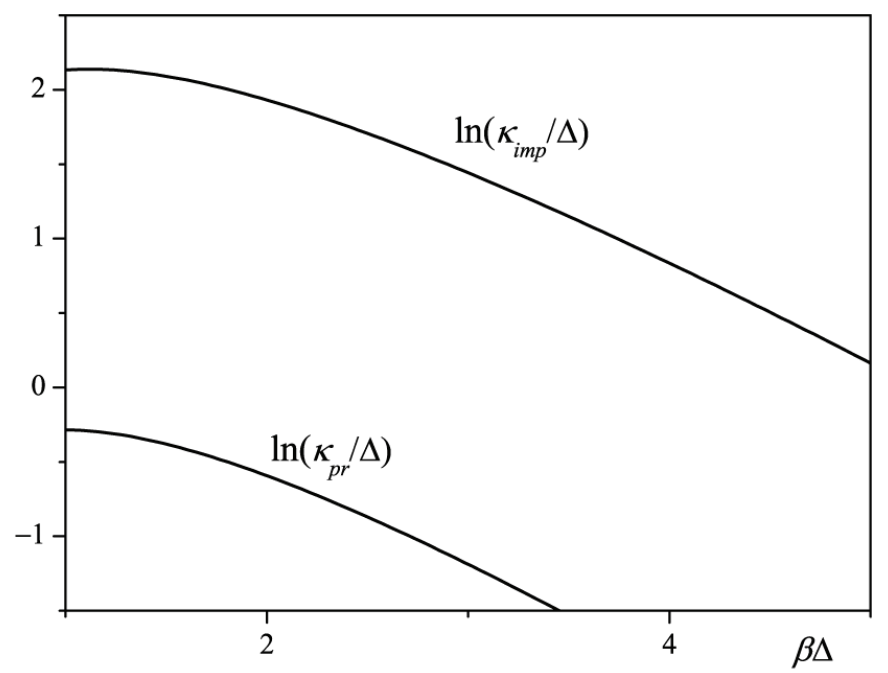

Figure 5.4: Logarithmic plots for two contributions to the heat conductivity shows domination of the impurity term at all the temperatures where SC itself exists. 


\section{Conclusive Remarks}

In conclusion, the essential modification of quasiparticle spectra in a SC ferropnictide with impurities of simplest (local and nonmagnetic) perturbation type is expected, consisting of formation of localized in-gap impurity states and their development into specific narrow bands of impurity quasiparticles at impurity concentration above a certain (quite low) critical value $c_{0}$ and leading to a number of effects in the system's observable properties. Besides the previously discussed thermodynamical effects, expected to appear at all impurity concentrations, which are due to either localized or bandlike impurity states, a special interest is seen in studying the impurity effects on electronic transport properties of such systems, only affected by the impurity bandlike states. It was shown above that the latter effects can be very strongly pronounced, either for high-frequency transport and for static transport processes. In the first case, the impurity effect is expected to most strongly be revealed in a narrow peak of optical conductance at its closeness to the edge of conductance band in nonperturbed crystal, resembling the known resonance enhancement of impurity absorption (or emission) processes near the edge of main quasiparticle band in normal systems; here it would be possible if the impurity perturbation were weak enough. The static transport coefficients at overcritical impurity concentrations are also expected to be strongly enhanced compared to those in a nonperturbed system, including the thermoelectric Peltier and Seebeck coefficients.

The above-presented simplest theoretical model can be extended to include either more realistic multiorbital structures of the initial ferropnictide system or more general type sof impurity perturbation on it (e.g., as extended centers considered earlier in $d$-wave cuprate systems [199]). Of course,this can lead to some quantitative modifications of the results but their main qualitative features as a possibility for new narrow in-gap quasiparticle bands and related sharp resonant peaks in transport coefficients should be still present

The experimental verifications of these predictions would be of evident interest, since they can open perspectives for important practical applications, e.g., in narrow-band microwave devices or advanced low-temperature sensors. However this would impose rather hard requirements on the quality and composition of the necessary samples; they should be extremely pure aside the extremely low (by common standards) and well-controlled contents of specially chosen and uniformly distributed impurity centers within the SC iron-arsenic planes of a ferropnictide compound. This situation can be compared to the requirements on doped semiconductor devices and hopefully should not be a real problem for modern lab technologies. 
Chapter 6

\section{Electric bias control on impurity effects in bigraphene}




\title{
Electric bias control of impurity effects in bilayer graphene
}

\author{
Y. G. Pogorelov, ${ }^{1}$ M. C. Santos, ${ }^{2}$ and V. M. Loktev ${ }^{3,4}$ \\ ${ }^{1}$ IFIMUP-IN, Departamento de Física, Universidade do Porto, Rua do Campo Alegre 687, Porto 4169-007, Portugal \\ ${ }^{2}$ Departamento de Física, Universidade de Coimbra, R. Larga, Coimbra 3004-535, Portugal \\ ${ }^{3}$ Bogolyubov Institute for Theoretical Physics, NAN of Ukraine, 14b Metrologichna Str., Kiev 03680, Ukraine \\ ${ }^{4}$ National Technical University of Ukraine KPI, Pr. Peremogy 37, Kiev 03056, Ukraine \\ (Received 15 October 2014; revised manuscript received 26 May 2015; published 3 August 2015) \\ Formation of localized impurity levels within the band gap in bigraphene under applied electric field and the \\ conditions for their collectivization at finite impurity concentrations are considered. It is shown that a qualitative \\ restructuring of the quasiparticle spectrum within the initial band gap and subsequent metal-insulator phase \\ transitions are possible for such disordered systems, being effectively controlled by variation of the electric field \\ bias. Since these effects can be expected at low enough impurity concentrations and accessible applied voltages, \\ they can be promising for practical applications in nanoelectronics devices.
}

DOI: 10.1103/PhysRevB.92.075401

PACS number(s): 74.70.Xa, 74.62.-c, 74.62.Dh, 74.62.En

\section{INTRODUCTION}

Between various derivatives from the basic graphene system [1-3], special interest is attributed to its bilayer combination [4]. This interest is mainly due to the important possibility of realizing here a semiconductor with a controllable band gap through the application of an electric field normal to the layers [5-8]. It should be noted that a similar crystalline structure of two planes with hexagonal lattices is now recognized for a whole family of materials, either really fabricated or theoretically predicted. Besides the two known modifications of bilayer graphene itself, the Bernal (or A-B) structure [9] and its alternative, the A-A structure [10], there exist also the bilayers of silicene, the silicon analog to graphene [11], the bilayers of boron nitride [12] or its bilayered combination with graphene [13], the bilayered chalcogenides of transition metals (pure or alloyed) [14], etc. However, the most reliable structure for external tuning and rather simple for theoretical study is seen in the Bernal-stacked bilayer graphene, hence chosen here as the basic host system for studying impurity effects. Having introduced impurities in such a system, such as dopants in common semiconductor systems $[15,16]$, there is a possibility for localized impurity levels to appear within the host spectrum gap [17,18]. Next, it is known that, at high enough impurity concentration, an intensive interaction between the localized impurity states related to these levels can take place. This can essentially modify the band spectrum near the gap edge $[19,20]$, giving rise to specific narrow energy ranges of bandlike states near impurity levels (called impurity bands) and even producing a phase transition from insulating to metallic states [21], with important practical applications. An attempt to treat such impurity bands in doped bilayer graphene was done by Nilsson and Castro Neto [17]; however, it missed the crucial issue of whether the respective states in a disordered crystal are really bandlike (extended) or localized and where the separation points between these two kinds in the energy spectrum (the mobility edges [21]) are located. A consistent study of these questions is one of our main purposes here. Having it resolved, the resulting possibility of continuous control on band gap and of controllable phase transitions can make the in-gap impurity states in bilayer graphene quite a flexible tool with regard to electronic properties. A similar situation was recognized long ago in some magnetic crystals with impurities at the magnon spectra, and so the observable properties can be controlled by an applied magnetic field [22]. Such a possibility for fermionic systems could open interesting possibilities for future nanoelectronics.

The paper is organized as follows. In Sec. II, the second quantization Hamiltonian is defined for a biased Bernalstacked graphene bilayer (with no impurities) and the related matrix representation for Green's functions (GFs) is built, giving rise to its four-subband electronic spectrum. Section III introduces the model impurity perturbation and analyzes formation of impurity levels and their possible development into impurity bands, based on specific self-energy matrices for the GF matrices. Such impurity bands are considered in more detail in Sec. IV, including the estimates for mobility edges between the bandlike and localized states. Then the possibility for metal-insulator phase transitions in doped bilayer graphene under electric field bias (at fixed impurity concentration) and the resulting transport effects are analyzed in Sec. V. The final Sec. VI presents the main conclusions and suggestions for practical applications of the described impurity effects.

\section{BILAYER GRAPHENE UNDER APPLIED FIELD}

As is well known, the relevant electronic dynamics of a graphene sheet are generated by the carbon $s p^{3}$ orbitals (whose energy level can be chosen as the energy reference) in the simplest approximation of single hopping parameter $t$ between nearest-neighbor carbons from different sublattices at distance $a$ in the honeycomb lattice [2]. The bilayer graphene structure, furthermore, involves the interlayer hopping $t_{z}$ by vertical links between nearest neighbors from different sublattices (for Bernal stacking) shown in Fig. 1. With an account taken of an electric bias $V=e E d$ between the layers (with the electron charge $e$, the applied electric field $E$, and the interlayer spacing $d$ ), this defines the tight-binding (Fourier-transformed) Hamiltonian $4 \times 4$ matrix [6]:

$$
\hat{H}_{\mathbf{k}}=\left(\begin{array}{cccc}
V / 2 & \gamma_{\mathbf{k}} & 0 & t_{z} \\
\gamma_{\mathbf{k}}^{*} & V / 2 & 0 & 0 \\
0 & 0 & -V / 2 & \gamma_{\mathbf{k}} \\
t_{z} & 0 & \gamma_{\mathbf{k}}^{*} & -V / 2
\end{array}\right) .
$$




\title{
Electric bias control on impurity effects in bigraphene
}

\author{
Y.G. Pogorelov, ${ }^{1}$ M.C. Santos ${ }^{2}$ V.M. Loktev ${ }^{3,4}$ \\ ${ }^{1}$ IFIMUP-IN, Departamento de Física, Universidade do Porto, Rua do \\ Campo Alegre 687, Porto 4169-007, Portugal, \\ ${ }^{2}$ Departamento de Física, Universidade de Coimbra, R. Larga, Coimbra \\ 3004-535, Portugal, \\ ${ }^{3}$ Bogolyubov Institute for Theoretical Physics, NAN of Ukraine, 14b \\ Metrologichna str., Kiev 03680, Ukraine \\ ${ }^{4}$ National Technical University of Ukraine "KPI", Pr. Peremogy 37, Kiev \\ 03056, Ukraine
}

Formation of localized impurity levels within the band gap in bigraphene under applied electric field and the conditions for their collectivization at finite impurity concentrations are considered. It is shown that a qualitative restructuring of the quasiparticle spectrum within the initial band gap and subsequent metal-insulator phase transitions are possible for such disordered systems, being effectively controlled by variation of the electric field bias. Since these effects can be expected at low enough impurity concentrations and accessible applied voltages, they can be promising for practical applications in nanoelectronics devices.

\section{Introduction}

Between various derivatives from the basic graphene system $[7,8,9]$, special interest is attributed to its bilayer combination [10]. This interest is mainly due to the important possibility of realizing here a case of semiconductor with controllable band gap through the application of an electric field $[200,201,202,203]$. It should be noted that a similar crystalline structure of two planes with hexagonal lattices is now recognized for a whole family of materials, either really fabricated or theoretically predicted. Besides the two known modifications of bigraphene itself, the Bernal (or A-B) structure [204] and its alternative, A-A structure [205], there exist also the bilayers of silicene, the silicon analog to graphene [206], the bilayers of boron nitride [207] or its bilayered combination with graphene [208], the bilayered chalcogenides of transition metals (pure or alloyed) [209], etc. However, the most reliable structure for external tuning and rather simple for theoretical study is seen in the Bernal-stacked bilayer graphene, hence chosen here as the basic host system for studying impurity effects. Having introduced impurities in such a system, such as dopants in common semiconductor systems [34, 210], there is a possibility for localized impurity levels to appear within the host spectrum 
gap [211, 212]. Next, it is known that, at high enough impurity concentration, an intensive interaction between the localized impurity states related to these levels can take place. This can essentially modify the band spectrum near the gap edge [165, 213], giving rise to specific narrow energy ranges of bandlike states near impurity levels (called impurity bands) and even producing a phase transition from insulating to metallic state [169], with important practical applications. An attempt to treat such impurity bands in doped bilayer graphene was done by Nilsson and Castro Neto [211]; however, it missed the crucial issue of whether the respective states in a disordered crystal are really bandlike (extended) or localized and where the separation points between these two kinds in the energy spectrum (the mobility edges [169]) are located. A consistent study of these questions is one of our main purposes here. Having it resolved,the resulting possibility of continuous control on band gap and of controllable phase transitions can make the in-gap impurity states in bilayer graphene quite a flexible tool with regard to electronic properties. A similar situation was recognized long ago in some magnetic crystals with impurities at the magnon spectra, and so the observable properties can be controlled by an applied magnetic field [132]. Such a possibility for fermionic systems could open interesting possibilities for future nanoelectronics.

The paper is organized as follows. In Sec. II, the second quantization Hamiltonian is defined for a biased Bernal-stacked graphene bilayer (with no impurities) and the related matrix representation for Green's functions (GFs) is built, giving rise to its four-subband electronic spectrum. SectionIII introduces the model impurity perturbation and analyzes formation of impurity levels and their possible development into impurity bands, based on specific self-energy matrices forthe GF matrices. Such impurity bands are considered in more detail in Sec.IV, including the estimates for mobility edges between the bandlike and localized states. Then the possibility for metal-insulator phase transitions in doped bilayer graphene under electric field bias (at fixed impurity concentration) and the resulting transport effects are analyzed in Sec.V. The final Sec.VI presents the main conclusions and suggestions for practical applications of the described impurity effects. 


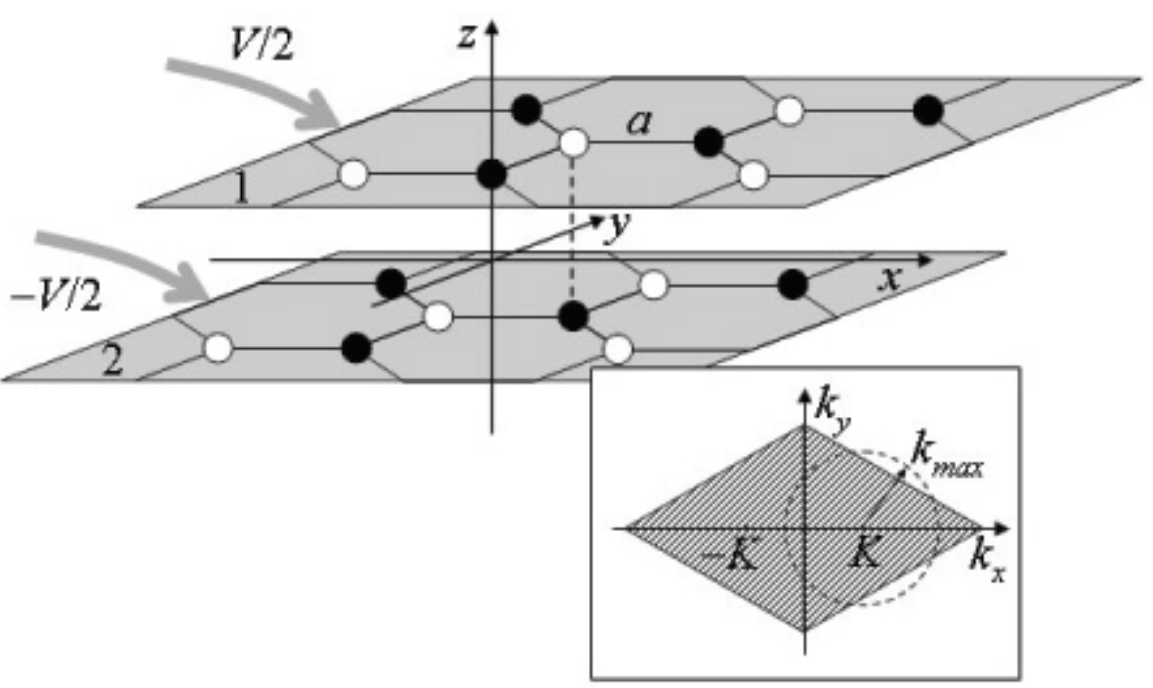

Figure 6.1: Schematic of Bernal-stacked bigraphene under applied electric bias $V$. The Aand B-type sites in each plane are indicated by black and white circles respectively, the solid and dashed lines indicate the in-plane $t$ and interplane $t_{z}$ links. Inset: the Brillouin zone in k-plane with two Dirac points $\pm \mathbf{K}$ and an equivalent circle of radius $k_{\max }=\sqrt{K / a}$.

\section{Bigraphene under Applied Field}

As is well known, the relevant electronic dynamics of a graphene sheet are generated by the carbon $s p^{3}$ orbitals (whose energy level can be chosen as the energy reference) in the simplest approximation of single hopping parameter $t$ between nearest neighbor carbons from different sublattices at distance $a$ in the honeycomb lattice [8]. The bigraphene structure, furthermore, involves the interlayer hopping $t_{z}$ by vertical links between nearest neighbors from different sublattices (for Bernal stacking) shown in Fig. 6.1. With an account taken of an electric bias $V=e E d$ between the layers (with the electron charge $e$, the applied electric field $E$, and the interlayer spacing $d$ ), this defines the tightbinding (Fourier transformed) Hamiltonian $4 \times 4$ matrix [214]:

$$
\hat{H}_{\mathbf{k}}=\left(\begin{array}{cccc}
V / 2 & \gamma_{\mathbf{k}} & 0 & t_{z} \\
\gamma_{\mathbf{k}}^{*} & V / 2 & 0 & 0 \\
0 & 0 & -V / 2 & \gamma_{\mathbf{k}} \\
t_{z} & 0 & \gamma_{\mathbf{k}}^{*} & -V / 2
\end{array}\right)
$$


Here the wave vector $\mathbf{k}$ lies in the first Brillouin zone (see inset in Fig. 6.1) and the in-plane dispersion follows from the sums $\gamma_{\mathbf{k}}=t \sum_{\delta} \mathrm{e}^{i \mathbf{k} \cdot \boldsymbol{\delta}}$ over nearest neighbor vectors $\boldsymbol{\delta}$ of the honeycomb lattice. It suitably approximates as $\gamma_{\mathbf{k}} \approx$ $\xi_{\mathbf{k}} \mathrm{e}^{i \varphi_{\mathbf{k}}}$, with $\xi_{\mathbf{k}}=\hbar v_{\mathrm{F}}|\mathbf{k}-\mathbf{K}|$ near the Dirac points $\mathbf{K}= \pm(4 \pi / 3 \sqrt{3} a, 0)$, where the Fermi velocity $v_{\mathrm{F}}=3 t a / 2 \hbar$, and $\varphi_{\mathbf{k}}=\arctan k_{y} /\left(k_{x}-K_{x}\right)$. The relevant range of $|\mathbf{k}-\mathbf{K}| \sim K t_{z} / W$ is really narrow, since $t_{z}$ is weak besides the total bandwidth $W$ (see below). Then the second-quantization Hamiltonian (in absence of impurity perturbation),

$$
H_{0}=\sum_{\mathbf{k}} \psi_{\mathbf{k}}^{\dagger} \hat{H}_{\mathbf{k}} \psi_{\mathbf{k}}
$$

where the spinors $\psi_{\mathbf{k}}^{\dagger}=\left(a_{1 \mathbf{k}}^{\dagger}, b_{1 \mathbf{k}}^{\dagger}, a_{2 \mathbf{k}}^{\dagger}, b_{2 \mathbf{k}}^{\dagger}\right)$ made of Fourier transformed second quantization operators $a_{j \mathbf{k}}=N^{-1} \sum_{\mathbf{n}} a_{j \mathbf{n}} \mathrm{e}^{i \mathbf{k} \cdot \mathbf{n}}$ and $b_{j \mathbf{k}}=N^{-1} \sum_{\mathbf{n}} b_{j \mathbf{n}} \mathrm{e}^{i \mathbf{k} \cdot \mathbf{n}}$, where the on-site operators $a_{j \mathbf{n}}$ and $b_{j \mathbf{n}}$ relate for A- and B-type sites from $n$th unit cell in $j(=1,2)$ th layer, and $N$ is the number of cells in a layer. Generally, the energy spectrum is defined by the matrix of Fourier-transformed two-time GFs $[166,215] \hat{G}_{\mathbf{k}}=\left\langle\left\langle\psi_{\mathbf{k}} \mid \psi_{\mathbf{k}}^{\dagger}\right\rangle\right\rangle$ as solutions of the dispersion equation:

$$
\operatorname{Re} \operatorname{det} \hat{G}_{\mathbf{k}}^{-1}=0
$$

Thus for the nonperturbed system by Eq. (6.2), the GF matrix reads $\hat{G}_{\mathrm{k}}^{(0)}=$ $\left(\varepsilon-\hat{H}_{\mathbf{k}}\right)^{-1}$ and, after diagonalization of $\hat{H}_{\mathbf{k}}$ in spinor indices, its dispersion near the Dirac points is suitably expressed through the radial variable $\xi_{\mathrm{k}} \equiv \xi$. It includes two positive energy subbands [214]:

$$
\varepsilon_{\nu}(\xi)=\sqrt{\frac{t_{z}^{2}}{2}+\frac{V^{2}}{4}+\xi^{2}-(-1)^{\nu} \sqrt{\frac{t_{z}^{4}}{4}+\xi^{2}\left(t_{z}^{2}+V^{2}\right)}},
$$

the "external" $(\nu=1)$ and "internal" $(\nu=2)$ ones, and their negative energy counterparts, as shown in Fig. 6.2a. The most relevant feature of this spectrum is the bias-controlled energy gap between the extrema $\pm \varepsilon_{g}=$ $\pm V /\left[2 \sqrt{1+\left(V / t_{z}\right)^{2}}\right]$ of two internal subbands, attained along a circle around each Dirac point (the so-called Mexican hat) whose radius in the $\xi$-variable is $\xi_{0}=\sqrt{\varepsilon_{g}^{2}+V^{2} / 4}$.

The GF matrix generates physical characteristics of this system as, for instance, the density of states (DOS) by electronic quasiparticles, 


$$
\rho(\varepsilon)=\frac{1}{\pi} \operatorname{Im} \operatorname{Tr} \hat{G}(\varepsilon),
$$

where $\hat{G}(\varepsilon)=(2 N)^{-1} \sum_{\mathbf{k}} \hat{G}_{\mathbf{k}}(\varepsilon)$ is the local GF matrix, and its imaginary part for exact band spectrum results usually from an infinitesimal imaginary shift of energy argument, $\varepsilon-i 0$ [166]. In what follows, the sum in $\mathbf{k}$ over triangular halves of the Brillouin zone is approximated by the $\xi$-integration,

$$
\frac{1}{2 N} \sum_{\mathbf{k}} f_{\mathbf{k}}(\varepsilon) \approx \frac{2}{W^{2}} \int_{0}^{W} f(\xi, \varepsilon) \xi d \xi
$$

over two equivalent circles around the Dirac points (inset in Fig. 6.1) of the $\xi$-radius $W=\hbar v_{\mathrm{F}} k_{\max }$ (where $k_{\max }=\sqrt{K / a}$, see inset in Fig. 6.1). This approximation is well justified at low energies, $|\varepsilon| \ll W$, compared to the effective bandwidth $W$. For the pure bigraphene system by Eq. (6.1), the result for Eq. (6.5) is generated by the explicit diagonal elements of nonperturbed local GF matrix [211]:

$$
\begin{aligned}
G_{11}^{(0)} & \approx 2 \frac{\varepsilon-\varepsilon_{2}}{W^{2}}\left[\frac{\varepsilon \varepsilon_{2}}{\delta^{2}}\left(\pi-\arctan \frac{\delta^{2}}{\varepsilon^{2}+\varepsilon_{2}^{2}}\right)+\ln \frac{\gamma}{W}\right], \\
G_{22}^{(0)} & =G_{11}^{(0)}(\varepsilon)-t_{z}^{2} \frac{\varepsilon+\varepsilon_{2}}{W^{2} \delta^{2}}\left(\pi-\arctan \frac{\delta^{2}}{\varepsilon^{2}+\varepsilon_{2}^{2}}\right),
\end{aligned}
$$

where

$$
\begin{aligned}
& \delta^{2}(\varepsilon)=\sqrt{\left(t_{z}^{2}+V^{2}\right)\left(\varepsilon_{g}^{2}-\varepsilon^{2}\right)}, \\
& \gamma^{2}(\varepsilon)=\sqrt{\left(\varepsilon^{2}-\varepsilon_{1}^{2}\right)\left(\varepsilon^{2}-\varepsilon_{2}^{2}\right)} .
\end{aligned}
$$

These elements reveal the inverse square root divergences at the gap edges $\pm \varepsilon_{g}$ (of $\operatorname{Im} G$ beyond the gap and of $\operatorname{Re} G$ within the gap); also note the finite steps of $\operatorname{Im} G$ at the limiting energies $\varepsilon_{1,2} \equiv \varepsilon_{1,2}(0)$ of the two subbands. The resting diagonal elements are simply $G_{33}^{(0)}(\varepsilon)=-G_{22}^{(0)}(-\varepsilon)$ and $G_{44}^{(0)}(\varepsilon)=-G_{11}^{(0)}(-\varepsilon)$, so that finally DOS is a function of $\varepsilon^{2}$, as shown in Fig. $6.2 \mathrm{~b}$, in agreement with the known previous calculations [203]. It presents BCS-like divergences near $\varepsilon_{g}^{2}$, finite steps at $\varepsilon_{1,2}^{2}$, and coincides with the linear DOS for monolayer graphene $[8,9]$ beyond $\varepsilon_{1}^{2}$, due to joint (non-linear) contributions from both subbands. 


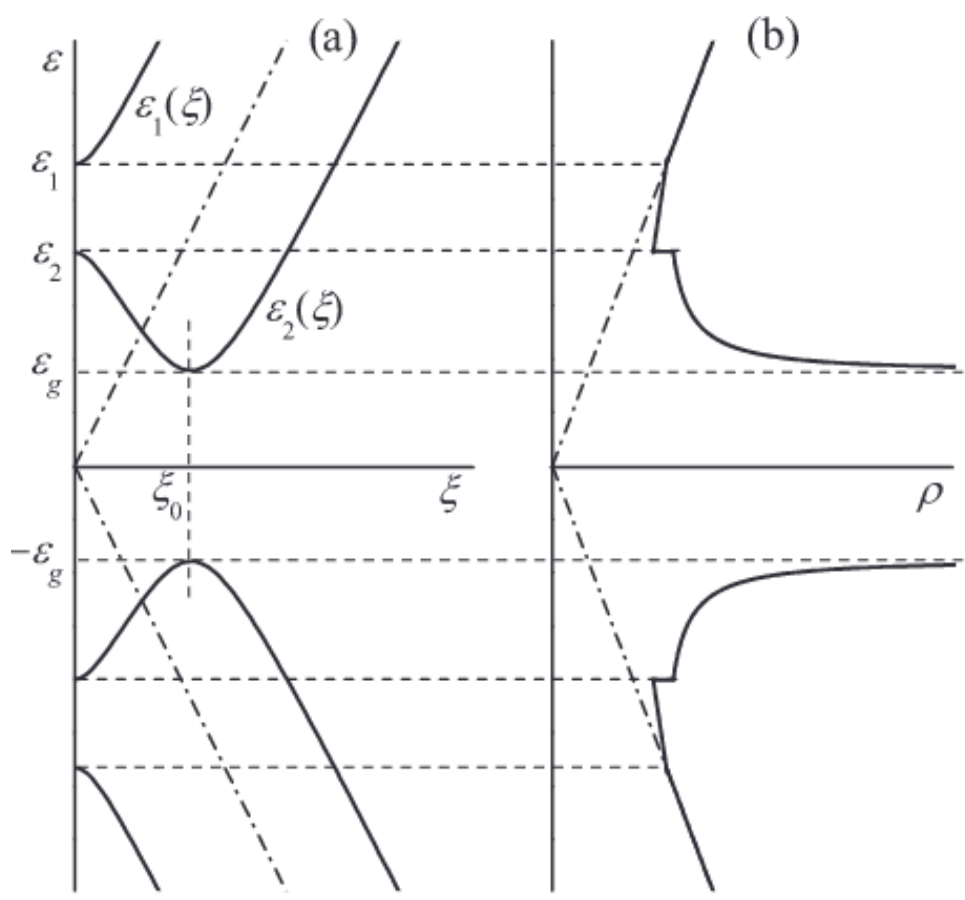

Figure 6.2: (a) Dispersion laws for the bilayer in Fig. 6.1 vs the radial variable $\xi$ near a Dirac point, given by Eq. (??) at the choice of $V=2 t_{z}$; the dash-dotted line indicate the Dirac dispersion for monolayered graphene. (b) DOS for this choice; the dash-dotted line marks the linear DOS for monolayered graphene. 
Within the gap, only real parts of $G_{j j}(\varepsilon)$ are nonzero, and their divergences near the gap edges are crucial for appearance, under the effect of localized impurity perturbations, of in-gap localized levels and related collective states, which is the main focus for the analysis below.

\section{Impurity Levels and Impurity Subbands}

As was recognized from experimental studies on graphene systems [216], they can contain a variety of defects, ranging from topological ones (vacancies, dislocations, edges, boundaries, etc.) to impurity adatoms (or some functional groups) near one of the planes and in-plane substitutes or interstitials. This provides a doping of charge carriers (of both signs) into these systems, as well as scattering of carriers on impurity potentials and possibly formation of localized (or resonance) impurity states on such potentials. The latter must be characterized by some model parameters within the common tight-binding approximation, and the simplest case is the Lifshitz model, involving only the on-site perturbation potential $U$, identical for all impurity sites randomly distributed among the lattice sites [92]. This model was already used in the literature on impurity problems in graphene systems, with $U$ values ranging from the Born regime, $|U| \ll W[211,217,218]$, to the unitary limit, $U \gg W$ $[218,219]$. For the case of substitutional impurities in graphene, this value can be roughly estimated by the differences between the first ionization potential of $11.26 \mathrm{~V}$ for $\mathrm{C}$ and those for its neighbors in the periodic table: $8.3 \mathrm{~V}$ for $\mathrm{B}$ and $14.53 \mathrm{~V}$ for $\mathrm{N}$. Then, for the commonly adopted graphene bandwidth of $W \approx 7 \mathrm{eV}$, the choice of $|U| / W \sim 1 / 2$ looks to be plausible. Also, for diluted impurities at separations much greater than the screening radius [217], the Lifshitz model looks more adequate than the alternative choice of the Anderson model [113], with random perturbations at each lattice site in Ref. [220]. Another alternative is the Anderson hybride (or $s$ - $d$ ) model [221] with two parameters, the impurity binding energy and its coupling to the host excitations. Its use for the so-called deep impurity levels in semiconductors is known to result in formation of the above-mentioned impurity bands and related phase transitions [165]. However, such a perturbation model, when introduced into the framework of a four-component host spectrum of Sec. II, could make the treatment of interactions between impurities and of impurity band coherence technically unfeasible. This determines our choice for the Lifshitz model (though known to provide less freedom for impurity band formation than the $s$ - $d$ model). Due to similar reasons, we do not consider the long-range impurity potentials [222, 223] 
Let us build the perturbation Hamiltonian by Lifshitz impurities on certain impurity sites. In accordance with the composition of $\psi$-spinors, the A and B sites from first plane can be referred to the types $j=1,2$, respectively and those from second plane to $j=3,4$, then $\mathbf{p}_{j}$ denote the defect sites of $j$ th type with relative concentrations $c_{j}=\sum_{\mathbf{p}_{j}} N^{-1}$ such that the total impurity concentration $\sum_{j} c_{j}=c \ll 1$. Then the sought Hamiltonian in terms of local Fermi operators reads

$$
\begin{aligned}
H_{1} & =U\left(\sum_{\mathbf{p}_{1}} a_{1 \mathbf{p}_{1}}^{\dagger} a_{1 \mathbf{p}_{1}}+\sum_{\mathbf{p}_{2}} b_{1 \mathbf{p}_{2}}^{\dagger} b_{1 \mathbf{p}_{2}}\right. \\
& \left.+\sum_{\mathbf{p}_{3}} a_{2 \mathbf{p}_{3}}^{\dagger} a_{2 \mathbf{p}_{3}}+\sum_{\mathbf{p}_{4}} b_{2 \mathbf{p}_{4}}^{\dagger} b_{2 \mathbf{p}_{4}}\right)
\end{aligned}
$$

or, in terms of $\psi$-spinors by Eq. 6.2 , it takes the form of scattering operator,

$$
H_{1}=\frac{1}{N} \sum_{j, \mathbf{p}_{j}} \sum_{\mathbf{k}, \mathbf{k}^{\prime}} \mathrm{e}^{i\left(\mathbf{k}^{\prime}-\mathbf{k}\right) \cdot \mathbf{p}_{j}} \psi_{\mathbf{k}}^{\dagger} \hat{U}_{j} \psi_{\mathbf{k}^{\prime}}
$$

where the diagonal matrix $\hat{U}_{j}$ has a single non-zero element $U$ at the $j j$ site. Considering now the Hamiltonian in presence of impurities $H_{0}+H_{1}$ and following a similar routine to Refs. [23, 224], we arrive at solutions for the GF matrix in two specific forms adequate for two alternative types of excitation states in a disordered system [92, 169]: the bandlike (extended) states and localized states. Thus, the first of these types is better described by the so-called fully renormalized representation (FR) of GF [132],

$$
\hat{G}_{\mathbf{k}}=\left[\left(\left(\hat{G}_{\mathbf{k}}^{(0)}\right)^{-1}-\hat{\Sigma}_{\mathbf{k}}\right]^{-1},\right.
$$

providing the roots of the dispersion equation [Eq. (6.3)], classified along the quasimomentum $\mathbf{k}$. Here the self-energy matrix is additive in different types of impurity centers: $\hat{\Sigma}_{\mathbf{k}}=\sum_{j} \hat{\Sigma}_{j, \mathbf{k}}$, with the partial matrices given by the related FR group expansions (GEs) in complexes of impurity centers (of the same $j$ type, involved in multiple scattering processes): 


$$
\begin{aligned}
\hat{\Sigma}_{j, \mathbf{k}} & =c_{j} \hat{T}_{j}\left[1+c_{j} \sum_{\mathbf{n} \neq 0}\left(\mathrm{e}^{-i \mathbf{k} \cdot \mathbf{n}} \hat{A}_{j, \mathbf{n}}+\hat{A}_{j, \mathbf{n}} \hat{A}_{j,-\mathbf{n}}\right)\right. \\
& \left.\times\left(1-\hat{A}_{j, \mathbf{n}} \hat{A}_{j,-\mathbf{n}}\right)^{-1}+\ldots\right] .
\end{aligned}
$$

Each T matrix $\hat{T}_{j}=\hat{U}_{j}\left(1-\hat{G} \hat{U}_{j}\right)^{-1}$ describes all the scatterings on a single impurity center of $j$ th type, and the next-to-unity term in right-hand side of Eq. (6.10) accounts for scatterings on pairs of $j$-impurities through the matrices $\hat{A}_{j, \mathbf{n}}=\hat{T}_{j}(2 N)^{-1} \sum_{\mathbf{k}^{\prime} \neq \mathbf{k}} \hat{G}_{\mathbf{k}^{\prime}}$ e $^{i \mathbf{k}^{\prime} \cdot \mathbf{n}}$ of indirect interaction (via bandlike excitations) in such pairs at separation $\mathbf{n}$. Notice the excluded quasimomentum $\mathbf{k}$ (for given $\hat{\Sigma}_{\mathbf{k}}$ ) in this sum; also the FR GE excludes coinciding quasimomenta in all the multiple sums for products of interaction matrices [132]. The omitted terms in Eq. (6.10) relate to all scattering processes in groups of three and more impurities, and their general structure can be found in similarity with the known group integrals from the Ursell-Mayer classical theory of nonideal gases.

Otherwise, for the range of localized states, the nonrenormalized representation (NR),

$$
\hat{G}_{\mathbf{k}}=\hat{G}_{\mathbf{k}}^{(0)}-\hat{G}_{\mathbf{k}}^{(0)} \hat{\Sigma} \hat{G}_{\mathbf{k}}^{(0)},
$$

defining rather DOS from Eq. (6.5) than dispersion from Eq. (6.3), is more adequate. Here the respective NR self-energy matrix $\hat{\Sigma}=\sum_{j} \hat{\Sigma}_{j}$ has a similar structure to the FR structure one by Eq. (6.10) but with the NR matrices $\hat{T}_{j}^{(0)}=\hat{U}_{j}\left(1-\hat{G}^{(0)} \hat{U}_{j}\right)^{-1}, \hat{G}^{(0)}=(2 N)^{-1} \sum_{\mathbf{k}} \hat{G}_{\mathbf{k}}^{(0)}$, and with no restrictions in all the quasimomentum sums for the products of NR interaction matrices $\hat{A}_{j, \mathbf{n}}^{(0)}=\hat{T}_{j}^{(0)}(2 N)^{-1} \sum_{\mathbf{k}} \hat{G}_{\mathbf{k}}^{(0)} \mathrm{e}^{i \mathbf{k} \cdot \mathbf{n}}$ (that are only present in their even combinations $\left.\hat{A}_{j, \mathbf{n}}^{(0)} \hat{A}_{j,-\mathbf{n}}^{(0)}\right)$.

The best-known effect of local perturbations consists in emergence of localized energy levels within the band gap, and those were already indicated for impurities in bilayer graphene [211, 212]. As known from general theory [92, 132], such levels are most pronounced at sufficiently low concentration of impurities (so that their indirect interactions can be neglected) when they are given by the poles of $T$ matrices. In the present case, the matrices $\hat{T}_{j}^{(0)}$ give rise to four different local levels $\varepsilon^{(j)}$ within the band gap, and their locations depend on the magnitude and sign of perturbation parameter $U$ (like the known situations in 


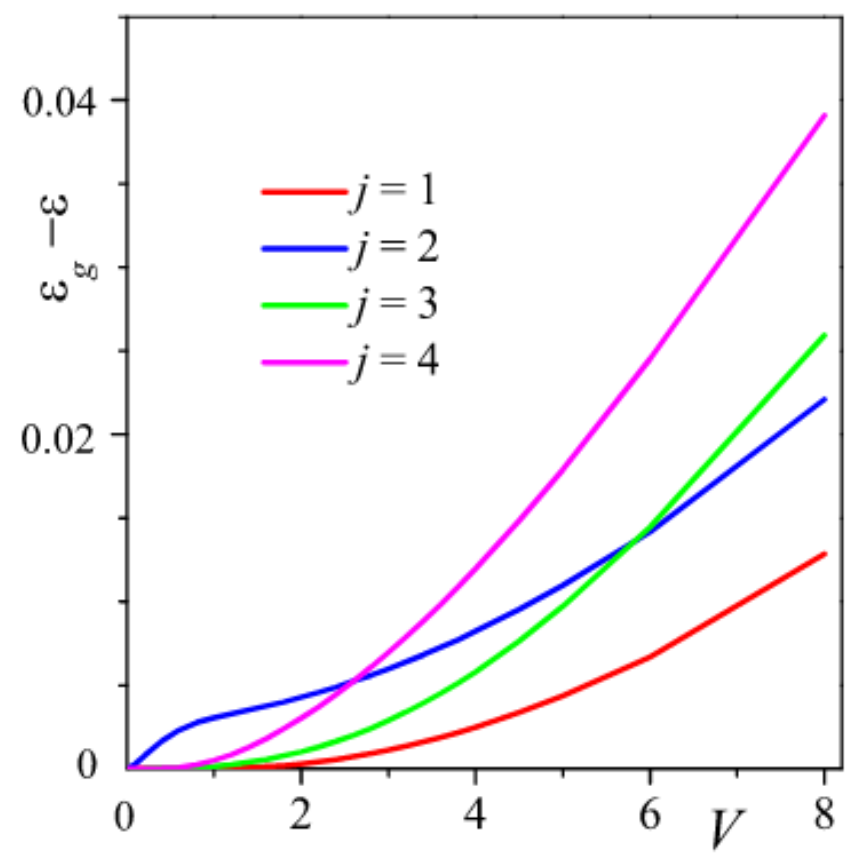

Figure 6.3: Separations of the in-gap impurity levels $\varepsilon^{(j)}$ from the gap edge as functions of the applied bias $V$ (all in units of $t_{z}$ ) for the choice of impurity perturbation parameter $U=-W / 2$. Note the different behaviors of $\varepsilon^{(1,2)}$ and $\varepsilon^{(3,4)}$ pairs and the interchange of the deepest level from $\varepsilon^{(2)}$ to $\varepsilon^{(4)}$ at the bias value $V_{c r} \approx 2.6 t_{z}$ (see also the text). 
common doped semiconductors [34, 210]) but yet on the applied field $V$ (as a specifics of doped bilayer graphene). The positions of four impurity levels $\varepsilon^{(j)}$ by each type of impurity center are the roots of related Lifshitz equations,

$$
U G_{j j}^{(0)}\left(\varepsilon^{(j)}\right)=1,
$$

so that choosing for definiteness $U=-W / 2$ and using Eq. (6.6) provides their dependence on the applied bias $V$ as shown in Fig. 6.3 (for their relative separations from the gap edge). It is seen that generally they stay rather shallow at growing $V$, but with a notable difference between the levels $\varepsilon^{(1,2)}$ (by impurities in the positive biased layer) and $\varepsilon^{(3,4)}$ (by those in the negative biased one). In particular, a specific interchange of the deepest levels occurs in this course, from $\varepsilon^{(2)}$ to $\varepsilon^{(4)}$, at $V_{c r} \approx 2.6 t_{z}$ for the given $U$. This feature was not indicated in the former analysis of the same model in Ref. [211], where only $\varepsilon^{(2)}$ was considered as the deepest level. However, for the commonly used value of $t_{z} \approx 0.35 \mathrm{eV}$, this interchange bias would amount to $V_{c r} \approx 0.91 \mathrm{eV}$, well above the experimentally realized (to the moment) $V$ values of up to $\approx 0.36$ $\mathrm{eV}[200,201]$. Thus, the much stronger separation of the $\varepsilon^{(2)}$ level at lower bias voltages could be of significant practical importance.

Also, we note that while the impurity levels generally become deeper at greater $U$ values, the indicated interchange bias decreases in this course: from $V_{c r} \approx$ $3.53 t_{z}$ at $U=-W / 4$ to $V_{c r} \approx 1.88 t_{z}$ at $U=-W$.

The well-known property of localized states by shallow energy levels is their long effective radius [132], also indicated for impurities in biased bilayer graphene [211], defining intensive interactions between them already at their very low concentrations. Such interactions were shown to allow, at certain conditions, collectivization of impurity states to form specific bandlike states within narrow energy bands (called impurity bands) around the initial localized levels [165]. As will be seen below, this effect is possible as well in the present case of multiple localized levels, where the most essential specifics is their joint participation in forming the lowest impurity subband of much stronger dispersion than in higher-lying subbands (if those are permitted).

Formally, in similarity to the nonperturbed case, the band spectrum for the disordered system can be evaluated from the dispersion equation, Eq. (6.3), with the FR GF matrix by Eqs. (6.9) and (??). Of course, if treated rigorously, it presents a tremendous problem of developing infinite sequence of renormalization procedures in all possible terms of the corresponding GE, and there is no reasonable hope for its exact solution. On the other hand, validity 
of the relatively simpler NR, Eq. (6.11), is only limited to the energy ranges of localized states.

One could try to use the coherent potential approximation (CPA) [225], a useful tool, e.g., in the theory of disordered alloys, where the full self-energy is presented in a self-consistent T-matrix form. It reduces the impurity effect at each given energy to a certain spatially uniform potential adjusted to make the average scattering zero. This is done through a stable iterative procedure and readily provides a definite band spectrum. However, this approach treats the disordered system as if keeping unbroken translation symmetry and so leads to a purely extended spectrum, unlike its real composition of bandlike and localized ranges [169]. Therefore the CPA results can be only justified within the bandlike ranges, far enough from their edges [194], accordingly to the known Ioffe-Regel-Mott (IRM) criterion of long enough mean free path compared to the wavelength $[169,53]$. Moreover, for the disorder due to diluted impurities, CPA applies only to the less perturbed interiors of the broad initial bands but not to the narrow impurity bands between close mobility edges. This can be verified by comparing its results to those by more consistent theories or to experimental data (when available).The CPA versions were also suggested for impurity effects both in monolayer graphene [226] and bilayer graphene [211], producing in the latter case some band features within the initial band gap. But our analysis below, starting from the same structure of impurity levels as in Ref. [211], results in a quite different picture of impurity bands and we justify it based on the IRM criteria relevant for this case.

The practical approach is done through partial renormalizations of the full self-energy in Eq. (6.10), first substituting there the NR T matrix and interaction matrices and then subsequently introducing such approximate selfenergies into the next generations of GF and interaction matrices. In this course, convergence of the obtained GEs is checked in order not to extend the renormalizations to irrelevant GE terms. Namely, it is reasonable to define the $l$ th generation GF matrix $\hat{G}_{\mathbf{k}}^{(l)}$ by an analog to Eq. (6.9) with the respective self-energy $\hat{\Sigma}_{\mathbf{k}}^{(l)}$ by an analog to Eq. (6.10) but containing the matrices $\hat{T}^{(l-1)}$ and $\hat{A}_{\mathbf{n}}^{(l-1)}$ built from the preceding generation $\hat{G}_{\mathrm{k}}^{(l-1)}$ matrices. This algorithm leads to the true FR at $l \rightarrow \infty$. However, even its first non-trivial $l=1$ approximation can be reasonable for the bandlike energy ranges where the true FR GE converges.

Then, in the first step of this routine, the formal solutions of Eq. (6.10) with the self-energies in the NR T-matrix approximation, $\hat{\Sigma}_{j, \mathbf{k}} \approx c_{j} \hat{T}_{j}^{(0)}$, display four narrow subbands near four impurity levels $\varepsilon^{(j)}$, besides the four broad 


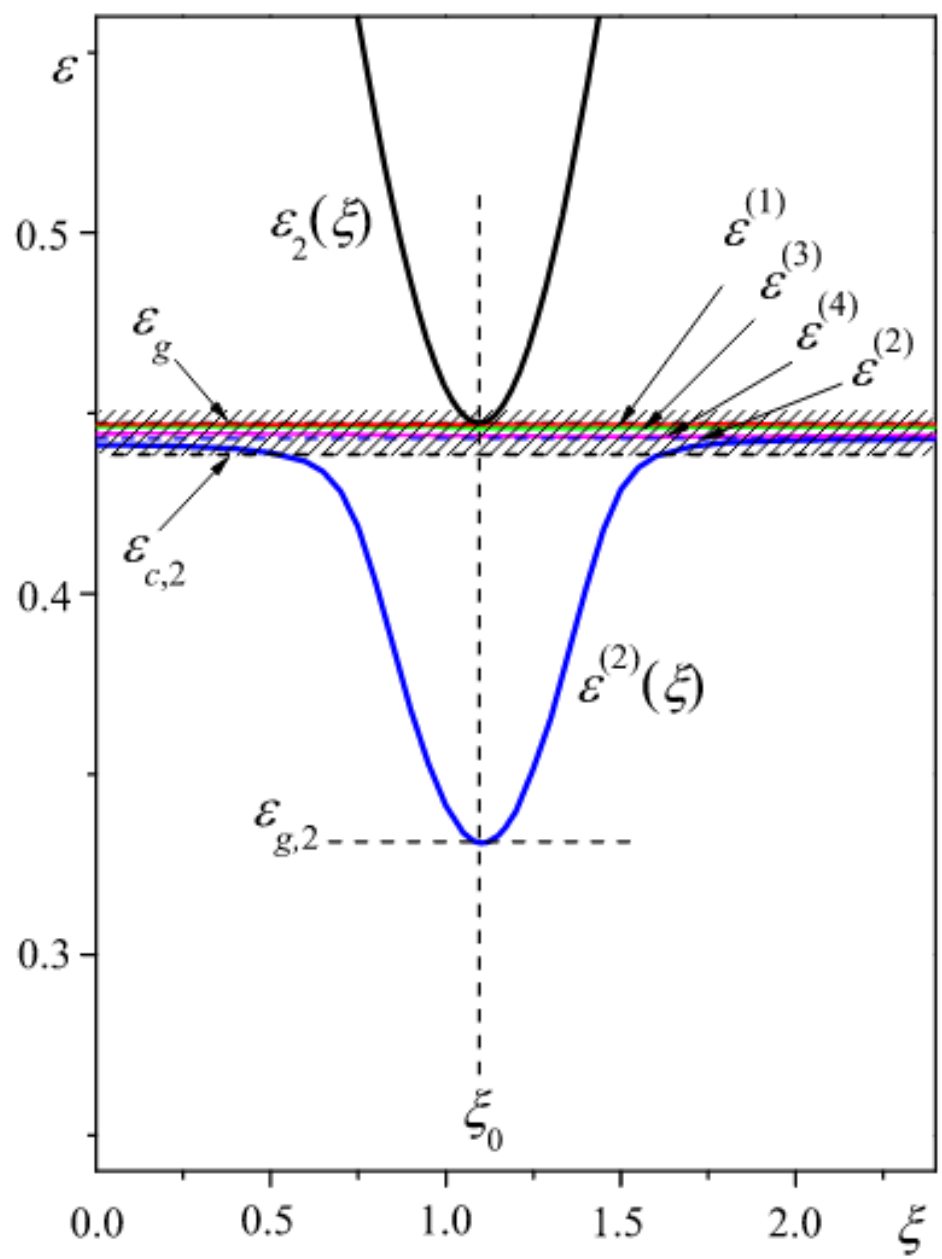

Figure 6.4: Formation of impurity subbands near the impurity levels by the solutions of Eq. (6.3) in the first step of renormalization (see text) for the case of Fig. 6.3 at $V=2 t_{z}$ and $c=0.01$ (with the variables $\varepsilon$ and $\xi$ presented in $t_{z}$ units). Only the most dispersive $\varepsilon^{(2)}(\xi)$ subband extends well beyond the shadowed vicinity of the $\varepsilon^{(2)}$ level, which delimits the range of localized states down to the respective mobility edge $\varepsilon_{c, 2}$. Together with the localized states around $\varepsilon^{(4,3,1)}$, this range continues up to above the gap edge $\varepsilon_{g}$. 
principal bands $\pm \varepsilon_{\nu}(\xi)$ [here only slightly modified compared to Eq. (??)]. An example of such modified spectrum (at a natural choice of equal partial concentrations $c_{j}=c$ and taking the total impurity concentration $4 c=0.01$ ) for the cases of Fig. 6.3 is shown in Fig. 6.4. The lowest impurity subband, conventionally denoted here as $\varepsilon^{(2)}(\xi)$ by its proximity to the lowest $\varepsilon^{(2)}$ level, is seen to strongly dominate in its dispersion over all the resting ones, and the direct analysis of Eq. (6.10) shows that this domination is due to the above-mentioned constructive interplay between all $\varepsilon^{(j)}$.

Note that all the impurity subbands in this approximation produce BCS-like divergences in DOS, as well near the levels $\varepsilon^{(j)}$ as near subbands terminations. However, since quasimomentum is not true quantum number in a disordered system [92], the analysis of its real energy spectrum, especially for the in-gap states, should also take account of the damping $\Gamma_{j}(\xi)$ of each $\varepsilon^{(j)}(\xi)$ state resulting from $\operatorname{Im} \Sigma_{j}$. Hence one can consider these states Bloch-like (or conducting) only if the IRM criterion is fulfilled or the GE, Eq. (6.10), is convergent at related energies. Otherwise they should pertain to the localized type. As will be seen, all the formal DOS singularities fall within the localized energy ranges and so are effectively broadened.

The mentioned criteria also permit to estimate the Mott mobility edges between bandlike and localized ranges. Of course, such mobility edges can be found near the limits of both principal and impurity bands, but our main focus here will be on the most dispersive impurity band, for instance $\varepsilon^{(2)}(\xi)$ in the above example. Finally, a certain special value $V_{\mathrm{A}}$ of bias control (at given impurity concentrations $c_{j}$ and perturbation parameter $U$ ) can be indicated, such that mobility edges from both sides of a conducting impurity band will merge. This collapse of the impurity band will manifest a kind of Anderson transition [113] in a disordered system, realized in a controllable way at $V \rightarrow V_{\mathrm{A}}$.

It should be noted that all these fundamental features of the energy spectrum in a disordered system are lost when the impurity bands are treated withinthe CPA approximation (as, e.g., in Refs. [211] and [226]). 


\section{Conditions for Existence of Impurity Subbands}

As known from studies on many disordered systems where a localized impurity level $\varepsilon_{i m p}$ near an edge $\varepsilon_{g}$ of pure crystal energy band can give rise, at high enough impurity concentration, to a specific impurity band $\varepsilon_{i m p}(\mathbf{k})$ [132], the latter is restricted by the general IRM criterion,

$$
\mathbf{k} \cdot \nabla_{\mathbf{k}} \varepsilon_{i m p}(\mathbf{k}) \gg \Gamma_{i m p}\left(\varepsilon_{i m p}(\mathbf{k})\right)
$$

where the linewidth $\Gamma_{i m p}(\varepsilon)$ of a Bloch-like state with quasimomentum $\mathbf{k}$ and energy $\varepsilon$ is defined as the imaginary part of the corresponding self-energy. For the present multiband system, this criterion should be formulated for each of $\varepsilon^{(j)}(\xi)$ subbands by expanding the general determinant from Eq. (6.3) near a given energy $\varepsilon$ in a complex form, $\operatorname{det} \hat{G}_{\mathbf{k}}^{-1} \approx\left[\varepsilon-\varepsilon^{(j)}(\xi)+i \Gamma_{j}(\varepsilon)\right] \Omega_{j}(\varepsilon)$, to obtain the corresponding linewidth $\Gamma_{j}(\varepsilon)$ [aside a certain factor $\Omega_{j}(\varepsilon)$ of energy-to-cube dimension].

In the adopted Lifshitz model, each partial $\mathrm{T}$ matrix $\hat{T}_{j}$ (regardless of its renormalization) has a single non-zero element at the $j j$ site (alike $\hat{U}_{j}$ itself): $T_{j}=U /\left(1-U G_{j j}\right)$. For the above suggested first step renormalization, we have $\operatorname{Im} T_{j}^{(0)}=0$ for $\varepsilon$ within the band gap. Here the imaginary part of related self-energy function $\Sigma_{j}^{(1)}$ is only due to the GE terms next to unity in Eq. (6.10), dominated by the pair term once GE is convergent. It can be also shown that the most relevant contribution to $\operatorname{Im} \Sigma_{j}(\varepsilon)$ comes from the $j j$ th matrix element of the GE pair term, while those from its other elements (though generally nonzero) are strongly reduced by the quantum interference effects. This contribution:

$$
B_{j}(\varepsilon)=\operatorname{Im} \sum_{n>a} \frac{A_{j, \mathbf{n}}^{(0)} A_{j,-\mathbf{n}}^{(0)}}{1-A_{j, \mathbf{n}}^{(0)} A_{j,-\mathbf{n}}^{(0)}},
$$

can be obtained from the residues at zeros of the denominator, using the explicit spatial behavior of scalar interaction functions (see Appendix for details),

$$
\begin{aligned}
A_{j, \mathbf{n}}^{(0)}(\varepsilon) & =\frac{T_{j}^{(0)}(\varepsilon)}{2 N} \sum_{\mathbf{k}} \mathrm{e}^{i \mathbf{k} \cdot \mathbf{n}}\left(G_{\mathbf{k}}^{(0)}\right)_{j j} \\
& \approx \sqrt{\frac{r_{j, \varepsilon}}{n}} \mathrm{e}^{-n / r_{\varepsilon}} \sin \frac{n}{r_{0}} \cos \mathbf{K} \cdot \mathbf{n}
\end{aligned}
$$

where the characteristic scales are 


$$
r_{j, \varepsilon}=r_{0}\left(\pi \frac{\varepsilon_{g}-\varepsilon^{(j)}}{\varepsilon-\varepsilon^{(j)}}\right)^{2}, \quad r_{\varepsilon}=r_{0} \frac{\xi_{0}^{2}}{\delta^{2}}, \quad r_{0}=\frac{\hbar v_{\mathrm{F}}}{\xi_{0}} .
$$

A similar behavior with two oscillating factors in effective interimpurity interactions was previously indicated for the impurity states within superconducting gap in iron pnictides [23, 224], where a faster cosine factor had Fermi wavelength. But, the present case is simplified by the $\mathbf{K}$-point specific property that, for all separations $\mathbf{n}$ between lattice sites of the same $j$ th type, $\cos ^{2} \mathbf{K} \cdot \mathbf{n}$ only takes the values $\sigma=1$ and $1 / 4$ (with respective weights $p_{\sigma}=1 / 3$ and $2 / 3$ ), whose contributions can be then simply added up in Eq. (6.14). These partial contributions are obtained by subsequent integrations [23], first over the poles of fast oscillating sine and then over its residues with the slow envelope function $F_{j, n, \sigma}^{2}=\sigma r_{j, \varepsilon} \mathrm{e}^{-2 n / r_{\varepsilon}} / n$ :

$$
\begin{aligned}
B_{j} & =\sum_{\sigma} p_{\sigma} \operatorname{Im} \sum_{n>a} \frac{F_{j, n, \sigma}^{2} \sin ^{2}\left(n / r_{0}\right)}{1-F_{j, n, \sigma}^{2} \sin ^{2}\left(n / r_{0}\right)} \\
& \approx \sum_{\sigma} \frac{4 \pi p_{\sigma}}{\sqrt{3} a^{2}} \int_{a}^{r_{\max }} \frac{r d r}{\sqrt{F_{j, r, \sigma}^{2}-1}}
\end{aligned}
$$

where $r_{\max }$ corresponds to $F_{j, r_{\max }}=1$. The latter integration is simplified within the energy range of

$$
\varepsilon^{(j)}-\varepsilon \gg\left(\varepsilon_{g}-\varepsilon^{(j)}\right)^{5 / 4} / \varepsilon_{g}^{1 / 4},
$$

where $r_{j, \varepsilon} \ll r_{\varepsilon}$ so that the exponential factor in Eq. (6.15) is approximately unity for all distances $r<r_{\max } \approx r_{j, \varepsilon}$. In this approximation, the explicit result for the most dispersive subband reads

$$
B_{2}(\varepsilon)=\frac{7 \pi}{64}\left(\frac{r_{2, \varepsilon}}{a}\right)^{2}
$$

with the prefactor resulting precisely from weighting of $\sigma$ values. Then the above-suggested expansion of $\operatorname{det} \hat{G}_{\mathbf{k}}^{-1}$ for $\varepsilon$ closer to $\varepsilon^{(2)}$ than to other $\varepsilon^{(j)}$ (so that all $\Sigma_{j}$ except $\Sigma_{2}$ can be neglected) provides the linewidth,

$$
\Gamma_{2}(\varepsilon) \approx c^{2}\left(\varepsilon^{(2)}-\varepsilon\right) B_{2}(\varepsilon),
$$

valid until $\varepsilon^{(2)}-\varepsilon \lesssim \varepsilon_{g}-\varepsilon^{(2)}$. Upon going farther from $\varepsilon^{(2)}$, we have $r_{j, \varepsilon}<r_{0}$ so that $B_{2}(\varepsilon)$ vanishes and finite $\Gamma_{2}$ values can only result from the higher-order 
GE terms (if not to include, of course, such relaxation processes as by thermal phonons, electron-electron collisions, etc.). From Eq. (6.19), the IRM criterion is reduced to the inequality

$$
c B_{2}(\varepsilon) \ll 1
$$

(agreeing with the GE convergence), and, supposing Eq. (6.17) to be valid, this criterion permits to estimate the mobility edge separation from the $\varepsilon^{(2)}$ level:

$$
\varepsilon^{(2)}-\varepsilon_{c, 2} \sim c^{1 / 4} \sqrt{\frac{W}{2 \xi_{0}}}\left(\varepsilon_{g}-\varepsilon^{(2)}\right) .
$$

All the states with energies closer to $\varepsilon^{(2)}$ than $\varepsilon_{c, 2}$ are localized on certain clusters of second-type impurity centers. The first conclusion from the estimate, Eq. (6.20), is that existence of the impurity subband itself is only assured if its bandwidth $\approx \varepsilon^{(2)}-\varepsilon_{g, 2}$ surpasses the width of localized range around $\varepsilon^{(2)}$. This is fulfilled when the total impurity concentration exceeds the critical value:

$$
\begin{aligned}
c_{c r} & \sim\left(\frac{t_{z}}{W}\right)^{8 / 3}\left(\frac{|U|}{W}\right)^{4 / 3}\left(\frac{V}{W}\right)^{2 / 3} \\
& \times \frac{\left(t_{z}+\sqrt{t_{z}^{2}+V^{2}}\right)\left(2 t_{z}^{2}+V^{2}\right)}{\left(t_{z}^{2}+V^{2}\right)^{2 / 3} t_{z}^{5 / 3}} .
\end{aligned}
$$

[it is obtained by approximating Eq. (6.6) only to its diverging terms]. Smallness of this expression is mainly due to its first three essential factors of interlayer coupling, impurity perturbation, and applied bias, while the last factor stays almost constant for all realistic (not too high) $V$ values. Thus, for the sample choice of $W=20 t_{z},|U|=10 t_{z}$, and $V=2 t_{z}$, we obtain $c_{c r} \sim 1.8 \cdot 10^{-5}$. Then for the example of $c=0.01$ chosen in Fig. 6.3, the mobility edge $\varepsilon_{c, 2}$ extends from $\varepsilon^{(2)}$ to about the distance $\varepsilon_{g}-\varepsilon^{(2)}$, while the dispersion of $\varepsilon^{(2)}(\xi)$ subband is about an order of magnitude bigger (see Fig. 6.4). Finally, from comparison of ranges by Eqs. refeq20 $\left.{ }_{2} 015\right)$ and (6.17), it followsthatthelatteroneforc $>\mathrm{c}_{c r}$ always occurs within the localized range and so the exponential factor in Eq. (6.15) cannot influence the above-obtained estimates. In summary, only the most dispersive impurity subband by the lowest impurity level can be considered to really emerge beyond its mobility edge. Its main specifics in anomalously strong variation of the lifetimes $\tau(\varepsilon)$ along very narrow energy intervals. 


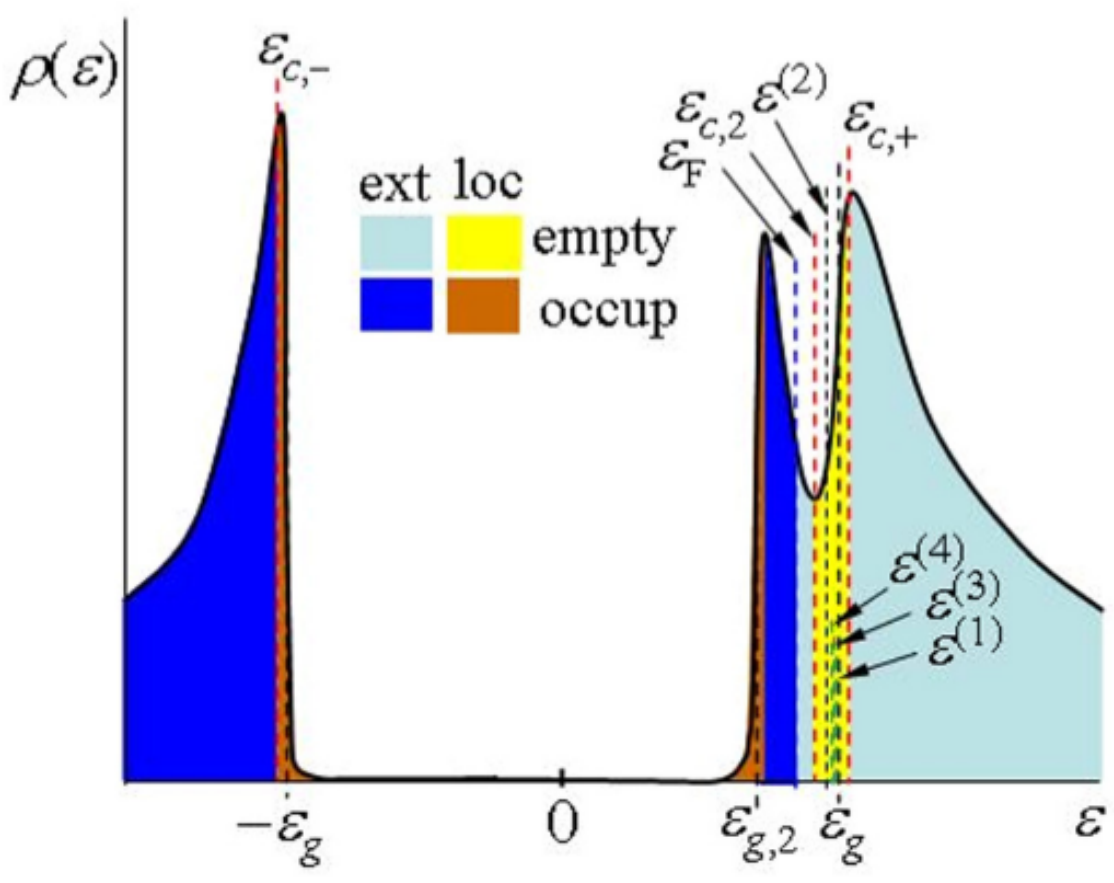

Figure 6.5: Schematics of extended (ext) and localized (loc) ranges in the energy spectrum of bilayer graphene with impurities for the situation like that of Fig. (6.4). Note the position of the Fermi level $\varepsilon_{\mathrm{F}}$ (separating occupied and empty states) with respect to the mobility edges (separating ext and loc states); the narrow impurity band only emerges below the lowest impurity level $\varepsilon^{(2)}$, while the resting $\varepsilon^{j}$ levels get buried within the localized range from $\varepsilon_{c, 2}$ up to $\varepsilon_{c,+}$ (see in text).

As to other formal solutions of Eq. refeq $\left.3_{2} 015\right)$ (analyzedwithinclusionoftheresting $\mathrm{B}_{j}$ ), they are mostly invalidated within the common overlapped range of localized levels that extends up to $\varepsilon_{c,+}$, the mobility edge of the upper main band. The states in this area can be characterized only by their DOS. Though the latter function can not be directly found here from the above defined GEs [Eqs. (6.9) and (??)], it can be plausibly expected to vary smoothly up to the peak near $\varepsilon_{g}$ (Fig. ??) so that the total number of states $\int_{-W}^{W} \rho(\varepsilon) d \varepsilon=4$ is kept.

Similarly, some finer details of the energy spectrum can be found, as, for instance, the rest of mobility edges $\varepsilon_{c, \pm}$ that define the broadened edges of main subbands, and that near the extremum $\varepsilon_{g, 2} \approx \varepsilon^{(2)}\left(\xi_{0}\right)$ of the impurity subband 
(see Fig. ??). Finally, the case of low impurity concentration, $c<c_{c r}$, can be also included when there is no impurity band within the gap, but the localized levels $\varepsilon^{(j)}$ turn to be separately resolved. Since all these data are less relevant for our main practical purpose below, they are left beyond the present scope. Nevertheless, the presented results essentially develop the general picture of quasiparticle spectra in crystals with impurities under external fields [132].

\section{Biased Metal-Insulator Transitions and their Observable Effects}

Now we can pass to the important processes of electric transport in the system with the above-described band spectrum. For simplicity, this consideration is restricted to the case of zero temperature, and the main attention is paid to the position of Fermi level $\varepsilon_{\mathrm{F}}$ and to the lifetime $\tau_{\mathrm{F}}$ of Fermi states under the applied bias control $V$ at given parameters of impurity perturbations $c$ and $U$. The basic condition for the Fermi level,

$$
2 \int_{-\infty}^{\varepsilon_{\mathrm{F}}} \rho(\varepsilon) d \varepsilon=1+c^{\prime}
$$

defines its shift from the zero energy position in the unperturbed system, in order to accommodate the total of $c^{\prime}$ extra carriers per unit cell (brought by impurities themselves and possibly by some external sources). This generally requires knowledge of DOS functions for all the impurity subbands (besides weakly perturbed main subbands). But our main interest here is in finding a possibility for $\varepsilon_{\mathrm{F}}$ to be located within the most dispersive impurity band $\varepsilon^{(2)}(\xi)$, so we focus on the related DOS, especially in proximity to this band termination $\varepsilon_{g, 2}$ (Fig. ??). An important simplification of this task is obtained by noting that for this energy range all the self-energies $\Sigma_{j}$ in Eq. (6.10) can be taken as constants, small enough compared to the gap width; thus the solutions of the dispersion equation [Eq. (6.3)] almost reproduce here the non-perturbed $\varepsilon_{2}(\xi)$ band within accuracy to a constant shift of its edge from $\varepsilon_{g}$ to $\varepsilon_{g, 2}$ (see also Fig. 6.4), just due to the common effect of all $\Sigma_{j}$. The resulting DOS function,

$$
\rho_{2}(\varepsilon) \approx \frac{2 \varepsilon}{W^{2}} \frac{t_{z}^{2}+V^{2}}{\delta^{2}}
$$

at $0<\varepsilon-\varepsilon_{g, 2} \lesssim \varepsilon^{(2)}-\varepsilon_{g, 2}$ defines from Eq. (6.21) the Fermi level $\varepsilon_{\mathrm{F}}$ position by the equation 


$$
c^{\prime} \approx\left(\frac{2}{W}\right)^{2} \sqrt{\left(t_{z}^{2}+V^{2}\right)\left(\varepsilon_{g}^{2}-\varepsilon_{\mathrm{F}}^{2}\right)}
$$

Let $c_{\max }^{\prime}$ be the maximum permitted amount of carriers such that $\varepsilon_{\mathrm{F}}$ stays within the conducting range. Then, for the case of Fig. 6.4, this value results $c_{\text {max }}^{\prime} \approx 4 \cdot 10^{-3}$, that is, somewhat lower than the proper impurity concentration $c=10^{-2}$ in this case. Nevertheless, conduction through the impurity band can be realized if $c^{\prime}$ is brought below the indicated limit of $c_{\text {max }}^{\prime}$, e.g., by external compensation of a part of charge carriers [34]. Once this is assured, one can then strongly change the conductivity by raising the applied $V$, since the localized range width by Eq. (6.19) grows with bias faster than $\propto V^{2 / 3}$ against the almost bias insensitive (at $V \lesssim V_{c r}$ ) width of the impurity band, while the Fermi level $\varepsilon_{\mathrm{F}}$ goes to the band edge $\varepsilon_{g, 2}$ slower than $\propto V^{-2}$. Then the faster advancing mobility edge $\varepsilon_{c, 2}$ will finally cross $\varepsilon_{\mathrm{F}}$ at some bias $V_{M-I}$, giving rise to a Mott metal-insulator transition and vanishing conductivity. Thus, for the proposed choice of $U=-W / 2=-10 t_{z}$ and $c^{\prime}=3 \cdot 10^{-3}$, we obtain $V_{M-I} \approx 0.87 \mathrm{eV}$. In this course, at $V \rightarrow V_{M-I}$, conductivity can vary by orders of magnitude, when we drive the Fermi inverse lifetime $\tau_{\mathrm{F}}^{-1} \sim \Gamma_{2}\left(\varepsilon_{\mathrm{F}}\right) / \hbar$ close to divergence, under very tiny variations (say, some meV) of bias. This indicates a tremendous potentiality of such type of doped semiconducting systems in comparison with traditional materials.

Besides their evident field transistor applications, critical effects by the biased Mott transition can be also expected in other observable properties of this doped system, for instance, in its optical response at the frequency $\omega_{i, b} \approx$ $\left(\varepsilon^{(2)}+\varepsilon_{g}\right) / \hbar$ of transition from the top of occupied $-\varepsilon_{2}(\xi)$ band and the Fermi states of impurity $\varepsilon^{(2)}(\xi)$ band (like the case formerly considered by the authors for doped superconducting iron pnictides [224]), which can be switched on and off by tiny variation of the bias.

At last, with further growing bias, the collapse of upper and lower mobility edges within the impurity band and the aforementioned Anderson transition to fully localized in-gap spectrum can be realized. From Eq. (6.20) at $V \lesssim t_{z}$, this bias value estimates as $V_{\mathrm{A}} \sim c^{3 / 2} W^{7}|U|^{-2} t_{z}^{-4}$, though this analytic expression applies only (at moderate $|U|$ ) for as low impurity concentrations as $c \lesssim 10^{-5}$ - However, a numerical analysis with use of full Eq. (6.6) shows that $V_{\mathrm{A}}$ remains attainable up to $c \sim 10^{-2}$ as well. This transition can also produce observable effects; in this case the collapse of narrow impurity band would lead to a dramatic drop of the plasmonic resonance frequency [227]. 


\section{Discussion and Conclusions}

The above main conclusion regarding the possibility of attaining extensive control of electrical conduction through very slight variations of applied potential implies, of course,many additional factors to be taken into account. They can be indicated both from the fundamental and practical sides. Thus, the theoretical approach used is restricted to a simple model of impurity perturbation by a single on-site parameter,and some elaboration of it could be done involving, for instance, perturbations of hopping parameters. These kinds of analyses are known for traditional doped semiconductors and also have demonstrated possibilities for similar impurity bands near localized impurity levels at high enough impurity concentrations. Notably, for those materials, the Lifshitz perturbation model was found to be the most restrictive for such effects, for instance, due to unrealistically high critical concentrations, of the order of unity or even more [unlike that in Eq. (6.19)]. This permits the expectations that modifications of the present Lifshitz model, as in Ref. [212] for single impurity at a gapless spectrum, or using the Anderson hybrid model [221] as in Ref. [228] (provided all the technical aspects be assured), will not essentially change the physical behavior of the system. On the other hand, there are yet many properties of this simple model that can be further studied, for instance, the possibilities to realize multiple conducting impurity subbands and subsequent processes of multiple switching between them, including, e.g., optical transitions under electrical biasing. Of course, a more realistic approach should also take account of topological defects (see beginning of Sec. III) as well as the above-mentioned Coulomb interactions, thermal effects, etc. Generally, this would require the impurity band structure to exceed a certain "background" relaxation level that could be achieved by varying either the impurity sort (that is, $U$ parameter) and concentration or/and the applied bias $V$. Finally, similar impurity multiband effects can be also sought in other atomically multilayered systems, such as those mentioned in the Introduction, where a special focus might be put on the tuned band gap in silicene bilayers (yet wider than in bilayer graphene [206]) or even on single layers of buckled silicene or germanene [229].

As to the practical issues, first of all, rather strict conditions on fabrication of the basic doped bilayered system are in order, perhaps mainly aimed to minimize all the "foreign" defects vs the chosen dopants, but the next requirement to control the levels of dopants (and possibly their compensating species) within fractions of percent should not be a real problem for modern nanoelectronics. Special attention is also required for precise control and manipulation of the applied bias $V$, particularly in exploring possibilities to realize its near- 
critical and super-critical regimes, like those indicated in the above analysis. Finally, the practical arrangement of an experimental transistor-type setup based on the suggested conductivity control by tiny impurity subbands would perhaps require some specific technical solutions. However, they do not look too difficult to be found in the available engineering depository. Thus a fair hope exists for this theoretical proposal to be realized in a practical device.

In conclusion, the effects of localized on-site perturbations by rather disperse impurities on a bilayered graphene system under the applied electrical bias between the layers are analytically considered using the Green's function techniques adapted for a multiband electronic system. Thus the conditions for different types of localized impurity levels to appear within the bias-induced band gap in the electronic spectrum of this system and then extension of these levels into specific narrow energy bands at impurity concentrations above certain characteristic values are demonstrated. The analysis of these processes demonstrated their similarities to those known from literature on various crystalline materials with impurities. Also, some specifics of the present system were shown in considerable bias dependencies of impurity bands and of critical concentrations for their formation. These dependencies can be further treated to provide some specific phase diagrams in variable "bias concentrations", as it took place in antiferromagnetic insulators where such diagrams in variable "magnetic field concentration" were quite informative [132]. A practical application of the described electronic band structure is suggested in a form of highly sensitive bias control on the system's conductivity through the impurity subband when brought close to a regime of bias-controlled Mott metal-insulator transition.

\section{Appendix}

In calculation of the interaction function, Eq. (6.15), the essential task consists in the integration as follows:

$$
\begin{aligned}
& \frac{1}{2 N} \sum_{\mathbf{k}} \mathrm{e}^{i \mathbf{k} \cdot \mathbf{n}}\left(G_{\mathbf{k}}^{(0)}\right)_{j j}=\frac{2 \cos \mathbf{K} \cdot \mathbf{n}}{W^{2}} \int_{0}^{W} J_{0}\left(\frac{\xi n}{\hbar v_{\mathrm{F}}}\right) \\
& \times \quad \frac{\left(N_{j}(\varepsilon)-\xi^{2}\right) \xi d \xi}{\left(\xi^{2}-\xi_{1}^{2}\right)\left(\xi^{2}-\xi_{2}^{2}\right)}
\end{aligned}
$$

where $J_{0}$ is the zeroth order Bessel function, $\xi_{1,2}^{2}=\varepsilon^{2}+\varepsilon_{2}^{2} \pm \delta^{2}(\varepsilon)$ are the complex poles of $\operatorname{det} \hat{G}_{\mathbf{k}}^{(0)}$ in $\xi$ variable, and all $\left|N_{j}(\varepsilon)\right| \sim \varepsilon_{g}^{2}$ [as seen from Eq. (6.6]). Since this integral is fast converging after $\xi \gtrsim \varepsilon_{g}$, its upper limit can be 
safely extended to infinity. Then, after expanding the factor besides the Bessel function in simple fractions,

$$
\frac{N_{j}(\varepsilon, \xi)}{\left(\xi^{2}-\xi_{1}^{2}\right)\left(\xi^{2}-\xi_{2}^{2}\right)}=\frac{N_{j}(\varepsilon)-\xi_{1}^{2}}{\xi^{2}-\xi_{1}^{2}}-\frac{N_{j}(\varepsilon)-\xi_{2}^{2}}{\xi^{2}-\xi_{2}^{2}},
$$

the Hankel-Nicholson integration formula can be applied to each of them:

$$
\int_{0}^{\infty} \frac{J_{0}(x) x d x}{x^{2}+z^{2}}=K_{0}(z)
$$

with the zeroth order MacDonald function $K_{0}$, valid for complex $z$ if $\operatorname{Re} z>0$ (Ref. [197]). The $z$-arguments related to the terms in Eq. (6.26), can be defined as $z_{1,2}^{2}=-\xi_{1,2}^{2}\left(n / \hbar v_{\mathrm{F}}\right)^{2}$ and the above requirement will read $\operatorname{Re} \sqrt{-\xi_{1,2}^{2}}>0$. For the relevant energy range $0<\varepsilon_{g}-\varepsilon \ll \varepsilon_{g}$, we can use the choices $\sqrt{-\xi_{1,2}^{2}}=$ $\sqrt{\sqrt{\gamma^{2}(\varepsilon)-\varepsilon^{2}-\varepsilon_{2}^{2}}} \mp i \sqrt{\sqrt{\gamma^{2}(\varepsilon)+\varepsilon^{2}+\varepsilon_{2}^{2}}}$. At last, for relevant distances $n \gtrsim$ $r_{0}$, the resulting $K_{0}\left(z_{1,2}\right)$ have big enough arguments, $\left|z_{1,2}\right|=\left|n \xi_{1,2} / \hbar v_{\mathrm{F}}\right| \gtrsim 1$, to use their asymptotics: $K_{0}\left(z_{1}\right) \approx-\sqrt{2 / \pi z_{1}} \mathrm{e}^{-z_{1}}$ and $K_{0}\left(z_{2}\right) \approx \sqrt{2 / \pi z_{2}} \mathrm{e}^{-z_{2}}$. Then, taking account of all prefactors besides these expressions present in Eqs. (6.15) and (6.26), we arrive at the final result of Eq. (6.15). 

Chapter 7

\section{Electronic and Phonon \\ Instabilities in Bilayer Graphene under Applied External Bias}




\title{
ScienceDirect
}

\section{Electronic and Phonon Instabilities in Bilayer Graphene under Applied External Bias}

\author{
E.L. Silva ${ }^{\mathrm{a}, \mathrm{b}^{*}}$, M.C. Santos ${ }^{\mathrm{c}}$, J.M. Skelton ${ }^{\mathrm{d}}$, Tao Yang ${ }^{\mathrm{e}}$, T. Santos ${ }^{\mathrm{f}}$, S.C. Parker ${ }^{\mathrm{b}}$, A.Walsh ${ }^{\mathrm{g}}$ \\ ${ }^{a} I D F$, and MALTA Consolider Team, Universitat Politècnica de València, 46022 València, Spain \\ ${ }^{b}$ Department of Chemistry, University of Bath, Bath BA2 7AY, United Kingdom \\ ${ }^{c}$ Department of Physics, University of Coimbra, 3004-516 Coimbra, Portugal \\ ${ }^{d}$ School of Chemistry, University of Manchester, Oxford Road, M13 9PL, United Kingdom \\ ${ }^{e}$ Key Laboratory of Biofuels, Qingdao Institute of Bioenergy and Bioprocess Technology, Chinese Academy of Sciences, Qingdao, 26610, China \\ ${ }^{f}$ CICECO, Department of Physics, University of Aveiro, 3810-193 Aveiro, Portugal \\ ${ }^{g}$ Department of Materials, Imperial College London, London SW7 2AZ, United Kingdom
}

\begin{abstract}
We have performed electronic-structure and lattice-dynamics calculations on the AB and AA structures of bilayer graphene. We study the effect of external electric fields and compare results obtained with different levels of theory to existing theoretical and experimental results. Application of an external field to the $\mathrm{AB}$ bilayer alters the electronic spectrum, with the bands changing under bias from a parabolic to a "Mexican hat" structure. This results in a semi-metal-to-semiconductor phase transition, with the size of the induced electronic band-gap being tuneable through the field strength. A reduction of continuous symmetry from a hexagonal to a triangular lattice is also evidenced through in-plane electronic charge inhomogeneities between the sublattices. When spin-orbit coupling is turned on for the $\mathrm{AB}$ system, we find that the bulk gap decreases, gradually increasing for larger intensities of the bias. Under large bias the energy dispersion recovers the Mexican hat structure, since the energy interaction between the layers balances the coupling interaction. We find that external bias perturbs the harmonic phonon spectra and leads to anomalous behaviour of the out-of-plane flexural ZA and layer-breathing ZO' modes. For the AA system, the electronic and phonon dispersions both remain stable under bias, but the phonon spectrum exhibits zone-center imaginary modes due to layersliding dynamical instabilities.

(C) 2018 Elsevier Ltd. All rights reserved.

Selection and peer-review under responsibility of the scientific committee of 11th International Conference on Advanced Nano Materials.
\end{abstract}

Keywords: Bilayer graphene; electric field; electronic properties; lattice dynamics

* Corresponding author. Tel.: +34-96-387-70-00; fax: +34- 96-387-90-09.

E-mail address: esdasil@idf.upv.es

2214-7853 (C) 2018 Elsevier Ltd. All rights reserved.

Selection and peer-review under responsibility of the scientific committee of 11th International Conference on Advanced Nano Materials. 


\title{
Electronic and Phonon Instabilities in Bilayer Graphene under Applied External Bias
}

\author{
E.L. Silva ${ }^{a, b}$, M.C. Santos ${ }^{c}$, J.M. Skelton ${ }^{d}$, Tao Yang ${ }^{e}$, T. Santos ${ }^{f}$, S.C. \\ Parker $^{b}$, A.Walsh ${ }^{g}$ \\ ${ }^{a}$ IDF, and MALTA Consolider Team, Universitat Politècnica de València, \\ 46022 València, Spain \\ ${ }^{b}$ Department of Chemistry, University of Bath, Bath BA2 7AY, United \\ Kingdom \\ ${ }^{c}$ Department of Physics, University of Coimbra, 3004-516 Coimbra, Portugal \\ ${ }^{d}$ School of Chemistry, University of Manchester, Oxford Road, M13 9PL, \\ United Kingdom \\ ${ }^{e}$ Key Laboratory of Biofuels, Qingdao Institute of Bioenergy and Bioprocess \\ Technology, Chinese Academy of Sciences, Qingdao, 26610, China \\ ${ }^{f}$ CICECO, Department of Physics, University of Aveiro, 3810-193 Aveiro, \\ Portugal \\ ${ }^{g}$ Department of Materials, Imperial College London, London SW7 2AZ, \\ United Kingdom
}

We have performed electronic-structure and lattice-dynamics calculations on the $\mathrm{AB}$ and $\mathrm{AA}$ structures of bilayer graphene. We study the effect of external electric fields and compare results obtained with different levels of theory to existing theoretical and experimental results. Application of an external field to the $\mathrm{AB}$ bilayer alters the electronic spectrum, with the bands changing under bias from a parabolic to a "Mexican hat" structure. This results in a semimetal-to-semiconductor phase transition, with the size of the induced electronic band-gap being tuneable through the field strength. A reduction of continuous symmetry from a hexagonal to a triangular lattice is also evidenced through inplane electronic charge inhomogeneities between the sublattices. When spinorbit coupling is turned on for the $\mathrm{AB}$ system, we find that the bulk gap decreases, gradually increasing for larger intensities of the bias. Under large bias the energy dispersion recovers the Mexican hat structure, since the energy interaction between the layers balances the coupling interaction. We find that external bias perturbs the harmonic phonon spectra and leads to anomalous behaviour of the out-of-plane flexural ZA and layer-breathing ZO' modes. For the AA system, the electronic and phonon dispersions both remain stable under bias, but the phonon spectrum exhibits zone-center imaginary modes due to layer-sliding dynamical instabilities. 


\section{Introduction}

Among the numerous derivatives of the monolayer graphene (MLG) system, special interest has been given to the multi-layer allotropes [21] in particular Bernal bilayer graphene with AB stacking (AB-BLG) [230]. Like ML graphene, BL graphene also displays unconventional properties [10] that are relevant to technological developments including tunnelling field-effect transistors [231] and high-rate lithium-sulphur batteries [232, 233], nanophotonics [234], sensor modelling [235], among others. These properties originate from the weak coupling between layers, which allows for the properties of the base ML graphene material to be retained. Despite the similarities between ML and BL graphene, there are also significant differences between the two allotropes. ML graphene shows a linear band dispersion near the Fermi energy, and the valence and conduction bands touch at the K-point (the Dirac point), yielding the characteristic dispersion of relativistic massless Dirac electrons [236, 237]. For unbiased AB-BLG, on the other hand, the interlayer coupling produces a parabolic-like band structure around the K-point. These different features result in a vanishing of the density-of-states (DOS) at the Fermi energy for the MLG [237], in contrast to a finite DOS evidenced in the AB-BLG. Another characteristic feature of AB-BLG is the behaviour of the system when an electric field is applied normal to the layers. It has recently been shown that biased AB-BLG can form a Wigner crystal, due to the existence of different kinetic-energy dispersions at different electron densities [238]. The energy band gap can be tuned in proportion to the intensity of the applied bias [230], and two distinct zero-temperature quantum phases at different electron densities can be formed $[238,239]$. For the AB-BLG system, the presence of significant spin-orbit coupling (SOC) effects has been evidenced by topological-insulator behaviour with a finite spin Hall conductivity [240]. Moreover, it has also been shown that biased BLG may exhibit two topologically-distinct phases depending on the intensity of the Rashba spin-orbit coupling (RSOC) [241]. For weak coupling, the system exhibits a quantum-valley Hall state, which can then transition to a topological insulator in the presence of strong coupling effects. It is possible to transition between these two phases by tuning the applied electric field [241]. In the presence of strong RSOC, and for sufficiently short-range electron-electron interactions, the system minimises its energy by adopting broken-symmetry states (mostly those which break rotational symmetry) in the limit of low densities [242]. These instabilities occur due to the energy dispersion having a minimum in a region of momentum-space which is bounded by two concentric circles with finite radius (annuli) [243]. Moreover, distortions to the Fermi surface, resulting from a momentum-space change in the Fermi radius (a Pomeranchuk instability) can reduce the lattice symmetry 
and lead to spontaneous longitudinal currents [243]. Another stacking arrangement of BL graphene, which coexists with the AB stacking, is the AA structure where the carbon atoms are positioned directly above each other in consecutive layers. The electronic properties differ from those of AB-BLG due to the stacking arrangement. The AA stacking has been experimentally observed in disordered or pregraphitic carbon, also known as turbostratic graphite, and can be distinguished from ML graphene by so-called tilting experiments [244, 245]. However, as the space groups of AA-BLG and MLG are the same (P6/mmm), similarities between the two are difficult to predict. Between the two stacking environments, the AB stacking is the most energetically favourable form, and is separated from the AA stacking by a small energy barrier. Despite its instability, AA-BLG has started to receive significant attention. The AA configuration shows unusual electronic properties, with two degenerate electronic and hole bands crossing at the Fermi energy [246]. This electronic structure supports several electron and electron-phonon instabilities, which include, among others, a shear-shift instability [246]. It has further been observed that small perturbations can destabilize the degenerate spectrum and generate an excitonic gap $[246,247]$. While the AB-BLG system is well studied both experimentally and theoretically, comparatively less attention has been given to the AA stacking. In the present work, we aim to provide more insight into the electronic and vibrational properties of biased AA-BLG, and to make a comparison to the AB-system, by employing first-principles simulation techniques. We find that while the $\mathrm{AB}$ system presents variations on the electronic densities as a function of the applied bias, we observe that the AA system remains unaltered when an electric field is applied. SOC effects are also considerable for the biased AB-system, with the band-gap presenting different scaling behaviours according to the field intensities. The phonon dispersions of the biased $\mathrm{AB}$ system shows instabilities of the out-of-plane acoustic and optic modes, when compared to the stability of these modes for the unbiased system. On the other hand, phonon dispersions of the AA system remain stable under bias, but the phonon spectrum exhibits a zone-centre imaginary mode resulting form the shear-mode instability.

\section{Theoretical Framework}

We study the electronic structure of the two different stacking environments of the BLG system (crystal structure of AB- and AA-BLG presented in Fig. 7.1) using density-functional theory (DFT) with the Local-Density Approximation (LDA) functional. An external electric field is applied in the direction of the interlayer plane with variable magnitude. Lattice dynamics are performed 
a)

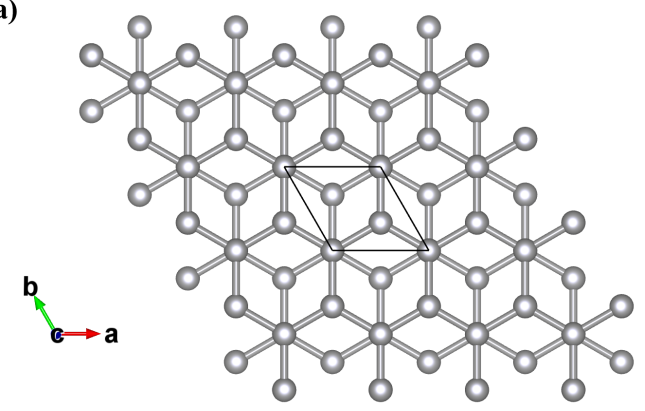

b)

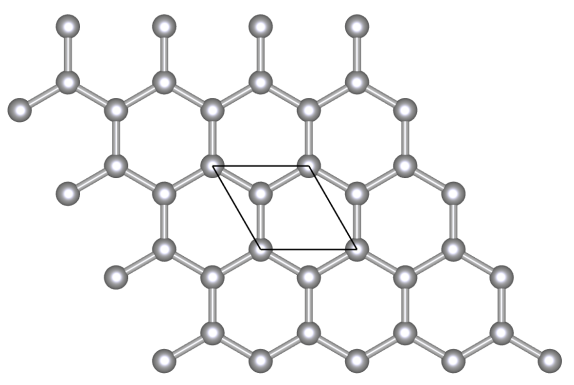

Figure 7.1: Supercell of the AB- (space group , n. 164; a) and AA-BLG (space group $\mathrm{P} 6 / \mathrm{mmm}$, n. 191; b) systems, where the black line shows the unit-cell. BLG consists of two coupled monolayers of carbon atoms, each with a honeycomb crystal structure. In order to satisfy the translational and symmetry properties of the Bravais lattice, the honeycomb lattice can be seen as two triangular sublattices, mathematically labelled as inequivalent A and $\mathrm{B}$ lattices, each of which contains two atoms in the unit cell within each $\mathrm{C}$ sheet, with atom $\left[\mathrm{a}_{1}, \mathrm{a}_{2}\right] \in \mathrm{A}$ and $\left[\mathrm{b}_{1}, \mathrm{~b}_{2}\right] \in \mathrm{B}$ for layer 1 and 2 . The layers of the AB-BLG are arranged in such a way that one of the atoms from the lower-layer b1 is directly below atom $\mathrm{a}_{2}$ from the upper layer, and the remaining two atoms, $a_{1}$ and $b_{2}$, are shifted from each other by a vector displacement [237]. For the AA-BLG, the carbon atoms are aligned in the consecutive layers, directly above/below each other ( $a_{1}$ with $a_{2}$ and $b_{1}$ with $b_{2}$ ).

within the harmonic approximation, which yields phonon frequencies and the constant-volume terms in the free energy from lattice vibrations.

\section{II.1 Density Functional Theory}

Electronic-structure calculations were performed within the pseudopotential plane-wave density-functional theory (DFT) framework, as implemented in the Vienna Ab-initio Simulation Package [248, 249, 250] code. The Ceperley and Alder form of the Local-Density Approximation (LDA) functional, parameterized by Perdew and Zunger [72] was used in conjunction with projector augmented-wave (PAW) pseudopotentials [69, 251]. We selected the LDA functional because it is known to perform well at capturing the interlayer distance in graphite and multi-layer graphene allotropes, as well as the essential physics of the electronic structure, and also performs well for calculating interatomic force constants and phonon frequencies [252, 253]. A plane-wave cut-off of $800 \mathrm{eV}$ was applied in all calculations; although convergence of the electronic structure was attained at a lower cut-off of $600 \mathrm{eV}$, a higher value 
was chosen to improve the description of the structural parameters and forces, which is important for accurate lattice-dynamics calculations [254]. The Brillouin zone (BZ) was sampled with $\Gamma$-centred Monkhorst-Pack meshes [255] with $44 \times 44 \times 1$ and $90 \times 90 \times 1$ subdivisions for AA- and AB-BLG respectively. It was found necessary to employ the denser k-point mesh for the AA-BLG model due to differences in the DFT electronic band structure relative to the spectra expected from tight-binding theory $[230,256]$. The vacuum spacing between periodic images along the $\mathrm{Z}$ direction was set to $15 \AA$ for both configurations, and dipole corrections to the potential were applied to avoid interactions between periodic images. Lattice-dynamics calculations were carried out using the Parlinski-Li-Kawazoe supercell finite-displacement method [81, 82], which is implemented in the Phonopy $[257,258]$ package; a detailed description of the theoretical implementation can be found in Refs. [254] and [83]. The interatomic force constants were obtained by performing single-point force calculations on a series of symmetry-inequivalent displaced structures and fitting the resulting force/displacement curves to a harmonic function. VASP was used as the force calculator [82] and the calculations were performed on $4 \times 4 \times 1$ supercells using a reduced k-point sampling mesh of $12 \times 12 \times 1$ for both phases. For the calculations under bias, the electric field was applied during the forceconstant calculations. To construct the phonon density of states, the phonon frequencies were sampled on an interpolated $48 \times 48 \times 1$ q-point mesh. A nonanalytical correction (NAC) was applied when computing the phonon-band dispersion [259] to correct for long-range Coulomb interactions. The requisite Born effective-charges and static dielectric constant were computed using the density-functional perturbation theory (DFPT) routines implemented in VASP [260]. Convergence of these quantities required increasing the k-point mesh for the AB system up to $80 \times 80 \times 1$, whereas for the AA system the $90 \times 90 \times 1$ mesh was found to be sufficient. A bias was applied in the calculations as an external electrostatic field in the $\mathrm{Z}$ direction and geometries were re-optimised with different intensities of the field. Born effective-charges and dielectric tensors were calculated by considering the field perturbations. For the lattice-dynamics calculations, the bias was also applied during the calculations of the force constants. 


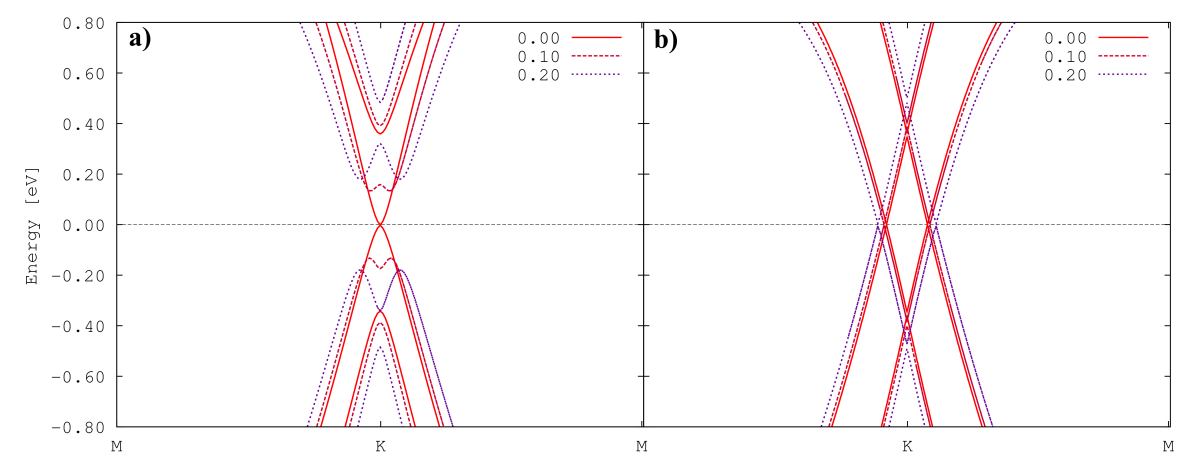

Figure 7.2: Low-energy DFT-LDA electronic band-structure of bilayer graphene with AB (a) and AA (b) stacking arrangements. Each dispersion is shown at different applied field intensities (label units given in $\mathrm{eV} / \AA$ ).

\section{Results and Discussion}

The lattice parameters obtained within the LDA are $\mathrm{a}_{0}=2.42 \AA$ and $\mathrm{c}_{0}=6.69 \AA$ for the $\mathrm{AB}$ system, and $\mathrm{a}_{0}=2.45 \AA$ and $\mathrm{c}_{0}=6.67 \AA$ for the AA system. The intralayer distance ( $\mathrm{C}-\mathrm{C}$ bond lengths) are on the order of $1.41 \AA$ in both stacking environments, and the interlayer distance was calculated to be $3.35 \AA$ and $3.34 \AA$ for the $\mathrm{AB}$ and $\mathrm{AA}$ configurations, respectively. The parameters for AB-BLG are in agreement with those discussed in Ref. [261], where the calculations were also performed with DFT-LDA (intra-layer distance of $1.41 \AA$ and interlayer distance of $3.31 \AA$ ). The present interlayer parameters also compare well to experimental results, where for the Bernal graphite the value of $3.35 \AA$ [262] was observed. However, for the AA-BLG the present interlayer distance is found to be slightly lower than results found in literature: $3.59 \AA$ from DFTLDA calculations [261], and $3.55 \AA$ from experimental observations on the AA graphite structure [263].

\section{III.1 Electronic Spectrum from a Density-Functional Perspective}

To study the electronic structure, we calculated the low-energy band dispersions using LDA-DFT with three intensities of applied electric field. The results are presented in Fig. 7.2. For the AB-BLG configuration (Fig. 7.2.a), 
(a)

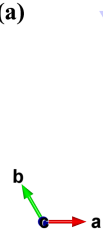

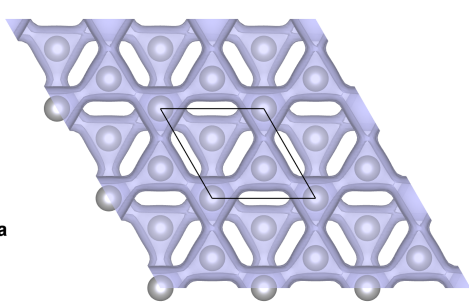

(b)

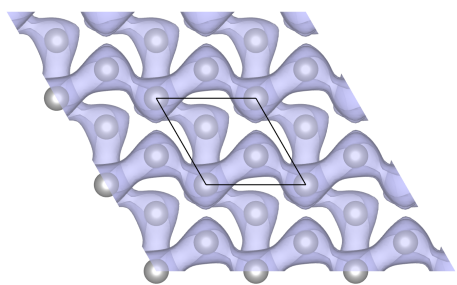

Figure 7.3: Isosurfaces (value defined at 0.016) of the electron charge densities around the Fermi energy for the AB-BLG system without (a) and with (b) an applied bias (electric field intensity of $0.05 \mathrm{eV} / \AA)$.

when $\mathrm{E}=0 \mathrm{eV} / \AA$, a zero-gap parabolic dispersion around the $\mathrm{K}$-point is observed. The LDA-DFT electronic dispersion for the AB system shows similar features to the band-structure obtained from the tight-binding Hamiltonian [256]. When a finite electric-field is applied perpendicular to the graphene layers in AB-BLG, the two layers are subject to inequivalent potentials. This effect breaks the inversion symmetry, resulting in the opening of a single-electron gap [230] at the K-point, which can be tuned up to mid-infrared energies ( 300 $\mathrm{meV}$ ) [202]. A spontaneous translation symmetry breaking also occurs, resulting in a charge separation between the inequivalent sublattices with spatial in-plane charge inhomogeneities [238, 9]. Fig. 7.3 plots the electron charge densities of AB-BLG in the vicinity of the Fermi energy, inspection of which reveals differences between the isosurfaces without (a) and with (b) a bias applied. In the unbiased system (Fig. 7.3.a), the charge densities show hexagonal symmetry, indicating homogeneous electron delocalisation between the sublattices. On the other hand, when an interlayer electric field is applied (Fig. 7.3.b), redistribution of electron densities leads to charge separation between the A and B sublattices, leading to in-plane charge inhomogeneities [238].

The AA-stacking environment differs from the Bernal system by having a linear dispersion with two bands crossing each other at the Fermi energy [237]. Application of an external field does not alter the width of the band gap, and electronic structure remains qualitatively the same. This single-electron property seems to be quite stable to external bias both in the LDA calculations and also with a tight-binding Hamiltonian [237]. These results are consistent with the electronic dispersion calculated with the tight-binding method [256], although, as noted above, obtaining a fully-converged dispersion from the DFT calculations required very dense k-point sampling. This is because the band crossing does not occur at a high-symmetry k-point, and thus a dense mesh 


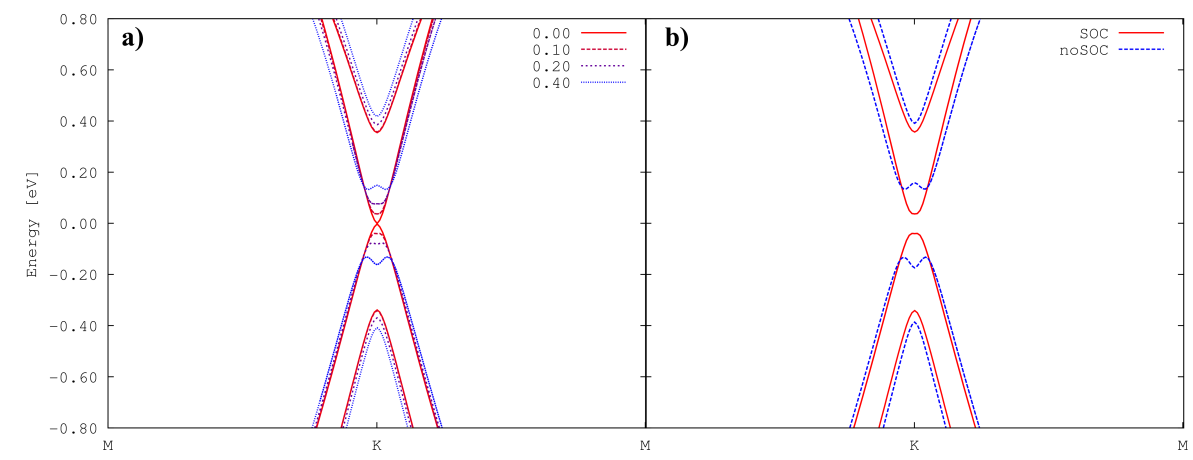

Figure 7.4: Low-energy DFT-LDA electronic band-structure of AB-stacked bilayer graphene. (a) Dispersions with different intensities of an applied external interlayer electric field, calculated with spin-orbit coupling. (b) Dispersions of a biased system $(\mathrm{E}=0.20 \mathrm{eV} / \AA)$ with and without spin-orbit coupling included. Field strengths are given in $\mathrm{eV} / \AA$.

is required in order to include sufficient sampling around the feature to accurately represent the bands in the vicinity of the Fermi energy. Under bias, the dispersion relations of the $\mathrm{AB}$ system show a "Mexican hat" structure $[230,264]$. With increasing field intensity, the width of the gap increases and the radius of the hat feature widens, with the two minima getting progressively further apart from the K-point [230]. This behaviour is consistent with the results from Ref. [238], which suggest that regions of the dispersion should exhibit different scaling behaviour as a function of momentum [238]. Moreover, controlling the magnitude of the gap through additional screening with a transverse electric field will afford control over the density of electrons [214]. Fig. 7.4.a shows the electronic band-structure when spin-orbit coupling (SOC) is included in the calculations. In the present study, we find that the bulk gap decreases when SOC is turned on, and then increases gradually for increasing field intensities (Fig. 7.4.a) under large filed intensities $(\sim 0.4 \mathrm{eV} / \AA)$, the energy dispersion recovers the Mexican hat structure, since the instability occurring at the Fermi-surface competes with the SOC interaction; the energy interaction between the layers balances the coupling interaction. Moreover, Ref. [231] reported that the gap vanished as the SOC parameter increases, and that on further increasing the coupling parameter it then reopens with a behaviour characteristic of a band inversion, thus suggesting a topological phase transition [241]. However, since the model employed in [241] is different from the computations carried out for the presented work, a direct comparison between the two sets of results is not straightforward. 


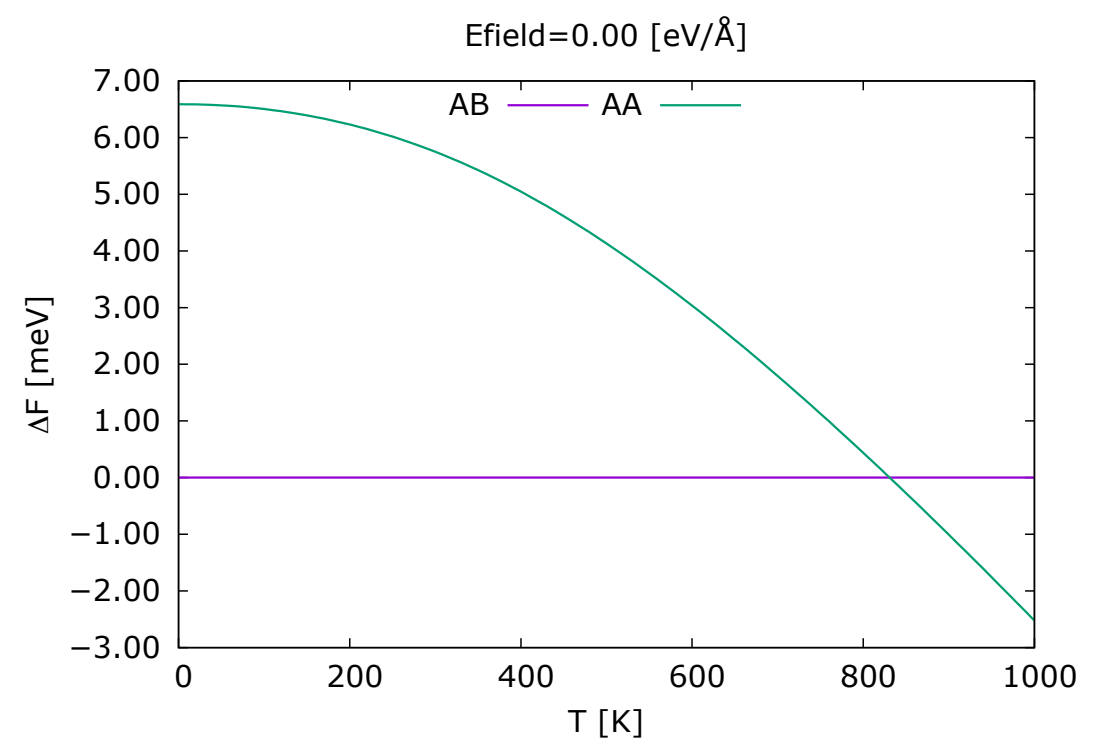

Figure 7.5: Relative free energy (Helmoltz) of the two graphene stacking environments, $\mathrm{AB}$ and $\mathrm{AA}$ (no external electric-bias is applied). The $\mathrm{AB}$ arrangement is calculated to be the most energetically-stable structure up to $800 \mathrm{~K}$.

\section{III.2 Structural Instabilities of Bilayer Graphene - Lattice-Dynamics}

Fig. 7.5 shows the constant-volume (Helmoltz) free energy of the AB and AA bilayer systems calculated without an applied bias. The energies are referenced to the lowest energy structure, which in the present calculations is the $\mathrm{AB}$ system. Our calculations indicate that the AA system is energetically unstable with respect to the $\mathrm{AB}$ phase up to approx. $800 \mathrm{~K}$, above which the AA stacking becomes lower in energy. The calculated in-plane phonon dispersion agrees well with the experimental measurements on graphite presented in [265], apart from a small shift of the higher-frequency TO and LO modes. LDA calculations frequently overestimate the energies of higher-frequency phonons, but despite this difference the characteristic features of the phonon dispersion are well reproduced. Fig. 7.6 compares the phonon dispersions of the two stacking modes. Both stacking configurations have similar mode characters, 
although differences emerge at the zone-centre. For both systems and at low q-vectors, the in-plane transverse acoustic (TA) and longitudinal acoustic (LA) modes show linear dispersions (Fig. 7.6) [256, 266]. Moreover, the AB-system presents a doubly-degenerate LA mode has zero frequency at the $\Gamma$-point, the TA mode (also known as the shear-mode) has a non-zero frequency at the zonecenter [266] $(\nu=0.82 \mathrm{THz})$ (Fig. 7.6). The ZA mode is the flexural acoustic mode, which corresponds to out-of-plane, in-phase atomic displacements. In contrast to the TA and LA modes, the ZA branch shows a parabolic dispersion, i.e. $\sim \mathbf{q}^{2}$ (Fig. 7.6 ) $[256,266,267,268]$, close to the $\Gamma$-point, indicating a low group velocity [267] and being a characteristic feature of layered materials $[266,267]$. The existence of a flexural mode is also a signature of $2 \mathrm{D}$ systems, and in particular is a mode which is typically found in graphene-like systems. Since the long-wave flexural mode has the lowest frequency, it is the easiest to excite [268]. At slightly higher frequencies, the out-of-plane ZO' mode (Fig. 7.6) can be observed, which corresponds to interlayer motion along the Z-axis (a layer-breathing mode). The other out-of-plane optic modes are characterised by the doubly degenerate $\mathrm{ZO}$ branch. At the $\Gamma$-point, the interlayer coupling causes the LO and TO modes to split into two doubly-degenerate branches, both of which correspond to in-plane relative motion of atoms. With the exception of the ZA and ZO' modes, all the frequency branches have symmetryimposed degeneracy at $\Gamma$. For the AA system, a small phonon instability is observed at the $\Gamma$-point, which is denoted by an imaginary mode $(\nu=\imath 1.04$ $\mathrm{THz}$ ). This indicates that the AA-system is dynamically unstable, and prefers to adopt the AB-stacking configuration, in accordance with the free energies of Fig. 7.5. As expected, the imaginary mode is a TA branch, which corresponds to the shear displacement of the layers with respect to one another. The ZA mode also shows instabilities in the vicinity of the zone-center, but has zero frequency at $\Gamma$. The ZO' breathing mode of AA-stacked bilayer graphene is located in a similar frequency range to the corresponding mode in $\mathrm{AB}$ graphene, at $\nu=2.16$ and $\nu=2.25 \mathrm{THz}$, respectively. The biggest frequency differences are observed for the TO modes, which in the $\mathrm{AB}$ system occurs at higher frequency than in the AA configuration, with $0.72 \mathrm{THz}$ of difference. This is partly because the $\mathrm{LO} / \mathrm{TO}$ is larger in the AA than the $\mathrm{AB}$ system $(0.57$ and $0.18 \mathrm{THz}$, respectively). Table 7.1 presents a summary of the zone-centre frequencies for the two stacking configurations. We note that the AA phonon dispersion does not correspond to that in Ref. [269], where, in contrast to the present results, imaginary frequencies are not observed (with calculations carried out using the Born-von-Karman model of lattice dynamics for in-plane atomic coupling and the Lennard-Jones potential for interlayer coupling [269]). 
Table 7.1: Frequencies $(\mathrm{THz})$ of the $\Gamma$-point phonon modes in $\mathrm{AB}$ - and AA-stacked bilayer graphene.

\begin{tabular}{cccccccc}
\hline Mode & ZA & ZO' & TA & LA & ZO & TO & LO \\
\hline AB & 0.00 & 2.25 & 0.82 & 0.00 & 26.72 & 47.86 & 48.04 \\
AA & 0.00 & 2.16 & $\imath 1.04$ & 0.00 & 26.82 & 47.14 & 47.71 \\
\hline
\end{tabular}

The branches which originate from the out-of-plane modes at the $\Gamma$-point, i.e. ZA, ZO' and ZO, become degenerate at the K-point (Fig. 7.6). The in-plane $\mathrm{LO}$ and LA phonon branches also meet at the K-point, giving rise to a doublydegenerate phonon band. It is also noteworthy that the dispersions of the out-of-plane modes behave linearly around the K-point in AA-BLG, whereas those in AB-BLG show a parabolic-like dispersion similar to that suggested in [269]. Features in the electronic spectra near the K-point in the two BLG systems are therefore also reflected in the phonon spectra (c.f. Figs. 7.2 and 7.6). Further lattice-dynamics calculations were carried out to investigate the effect of electric fields on the phonon dispersions (Fig. 7.7). Non-analytical corrections to the dynamical matrix at $\mathrm{q} \rightarrow 0$ were considered in all calculations. We find that the dispersion of the AA system is relatively insensitive to the applied external bias, and that for all applied fields the $\Gamma$-point instability persists. In comparison, the low-frequency branches of the $\mathrm{AB}$ band-structure show a significant response to the field (Figure 7.7). This effect results from the inclusion of non-analytical corrections; when these corrections are not included, the dispersions are relatively unaffected by the bias. The layer-breathing mode (ZO') displays a discontinuity at the $\Gamma$-point, with different frequencies for different directions of approach. Moreover, the flexural-acoustic (ZA) mode shows instabilities in the vicinity of the zone-centre, but continue to show zero frequency at the $\Gamma$-point. Since the long-wave flexural mode has the lowest frequency, it is the easiest to excite [268] and is therefore more sensitive to the bias. At the K-point (Figure 7.7, inset), as occurs for the electronic band-structure the degeneracy of the out-of-plane modes split, with the magnitude of the splitting depending on the size of the applied bias. 

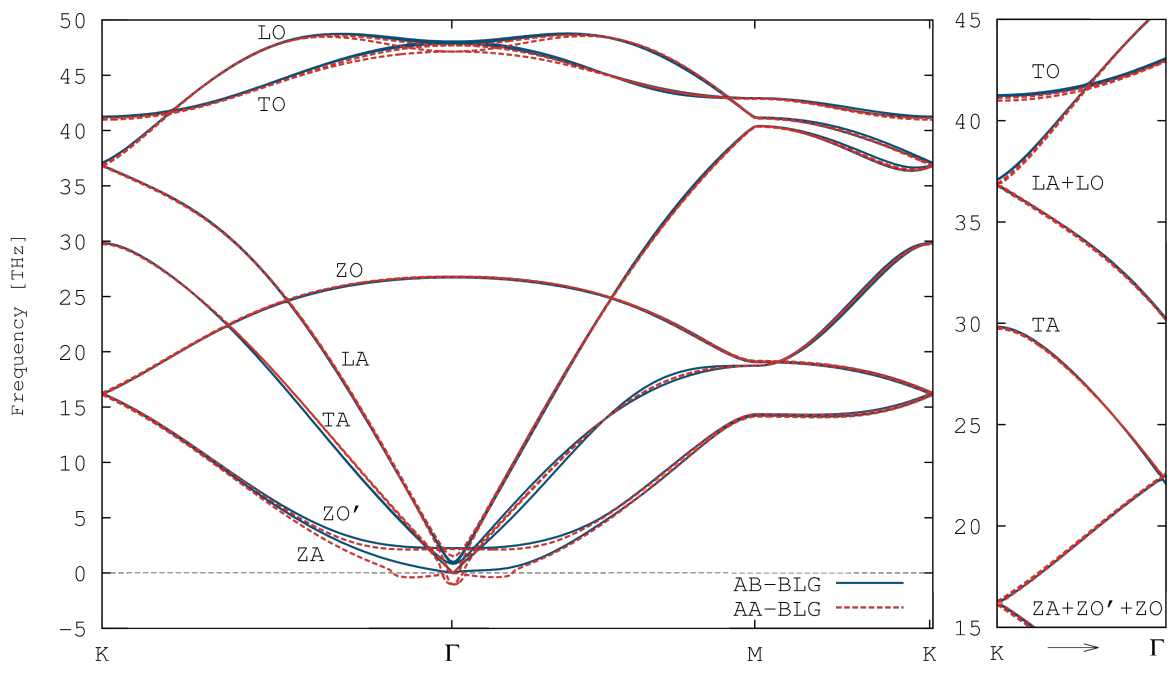

Figure 7.6: Phonon dispersions of the AB- and AA-stacked bilayer graphene systems computed with the harmonic approximation (blue solid and red dashed lines, respectively). The right-hand panel shows the dispersion along the K- $\Gamma$ path. The phonon branches are denoted by the symbols of the $\Gamma$-point phonons, several of which become degenerate at the $\mathrm{K}$-point. 

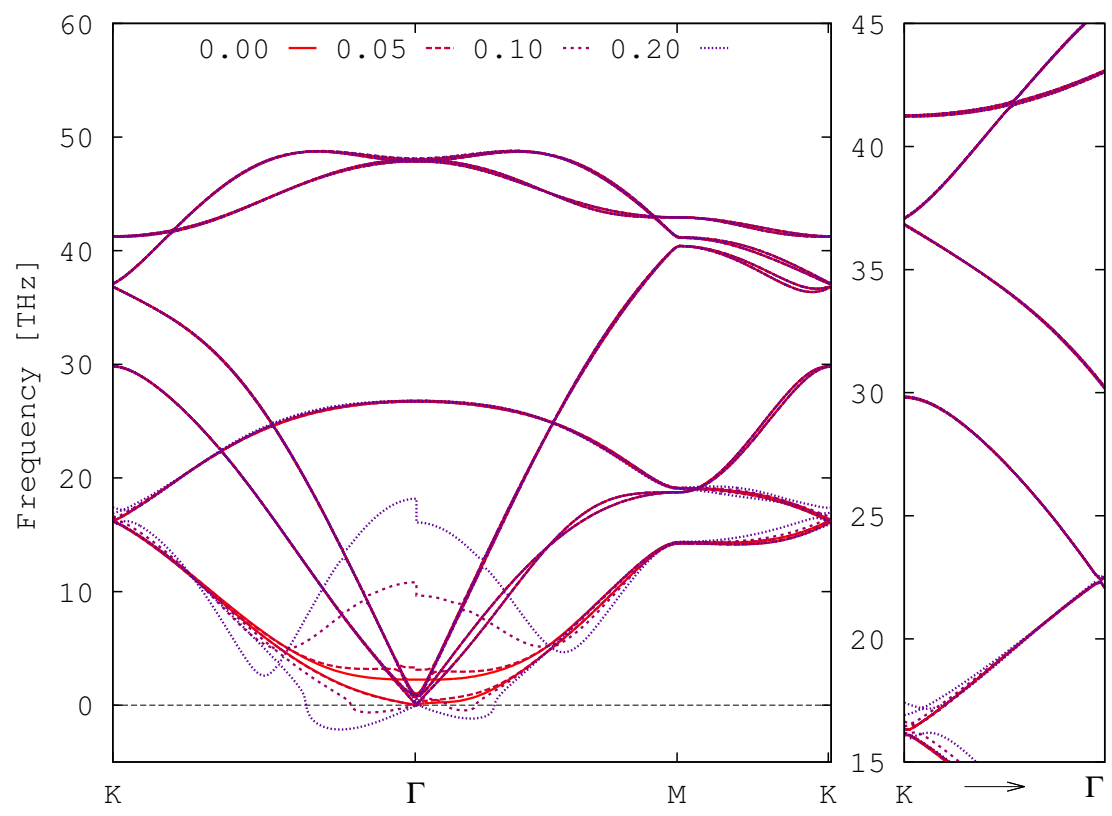

Figure 7.7: Harmonic phonon dispersions of the AB-stacking configurations of bilayer graphene under different applied bias. The right-hand panel in (a) shows the dispersion along the K- $\Gamma$ segment. The AA system shows doubly-degenerate imaginary modes at the zone-center, which is consistent with the alternative $\mathrm{AB}$ stacking being the most favourable arrangement. Non-analytical corrections have been applied to the dispersions of both systems. The ZA and ZO' modes on the AB-BLG change significantly under bias, whereas the applied field has comparatively little effect on the dispersion of the AA-BLG system. Electric fields are given in $\mathrm{eV} / \AA$. 


\section{Conclusions}

In summary, we have performed a detailed first-principles study of the effect of applied fields on the electronic structure and lattice dynamics of bilayer graphene. Application of an external field to the AB-stacked bilayer graphene system leads to drastic changes in the electronic properties, leading to the opening of the gap and asymmetry in the dispersion. This in turn induces in-plane inhomogeneities in the charge distribution on the sublattices, and the Coulomb interaction between electrons will thus cause a potential difference between the layers. Our results therefore show that the electron density can be controlled by tuning the band-gap width and dispersion asymmetry. Spin-orbit coupling has a significant effect on the dispersion as short-range electron-electron correlations become important. The Mexican-hat structure disappears under low bias, and the energy gap decreases. At larger field strength, the asymmetry in the dispersion persists, since the energy scale set by the Fermi-surface instability is minimized. On the other hand, the electronic structure of the AA system is relatively stable under bias. As for its electronic structure, applied fields cause the phonon dispersions of the AB-stacked system to change significantly when non-analytical corrections for long-range Coulomb interactions are taken into account. These corrections mainly affect the lower-frequency out-of-plane ZA and ZO' modes. The phonon dispersion of the AA system shows degenerate imaginary modes at the point, indicating the presence of a phonon instability. The dispersion of this stacking configuration is relatively insensitive to bias and does not change significantly in response to an applied field. In order to obtain better consistency with available literature, we would need to go beyond LDA functional. The ground-state is likely to have additional broken-symmetry configurations, and the lifting of spin and valley degeneracies may depend on long-range fluctuations, effects which are not well captured by local DFT functionals. For example, in the literature it has been observed that the AA stacking configuration may be stabilised by an excitonic gap [246]. To study such effects, one would need to resort to the two-body Green's function method (Bethe-Salpeter equation), a possibility which we are currently exploring. 


\section{Conclusions and General Discussion of the Thesis Results}

\subsection{High Critical Temperature Superconductivity}

The first three articles presented in this thesis (chapters three, four and five) are based mainly on the study of the electronic, optical, and transport properties when introducing disordered impurity centers into specific layered crystalline structures with the goal to study the high critical temperature superconductors. Moreover, we have seen that introducing such type of perturbations may change the electronic and optical properties by including a number of features that enrich our understanding of this general field in condensed matter physics.

In the first article we study the quasiparticle spectrum of $d$-wave superconductors with finite concentration of impurity centers. We demonstrated that the restructuring of the spectrum shows similarities to that previously established for point-like impurity perturbations; although some unique features of the extended nature of the perturbation also emerge. We found that the effects on the quasiparticle DOS and on the SC order parameter result from different irreducible representations of the point symmetry group of the impurity center. Compared to the case of spin-dependent extended perturbation, the 
suppression of SC order close to the considered impurity is much weaker. A generalization of the method of GEs for quasiparticle self-energy is obtained for the extended impurity centers. The self-consistent procedure is developed, generalizing the known SCTMA formulation for point-like centers, and a qualitative similarity with that case is demonstrated.

The second article emphasis the formation of impurity localized levels within the SC gap of SC iron pnictides, by considering local and nonmagnetic impurities. By increasing the impurity concentration, we show that these levels develop towards specific bands of extended quasiparticle states.By employing different types of GEs for a self-energy matrix, we show that the different energy states within the SC gap reveal a complex oscillatory structure of indirect interactions between impurity centers, showing crossovers between localized and extended states. The proposed theoretical methodology employed in this work can be further adapted for more involved types of impurity perturbations of SC iron pnictides; these include magnetic and nonlocal perturbations.

In the third article we study the effects of local and nonmagnetic impurities on the electronic transport properties of SC iron pnictides. Similarly to what had been considered in the second article, we observe a modification of the quasiparticle spectra in the doped compound - theses consist of the formation of localized in-gap impurity states and their development into specific narrow bands of impurity quasiparticles at impurity concentrations above a critical value, $c_{0}$. The main conclusions taken from this work shows that the transport properties of the studied systems are mainly affected by the impurity bandlike states that can be very strongly pronounced, either for high-frequency and for static transport processes. The experimental verification of the predictions taken from the presented work, would be of evident interest, since these can open perspectives for important practical applications, e.g., in narrow-band microwave devices or advanced low-temperature sensors.

Following the theoretical framework employed on the study of the electronic quasiparticle spectra, with the two-time Green's functions technique in presence of randomly distributed impurity centers, we can describe the physical parameters and use these for practical calculations of observable physical properties of the disordered systems.

The results obtained from the general framework of disordered systems consider the excitation spectra as divided into extended and localized states, with the existence of border points between states and known as mobility edges. Therefore, by changing the parameters of disorder (i.e. impurity concentration, impurity perturbation strength and symmetry) or by applying an exter- 
nal perturbation, it is possible to induce changes onto the parameters of the electronic spectrum.

For disordered systems, the Anderson model of disorder is usually applied. The Anderson transition considers that two mobility edges collapse at a certain critical strength of disorder (randomly fluctuating at each lattice site) so that the range of extended states disappears and in the spectrum only localized states emerge. Other types of similar electronic topological transitions can be considered when disorder by impurity perturbation is identical at randomly distributed sites. This is known as the Lifshitz model, and corresponds to the so-called coherent restructuring of spectrum when the impurity concentration reaches in average the level of overlap between the next-neighbor impurity states.

In conclusion, the distinction between two types of excitation states can be characterized by considering the above mentioned group expansions of Green's functions: the first which are the fully renormalized, and more adequate for extended states; or the second type, which are non-renormalized and best suited for localized states.

\subsection{Graphene-Based Systems}

With respect to the bilayer graphene system, we have shown the possibility of tuning the electrical conduction through variations of applied potential, by employing two different theoretical methodologies.

In the first study, we have considered the Green's function techniques adapted for a multiband electronic system and studied the effects of localized on-site perturbations by disperse impurities on a $\mathrm{AB}$-stacked bilayer graphene system under the applied electrical bias between the layers. The conditions for different types of localized impurity levels appearing within the bias-induced band gap and respective extension of these levels into specific narrow energy bands at impurity concentrations above certain characteristic values have been demonstrated. Some specifics of the studied system were shown to be considerably bias dependent of the impurity bands and of critical concentrations for their formation. These dependencies can be further analysed to provide some specific phase diagrams with respect to "bias concentrations", and similarly to what had been considered for antiferromagnetic insulators [132]. A practical application of the described electronic band structure is suggested in a form of highly sensitive bias control on the conductivity of the studied system through 
the impurity subband when brought close to a regime of bias-controlled Mott metal-insulator transition.

In the second study, we have considered application of an external field both to the AB- and AA-stacked bilayer graphene system by employing density functional theory. We have shown that such a perturbation on the AB system leads to drastic changes in the electronic properties, leading to the opening of the gap. This in turn induces in-plane inhomogeneities in the charge distribution on the sublattices, and the Coulomb interaction between electrons will thus cause a potential difference between the layers. Our results therefore show that the electron density can be controlled by tuning the band-gap width. As for the phonon properties of the $\mathrm{AB}$ system, applied fields cause the phonon dispersions to change significantly when non-analytical corrections for longrange Coulomb interactions are taken into account. These corrections mainly affect the lower-frequency out-of-plane ZA and ZO' modes. On the other hand, the electronic structure of the AA system is relatively stable under bias. The phonon dispersion of this latter system shows degenerate imaginary modes at the zone-centre, indicating the presence of a phonon instability. The dispersion of this stacking configuration is relatively insensitive to bias and does not change significantly in response to an applied field.

\subsection{Future Perspectives}

The Green's function analysis of the quasiparticle spectra in a SC iron pnictide with local and nonmagnetic impurities (simplest perturbation type) allows one to describe the formation of impurity localized levels within SC gap and, with increasing impurity concentration, the evolution of the localized states into bands of extended quasiparticle states. The developed spectral characteristics are used as a prediction for several observable impurity effects. The theoretical treatment employed in these works can be further adapted for analysis of more involved types of impurity perturbations of SC iron pnictides; these include magnetic and nonlocal perturbations. Moreover, work can be extended by considering the higher order terms of group expansions, which may allow one to obtain a more complete picture of different symmetry types of impurity effects on the SC material.

The considered theoretical model, used in the presented work, can also be broadened to include a more realistic multiorbital structures of the initial iron pnictide system and more general types of impurity perturbations, as already considered earlier for the $d$-wave cuprate systems [199]. Such application for 
iron pnictides can lead to some quantitative modifications of the results; however the main qualitative features regarding the possibility of forming new narrow in-gap quasiparticle bands and related sharp resonant peaks in transport coefficients should be still be evidneced.

The work carried so far on graphene was to consider monolayer or bilayer systems for which the sheets have been considered to be a 2D flatland. However, and taking into account that the monolayer graphene sheet is not a simple plane; in reality the sheet suffers certain ripples and curvatures, forming topological defects described by lattice deslocations. The Dirac cones of graphene can be deformed in different forms, depending on the way that deformations may modify the lattice. Strains, curvature, and topological defects influence the electronic states near the Dirac cones. Therefore, we intend to pursue this problematic by employing the well-known methods of Quantum Field Theory in Curved Space (QFTC). This method is well adapted to the study of the monolayer sheet under the influence of geometric deformations, since the electronic dynamics is already described to be of relativistic-type. In curved space one needs to work the problem with the level of QFTC.

Within the framework of the quantum field theory in curved space, and by adopting a low-energy effective Dirac equation by considering a curved space metric, we expect to obtain a geometry-induced field to describe the graphene deformation. We envisage that from a specific out-of-plane deformation, which satisfies the buckled honeycomb structure, it is possible to arrive at the Dirac harmonic oscillator equation, where the pseudo-magnetic field naturally appears. As a consequence of the Dirac harmonic oscillator, it is possible to address the physics of the Landau levels. Through mechanical strain, induced on the graphene lattice structure it is possible to tune the dynamics of the charge carriers. The electro-mechanical coupling yields very large pseudo-magnetic fields for small strain fields, which offer new scientific opportunities which are not possible to perform with ordinary laboratory magnets.

The study of geometric deformation on graphene had been studied recently in works carried out by Chaves et al.[94]. However, we intend to pursue a distinct geometric deformation considering a more natural curvature that satisfies the lattice symmetry of the system. Moreover, such a curvature is a generalization of the works presented in the theoretical framework in subsection 2.6.4, which we envisage a future manuscript for publication.

The geometric language to describe electronic properties of graphene will be explored. In-plane and out-of-the plane displacements of graphene are expected to be formulated as curvature and the corresponding Dirac equation 
derived for a general geometry. A simple periodic out-of-the plane ripple is to be formulated and investigated, and we expect that the ripple will induce a mass gap on the electronic a band structure, due to breaking of the sublattice symmetry of the graphene sheet.

Perturbations to the system (deformation, defects, disorder) are usually studied by means of Tight-Binding methodologies; or through continuum models by employing quantum field theory in curved spacetime. Currently atomistic computational methodologies applied to solid state physics or chemistry, come as an alternative since these are quite robust and efficient to compute several observables. Therefore, another future research perspective will be to perform ab-initio density functional theory calculations on different deformed graphene sheets with different applied strain fields, where the deformation amplitude and modulation frequency (lattice parameter; supercell size) should satisfy the symmetry of the buckled honeycomb structure.

The hexagonal unit-cell of the pristine single-sheet graphene is composed by two atoms. However such a small unit-cell is not sufficient to simulate the deformation potential, and such has to be modelled by the use of supercells. However heavier computational resources are required to perform the desired calculations. Therefore one has to find a compromise between the size of the system and the available resources.

Another prospect that we intend to pursue as continuation of the work carried out on the AB- and AA-BLG systems under applied electric fields, is to compute the lattice thermal conductivity $(\kappa)$ for each system, by employing thirdorder phonon-phonon interactions. We expect that by increasing the external bias, $\kappa$ will decrease. Since the electric field induces the opening of an electronic band gap for the AB system, evidencing a semimetal-to-semiconductor phase transition, this effect will thus affect $\kappa$. On the other hand, the lattice thermal conductivity will tend to decrease due to disorder introduced in the system thus altering the scattering rate of the phonon-phonon collisions.

Graphene is a very promising material for electronic devices. Moreover from straintronics, it is possible to engineer the electronic and optical properties to ensure that the system can be employed for the desired applications. For example, bilayer graphene system which by applying external bias (chapter 5 and 6) one can modify the electronic band gap. 


\section{Bibliography}

[1] H. Kamerlingh-Onnes. The Superconductivity of Mercury. Comm. Phys. Lab. Univ. Leiden, 122, 1911.

[2] G.F. Hardy and J.K. Hulm. Superconducting Silicides and Germanides. Phys. Rev., 89:884, 1953.

[3] G.F. Hardy and J.K. Hulm. The Superconductivity of Some Transition Metal Compounds. Phys. Rev., 93:1004, 1954.

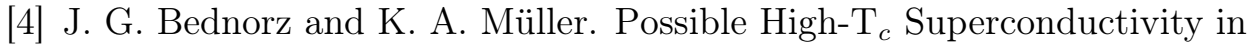
the Ba-La-Cu-O System. Z. Phys. B, 64:189, 1986.

[5] M. K. Wu, J. R. Ashburn, C. J. Torng, P. H. Hor, R. L. Meng, L. Gao, Z. J. Huang, Y. Q. Wang, and C. W. Chu. Superconductivity at $93 \mathrm{~K}$ in a New Mixed-Phase Y-Ba-Cu-O Compound System at Ambient Pressure. Phys. Rev. Lett., 58:908, 1987.

[6] R. L. Meng L. Gao Z. J. Huang C. W. Chu, P. H. Hor and Y. Q. Wang. Evidence for superconductivity Above $40 \mathrm{~K}$ in the La-Ba-Cu-O Compound System. Phys. Rev. Lett., 58:405, 1987.

[7] K. S. Novoselov, A. K. Geim, S. V. Morozov, D. Jaing, Y. Zhang, S. V. Dubonos, I. V. Grigorieva, and A. A. Firsov. Electric Field Effect in Atomically Thin Carbon Films. Science, 306:666, 2004.

[8] P. R. Wallace. The Band Theory of Graphite. Phys. Rev., 77:622, 1947. 
[9] G. Semenoff. Condensed-Matter Simulation of a Three-Dimensional Anomaly. Phys. Rev. Lett., 53:2449, 1984.

[10] K. S. Novoselov, E. McCann, S. V. Morozov, V. I. Fal'ko, M. I. Katsnelson, U. Zeitler, D. Jiang, F. Schedin, and A. K. Geim. Unconventional Quantum Hall Effect and Berry's Phase of $2 \pi$ in Bilayer Graphene. Nat. Phys., 2:177, 2006.

[11] Y. Araki and T. Hatsuda. Chiral Gap and Collective excitations in Monolayer Graphene from Strong Coupling Expansion of Lattice Gauge Theory. Phys. Rev. B, 82:121403(R), 2010.

[12] V. P. Gusynin and S. G. Sharapov. Unconventional Integer Quantum Hall Effect in Graphene. Phys. Rev. Lett., 95:146801, 2005.

[13] J.P. Carbotte V.P. Gusynin, S.G. Sharapov. AC Conductivity of Graphene: from tight-binding Model to 2+1-dimensional Quantum Electrodynamics. Int.J.Mod.Phys., 21:4611, 2007.

[14] D. V. Khveshchenko. Ghost Excitonic Insulator Transition in Layered Graphite. Phys. Rev. Lett., 87:246802, 2001.

[15] D. V. Khveshchenko. Magnetic-Field-Induced Insulating Behavior in Highly Oriented Pyrolitic Graphite. Phys. Rev. Lett., 87:206401, 2001.

[16] D. V. Khveshchenko. Electron Localization Properties in Graphene. Phys. Rev. Lett., 97:036802, 2006.

[17] D. V. Khveschenko, A. G. Yashenkin, and I. V. Gornyj. Interacting Random Dirac Fermions in Superconducting Cuprates. Phys. Rev. Lett., 86:4668, 2001.

[18] V. P. Gusynin, V. A. Miransky, and I. A. Shovkovy. Catalysis of Dynamical Flavor Symmetry Breaking by a Magnetic Field in $2+1$ Dimensions. Phys. Rev. Lett., 73:3499, 1994.

[19] Y. Kopelevich, P. Esquinazi, J. H. S. Torres, and S. Moehlecke. Ferromagnetic- and superconducting-like behavior of graphite. J. Low Temp. Phys., 119:691, 2000.

[20] Y. Araki. Chiral Symmetry Restoration in Monolayer Graphene Induced by Kekule Distortion. Phys. Rev. B, 84:113402, 2011.

[21] C. Berger, Z. Song, T. Li, X. Li, A. Y. Ogbazghi, R. Feng, Z. Dai, A. N. Marchenkov, E. H. Conrad, P. N. First, and W. A. de Heer. Ultrathin Epitaxial Graphite: 2D Electron Gas Properties and a Route Toward Graphene-Based Nanoelectronics. J. Phys. Chem., 108:19912, 2004. 
[22] Y. G. Pogorelov, M. C. Santos, and V. M. Loktev. Strongly Correlated Systems, Coherence and Entanglement, page 443. World Scientific, 2007.

[23] Y. G. Pogorelov, M. C. Santos, and V. M. Loktev. Specifics of Impurity Effects in Ferropnictide Superconductors. Phys. Rev. B, 84:144510, 2011.

[24] J. M. Ziman. Models of Disorder the Theoretical Physics of Homogeneously Disordered Systems. Cambridge University Press, 1979.

[25] C. Kittel. Introduction to Solid State Physics. Wiley, 1986.

[26] C. Kittel. Quantum Theory of Solids. Wiley, 1987.

[27] M. P. Marder. Condensed Matter Physics (chapter 9). A WileyInterscience Publication, 2000.

[28] N. W. Ashcroft and N. D. Mermin. Solid State Physics. Thomson Learning Inc., 1976.

[29] G. Grosso and G. P. Parravicini. Solid State Physics. Elsevier Ltd., 2014.

[30] H. Kleinert. Gauge fields in condensed matter. World Scientific, 1, 1987.

[31] J. Robertson. High Dielectric Constant Gate Oxides for Metal Oxide Si Transistors. Rep. Prog. Phys., 69:332, 2006.

[32] C. G. Van de Walle and J. Neugebauer. Universal Alignment of Hydrogen Levels in Semiconductors, Insulators and Solutions. Nature (London), 423:626, 2003.

[33] E. L. Silva, A. G. Marinopoulos, R. C. Vilão, R. B. L. Vieira, H. V. Alberto, J. Piroto Duarte, and J. M. Gil. Hydrogen Impurity in Yttria: Ab-Initio and $\mu$ SR Perspectives. Phys. Rev. B, 85:165211, 2012.

[34] B. I. Shklovskii and A. L. Efros. Electronic Properties of Doped Semiconductors. Berlin: Springer-Verlag, 1984.

[35] R. Enderlein. Photoreflectance studies of (Al, Ga)As/GaAs heterostructures and devices. Phys. Stat. Sol. (b), 194:257, 1996.

[36] R. Enderlein and N.J.M. Horing. Fundamentals of Semiconductor Physics and Devices. World Scientific Pub Co Inc, 1997.

[37] S.M. Sze and K. K. Ng. J. Physics of Semiconductor Devices. 2006.

[38] I. Tamm. On the Possible Bound States of Electrons on a Crystal Surface. Phys. Z. Soviet Union, 1:733, 1932. 
[39] M. Steslicka S. G. Davison. Basic Theory of Surface States. Oxford Univ. Pr., 1992.

[40] W. Shockley. On the surface states associated with a periodic potential. Phys. Rev., 56:317, 1939.

[41] R. Dalven. Introduction to Applied Solid State Physics. New York, Plenum Press., 1990.

[42] A. Many, Y. Goldstein, and N.B. Grover. Semiconductor Surfaces. North Holland Publishing Company-Amsterdam, 1965.

[43] Bardeen. Surface states and rectification at a metal semi-conductor contact. J. Phys. Rev., 71:717, 1947.

[44] J.S. Hwang, W.Y. Chou am S.L. Tyan, H.H. Lin, and T.L. Lee. Study of surface Fermi level and surface state distribution in InAlAs surfaceintrinsic- $n^{+}$structure by photoreflectance. Appl. Phys. Lett., 67:2350, 1995.

[45] J.S. Hwang, W.Y. Chau, M.C. Hung, J.S. Wang, and H.H. Lin. Built-in electric field and surface Fermi level in InP surface-intrinsic $n^{+}$structures by modulation spectroscopy. J. Appl. Phys., 82:3888, 1997.

[46] R. Enderlein, D. Beliaev, J. A. N. T. Soares, L. M. R. Scolfaro, and J. R. Leite. Method for calculating photo- and electroreflectance spectra from semiconductor heterostructures. Phys. Rev. B, 52:2814, 1995.

[47] N.F. Mott. Metal-Insulator Transitions. 1974.

[48] P.W. Anderson. Absence of Diffusion in Certain Random Lattices. Phys. Rev., 109:1492, 1958.

[49] T.V. Ramakrishnan P.A. Lee. Disordered Electronic Systems. Rev. Mod. Phys., 57:287, 1985.

[50] C. Di Castro. In Anderson Localization, volume 28. Springer Proc. in Physics, eds. Ando, T. and Fukuyama, H., 1988.

[51] B.L. Altshuler, A.G. Aronov, D.E. Khmelnitskii, and A.I. Larkin. Coherent Effects in Disordered Conductores in Quantum Theory of Solids. Quantum Theory of Solids, ed. I.M. Lifshits; MIR, Moscow, 1982.

[52] N.F. Mott and Davis. Electronic Processes in Non-crystalline Materials. Clarendon, Oxford, 1971. 
[53] A.F. Ioffe and A.R. Regel. Non-Crystalline, Amorphous and Liquid Electronic Semiconductors. Prog. Semicond., 4:237, 1960.

[54] D.J. Thouless. Maximum metallic resistance in thin wires. Phys. Rev. Lett., 39:1167, 1977.

[55] E. Abrahams, P.W. Anderson, D.C. Licciardello, and T.V. Ramakrishnan. Scaling theory of localization: Absence of quantum diffusion in two dimensions. Phys. Rev. Lett., 42:673, 1979.

[56] N. N. Bogolyubov and S. V. Tyablikov. Retarded and Advanced Green Functions in Statistical Physics. Sov. Phys. Dokl, 4:589, 1959.

[57] W. Kohn and L. J. Sham. Self-Consistent Equations Including Exchange and Correlation Effects. Phys. Rev., 140:A1133, 1965.

[58] P. Hohenberg and W. Kohn. Phys. Rev., 136:B864, 1964.

[59] F. Nogueira, A. Castro, and M. A. L. Marques. A Primer in Density Functional Theory, chapter 6, page 218. Springer, 2002.

[60] R. G. Parr and W. Yang. Density-Functional Theory of Atoms and Molecules. Oxford: Oxford University Press, 1994.

[61] W. Koch and M. C. Holthausen. A Chemist's Guide to Density Functional Theory. Wiley-VCH Verlag GmbH, second edition edition, 2001.

[62] E. Fermi. Il Nuovo Cimento, 11:157, 1934.

[63] H. Hellmann. Journal of Chemical Physics, 3:61, 1935.

[64] J. C. Phillips and L. Kleinman. New Method for Calculating Wave Functions in Crystals and Molecules. Phys. Rev., 116:287, 1959.

[65] D. R. Hamann, M. Schlüter, and C. Chiang. Norm-Conserving Pseudopotentials. Phys. Rev. Lett., 43:1494, 1979.

[66] R. M. Martin. Electronic Structure - Basic Theory and Practical Methods. Cambridge University Press, 2004.

[67] Wikimedia Commons. Wikimedia commons.

[68] D. Vanderbilt. Soft Self-Consistent Pseudopotentials in a Generalized Eigenvalue Formalism. Phys. Rev. B, 41:7892(R), 1990.

[69] P. E. Blöchl. Projector Augmented-Wave Method. Phys. Rev. B, 50:17953, 1994. 
[70] G. Kresse and D. Joubert. From Ultrasoft Pseudopotentials to the Projector Augmented-Wave Method. Phys. Rev. B, 59:1758, 1999.

[71] D. M. Ceperley and B. J. Alder. Phys. Rev. Lett., 45:566, 1980.

[72] J. P. Perdew and A. Zunger. Self-Interaction Correction to DensityFunctional Approximations for Many-Electron Systems. Phys. Rev. B, 23:5048, 1981.

[73] J. P. Perdew and Y. Wang. Accurate and Simple Analytic Representation of the Electron-Gas Correlation Energy. Phys. Rev. B, 45:13244, 1992.

[74] H. Jiang, R. I. Gomez-Abal, P. Rinke, and M. Scheffler. Localized and Itinerant States in Lanthanide Oxides United by $G W @ L D A+U$. Phys. Rev. Lett., 102:126403, 2009.

[75] J. P. Perdew, A. Ruzsinszky, G. I. Csonka, O. A. Vydrov, G. E. Scuseria, L. A. Constantin, X. Zhou, and K. Burke. Restoring the densitygradient expansion for exchange in solids and surfaces. Phys. Rev. Lett., 100:136406, 2008.

[76] J. P. Perdew, A. Ruzsinszky, G. I. Csonka, O. A. Vydrov, G. E. Scuseria, L. A. Constantin, X. Zhou, and Kieron Burke. Erratum: Restoring the Density-Gradient Expansion for Exchange in Solids and Surfaces [Phys. Rev. Lett. 100, 136406 (2008)]. Phys. Rev. Lett., 102:039902, 2009.

[77] J. P. Perdew and S. Kurth. A Primer in Density Functional Theory, chapter 1, page 1. Springer, 2002.

[78] M. T. Dove. Structure and Dynamics, chapter 12. Oxford University Press, 2003.

[79] M. T. Dove. Introduction to Lattice Dynamics, chapter 8. Cambridge University Press, 1993.

[80] L. Paulatto, I. Errea, M. Calandra, and F. Mauri. First-Principles Calculations of Phonon Frequencies, Lifetimes, and Spectral Functions from Weak to Strong Anharmonicity: The Example of Palladium Hydrides. Phys. Rev. B, 91:054304, 2015.

[81] K. Parlinski, Z. Q. Li, and Y. Kawazoe. First-Principles Determination of the Soft Mode in Cubic $\mathrm{ZrO}_{2}$. Phys. Rev. Lett., 78:4063, 1997.

[82] L. Chaput, A. Togo, I. Tanaka, and G. Hug. Phonon-Phonon Interactions in Transition Metals. Phys. Rev. B, 84:094302, 2001. 
[83] J. M. Skelton, S. C. Parker, A. Togo, I. Tanaka, and A. Walsh. Thermal Physics of the Lead Chalcogenides PbS, PbSe, and PbTe from First Principles. Phys. Rev. B, 89:205203, 2014.

[84] L.Schwartz. Theory of Distributions. Hermann, 1950.

[85] J. E. Mayer and M. Goeppert-Mayer. Statistical mechanics. Wiley, 1940.

[86] C. J. Pethick and D. Pines. Transport Processes in Heavy-Fermion Superconductors. Phys. Rev. Lett., 57:118, 1986.

[87] Y. Pogorelov. Ground State Symmetry and Impurity Effects in Superconductors. Solid State Commun., 95:245, 1995.

[88] I. M. Lifshitz. Energy Spectrum of Disordered Systems. Adv. Phys., 13:483, 1964.

[89] A. H. Castro Neto, F. Guinea, N. M. R. Peres, K. S. Novoselov, and A. K. Geim. The Electronic Properties of Graphene. Rev. Mod. Phys., 81:109, 2009.

[90] V.A.Fock. Princípios de Mecânica Quântica. Mir, 1986.

[91] Yu.V. Skrypnyk and V.M. Loktev. Impurity Effects in a TwoDimensional System with the Dirac Spectrum. Phys. Rev. B., 73:241402(R), 2006.

[92] I. M. Lifshitz, S.A. Gredeskul, and L.A. Pastur. Introduction to the Theory of Disordered Systems. Wiley and Sons, New York, 1988.

[93] S.S. Pershoguba, Yu.V. Skrypnyk, and V.M. Loktev. Numerical Evidence of Spectrum Rearranjement in Impure Graphene. Phys. Rev. B, 80:214201, 2009.

[94] A. J. Chaves, T. Frederico, O. Oliveira, W. de Paula, and M. C. Santos. Optical Conductivity of Curved Graphene. J. Phys. Condens. Matter., 26:185301, 2014.

[95] N. Levy, S. A. Burke, K. L. Meaker, M. Panlasigui, A. Zettl, F. Guinea, A. H. C. Neto, and M. F. Crommie. Strain-Induced Pseudo-Magnetic Fields Greater than 300 Tesla in Graphene Nanobubbles. Science, 329:544, 2010.

[96] F. de Juan, A. Cortijo, M. A. H. Vozmediano, and A. Cano. Aharonov-Bohm Interferences from Local Deformations in Graphene. Nat. Phys., 7:810, 2011. 
[97] S. Zhu, J. A. Stroscio, and T. Li. Programmable Extreme Pseudomagnetic Fields in Graphene by a Uniaxial Stretch. Phys. Rev. Lett., 115:245501, 2015.

[98] C. Kane and E. Mele. Size, Shape, and Low Energy Electronic Structure of Carbon Nanotubes. Phys. Rev. Lett., 78:1932, 1997.

[99] M. Katsnelson and K. Novoselov. Graphene: New Bridge between Condensed Matter Physics and Quantum Electrodynamics. Solid State Commun., 143:3, 2007.

[100] F. Guinea, M. Katsnelson, and M. Vozmediano. Midgap States and Charge Inhomogeneities in Corrugated Graphene. Phys. Rev. B, 77:075422, 2008.

[101] F. Guinea, M. I. Katsnelson, and A. K. Geim. Energy Gaps and a ZeroHeld Quantum Hall Effect in Graphene by Strain Engineering. Nat. Phys., 6:30, 2009.

[102] F. Guinea, A. K. Geim, M. I. Katsnelson, and K. S. Novoselov. Generating Quantizing Pseudomagnetic Fields by Bending Graphene Ribbons. Phys. Rev. B, 81:035408, 2010.

[103] I. Y. Sahalianov, T. M. Radchenko, V. A. Tatarenko, G. Cuniberti, and Y. I. Prylutskyy. Straintronics in Graphene: Extra Large Electronic Band Gap Induced by Tensile and Shear Strains. J. App. Phys., 126:054302, 2019.

[104] M. Cariglia, R. Giambò, and A. Perali. Electronic Properties of Curved Few-Layers Graphene: A Geometrical Approach. Condensed Matter, $3: 11,2018$.

[105] D. Itô, K. Mori, and E. Carriere. An Example of Dynamical Systems with Linear Trajectory. Il Nuovo Cimento, 51:1119, 1967.

[106] B. P. Mandal and S. Verma. Dirac Oscillator in an External Magnetic Field. Phys. Lett. A, 374:1021, 2010.

[107] C. Quimbay and P. Strange. Graphene Physics via the Dirac Oscillator in $(2+1)$. arXiv:1311.2021/cond-mat.mes-hall], 2013.

[108] A. Bermudez, M. A. Martin-Delgado, and E. Solano. Exact Mapping of the $2+1$ Dirac Oscillator onto the Jaynes-Cummings Model: Ion-Trap Experimental Proposal. Phys. Rev. A, 76:041801, 2007. 
[109] P. Rozmej and R. Arvieu. The Dirac Oscillator. A Relativistic version of the Jaynes-Cummings Model. J. Phys. A, 32:5367, 1999.

[110] E. Arias, A. R. Hernández, and C. Lewenkopf. Gauge Fields in Graphene with Nonuniform Elastic Deformations: A Quantum Field Theory Approach. Phys. Rev. B, 92:245110, 2015.

[111] D. M. Ginsberg. Physical Properties of High Temperature Superconductors III. World Scientific, Singapore, 1992.

[112] C. C. Tsuei and J.R. Kirtley. Pairing Symmetry in Cuprate Superconductors. Rev. Mod. Phys., 72:969, 2000.

[113] P. W. Anderson. Theory of Dirty Superconductors. J. Phys. Chem. Solids, 11:26, 1959.

[114] V.M. Loktev and Yu.G. Pogorelov. Formation of d-wave Superconducting order in a Randomly Doped Lattice. Low Temp. Phys., 27:1039, 2001.

[115] A. V. Balatsky, I. Vekhter, and J. X. Zhu. Impurity-Induced States in Conventional and Unconventional Superconductors. Rev. Mod. Phys., 78:373, 2006.

[116] V.M. Loktev and Yu.G. Pogorelov. Density of states in doped metal with d-wave superconducting order parameter. Europhys. Lett., 58:549, 2002.

[117] E. W. Hudson S.H. Pan, K. M. Lang, H. Eisaki, S. Ushida, and J. C. Davis. Imaging the Effects of Individual Zinc Atoms on Superconductivity in $\mathrm{Bi}_{2} \mathrm{Sr}_{2} \mathrm{CaCu}_{2} \mathrm{O}_{8+}$. Nature (London), 403:746, 2000.

[118] P. A. Lee. Localized States in a $d$-Wave Superconductor. Phys. Rev. Lett., 71:1887, 1993.

[119] M. Franz, C. Kallin, and A.J. Berlinsky. Impurity scattering and localization in d-wave superconductors. Phys. Rev. B, 54:R6897, 1996.

[120] W. A. Atkinson, P. J. Hirschfeld, and A. H. McDonald. Gap Inhomogeneity and the Density of States in Disordered $d$-Wave Superconductors. Phys. Rev. Lett., 85:3922, 2000.

[121] A. Polkovnikov, S. Sachdev, and M. Vojta. Impurity in a d-wave superconductor: Kondo effect and STM spectra. Phys. Rev. Lett., 86:296, 2001. 
[122] I. Adagideli, P.M. Goldbart, A. Shnirman, and A. Yazdani. Low-Energy Quasiparticle States near Extended Scatterers in d-Wave Superconductors and their Connection with SUSY Quantum Mechanics. Phys. Rev. Lett., 83:5571, 1999.

[123] A. V. Balatsky, M. I. Salkola, and A. Rozengren. Impurity-Induced Virtual Bound States in $d$-Wave Superconductors. Phys. Rev. B, 51:15547, 1995.

[124] V. M. Loktev and Yu. G. Pogorelov. Magnetic Effect from Non-Magnetic Impurity in Superconducting $\mathrm{CuO}_{2}$ plane. Europhys. Lett., 60:757, 2002.

[125] J. C. Campuzano, G. Jennings, M. Faiz, L. Beaulaigue, B. W. Veal, J. Z. Liu, A. P. Paulikas, K. Vandervoort, H. Claus, R. S. List, A. J. Arko, and R. J. Bartlett. Fermi surfaces of $\mathrm{yba}_{2} \mathrm{Cu}_{3} \mathrm{O}_{6.9}$ as seen by angle-resolved photoemission. Phys. Rev. Lett., 64:2308, 1990.

[126] Z.-X. Shen and D.S. Dessau. Electronic structure and photoemission studies of late transition-metal oxides - mott insulators and hightemperature superconductors. Phys. Rep., 253:1-162, 1995.

[127] H. Ding, M. R. Norman, T. Yokoya, T. Takeuchi, M. Randeria, J. C. Campuzano, T. Takahashi, T. Mochiku, and K. Kadowaki. Evolution of the fermi surface with carrier concentration in $\mathrm{bi}_{2} \mathrm{Sr}_{2} \mathrm{cacu}_{2} \mathrm{O}_{8+\delta}$. Phys. Rev. Lett., 78:2628, 1997.

[128] A. Ino, C. Kim, T. Mizokawa, Z.-X. Shen, A. Fujimori, M. Takaba, K. Tamasaku, H. Eisaki, and S. Uchida. Fermi surface and band dispersion in $\mathrm{La}_{2-x} \mathrm{Sr}_{x} \mathrm{CuO}_{4}$. J. Phys. Soc. Jpn., 68:1496, 1999.

[129] P.J. Hirshfeld, P. Wolfle, and D. Einzel. Consequences of Resonant Impurity Scattering in Anisotropic Superconductors: Thermal and Spin Relaxation Properties. Phys. Rev. B, 37:83, 1988.

[130] T. Xiang and J. M. Wheatley. Nonmagnetic Impurities in TwoDimensional Superconductors. Phys. Rev. B, 51:11721, 1995.

[131] F.A. Cotton. Chemical Applications of Group Theory. Wiley-NY, 1990.

[132] M. A. Ivanov, V. M. Loktev, and Yu. G. Pogorelov. Long-Range Impurity States in Magnetic Crystals. Phys. Rep., 153:209, 1987.

[133] Yu.A. Izyumov and M.V. Medvedev. Magnetically Ordered Crystals Containing Impurities. Plenium NY, 1973. 
[134] M. A. Ivanov. Dynamics of Quasilocalized Vibrations with a High Concentration of Impurity Centers. Sov. Phys. Solid State, 12:1508, 1971.

[135] V.M. Loktev and Yu.G. Pogorelov. Metallization and superconductivity in doped metal-oxide layered compounds. Physica C, 272:151, 1996.

[136] A. Perali, P. Pieri, and G.C. Strinati. Comment on: BCS to BoseEinstein Crossover Phase Diagram at Zero Temperature for a $\mathrm{d}_{\left(x^{2}-y^{2}\right)}$ Order Parameter Superconductor: Dependence on the Tight Binding Structure. Phys. Rev. B, 68:066501, 2003.

[137] V. M. Loktev and Yu. G. Pogorelov. Validity of Self-Consistent T-Matrix for Doped $d$-Wave Superconductors. Phys. Lett. A, 320:307, 2004.

[138] A. A. Abrikosov and L. P. Gor'kov. Contribution to the Theory of Superconducting Alloys with Paramagnetic Impurities. Sov. Phys. JETP, 12:1243, 1961.

[139] J. P. Perdew, K. Burke, and M. Ernzerhof. Self-Consistent Approximations in Many-Body Systems. Phys. Rev., 127:1391, 1962.

[140] Y. Kamihara, H. Hiramatsu, M. Hirano, R. Kawamura, H. Yanagi, T. Kamiya, and H. Hosono. Iron-Based Layered Superconductor: LaOFeP. J. Am. Chem. Soc., 128:10012, 2006.

[141] Y. Kamihara, T. Watanabe, M. Hirano, and H. Hosono. Iron-based Layered Superconductor $\mathrm{La}\left[\mathrm{O}_{1-x} \mathrm{~F}_{x}\right] \mathrm{FeAs}(\mathrm{x}=0.05-0.12)$ with $\mathrm{T}_{c}=26$ K. J. Am. Chem. Soc., 130:3296, 2008.

[142] M. V. Sadovskii. High-Temperature Superconductivity in Iron-Based Layered Iron Compounds. Phys. Usp., 51:1201, dec 2008.

[143] Yu. A. Izyumov and E. Z. Kurmaev. FeAs Systems: a New Class of High-Temperature Superconductors. Phys. Usp., 51:1261, 2008.

[144] D. M. Ginsberg. Physical Properties of High Temperature Superconductors I. World Scientific, Singapore, 1989.

[145] H. Takahashi, K. Igawa, K. Arii, Y. Kamihara, M. Hirano, and H. Hosono. Superconductivity at $43 \mathrm{k}$ in an Iron-Based Layered Compound $\mathrm{LaO}_{1-x} \mathrm{~F}_{x}$ FeAs. Nature, 453:376, 2008.

[146] M. R. Norman. High-Temperature Superconductivity in the Iron Pnictides. Physics, 1:21, 2008. 
[147] H. Ding, P. Richard, K. Nakayama, K. Sugawara, T. Arakane, Y. Sekiba, A. Takayama, S. Souma, T. Sato, T. Takahashi, Z. Wang, X. Dai, Z. Fang, G. F. Chen, J. L. Luo, and N. L. Wang. Observation of FermiSurface Dependent Nodeless Superconducting Gaps in $\mathrm{Ba}_{0} \cdot 6 \mathrm{~K}_{0} \cdot 4 \mathrm{Fe}_{2} \mathrm{As}_{2}$. Euro Phys. Lett., 83:47001, 2008.

[148] T. Kondo, A. F. S.-Syro, O. Copie, C. Liu, M. E. Tillman, E. D. Mun, J. Schmalian, S. L. Budko, M. A. Tanatar, P. C. Canfield, and A. Kaminski. Momentum Dependence of the Superconducting Gap in $\mathrm{NdFeAsO}_{0} .9 \mathrm{~F}_{0} .1$ Single Crystals Measured by Angle Resolved Photoemission Spectroscopy. Phys. Rev. Lett., 101:147003, 2008. and references therein.

[149] I. I. Mazin, D. J. Singh, M. D. Johannes, and M. H. Du. Unconventional Superconductivity with a Sign Reversal in the Order Parameter of LaFeAsO $_{1-x} \mathrm{~F}_{x}$. Phys. Rev. Lett., 101:057003, 2008.

[150] D. J. Singh and M.-H. Du. Density Functional Study of $\mathrm{LaFeAsO}_{1-x} \mathrm{~F}_{x}$ : a Low Carrier Density Superconductor near Itinerant Magnetism. Phys. Rev. Lett., 100:237003, 2008.

[151] K. Haule, J. H. Shim, and G. Kotliar. Correlated Electronic Structure of $\mathrm{LaO}_{1-x} \mathrm{~F}_{x} \mathrm{FeAs}$. Phys. Rev. Lett., 100:226402, 2008.

[152] G. Xu, W. Ming, Y. Yao, X. Dai, S.-C. Zhang, and Z. Fang. Europhys Lett., 82:67002, 2008.

[153] C. Cao, P. J. Hirschfeld, and H. P. Cheng. Proximity of antiferromagnetism and superconductivity in $\mathrm{LaFeAsO}_{1-x} \mathrm{~F}_{x}$ : effective hamiltonian from ab initio studies. Phys. Rev. B., 77:220506(R), 2008.

[154] S. Raghu, X.-L. Qi, C.-X. Liu, D.J. Scalapino, and S.-C. Zhang. Minimal Two-Band Model of the Superconducting Iron Oxypnictides. Phys. Rev. B, 77:220503(R), 2008.

[155] K. Kuroki, S. Onari, R. Arita, H. Usui, Y. Tanaka, H. Kontani, and H. Aoki. Unconventional Pairing Originating from the Disconnected Fermi Surfaces of Superconducting $\mathrm{LaFeAsO}_{1-x} \mathrm{~F}_{x}$. Phys. Rev. Lett., 101:087004, 2008.

[156] A. V. Chubukov, D. V. Efremov, and I. Eremin. Magnetism, Superconductivity, and Pairing Symmetry in Iron-Based Superconductors. Phys. Rev. B, 78:134512, 2008. 
[157] L. Boeri, O. V. Dolgov, and A. A. Golubov. Is $\mathrm{LaFeAsO}_{1-x} \mathrm{~F}_{x}$ an Electron-Phonon Superconductor? Phys. Rev. Lett., 101:026403, 2008.

[158] Q. Si and E. Abrahams. Strong Correlations and Magnetic Frustration in the High $\mathrm{T}_{c}$ Iron Pnictides. Phys. Rev. Lett., 101:076401, 2008.

[159] M. Daghofer, A. Moreo, J. A. Riera, E. Arrigoni, D. J. Scalapino, and E. Dagotto. Model for the Magnetic Order and Pairing Channels in Fe Pnictide Superconductors. Phys. Rev. Lett., 101:237004, 2008.

[160] W.-F. Tsai, Y.-Y. Zhang, C. Fang, and J. Hu. Impurity-Induced Bound States in Iron-Based Superconductors with $s$-Wave $\cos k_{x} \cdot \cos k_{y}$ pairing symmetry. Phys. Rev. B, 80:064513, 2009.

[161] 11, 2009.

[162] T. A. Maier, S. Graser, D. J. Scalapino, and P. J. Hirschfeld. Origin of Gap Anisotropy in Spin Fluctuation Models of the Iron Pnictides. Phys. Rev. B, 79:224510, 2009.

[163] Y.-Y. Zhang, C. Fang, X. Zhou, K. Seo, W.-F. Tsai, B. A. Bernevig, and J. Hu. Quasiparticle Scattering Interference in Superconducting Iron Pnictides. Phys. Rev. B, 80:094528, 2009.

[164] D. Zhang. Nonmagnetic Impurity Resonances as a Signature of SignReversal Pairing in FeAs-Based Superconductors. Phys. Rev. Lett., 103:186402, 2009 .

[165] M. A. Ivanov and Y. G. Pogorelov. Electron Properties of Two-Parameter Long-Range Impurity States. Sov. Phys. JETP, 61:1033, 1985.

[166] E.N. Economou. Green's Functions in Quantum Physics. Springer, Berlin, 2006.

[167] D. V. Efremov, M. M. Korshunov, O. V. Dolgov, A. A. Golubov, and P. J. Hirschfeld. Disorder Induced Transition Between $s_{ \pm}$and $s_{++}$States in Two-Band Superconductors. arXiv:1104.3840 [cond-mat.mtrl-sci], 2011.

[168] M. Tinkham. Introduction to Superconductivity. McGraw Hill, 1995.

[169] N. F. Mott. Electrons in Disordered Structures. Adv. Phys., 16:49, 1967.

[170] I. I. Mazin and J. Schmalian. Pairing Symmetry and Pairing State in Ferropnictides: Theoretical Overview. Physica C, 469:614, 2009.

[171] M. J. Deweert. Proximity-Effect Bilayers with Magnetic Impurities: The Abrikosov-Gor'kov Limit. Phys. Rev. B, 38:732, 1988. 
[172] R. V. A. Srivastava and W. Teizer. Analytical Density of States in the Abrikosov-Gorkov Theory. Solid State Commun., 145:512, 2008.

[173] R. T. Gordon, H. Kim, M. A. Tanatar, R. Prozorov, and V. G. Kogan. London Penetration Depth and Strong Pair Breaking in Iron-Based Superconductors. Phys. Rev. B, 81:180501(R), 2010.

[174] H. Shiba. Classical Spins in Superconductors. Prog. Theor. Phys., 40:435, 1968.

[175] A. I. Rusinov. Superconductivity near a Paramagnetic Impurity. JETP Lett. URSS, 9:85, 1969.

[176] M. Fowler and K. Maki. Conditions for Bound States in a Superconductor with a Magnetic Impurity. Phys. Rev., 164:484, 1967.

[177] R. S. Gonnelli, D. Daghero, G. A. Ummarino, A. Calzolari, M. Tortello, V. A. Stepanov, N. D. Zhigadlo, K. Rogacki, J. Karpinski, F. Bernardini, and S. Massidda. Effect of Magnetic Impurities in a Two-Band Superconductor: A Point-Contact Study of Mn-Substituted $\mathrm{MgB}_{2}$ Single Crystals. Phys. Rev. Lett., 97:037001, 2006.

[178] C. P. Moca, E. Demler, B. Jankó, and G. Zaránd. Spin-Resolved Spectra of Shiba Multiplets from Mn Impurities in $\mathrm{MgB}_{2}$. Phys. Rev. B, $77: 174516,2008$.

[179] S. Onari and H. Kontani. Violation of Anderson's Theorem for the Signreversing $s$-Wave State of Iron-Pnictide Superconductors. Phys. Rev. Lett., 103:177001, 2009.

[180] H. Kontani and S. Onari. Orbital-Fluctuation-Mediated Superconductivity in Iron Pnictides: Analysis of the Five-Orbital Hubbard-Holstein Model. Phys. Rev. Lett., 104:157001, 2010.

[181] Disorder-Induced Transition Between $s_{ \pm}$and $s_{++}$States in Two-Band Superconductors.

[182] Y. Senga and H. Kontani. Impurity Effects in Sign-Reversing Fully Gapped Superconductors: Analysis of FeAs Superconductors. J. Phys. Soc. Jpn, 77:113710, 2008.

[183] T. Kariyado and M. Ogata. Single-Impurity Problem in Iron-Pnictide Superconductors. J. Phys. Soc. Jpn, 79:083704, 2010. 
[184] R. Beaird, I. Vekhter, and J.-X. Zhu. Impurity States in Multiband $s$-Wave Superconductors: Analysis of Iron Pnictides. Phys. Rev. B, $86: 140507,2012$.

[185] S. Kitagawa, Y. Nakai, T. Iye, K. Ishida, Y. F. Guo, Y. G. Shi, K. Yamaura, and E. Takayama-Muromachi. Nonmagnetic Pair-Breaking Effect in $\mathrm{La}\left(\mathrm{Fe} 1-x \mathrm{Zn}_{x}\right) \mathrm{AsO}_{0.85}$ studied by ${ }^{75} \mathrm{As}$ and ${ }^{139} \mathrm{La} \mathrm{NMR}$ and NQR. Phys. Rev. B, 83:180501(R), 2011.

[186] Y. F. Guo, Y. G. Shi, S. Yu, A. A. Belik, Y. Matsushita, M. Tanaka, Y. Katsuya, K. Kobayashi, I. Nowik, I. Felner, V. P. S. Awana, K. Yamaura, and E. Takayama-Muromachi. Large Decrease in the Critical Temperature of Superconducting $\mathrm{LaFeAsO}_{0.85}$ compounds doped with 3\% Atomic Weight of Nonmagnetic Zn Impurities. Phys. Rev. B, 82:054506, 2010.

[187] Y. Li, J. Tong, Q. Tao, C. Feng, G. Cao, W. Chen, F.C. Zhang, and Z.A. Xu. Effect of a Zn Impurity on Tc and its Implications for Pairing Symmetry in LaFeAsO ${ }_{1-x} \mathrm{~F}_{x}$. New J. Phys., 12:083008, 2010.

[188] W. N. Hardy, S. Kamal, D. A. Bonn, K. Zhang, R. Liang, D. C. Morgan, and D. J. Baar. Microwave Surface Impedance of the Cuprate Cuperconductor $\mathrm{YBa}_{2} \mathrm{Cu}_{3} \mathrm{O}_{6.95}$. Physica B, 197:609, 1994.

[189] H. Yamamoto, Z.-Q. Fang, and D.C. Look. Nonalloyed Ohmic Contacts on Low-Temperature Molecular Beam Epitaxial GaAs: Influence of Deep Donor Band. Appl. Phys. Lett., 57:1537, 1990.

[190] V. M. Loktev and Yu. G. Pogorelov. Formation of $d$-Wave Superconducting Order in a Randomly Doped Lattice. Low. Temp. Phys., 27:767, 2001.

[191] R. Kubo. Statistical-Mechanical Theory of Irreversible Processes. I. General Theory and Simple Applications to Magnetic and Conduction Problems. J. Phys. Soc. Jpn., 12:570, 1957.

[192] D.A. Greenwood. The Boltzmann Equation in the Theory of Electrical Conduction in Metals. Proc. Phys. Soc., 71:585, 1958.

[193] Yu. G. Pogorelov and V.M. Loktev. Group Expansions for Impurities in Superconductors. Phys. Rev. B, 69:214508, 2004.

[194] R. J. Elliott, J. A. Krumhansl, and P. L. Leath. The Theory and Properties of Randomly Disordered Crystals and Related Physical Systems. Rev. Mod. Phys., 46:465, 1974. 
[195] Note that this static limit of Eq. (5.13) only defines the conductivity by normal quasiparticles, seen e.g.in normal resistivity by the magnetic flux flow in the mixed state, but otherwise short circuited by the infinite static conductivity due to supercurrents.

[196] Multiband Description of Optical Conductivity in Ferropnictide Superconductors.

[197] M. Abramowitz and I. Stegun. Handbook of Mathematical Functions. Natl. Bureau of Standards, 1964.

[198] V.L. Broude, A.F. Prikhot'ko, and E.I. Rashba. Some Problems of Crystal Luminescence. Sov. Phys. Usp., 2:38, 1959.

[199] Yu. G. Pogorelov and M. C. Santos. Effects of Extended Impurity Perturbation in a $d$-Wave Superconductor. Phys. Rev. B, 71:014516, 2005.

[200] Y. Zhang, Y.-W. Tan, H.L. Störmer, and P. Kim. Experimental Observation of the Quantum Hall Effect and Berry's Phase in Graphene. Nature, 438:201, 2005.

[201] Y. Zhang, T.-T. Tang, C. Girit, Z. Hao, M. C. Martin, A. Zettl, M. F. Crommie, Y.R. Shen, and F. Wang. Direct Observation of a Widely Tunable Bandgap in Bilayer Graphene. Nature, 459:820, 2009.

[202] E.V. Castro, K.S. Novoselov, S.V. Morozov, N.M.R. Peres, J.M.B. Lopes dos Santos, J. Nilsson, F. Guinea, A.K. Geim, and A.H. Castro Neto. Biased Bilayer Graphene: Semiconductor with a Gap Tunable by the Electric Field Effect. Phys. Rev. Lett., 99:216802, 2007.

[203] T. Ando and M. Koshino. Field Effects on Optical Phonons in Bilayer Graphene. J. Phys. Soc. Japan, 78:034709, 2009.

[204] J.D. Bernal. The Structure of Graphite. Proc. R. Soc. Lond. A, 106:749, 1924.

[205] W. Tao, G. Qing, L. Yan, and S. Kuang. A Comparative Investigation of an AB- and AA-Stacked Bilayer Graphene Sheet under an Applied Electric Field: A Density Functional Theory Study. Chinese Phys. B, 21:067301, 2012.

[206] J. Liu and W. Zhang. Bilayer Silicene with an Electrically-Tunable Wide Band Gap. RSC Adv., 3:21943, 2013. 
[207] R.M. Ribeiro and N.M.R. Peres. Stability of Boron Nitride Bilayers: Ground-State Energies, Interlayer Distances, and Tight-Binding Description. Phys. Rev. B, 83:235312, 2011.

[208] J. Slawinska, I. Zasada, and Z. Klusek. Energy Gap Tuning in Graphene on Hexagonal Boron Nitride Bilayer System. Phys. Rev. B, 81:155433, 2010 .

[209] A. Ramasubramaniam, D. Naveh, and E. Towe. Tunable Band Gaps in Bilayer Transition-Metal Dichalcogenides. Phys. Rev. B, 84:205325, 2011.

[210] S.M. Sze. Physics of Semiconductor Devices. 1969.

[211] J. Nilsson and A.H. Castro Neto. Impurities in a Biased Graphene Bilayer. Phys. Rev. Lett., 98:126801, 2007.

[212] A. Feher, E. Syrkin, S. Feodosyev, I. Gospodarev, E. Manzhelii, A. Kotlar, and K. Kravchenko. Nanotechnology and Nanomaterials. New Progress on Graphene Research, chapter 5, page 34. InTech, 2013.

[213] Y. Zhang, A. Mascarenhas, H. P. Xin, and C. W. Tu. Formation of an Impurity Band and its Quantum Confinement in Heavily Doped GaAs:N. Phys. Rev. B., 61:7479, 2000.

[214] E. McCann. Asymmetry Gap in the Electronic Band Structure of Bilayer Graphene. Phys. Rev. B, 74:161403(R), 2006.

[215] V.L. Bonch-Bruevich and S.V. Tyablikov. The Green function method in statistical mechanics. Amsterdam: North-Holland, 1962.

[216] P.T. Araujo, M. Terrones, and M.S. Dresselhaus. Defects and Impurities in Graphene-Like Materials. Mater. Today, 15:98, 2012.

[217] C. Bena. Phys. Rev. Lett., 100:076601, 2008.

[218] J. Nilsson, A. H. Castro Neto, F. Guinea, and N. M. R. Peres. Electronic Properties of Bilayer and Multilayer Graphene. Phys. Rev. B, 78:045405, 2008.

[219] H. P. Dahal, A. V. Balatsky, and J.-X. Zhu. Tuning Impurity States in Bilayer Graphene. Phys. Rev. B, 77:115114, 2008.

[220] D. N. Sheng, L. Sheng, and Z. Y. Weng. Quantum Hall Effect of Dirac Fermions in Graphene: Disorder Effect and Phase Diagram. Phys. Rev. $B, 73: 233406,2006$. 
[221] P. W. Anderson. Localized Magnetic States in Metals. Phys. Rev., 124:41, 1961.

[222] R. R. Biswas, S. Sachdev, and D. T. Son. Coulomb Impurity in Graphene. Phys. Rev. B, 76:205122, 2007.

[223] V. M. Pereira, V. N. Kotov, and A. H. Castro Neto. Supercritical Coulomb Impurities in Gapped Graphene. Phys. Rev. B, 78:085101, 2008.

[224] Y. G. Pogorelov, M. C. Santos, and V. M. Loktev. Impurity Effects on Electronic Transport in Ferropnictide Superconductors. Phys. Rev. B, 88:224518, 2013.

[225] P. Soven. Coherent-Potential Model of Substitutional Disordered Alloys. Phys. Rev. B, 156:809, 1967.

[226] K. Carva, B. Sanyal, J. Fransson, and O. Eriksson. Defect-Controlled Electronic Transport in Single, Bilayer, and N-Doped Graphene: Theory. Phys. Rev. B, 81:245405, 2010.

[227] A. V. Zayats, I. I. Smolyaninov, and A. A. Maradudin. Nano-Optics of Surface Plasmon Polaritons. Phys. Reports, 408:131, 2005.

[228] V. V. Mkhitaryan and E. G. Mishchenko. Localized States due to Expulsion of Resonant Impurity Levels from the Continuum in Bilayer Graphene. Phys. Rev. Lett., 110:086805, 2013.

[229] Z. Ni, Q. Liu, K. Tang, J. Zheng, J. Zhou, R. Qin, Z. Gao, D. Yu, and J. Lu. Tunable Bandgap in Silicene and Germanene. Nano Lett., 12:113, 2012.

[230] Y. G. Pogorelov, M. C. Santos, and V. M. Loktev. Electric Bias Control of Impurity Effects in Bilayer Graphene. Phys. Rev. B, 92:075401, 2015.

[231] G. Fiori and G. Iannaccon. Graphene as a Material for Nanoelectronics. IEEE Electron Device Lett., 30:1096, 2009.

[232] M. Khüne, F. Paolucci, J. Popovic, P. M. Ostrovsky, J. Maier, and J. H. Smet. Ultrafast Lithium Diffusion in Bilayer Graphene. Nature Nanot., 12:895, 2017.

[233] M.-Q. Zhao, Q. Zhang, J.-Q. Huang, G.-L. Tian, J.-Q. Nie, H.-J. Peng, and F. Wei. Unstacked Double-Layer Templated Graphene for High-Rate Lithium-Sulphur Batteries. Nature Comms., 5:3410, 2014. 
[234] H. Yan. Bilayer Graphene: Physics and Application Outlook in Photonics. Nanophotonics, 4:115, 2015.

[235] E. Akbari, R. Yusof, M. T. Ahmadi, A. Enzevaee, M. J. Kiani, H. Karimi, and M. Rahmani. Bilayer Graphene Application on $\mathrm{NO}_{2}$ Sensor Modelling. J. Nanomat., 2014:534105, 2014.

[236] K. S. Novoselov, A. K. Geim, S. V. Morozov, D. Jaing, M. I. Katsnelson, I. V. Grigorieva, S. V. Dubonos, and A. A. Firsov. Two-Dimensional Gas of Massless Dirac Fermions in Graphene. Nature, 438:197, 2005.

[237] A. L. Rakhmanov, F. Nori, A. V. Rozhkov, and A. O. Sboychakov. Electronic Properties of Graphene-Based Bilayer Systems. Phys. Rep., 648:1, 2016.

[238] P. G. Silvestrov and P. Recher. Wigner Crystal Phases in Bilayer Graphene. Phys. Rev. B, 95:075438, 2017.

[239] G. Jaeger. The Ehrenfest Classification of Phase Transitions: Introduction and Evolution. Arch. Hist. Exact. Sci., 53:51, 1998.

[240] E. McCann and M. Koshino. The Electronic Properties of Bilayer Graphene. Rep. Prog. Phys., 76:056503, 2013.

[241] Z. Qiao, W.-K. Tse, H. Jiang, Y. Yao, and Q. Niu. Two-Dimensional Topological Insulator State and Topological Phase Transition in Bilayer Graphene. Phys. Rev. Lett., 107:256801, 2011.

[242] E. Berg, M. S. Rudner, and S. A. Kivelson. Electronic Liquid Crystalline Phases in a Spin-Orbit Coupled Two-Dimensional Electron Gas. Phys. Rev. B, 85:035116, 2012.

[243] J. Jung, M. Polini, and A. H. MacDonald. Persistent Current States in Bilayer Graphene. Phys. Rev. B, 91:155423, 2015.

[244] J.-C. Charlier, J.-P. Michenaud, and X. Gon. First-Principles Study of the Electronic Properties of Simple Hexagonal Graphite. Phys. Rev. B, 46:4531, 1992.

[245] Z. Liu, K. Suenaga, P. J. F. Harris, and S. Iijima. Open and Closed Edges of Graphene Layers. Phys. Rev. Lett., 102:015501, 2009.

[246] A. L. Rakhmanov, A. V. Rozhkov, A. O. Sboychakov, and F. Nori. Instabilities of the AA-Stacked Graphene Bilayer. Phys. Rev. Lett., 109:206801, 2012. 
[247] R. S. Akzyanov, A. O. Sboychakov, A. V. Rozhkov, A. L. Rakhmanov, and F. Nori. AA-Stacked Bilayer Graphene in an Applied Electric Field: Tunable Antiferromagnetism and Coexisting Exciton Order Parameter. Phys. Rev. B, 90:155415, 2014.

[248] G. Kresse and J. Furthmüller. Efficient Iterative Schemes for Ab Initio Total-Energy Calculations using a Plane-Wave Basis Set. Phys. Rev. B, 54:11169, 1996.

[249] G. Kresse and J. Hafner. Ab Initio Molecular Dynamics for Liquid Metals. Phys. Rev. B, 47:R558, 1993.

[250] G. Kresse and J. Furthmüller. Efficiency of Ab-Initio Total Energy Calculations for Metals and Semiconductors using a Plane-Wave Basis Set. Comput. Mat. Sci., 6:15, 1996.

[251] G. Kresse and D. Joubert. From Ultrasoft Pseudopotentials to the Projector Augmented-Wave Method. Phys. Rev. B, 59:1758, 1999.

[252] L. He, F. Liu, G. Hautier, M. J. T. Oliveira, M. A. L. Marques, F. D. Vila, J. J. Rehr, G.-M. Rignanese, and A. Zhou. Accuracy of Generalized Gradient Approximation Functionals for Density-Functional p Perturbation Theory Calculations. Phys. Rev. B, 89:064305, 2014.

[253] F. Favot and A. Dal Corso. Phonon Dispersions: Performance of the Generalized Gradient Approximation. Phys. Rev. B, 69:11427, 1999.

[254] E. Lora da Silva, Jonathan M. Skelton, Stephen C. Parker, and Aron Walsh. Phase Stability and Transformations in the Halide Perovskite $\mathrm{CsSnI}_{3}$. Phys. Rev. B, 91:144107, 2015.

[255] H. J. Monkhorst and J. D. Pack. Special Points for Brillouin-Zone Integrations. Phys. Rev. B, 13:5188, 1976.

[256] Estelina Lora da Silva. Modelling Structural Phase Transitions. PhD thesis, University of Bath, 2018.

[257] A. Togo and I. Tanaka. First Principles Phonon Calculations in Materials Science. Scr. Mater., 108:1, 2015.

[258] A. Togo, F. Oba, and I. Tanaka. First-Principles Calculations of the Ferroelastic Transition between Rutile-Type and $\mathrm{CaCl}_{2}-\mathrm{Type} \mathrm{SiO}_{2}$ at High pPressures. Phys. Rev. B, 78:134106, 2008.

[259] P. Y. Yu and M. Cardona. Fundamentals of Semiconductors: Physics and Materials Properties. Number 104. 1996. 
[260] M. Gajdoš, K. Hummer, G. Kresse, J. Furthmüller, and F. Bechstedt. Linear Optical Properties in the PAW Methodology. Phys. Rev. $B, 73: 045112,2006$.

[261] Y. Xu, X. Li, and J. Dong. Infrared and Raman Spectra of AA-Stacking Bilayer Graphene. Nanotechnology, 21:065711, 2010.

[262] M. Hanfland, H. Beister, and K. Syassen. Graphite under Pressure: Equation of State and First-Order Raman Modes. Phys. Rev. B, 39:12598, 1989.

[263] J.-K. Lee, S.-C. Lee, J.-P. Ahn, S.-C. Kim, J. I. B. Wilson, and P. John. The Growth of AA Graphite on (111) Diamond. J. Chem. Phys., 129:234709, 2008.

[264] B. Skinner, B. I. Shklovskii, and M. B. Voloshin. Bound State Energy of a Coulomb Impurity in Gapped Bilayer Graphene. Phys. Rev. B, 89:041405(R), 2014.

[265] M. Mohr, J. Maultzsch, E. Dobardžić, S. Reich, I. Miložević, M. Damnjanović, A. Bosak an M. Krisch, and C. Thomsen. Phonon Dispersion of Graphite by Inelastic X-Ray Scattering. Phys. Rev. B, 76:035439, 2007.

[266] A. Cocemasov and D. Nik. Phonons in Bilayer Graphene. http://tkea. com.ua/siet/archive/2013-t2/130.pdf, 2008.

[267] S. Li and Y. Chen. Thermal Transport and Anharmonic Phonons in Strained Monolayer Hexagonal Boron Nitride. Scient. Rep., 7:43956, 2017.

[268] J.-W. Jiang, J.-S. Wang B.-S. Wang, and H. S. Park. A Review on the Flexural Mode of Graphene: Lattice Dynamics, Thermal Conduction, Thermal Expansion, Elasticity and Nanomechanical Resonance. J. Phys.: Condens. Matter, 27:083001, 2015.

[269] A. I. Cocemasov, D. L. Nika, and A. A. Balandin. Phonons in Twisted Bilayer Graphene. Phys. Rev. B, 88:035428, 2013. 
